\title{
New Pyrazole- and Triazine-Based Ligands as Scaffolds for Oligonuclear Complexes: From Multiredox Stability to Heteronuclear Single Molecule Magnets
}

\author{
Dissertation \\ zur Erlangung des Doktorgrades \\ der Mathematisch-Naturwissenschaftlichen Fakultäten \\ der Georg-August-Universität zu Göttingen
}

vorgelegt von

Diplom-Chemiker

Animesh Das

aus Bakcha (Indien)

Göttingen 2009 
D7

Referent: Prof. Dr. Franc Meyer

Korreferent: Prof. Dr. Dietmar Stalke

Tag der mündlichen Prüfung: 20.01.2010 


\section{Table of Contents}

\section{Introduction}

Motivation and Concepts 3

\section{Introduction to Magnetochemistry}

2.1. Basic Terms and Definitions of Magnetic Properties................................8

2.2. Single Molecule Magnets (SMMs) ..................................... 11

2.3. Synthetic Strategies for Making Polynuclear Complexes ............................ 14

2.4. Thesis Overview...................................................... 16

3. Triazine-Based Compartmental Ligands and their Binuclear $\mathrm{Cu}(\mathrm{II})$, Tetranuclear Mn(III), and Fe(III) Complexes: Syntheses, Structures, Magnetic Properties and Spectro-Electrochemistry

3.1. Introduction............................................................. 17

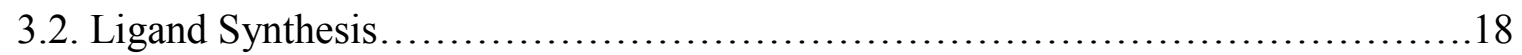

3.3. Synthesis and Structural Characterisation of Metal Complexes....................22

3.4. Spectroscopy in Solution................................................ 28

3.5. Spectroscopy in the Solid states......................................... 37

3.6. Conclusions ........................................................ 42

4. Triazine Based Mononuclear, Dinuclear, and 1-D Chain Complexes of Manganese (II) with Some Auxiliary ligands: Synthesis, Structures, and Magnetic Properties

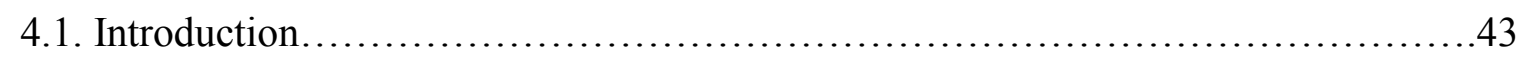

4.2. Synthesis and Structural Characterisation of Metal Complexes....................44

4.3. Magnetic Properties............................................................49

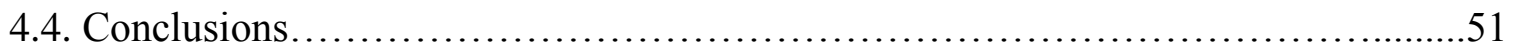

5. Pyrazole Based Homometallic Complexes: $\mathrm{M}_{4} \mathrm{O}_{4}\left(\mathrm{M}=\mathrm{Ni}^{\mathrm{II}}\right.$, $\mathrm{Co}^{\mathrm{II}}$, $\mathrm{Fe}^{\text {II }}$ ) Cubes with High-Spin Ground States

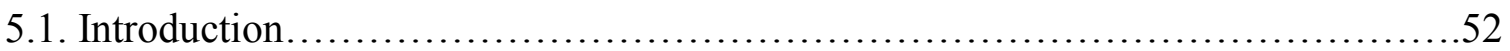

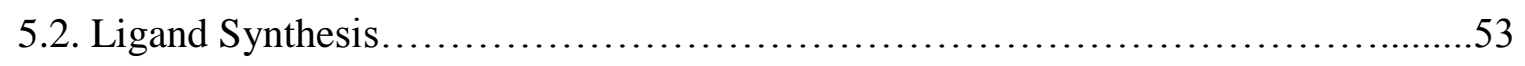

5.3. Cluster Synthesis and Structural Characterisation...............................55 


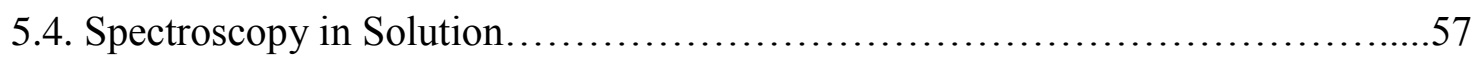

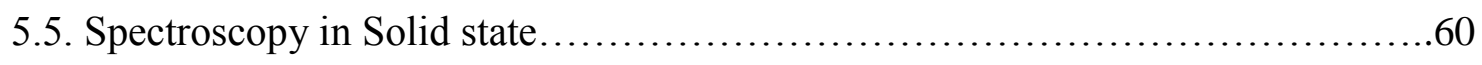

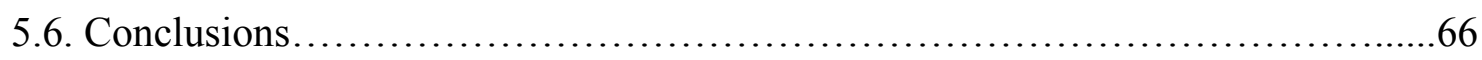

\section{A New Family of Heterometal Single Molecule Magnets: Observation of Quantum Tunnelling Steps in the Hysteresis Loops of $\left\{\mathrm{Mn}_{2} \mathrm{Ni}_{3}\right\}$ Clusters}

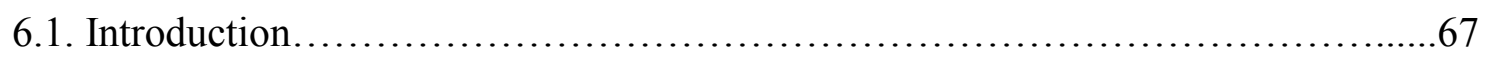

6.2. Synthesis and Structural Characterisation of Metal Complexes.....................68

6.3. Magnetic Properties.............................................................. 76

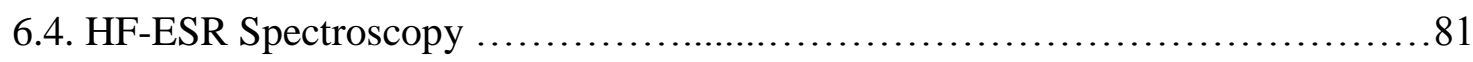

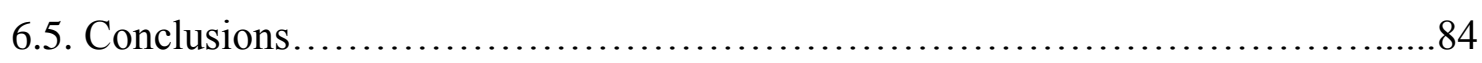

\section{High Nuclearity Aggregate of Mixed Valent Manganese Clusters and Heteronuclear $\mathrm{Mn}^{\mathrm{III}}{ }_{2} \mathrm{Ln}^{\mathrm{III}}{ }_{2}$ SMMs: Synthesis, Structural Characterization, and Magnetic Properties}

7.1. Introduction .85

7.2. Synthesis, Structure and Magnetic Properties of Mixed valent

Manganese clusters .86

7.3. Synthesis, Structure and Magnetic Behavior of heteronuclear

$\left[\mathrm{NHEt}_{3}\right]\left[\left(\mathrm{L}^{12}\right)_{2} \mathrm{Mn}_{2}{ }_{2}^{\mathrm{III}}\left\{\left(\mathrm{NO}_{3}\right)_{2} \mathrm{Ln}^{\mathrm{III}}\right\}_{2}\left(\mathrm{O}_{2} \mathrm{CPh}\right)_{3}\left(\mu_{4}-\mathrm{O}\right)\right] \cdot \mathrm{x} \mathrm{CH}_{3} \mathrm{CN} \cdot \mathrm{y} \mathrm{CH} \mathrm{CH}_{2} \mathrm{Cl}_{2} \ldots \ldots . . .94$

7.4. Conclusions

\section{New Binucleating Pyrazolate Ligands with Phenol Side arms- Potential Scaffolds for Bimetallic Multiradical Chemistry}

8.1. Introduction ............................................................. 102

8.2. Ligand Synthesis....................................................... 104

8.3. Synthesis and Molecular Structure of Metal Complexes.......................... 105

8.4. Magnetic Properties of Copper(II) Complexes..................................... 109

8.5. Spectroscopy in Solution................................................. 111

8.6. Conclusions....................................................... 122

\section{Experimental Section}

9.1. General Considerations. 
9.2. Synthesis of Ligands and Ligand Precursors

9.3. Synthesis of Metal Complexes and Cluster Compounds

10. Crystallography. 140

Bibliography. 150

Appendix A. List of Free Ligands. 163

Appendix B. List of Crystalline Compounds. 165

Appendix C. List of Chemical Terms

Appendix D. Overview of Magnetic Parameters 168

Appendix E. Overview of Electrochemical Measurements .169

Appendix F. Overview of Mössbauer Measurements .169

Abbreviation .170 


\section{Chapter 1}

\section{Introduction}

Magnetism is a topic which is highly underrated although it has a profound impact on our day to day life it is much less researched on in academia. It all started with the ancient Greeks, originally those near the city of Magnesia, and also the early Chinese who knew about strange and rare stones, possibly chunks of iron ore struck by lightning, with the power to attract iron in a magical way. A steel needle stroked with such a "lodestone" became "magnetic" as well, and the Chinese found that such a needle, when freely suspended, pointed north-south. This led to the discovery and subsequent exploitation of magnets and magnetism, and human civilization has tremendously benefited from it ever since. Magnets are so essential and ubiquitous to a plethora of devices in our daily life that sometimes they are taken for granted. Be it the simple speakers or headphones, or the complicated motors or telecommunication devices, magnets find an application almost everywhere. Modern day magnetic materials include magnetic alloys and oxides, particularly ferrites such as $\mathrm{MgFe}_{2} \mathrm{O}_{4}$, which can function in transformer cores, magnetic recording or information storage devices. The global market for magnetic materials is valued at $\$ 50$ billion and has a projected growth rate of $10 \%$ annually. Additionally, with the advent of GNR (genetics, nanotechnology and robotics) this burgeoning field of magnetism and magnetic materials will undoubtedly expand as the $21^{\text {st }}$ century progresses, since magnets will be crucial to the development of the so called "smart materials" and "smart systems." The behavior of any magnetic material is essentially dependent on the presence of unpaired electrons, or more precisely the spin associated with the unpaired electrons. The magnetic field associated with a magnetic substance is the result of an electrical charge in motion, specifically the spin and orbital angular momenta of electrons within atoms of a material. These materials are so called atom-based magnets which mean that their active spins are located in the atomic orbitals of the constituent metal ions. ${ }^{1}$ They often have transition or lanthanide metal ions with unpaired spins, which provide the magnetic moment, and organic groups to mediate magnetic interactions between these metal centres. This has produced magnetic materials of various dimensions, and some with longrange magnetic ordering. ${ }^{2}$ They not only have the potential use as functional materials, but also exhibit fascinating structural diversity. As a result, there has been an increasing interest in designing 1D, 2D and 3D magnetic systems which can provide a better understanding of magneto-structural relationships. ${ }^{3}$ These systems have been found to exhibit diverse magnetic 
properties. ${ }^{4}$ The molecular approach offers a unique opportunity because the properties of the precursors and magnetic bridges can be chosen beforehand in order to obtain magnets with desired physical properties.

An exciting development was the discovery of single molecule magnets (SMMs), ${ }^{5}$ which are magnetic molecular clusters. These molecules exhibit slow relaxation of magnetisation at low temperatures, and thus represent a molecular approach to nanomagnets. Their special characteristics arise from purely molecular properties. These molecules should possess a spin ground state $(S)$, where $S \geq 1 / 2$, and a uniaxial magnetoanisotropy, where $D<0$. The combination of these properties can lead to an energy barrier to the thermal relaxation of the magnetisation. The magnetic bistability arising from this energy barrier indicates potential applications for these materials in information storage devices, whereas a single molecule can act as the smallest possible unit of magnetic memory. They have many important advantages over conventional nanoscale magnetic particles composed of metals, metal alloys or metal oxides because of their uniform size, solubility in organic solvents and readily alterable peripheral ligands. In order to obtain new SMMs, polynuclear metal compounds which contain interacting metal centres held together by bridging units, such as oxygen atoms derived from oxides, hydroxide, alkoxide and carboxylates have been synthesised. Much effort has been applied in the design and synthesis of such compounds and in the investigation of their magnetic properties. Extended networks of magnetically coupled SMMs can lead to new magnetic behaviour induced by the intrinsic properties of the magnetic units, such as the high-spin state, the Ising-type anisotropy and quantum effects.

In this research, the goal was to synthesize and study a range of compounds starting from discrete molecules to extended solids. A variety of magnetic properties, such as SMM behaviour and long-range magnetic ordering, was expected from these compounds. In this way, a comprehensive study on different kinds of magnetic behaviour in different systems can be achieved. To synthesise such compounds, a commonly used strategy is to employ appropriate bridging ligands that can bind several metal centres and transmit magnetic interactions. In this context, triazine based compartmental ligands and tridentates diol ligands with a central pyrazole unit were used as scaffolds with different metal ions under various reaction conditions. It has been observed that heterometallic complexes can have interesting magnetic properties; therefore, more than one type of metal ion was used to obtain compounds containing two different transition metal ions $(3 d-3 d)$ or of transition and lanthanide metal ions $(3 d-4 f)$. The effect of ligand modifications on the structure and properties of the complexes was also investigated. 


\section{Motivation and Concepts}

The development of molecule-based magnets requires the specific alteration of magnetic properties by established organic or coordination chemistry techniques and the combination of magnetic properties with other mechanical, electrical and/or optical properties in harmony with simplicity of fabrication. ${ }^{6}$ The rational design of polynuclear complexes with high spin ground states between the paramagnetic centers is central to the development of new classes of molecule-based magnets. Different strategies (e.g, double exchange, spin polarization and orthogonality of magnetic orbitals) are to be used in order to prepare high-spin molecules in a single cluster, in which one of the well known strategies is spin polarisation mechanism. This mechanism arises from the molecular orbital model proposed by Longuet-Higgis for conjugated alternate hydrocarbons, ${ }^{7}$ which results in ferromagnetic or antiferromagnetic coupling between two radicals depending on the nature of the bridge. When the radicals are separated by a meta-phenylene bridge they are ferromagnetically coupled because two unpaired electrons reside in a pair of mutually orthogonal but degenerate SOMOs.

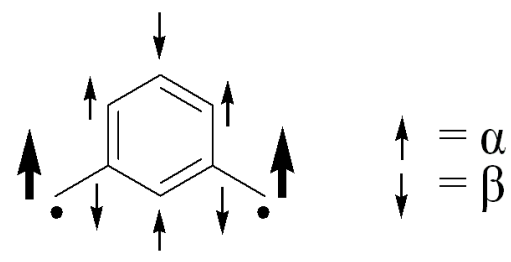

Scheme 1.1. meta-Phenylene based linkage with $\alpha$ and $\beta$ spin distribution.

A consequence of this molecular orbital behavior is the alternation of $\alpha$ and $\beta$ spin density induced on the bridging atoms, which has actually been detected and measured in some cases. This simple principle has been behind the preparation of numerous polyradicals exhibiting a very high spin ground state. Though this mechanism is well known for organic polyradicals systems, it has received limited attention in the case of metal coordinated systems. We can apply this knowledge in our choice of ligands (Scheme 1.1). Initially this concept has been applied to binuclear transition metal complexes bridged in meta-phenylene arrangement using a variety of bridging ligands, and in some cases it exhibits ferromagnetic interactions between the metal ions. ${ }^{8}$ However, many complexes with the same kinds of bridging ligands exhibit antiferromagnetic interactions between the transition metal ions. ${ }^{9}$ The orientation of the magnetic orbital on each metal site relative to the plane of the bridging benzene ring was 
found to be crucial for a ferromagnetic or an antiferromagnetic contribution of the super exchange interaction as well as of the spin-polarization mechanism.

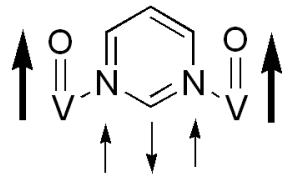

A

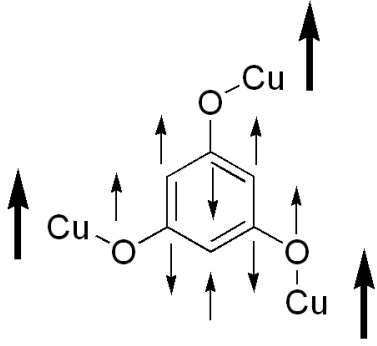

B

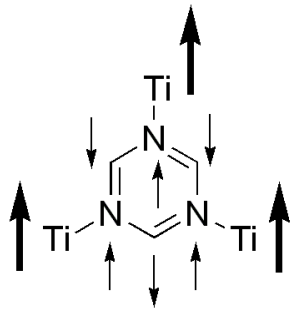

C

\section{Scheme 1.2.}

Based on spin-polarisation concept, some well-known example of different meta-phenylene bridged transition metal complexes with ferromagnetically coupled systems are shown in Scheme 1.2. It displays that pyrimidine is the ferromagnetic exchange coupler in dinuclear oxovanadium complex (Scheme 1.2.A), ${ }^{10}$ and the application of modified 1,3,5trihydroxybenzene (phloroglucinol) ligands, or 1,3,5-trithiocyanurate ligands also led to the ferromagnetic exchange coupler in trinuclear $\mathrm{Cu}(\mathrm{II})$ (Scheme 1.2.B), ${ }^{11}$ or $\mathrm{Ti}(\mathrm{III})$ complexes (Scheme 1.2.C) ${ }^{12}$ respectively. In this context, we wanted to investigate whether modified 1,3,5-triazine-ligands may be used for the synthesis of trinuclear transition metal complexes with ferromagnetic interactions between the paramagnetic centers (other than $\mathrm{Ti}^{\mathrm{III}}$ centre) based on the spin-polarization mechanism. Thus, our first aim of this research was to synthesize trinuclear transition metal complexes involving triazine- $\mathrm{N}$ in metal ion coordination, and to get a high spin ground state through spin polarisation mechanism, that may lead to the development of new types of molecule-based magnets. And these threedirectional trimetallic units could be a valuable precursor for the assembly of 2D sheets with inherent threefold symmetry, such as $2 \mathrm{D}$ honeycomb networks. ${ }^{13}$ It is noteworthy that there are only few structurally characterized complexes known to date in which all triazine- $\mathrm{N}$ are involved in metal coordination, ${ }^{14}$ and very few of them feature paramagnetic metal centers. Hence, designing of appropriate ligand is the key to obtain the paramagnetic trimetallic complex involving triazine- $\mathrm{N}$ in metal ion binding site. Varieties of compartmental ligands are sketched in this work (Scheme 1.3), those are listed below: 
<smiles>O=C(NNc1nc(NNC(=O)c2ccccn2)nc(NNC(=O)c2ccccn2)n1)c1ccccn1</smiles><smiles>Oc1cccnc1-c1nc(-c2ncccc2O)nc(-c2ncccc2O)n1</smiles>

$\mathrm{H}_{3} \mathrm{~L}^{2}$<smiles>[R]N(/N=C/c1ccccc1O)c1nc(N([R])/N=C/c2ccccc2O)nc(N([R])/N=C/c2ccccc2O)n1</smiles>

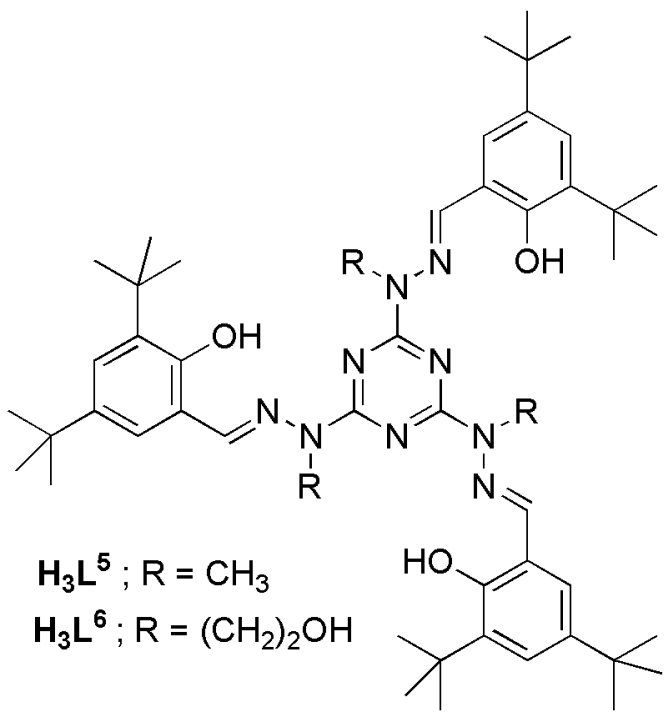<smiles>CN(/N=C/c1ccccn1)c1nc(N(C)/N=C/c2ccccn2)nc(N(C)/N=C/c2ccccn2)n1</smiles>

\section{Scheme 1.3.}

In principle triazine based trimetallic complex can formed in two ways, either in strategies I, or in strategies II (Scheme 1.4), for first case, we designed the ligand $\mathbf{H}_{3} \mathbf{L}^{\mathbf{1}}, \mathbf{H}_{3} \mathbf{L}^{\mathbf{3}}, \mathbf{H}_{\mathbf{3}} \mathbf{L}^{\mathbf{5}}$, and $\mathbf{L}^{7}$ and for second case, we designed the ligand $\mathbf{H}_{3} \mathbf{L}^{2}, \mathbf{H}_{3} \mathbf{L}^{4}$, and $\mathbf{H}_{3} \mathbf{L}^{6}$. In the first case, complex can be stabilised by strong $\sigma$-donor site of the the ligand such as amide (in $\mathbf{H}_{3} \mathbf{L}^{\mathbf{1}}$ ), or 
hydroxyl group (in $\mathbf{H}_{\mathbf{3}} \mathbf{L}^{\mathbf{3}}$ and $\mathbf{H}_{\mathbf{3}} \mathbf{L}^{\mathbf{5}}$ ) and in later complex can stabilise through five and six membered chelation by hydroxyl group and strong $\mathrm{N}$-donor pyridine (in $\mathbf{H}_{3} \mathbf{L}^{2}$ ) or six and seven membered chelation by hydroxyl groups (in $\mathbf{H}_{3} \mathbf{L}^{4}$, and $\mathbf{H}_{3} \mathbf{L}^{6}$ ). Chapter 3 and 4 report the synthesis and spectroscopic characterisation of all compartmental ligands, and its relevant metal complexes.

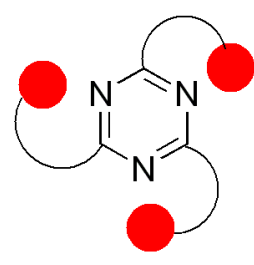

I

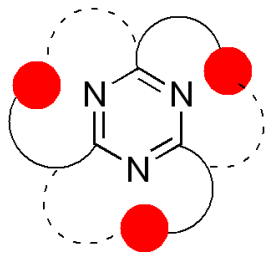

II

Scheme 1.4.

Another aim of this research work was to design and synthesis of new molecule based materials and characterise their magnetic behaviour. In order to construct the polnynuclear complexes, the concept of self-assembly of the paramagnetic metal ions with suitable pyrazole containing ligand was applied. In principal pyrazoles are very useful functional bridging ligands since they allow the connection of two metal ions by a variable superexchange pathway. ${ }^{15}$ Substitutions in the heterocyclic ring can render novel ligands, thus providing a variety of coordination compounds. ${ }^{16}$ In recent years, 3(5)-methyl-5(3)-(2hydroxyphenyl)pyrazole $\left(\mathbf{H}_{2} \mathbf{L}^{\mathbf{8}}\right)$ is very useful and versatile ligand, which exhibits a rich coordination chemistry. ${ }^{17}$ But from magnetic point of view most of the cluster is showing intramolecular antiferromagnetic interaction between the metal ions ${ }^{17 a, b, c}$ and gives rise to zero spin ground state, and in very few cases show ferromagnetic interaction. ${ }^{17 \mathrm{~d} \text {, e }}$ Thus, the choice of new ligands is prerequisite for new molecule based magnets. So we modified the previously reported ligand $\mathbf{H}_{2} \mathbf{L}^{8}$ and synthesized a new pyrazole based diol ligand $\mathbf{H}_{2} \mathbf{L}^{9}$ (Scheme 1.5) which can be regarded as an attractive scaffold for assembling multinuclear complexes because

(i) it may act as a tridentate ligand with sufficient flexibility in one arm (the hydroxyethyl subunit); (ii) and it provides, after deprotonation, two distinct potential bridging units (phenoxy and alkoxy). 
<smiles>Cc1cc(-c2ccccc2O)n[nH]1</smiles>

$\mathrm{H}_{2} \mathrm{~L}^{8}$<smiles>OCCn1nc(-c2ccccc2O)cc1-c1ccccc1</smiles>

$\mathrm{H}_{2} \mathrm{~L}^{10}$<smiles>OCCn1ccc(-c2ccccc2O)n1</smiles>

$\mathrm{H}_{2} \mathrm{~L}^{9}$<smiles>Oc1ccccc1-c1ccn(CC(O)c2ccccc2)n1</smiles>

$(R)-H_{2} L^{11}$

\section{Scheme 1.5.}

In this work, initial attempts were made to explore polynuclear metal complexes with high spin ground state and high magnetic anisotropy using self-assembly of $3 d$ transition metal ions (mainly, $\mathrm{Mn}^{\mathrm{II}}, \mathrm{Fe}^{\mathrm{II}}, \mathrm{Co}^{\mathrm{II}}$, and $\mathrm{Ni}^{\mathrm{II}}$ ) with those ligands (Scheme 1.5) and despite the advanced synthetic methods and magnetic properties of polynuclear complexes of the $d$-block homo metals, less attention has been devoted to the synthesis and magnetic properties of mixed metal ions, even though SMMs will lead to larger magnetic anisotropy, higher ground spin states and a better understanding of quantum tunneling effects through synergy of heterometal spins. Therefore, our research was focused on new SMMs with mixed anisotropic paramagnetic metal ions, mainly high spin $\mathrm{Mn}(\mathrm{III})$ with other $3 d$ or $4 f$ metal ions. The strategy is obviously to take advantage of the $\mathrm{Mn}^{\mathrm{III}}$ ion's significant spin, and/or its large single-ion zero-field splitting, as reflected in a large magnetic anisotropy $(D)$ value, to generate "better SMMs" distinctly different from the homometallic ones. Thus construction of new heterometal SMMs by the development of new reaction systems with those ligands is another area of research interest. 


\section{Chapter 2}

\section{Introduction to Magnetochemistry}

\subsection{Basic terms and definitions of magnetic properties}

Magnetochemistry is the study of the interrelationship between the structure of a compound and its magnetic properties. Magnetic measurements can give information about the electronic structure and magnetic exchange interactions in metal complexes. This brief introduction defines and explains basic magnetic properties. For additional details, standard text books are available. $^{18}$

Behavior of a substance in magnetic field: When a substance is placed in a magnetic field $(H)$, the density of lines of force in the sample, known as the magnetic induction or magnetic flux density $(B)$ is related to $(H)$ by the permeability of the substance $(\mu)$ :

$$
\begin{aligned}
& B=\mu H \\
& B=\mu_{0} H+\mu_{0} M
\end{aligned}
$$

where $\mu_{0}$ is the permeability of free space and $M$ is the magnetization or magnetic moment of the sample. Here, $\mu_{0} H$ is the induction generated by the field alone and $\mu_{0} M$ is the additional induction contributed by the sample. The magnetic susceptibility is defined as the ratio of magnetization to the field.

$$
\chi=M / H
$$

and therefore,

$$
\mu=\mu_{0}(1+\chi)
$$

The ratio $\mu / \mu_{0}$, which equals to $(1+\chi)$, is known as the relative permeability, $\mu_{\mathrm{r}}$. The magnetic behaviour of all materials can be categorized by the values of $\chi$ and $\mu_{\mathrm{r}}$ and by their temperature dependence and field dependence. For a vaccum, $\mu_{\mathrm{r}}=1$. When $\chi$ is negative, the $\mu_{\mathrm{r}}<1$ and the substance are diamagnetic. Such a substance allows a smaller number of lines of force to pass through it (as compared to that in vacuo), and thus it prefers to move to the region of the lowest magnetic field strength. On the other hand, when $\chi$ is positive, then $\mu_{\mathrm{r}}>1$ 
and the substance is paramagnetic. Such a substance allows more lines of force to pass through it, and therefore prefers to move to the region of the highest magnetic field.

Effects of temperature-Curie and Curie-Weiss Laws: The susceptibilities of different kinds of magnetic materials are distinguished by both their temperature dependence and their absolute magnitudes. Many paramagnetic substances obey the simple Curie law, especially at high temperature. This states that the magnetic susceptibility is inversely proportional to temperature:

$$
\chi=C / T
$$

Where $C$ is the Curie constant. The Curie law is obeyed in materials where not any spontaneous interaction between adjacent unpaired electrons. When there are some spontaneous interactions between adjacent spins, a better fit to the high-temperature, paramagnetic behaviour is often provided by the Curie-Weiss law:

$$
\chi=C /(T-\theta)
$$

where $\theta$ is the Weiss constant. Depending on the ferro- or antiferromagnetism, $\theta$ is positive or negative, respectively. These two types of behavior are shown in Figure 2.1.
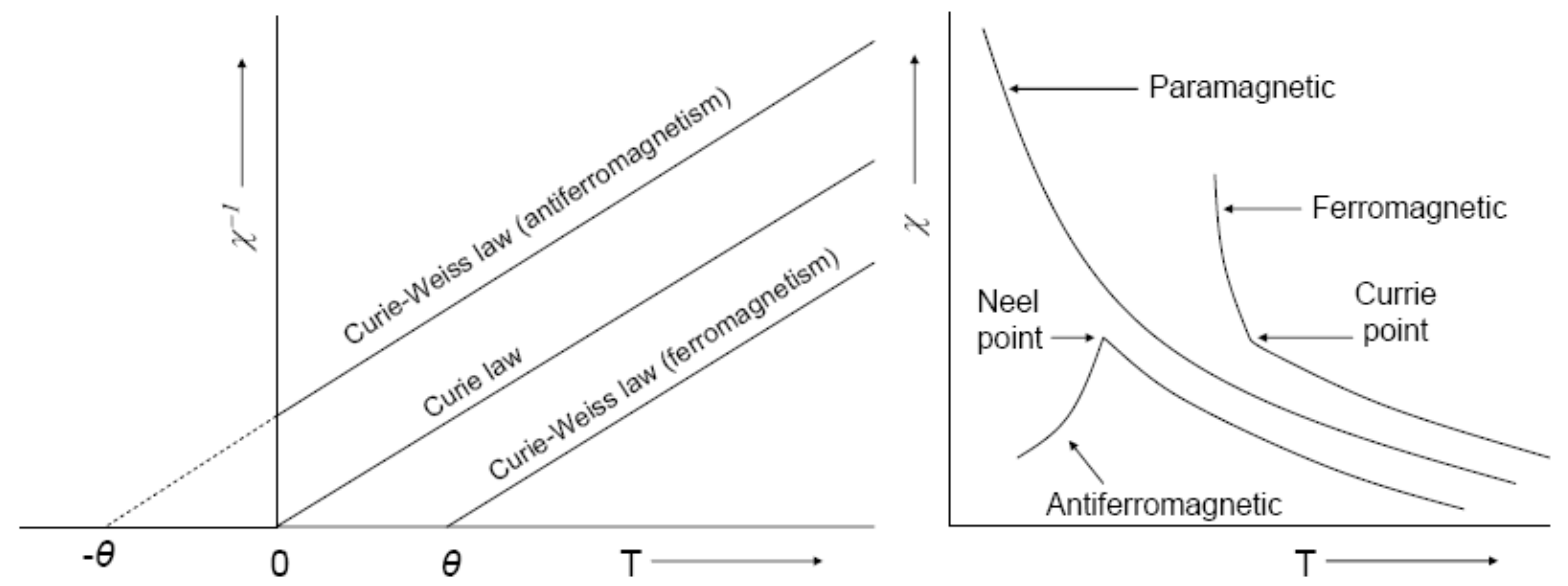

Figure 2.1. $\chi^{-1}$ versus $T$ plot and $\chi$ versus $T$ plot for the ferromagnetic, antiferromagnetic and paramagnetic materials.

Diamagnetic substances have their magnetic susceptibility invariant to temperature, whereas the magnetic susceptibility of a paramagnet follows an inverse relationship with temperature. 
An antiferromagnet has a characteristic Néel temperature $\left(T_{N}\right)$ above which it behaves like a paramagnet, but below this temperature, its magnetic susceptibility decreases with decreasing temperature (Figure 2.1, right). A ferromagnet has a characteristic Curie temperature $\left(T_{c}\right)$ above which it behaves like a paramagnet, but below this temperature, its magnetic susceptibility increases rapidly.

Magnetic hysteresis: When a ferromagnetic material is magnetized in one direction, it will not relax back to zero magnetization when the imposed magnetizing field is removed. It must be driven back to zero by a field in the opposite direction. When the magnetization is plotted, a loop called a hysteresis loop results (Figure 2.2). The lack of retraceability of the magnetization-curve is the property called hysteresis, and it is related to the existence of magnetic domains in the material. Once the magnetic domains are reoriented, it takes some energy to turn them back again. This property of ferromagnetic materials is useful as a magnetic "memory". When the ferromagnetic material retains an imposed magnetization indefinitely, it is termed as a "permanent magnet". The magnetic memory aspects of iron and chromium oxides make them useful in audio tape recording and for the magnetic storage of data on computer disks. As shown in Figure 2.2, the phenomena can be described in the following way: Starting at zero field, the material follows a non-linear magnetization curve and reaches the saturation level, when all the spins are aligned along the direction of a field (Figure 2.2, point a).

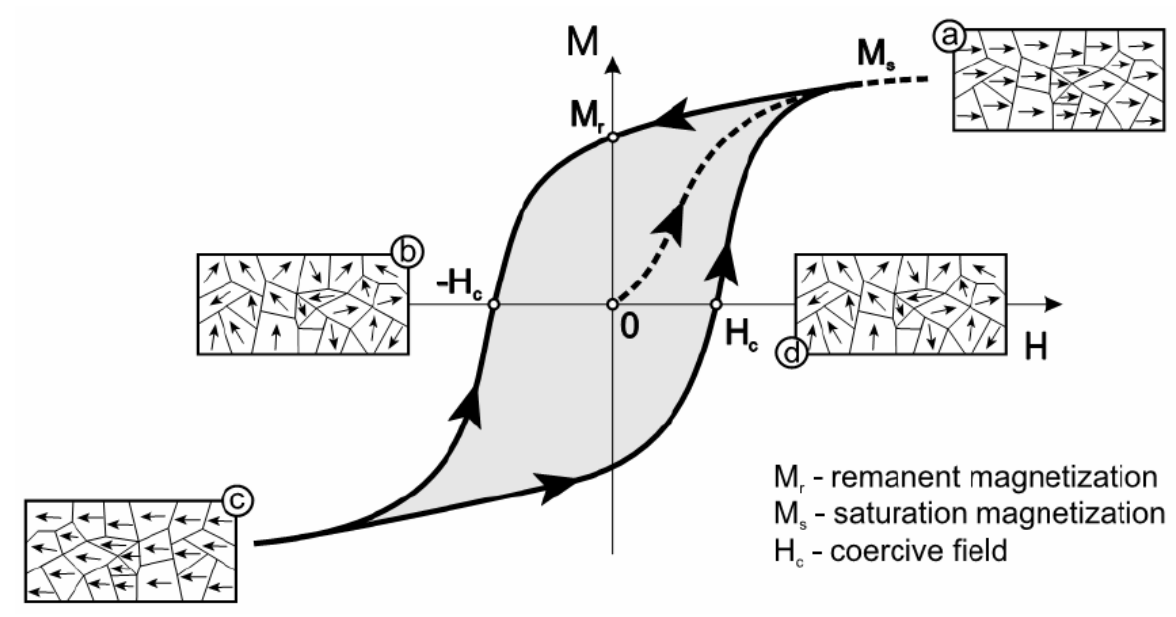

Figure 2.2. The plot of magnetization $(M)$ versus magnetic field $(H)$ for a ferromagnetic compound is illustrated by showing the orientations of the electrons in each domain at different points of the loop.

As the field is decreased and drops to zero, the ferromagnetic material retains a considerable degree of magnetization $\left(M_{\mathrm{r}}\right)$. It can be thought of as "remembering" the previous state of 
magnetization. At this point, when $H=0$, a ferromagnet is not fully demagnetized and only a partial domain reorientation has happened. When a reverse field is applied, at a given point the magnetization reaches zero $\left(-H_{\mathrm{c}}\right)$. This field required to demagnetize a ferromagnetic material is called the "coercive field" $\left(H_{\mathrm{c}}\right.$, point b). Saturation of magnetization also achieved by applying an opposite field (point c) and then decreasing the field and reversing it again in the opposite direction completes the loop after going through a point with zero magnetization (point d).

\subsection{Single Molecule Magnets (SMMs)}

A single molecule can show all the above mentioned properties of a bulk magnet. These single molecules, termed as single-molecule magnets (SMMs) were discovered during the course of investigations into the miniaturization of the size of magnetic devices. Many difficulties have been encountered in the "top-down" approach. For example, these are often nano particles with inhomogeneous size distributions of the domains. Decreasing the domainsize also makes the magnet less efficient. As a result, research is now intensely focused on the "bottom-up" approach in order to produce nano-scale materials starting from the atomic or molecular level. ${ }^{19}$ The ability of a single molecule to behave like a bulk magnet caught the attention of researchers because such systems may lead to the smallest possible magnetic devices. ${ }^{20}$ These SMMs are of interest because they exhibit magnetic bistability of pure molecular origin. The slow relaxation in the magnetization is the source of this interesting behaviour and is due to the presence of an energy barrier which has to be overcome in the reversal of the magnetic moment. This behaviour has been compared to that of super paramagnetic materials. Super paramagnetic behaviour occurs when a single magnetically ordered domain has a reorientation barrier that is comparable to the thermal energy. ${ }^{21}$ As a consequence, the magnetization flips freely and its time average is zero, as in a paramagnet. In an external field, it behaves like a paramagnet until the temperature is sufficiently reduced such that the barrier can not be easily overcome. Thus the magnetization is blocked, below a blocking temperature, $T_{B}$, and the super paramagnet reverts to its usual bulk behaviour (i.e. ferro-, ferri-, or antiferromagnetic). For a molecule to have a barrier in a system of ground spin $S$, it must have the magnetic sub-states, $M= \pm S$ lying lowest in energy. In the case of axial symmetry, where the system has an integer spin in the ground state, the height of the energy barrier $(\Delta E)$ can be associated with the difference between the lowest energy $M= \pm S$ states and the highest energy $M=0$ state. For a negative axial zero-field splitting parameter, $D, \Delta E=|D| S^{2}$ (for integer-spin) and $\Delta E=|D|\left(S^{2}-1 / 4\right)$ (for integer-spin). To produce new 
nanomagnetic materials based on molecular clusters, it is therefore necessary to achieve a reasonably large ground state spin. For this purpose $S=5 / 2$ ions such as high-spin $\mathrm{Mn}^{\mathrm{II}}$ and $\mathrm{Fe}^{\mathrm{III}}$ are well-suited metal ions for SMM. Subsequently a large ground state spin can be achieved just by using a small number of ions.

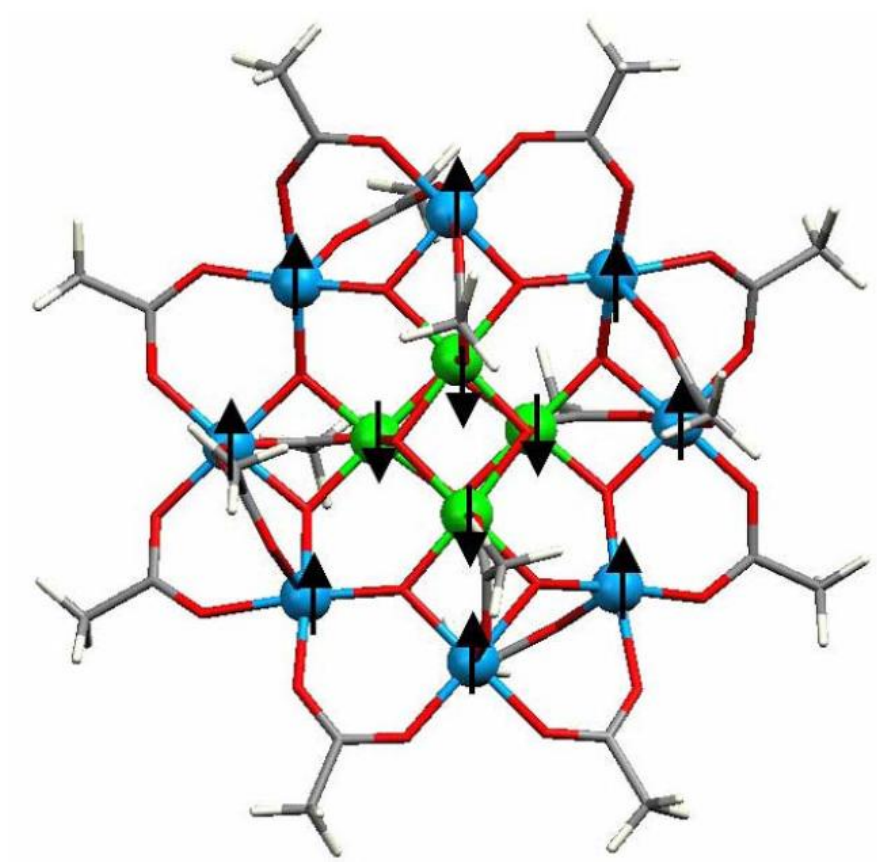

Figure 2.3. Structure of $\left[\mathrm{Mn}_{12} \mathrm{O}_{12}\left(\mathrm{CH}_{3} \mathrm{COO}\right)_{16}\left(\mathrm{H}_{2} \mathrm{O}\right)_{4}\right] \cdot 2 \mathrm{CH}_{3} \mathrm{COOH} \cdot 4 \mathrm{H}_{2} \mathrm{O}$ (abbreviated $\mathrm{Mn}_{12}$-ac). ${ }^{5}$ Green, blue, red and grey centres represent $\mathrm{Mn}^{\mathrm{IV}}, \mathrm{Mn}^{\mathrm{III}}, \mathrm{O}$ and $\mathrm{C}$ atoms, respectively. The arrows indicate the resultant spin orientation of different Mn ions. Four $\mathrm{Mn}^{4+}\left(3 d^{3}, S=3 / 2\right)$ are ferromagnetically coupled for a total spin of $S=6$. The remaining eight $\mathrm{Mn}^{3+}\left(3 d^{4}, S=2\right)$ are also ferromagnetically coupled to one another in a crown structure resulting into a total spin of $S=16$. The $\mathrm{Mn}^{3+}$ crown is antiferromagnetically coupled to the inner $\mathrm{Mn}^{4+}$ cubane, yielding a total spin of $S=10$

The prototypical SMM $\left[\mathrm{Mn}_{12} \mathrm{O}_{12}\left(\mathrm{CH}_{3} \mathrm{COO}\right)_{16}\left(\mathrm{H}_{2} \mathrm{O}\right)_{4}\right] \cdot 2 \mathrm{CH}_{3} \mathrm{COOH} \cdot 4 \mathrm{H}_{2} \mathrm{O},{ }^{5,}, 22$ abbreviated to $\mathrm{Mn}_{12}$-ac, is a suitable example to illustrate $\mathrm{SMM}$ behaviour. The structure of the compound is shown in (Figure 2.3). The $\mathrm{Mn}_{12}$-ac cluster contains twelve manganese ions, bridged via oxygen atoms. In the structure, four $\mathrm{Mn}^{\mathrm{IV}}$ ions reside on the alternating vertices of a central cubane. All of these $\mathrm{Mn}^{4+}\left(3 d^{3}, S=3 / 2\right)$ ions are ferromagnetically coupled. The other vertices are occupied by four $\mu_{4}$-oxo groups. The other eight ions are $\mathrm{Mn}^{3+}$, and they define a crown-like geometry surrounding the $\mathrm{Mn}_{4}$-cubane. All these eight $\mathrm{Mn}^{3+}\left(3 d^{4}, S=2\right)$ ions are also ferromagnetically coupled. Although the $\mathrm{Mn}^{4+}$ and $\mathrm{Mn}^{3+}$ ions in the inner cubane and outer crown are ferromagnetically coupled among themselves, the total spins of these two parts are coupled antiferromagnetically to give a total net spin of $10(8 \times 2-4 \times 3 / 2=10)$. 

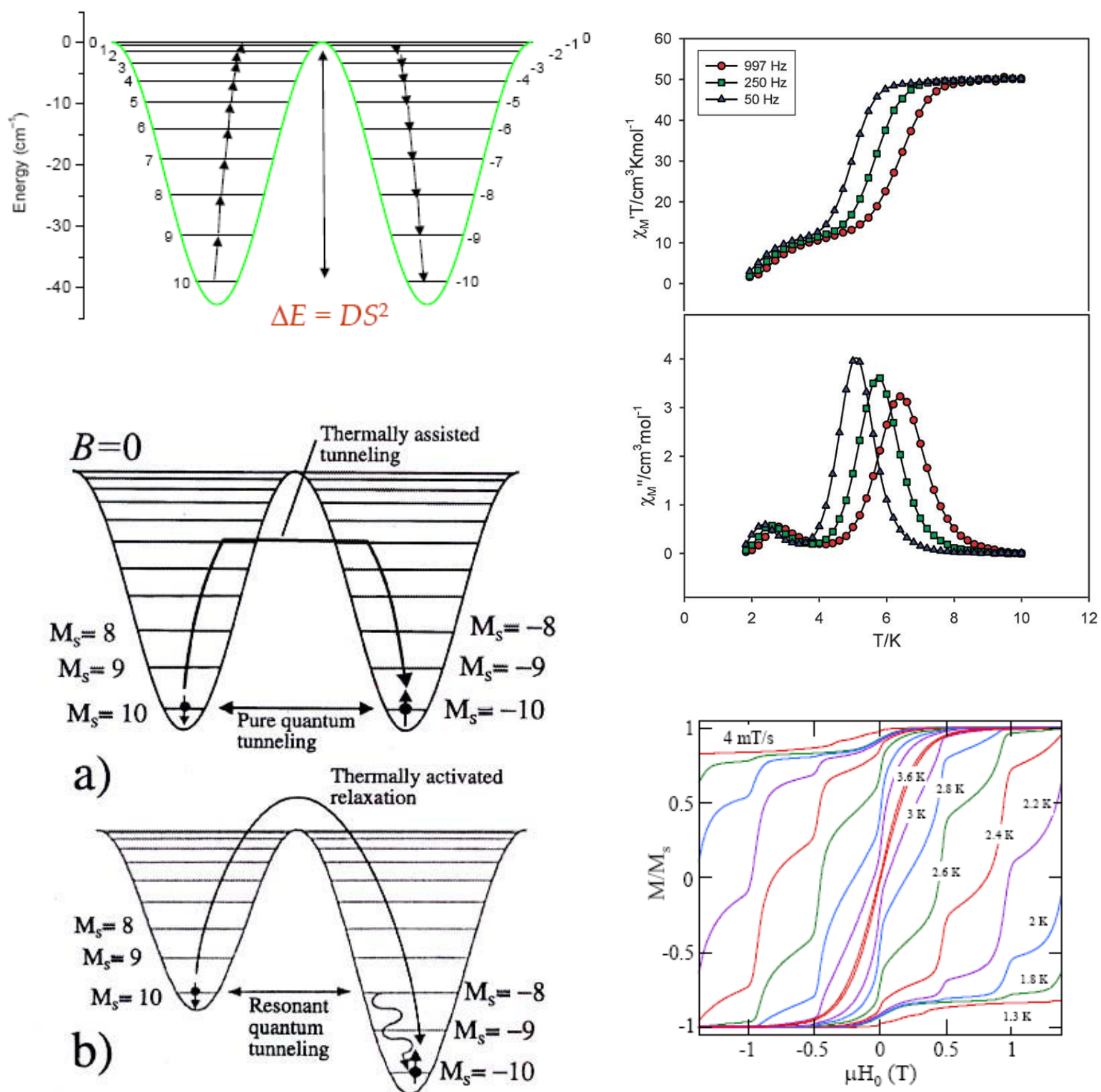

Figure 2.4. Energy level diagram of $S=10$, and the horizontal lines are the energies of the spin microstates belonging to the $S=10$ multiplet (top-left), and possible tunneling mechanisms (left-bottom) for a $\mathrm{Mn}_{12}$ complex. In-phase $\chi_{\mathrm{M}}{ }^{\prime} T$, and out-of-phase $\chi_{\mathrm{M}}{ }^{\prime \prime}$, ac susceptibility signals (top-right), and typical hysteresis loops (bottom-right) with steps, for a $\mathrm{Mn}_{12} \mathrm{SMM}$.

To reverse the spin of the molecule from along the -10 (spin up) to the +10 (spin down) axis of the molecule, a potential energy barrier, $U=S^{2}|D| \approx 50 \mathrm{~cm}^{-1}$ (as $S=10, D=-0.5 \mathrm{~cm}^{-1}$ ) for $\mathrm{Mn}_{12} \mathrm{ac}$, must be overcome (Figure 2.4; top-left). For this reason, SMMs exhibit slow magnetization relaxation at low temperatures. Experimental evidence for this behavior is supported by the appearance of concomitant frequency-dependent in-phase $\left(\chi_{M}^{\prime}\right)$ and out-ofphase $\left(\chi_{\mathrm{M}}\right.$ ") signals in alternating current (ac) magnetic susceptibility measurements, as shown 
in Figure 2.4 (top-right). Frequency-dependent ac signals are necessary but not sufficient proof that an SMM has been obtained. The observance of hysteresis loops in magnetization versus direct current $(\mathrm{dc})$ field scans, with coercivities increasing with decreasing temperature and increasing scan rates, is an unambiguous confirmation that an SMM has been obtained. ${ }^{23}$ Sometimes the hysteresis loops have steps (Figure 2.4; bottom-right), which are due to quantum tunneling, and each step corresponds to a sudden increase in the magnetization relaxation rate. The QTM can be of different kinds (Figure 2.4; bottom-left) and these will be discussed later (chapter 6).

From a magnetic point of view, SMMs are preferred over classical nanoscale magnetic particles because they have a single, well defined ground state spin $S$ which is a true quantum spin system, and highly ordered assemblies of SMMs can be obtained in crystalline form. At the same time, from a synthetic chemist's point of view, these SMMs can be easily synthesized at room temperature by solution methods and we can obtain a collection of truly mono disperse particles of nanoscale dimensions which have true solubility (rather than colloid formation) in organic solvents. Additionally, the central core containing the metals is engulfed in a protective shell of organic groups which can be easily varied by ligand substitution, thus providing advantages for certain applications. Due to the strong need for SMMs with even larger $S$ values and more negative $D$ values, numerous synthetic strategies aimed at the improvement of these materials have been considered. Several new strategies and SMMs will be discussed in the ensuing chapters.

\subsection{Synthetic strategies for making polynuclear complexes}

Several synthetic routes are being pursued to construct new magnetic materials that, ideally, satisfy the criteria for SMMs. Two well-known approaches have been developed in the literature. The first one can be termed "rational molecular design". In this approach, the components of the reaction system display lower flexibility, such that the geometry and properties of the products can often be forecasted. Some very interesting systems have been created in this manner, taking advantage of the linearity of the cyanide ligand in combination with the predictable geometry and electronic structure of specific transition metals. ${ }^{24}$ An alternative "rational" approach, the synthesis of complicated polytopic and polynucleating ligands, has allowed the preparation of many magnetically interesting clusters with fascinating pre-determined geometries. ${ }^{25}$

The second approach is based on the serendipitous self-assembly of flexible ligands with metal ions. ${ }^{26}$ A prerequisite for successful application of self-assembly is to accept the 
impossibility of understanding the influence of all factors involved in a reaction on the resulting product. Therefore a wide range of conditions for any specific reaction is explored. The substitution pattern of the ligand or ligands, the metal salt, the metal: ligand ratio, the crystallisation solvent, the solution concentration and the crystallisation temperature may all play a role in the formation of a complex. Although this synthetic approach produces unpredictable results, minor variations in the ligands may influence the structure and solubility. The use of mixtures of organic ligands can form complexes of higher nuclearities. $^{26 a-c}$ Different coligands having carboxylate or hydroxyl groups can also be fruitful to construct large polynuclear clusters. Varying the coligand by increasing the number of donor groups might lead to the incorporation of more metal centres. When alcohol-based solvents (methanol or water) were employed, hydrolysis reactions of the solvents can be expected, forming hydroxy or oxo ligands. One further possible variation is to add bridging species like acetate or azide, which can act as additional linkers between the metal ions, or can fill any empty coordination sites on the metal centres.

Heterometallic complexes can be obtained using two different types of metal ions. The presence of two types of metal centres can give rise to interesting magnetic properties and can allow further investigation of the exchange interaction between two different metal ions. ${ }^{26 \mathrm{~d}-\mathrm{f}}$ This was pursued using two different transition metals or a combination of transition and lanthanide metal ions. The lanthanide ions behave as hard acids prefering oxygen (O-) rather than nitrogen $(\mathrm{N}-)$ donors, while d-block metal ions are borderline acids, having a tendency to coordinate to $\mathrm{N}$-donors as well as $\mathrm{O}$ - donors. Consequently, a typical approach in constructing $3 d-4 f$ complexes is by self-assembly of different metal ions with ligands containing both Oand $\mathrm{N}$-donors. Thus $\mathrm{O}-$ and $\mathrm{N}$ - donor ligands are may be suitable for the synthesis of mixedmetal clusters.

The transition metal ions used in the following chapters were $\mathrm{Mn}(\mathrm{II}), \mathrm{Fe}(\mathrm{II}), \mathrm{Co}(\mathrm{II}), \mathrm{Ni}(\mathrm{II})$ and $\mathrm{Fe}(\mathrm{III})$. With O- and N- donors, Mn(II) has five unpaired electrons in its high-spin state which can give large ground state spins on aggregates. It can be easily oxidized to $\mathrm{Mn}$ (III), which is anisotropic in octahedral geometry, and can give rise to high zero-field splitting values $(D)$ leading to a significant barrier to magnetisation reversal $(U)$. As a result, mixed valence manganese compounds are likely to have a combination of both high spin and high anisotropy. $\mathrm{Cu}$ (II) ions have one unpaired electron, making the interpretation of magnetic interactions relatively straightforward. $\mathrm{Fe}(\mathrm{III}), \mathrm{Fe}(\mathrm{II}), \mathrm{Co}(\mathrm{II})$, and $\mathrm{Ni}(\mathrm{II})$ have also significant number of unpaired electrons in their high-spin state, and they can also have a zero-field splitting (depending on the envirnement) which can be useful for synthesizing SMMs. 
Lanthanides (Ln) have the advantages that they can have a large number of unpaired $f$ electrons and considerable single-ion anisotropy. The origin of SMM behaviour in lanthanide containing compounds is, however, more complicated than that of d-block transition metal ions since there is likely to be a significant orbital component. The effect of spin-orbit coupling increases as the atomic number increases, with the exception of the $4 f^{7}$ configuration, which has no first-order angular momentum. As a result, it is useful to synthesise $\mathrm{Gd}$ analogues of $\mathrm{Ln}$ complexes in order to interpret the magnetic properties. Recently, most of the research has been directed solely towards lanthanide clusters but not hybrid lanthanide-transitionmetal clusters. ${ }^{27}$ The use of lanthanides to modulate the magnetic properties of transition-metal single-molecule magnets has become faciniated research area, mainly as a result of the magnetic anisotropy of some lanthanides that can increase the blocking temperature for reversal of magnetization.

\subsection{Thesis overview}

The thesis is organized in the following ways. Chapter 3 reports the synthesis, and characterisation of new 1,3,5 triazine-based compartmental ligands, and its multiredox metal complexes of $\mathrm{Cu}$ (II), and $\mathrm{Mn}$ (III) whose relevance span the magnetic materials as well as the bioinorganic research areas. Chapter 4 describes the synthesis and magnetic properties of new mononuclear, dinuclear and 1-D coordination polymer of $\mathrm{Mn}(\mathrm{II})$ with sodium azide and sodium dicyanamide as auxiliary ligands. Chapter 5 depicts the synthesis, and spectroscopic characterisation of new pyrazole-derived ligand scaffolds, and its tetranuclear alkoxo bridged cube shaped complexes with high spin ground states of $\mathrm{Fe}(\mathrm{II})$ and $\mathrm{Ni}(\mathrm{II})$. Chapter 6 is concerned with the new family of 1-D exchange biased $\mathrm{Mn}^{\mathrm{III}}{ }_{2} \mathrm{Ni}_{3}{ }_{3}$-heterometal SMMs, where the exchange bias can be controlled by chemical and structural modifications. Chapter 7 consists of the use of pyrazole based diol ligand to synthesize the magnetically interesting new mixed valent $\mathrm{Mn}(\mathrm{II} / \mathrm{III})$ clusters and a new family of $\mathrm{Mn}_{2} \mathrm{Ln}_{2}$-heterometal SMMs. Chapter 8 presents the synthesis of a new pyrazolate-based bridging ligand with two phenol side arms $\left(\mathbf{H}_{3} \mathbf{L}^{\mathbf{1 2}}\right)$, and the study of its binuclear $\left(\mathrm{Cu}^{2+}\right.$ and $\left.\mathrm{Ni}^{2+}\right)$ and tetranuclear $\left(\mathrm{Zn}^{2+}\right)$ complexes in metal-radical chemistry. 


\section{Chapter 3}

\section{Triazine-Based Compartmental Ligands and their Binuclear $\mathrm{Cu}(\mathrm{II})$, Tetranuclear $\mathrm{Mn}(\mathrm{III})$, and $\mathrm{Fe}(\mathrm{III})$ Complexes: Syntheses, Structures, Magnetic Properties and Spectro-Electrochemistry}

\subsection{Introduction}

1,3,5-Triazine (s-triazine) derivatives are of considerable current interest in coordination chemistry and supramolecular chemistry. ${ }^{28,}{ }^{29}$ Among the many reasons for this are: (i) the electron-deficient s-triazine ring has shown the ability to exhibit unsual anion- $\pi$-contacts ${ }^{30}$ and intricate $\mathrm{H}$-bonding networks; ${ }^{31}$ (ii) s-triazine is a rigid three-directional $C_{3}$-symmetric tecton and its derivatives may serve as versatile ligands towards transition metals; ${ }^{32}$ and (iii) the meta-phenylene disposition of the triazine- $\mathrm{N}$ atoms may give rise to ferromagnetic interactions via the spin-polarization mechanism in cases where several metal ions are bound to the triazine ring. ${ }^{14 \mathrm{f}}$ With regard to the latter two aspects, however, the electron-deficient 1,3,5-triazine has proven quite reluctant to bind to three metal ions. As a result there are only few structurally characterized complexes known to date in which all triazine-N are involved in metal coordination, ${ }^{14}$ and very few of them feature paramagnetic metal centers. Furthermore, s-triazine appears to be quite susceptible to hydrolytic ring cleavage upon metal activation. ${ }^{33}$ Hence the potential of the s-triazine ring as a magnetic coupler in the field of molecular magnetism is hardly exploited yet.

A further interesting aspect is the redox properties of s-triazine, which makes 2,4,6trisubstituted triazines important building blocks in electronic and polymeric materials. ${ }^{34}$ This may also lead to new facets in triazine coordination chemistry in cases where the triazine behaves as a non-innocent ligand. In the last two decades it has been increasingly realized that oxidations of transition metal complexes can be either metal- or ligand-centered. ${ }^{35}$ In view of the biological relevance of tyrosl radicals, much work in this field has focused on the redox properties of metal phenolate complexes. ${ }^{36}$ In this contribution new triazine based ligands (Scheme 1.3) with different substituents in 1,3,5 positions were prepared. Syntheses and spectroscopic characterisation of some relevant metal complexes using those lignads are described in detail in this chapter. 


\subsection{Ligand Synthesis}

All the triazine-based compartmental ligands were synthesized by the reaction with cyanuric chloride and a nucleophile, in where cyanuric chloride as the sole starting material. Compound $\mathbf{H}_{3} \mathbf{L}^{\mathbf{1}}$ was obtained in one step process as outlined in Scheme 3.1. Reaction of 6 equiv. of pyridine-2-carboxylic acid hydrazide ${ }^{37}$ with 1 equiv. of cyanuric chloride in dry acetonitrile under reflux for $6 \mathrm{~h}$, leds to the desired product in $30 \%$ yield. It was characterized by NMR spectroscopy, EI mass spectrometry and micro analysis.<smiles>Clc1nc(Cl)nc(Cl)n1</smiles><smiles>NNC(=O)c1ccccn1</smiles><smiles>C#N</smiles><smiles>O=C(NNc1nc(NNC(=O)c2ccccn2)nc(NNC(=O)c2ccccn2)n1)c1ccccn1</smiles>

Scheme 3.1. Synthesis of ligands $\mathbf{H}_{3} \mathbf{L}^{\mathbf{1}}$.

The compound $\mathbf{H}_{3} \mathbf{L}^{2}$ was obtained in multistep process as outlined in Scheme 3.2, where 3hydroxo-2-bromo pyridine is used as precursor. It was transformed into the corresponding trimethylsilyl derivative of 2-Bromo-3-(trimethylsilyloxy)pyridine ${ }^{38}$ by treating with hexamethyldisilazane. To this $n$-BuLi was added in THF at low temperature, followed by drop wise addition of $1 / 3$ equiv. of cyanuric chloride to afford triazine containing trisubstituted trimethylsilyl derivative as a red solid. Finally trimethylsilyl group was deprotected by treating with dry methanol and led to the desired product 2,4,6-Tris(3hydroxy-2-pyridyl)-1,3,5-triazine in $11 \%$ yield. It was confirmed by NMR spectroscopy, EI mass spectrometry and micro analysis. 
$\underset{B 0^{\circ} \mathrm{C}}{\stackrel{\mathrm{NH}\left(\mathrm{SiMe}_{3}\right)_{2}}{\longrightarrow}}$

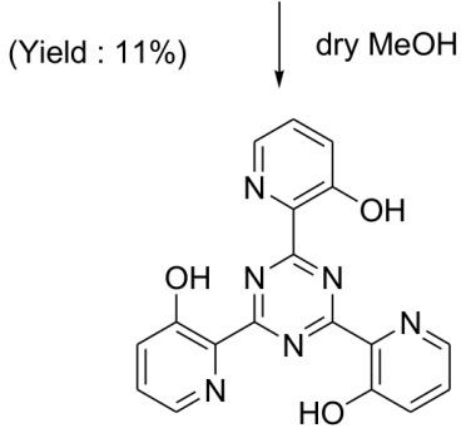

$\left(\mathrm{H}_{3} \mathrm{~L}^{2}\right)$

Scheme 3.2. Synthesis of ligand $\mathbf{H}_{3} \mathbf{L}^{2}$.

The schiff-base type of ligands $\mathbf{H}_{3} \mathbf{L}^{3}, \mathbf{H}_{6} \mathbf{L}^{4}, \mathbf{H}_{3} \mathbf{L}^{5}, \mathbf{H}_{6} \mathbf{L}^{6}$, and $\mathbf{L}^{7}$ have been made in two step process in where the inetrermediates $1 \& 2$ (Scheme 3.3) are obtained upon reaction of cyanuric chloride with the respective hydrazine derivative (e.g, methyl hydrazine or 2hydroxyethyl hydrazine). Addition of the hydrazine is best carried out at $0^{\circ} \mathrm{C}$, the reaction mixture then warmed to room temperature and finally heated to reflux for $3 \mathrm{~h}$. and then condensation of 3 equivalents of corresponding aldehyde with 1 equivalent of intermediates 1 or 2 in methanol gave a desired liganda as a white powders in $25-94 \%$ yield, in which $\mathbf{H}_{3} \mathbf{L}^{5}$ is well soluble in organic solvents like $\mathrm{CHCl}_{3}, \mathrm{CH}_{2} \mathrm{Cl}_{2}, \mathrm{Et}_{2} \mathrm{O}$, EtOAc, hot THF, PhCl, and DMSO. However solubility of $\mathbf{H}_{3} \mathbf{L}^{3}, \mathbf{H}_{3} \mathbf{L}^{4}$, and $\mathbf{L}^{7}$ are only in hot DMF and DMSO, in contrast $\mathbf{H}_{6} \mathbf{L}^{6}$ is sparingly soluble only in $\mathrm{CHCl}_{3}$, and hot DMSO. All the ligands have been characterized by NMR spectroscopy, EI mass spectrometry and micro analysis (Chapter 9.2). To further confirm the integrity of the triazine core, single crystals of intermediate $\mathbf{1}$ (as the hydrochloride salt) and $\mathbf{H}_{3} \mathbf{L}^{\mathbf{5}}$ were grown from $\mathrm{MeCN}$ and chlorobenzene, respectively, and characterised by X-ray diffractometry. Compound $\mathbf{1}$ crystallizes in the monoclinic space group $P 2_{1} / c$, while $\mathbf{H}_{3} \mathbf{L}^{\mathbf{5}}$ crystallizes in the monoclinic space group $P 2_{1} / n$ with two molecules and one chlorobenzene in the asymmetric unit. Molecular structures of $\mathbf{1}$ and $\mathbf{H}_{\mathbf{3}} \mathbf{L}^{\mathbf{5}}$ are depicted in Figure 3.1. 


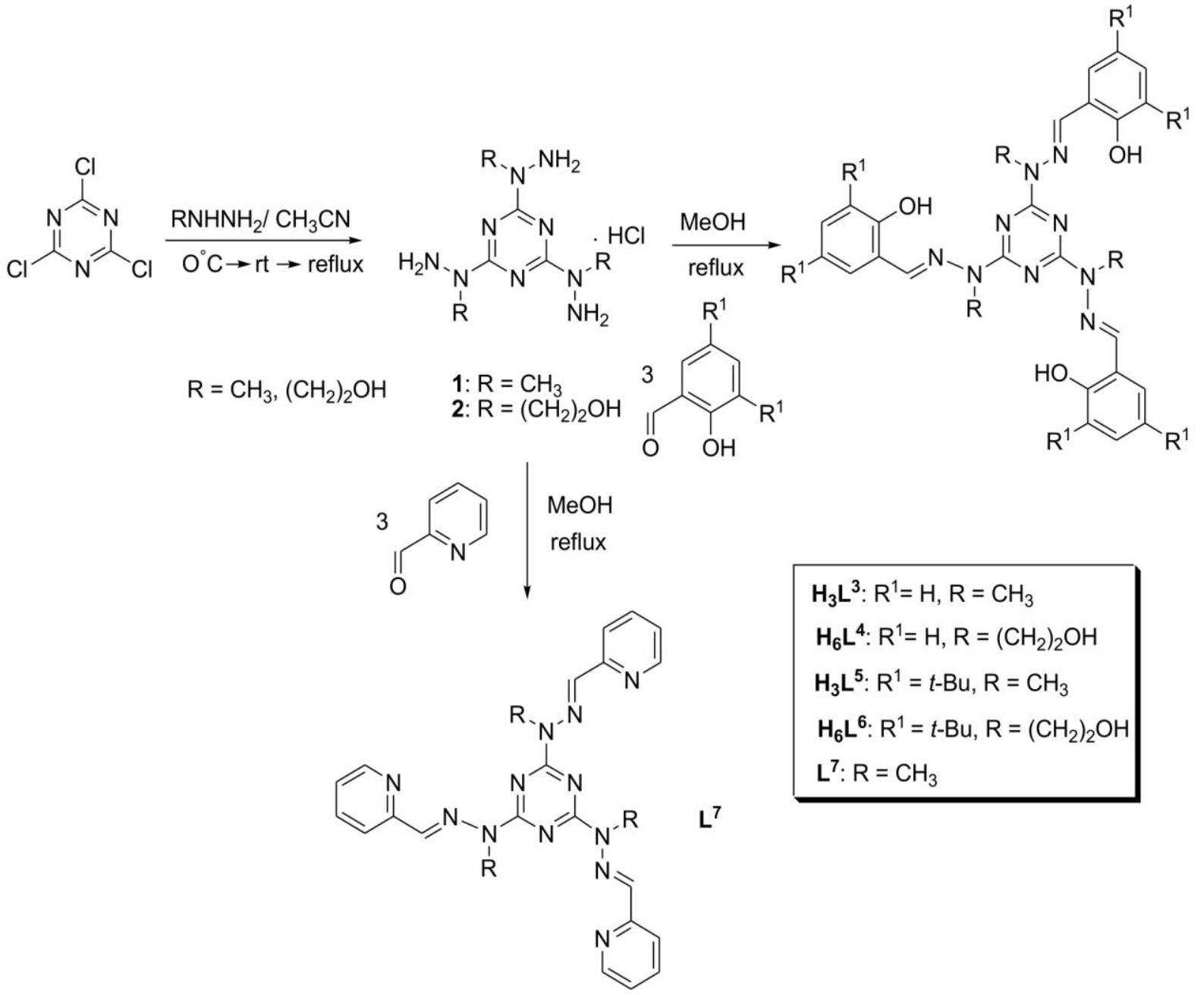

Scheme 3.3. Synthesis of 1,3,5 substituted triazine ligand $\mathbf{H}_{3} \mathbf{L}^{3}, \mathbf{H}_{6} \mathbf{L}^{4}, \mathbf{H}_{3} \mathbf{L}^{5}, \mathbf{H}_{6} \mathbf{L}^{6}$ and $\mathbf{L}^{7}$.

The amine and triazine hydrogen atoms of the cation $\mathbf{1}$ and the chlorine anion are part of a network of manifold intermolecular and intramolecular hydrogen bonding interactions which are depicted in Figure 3.1 and Figure 3.2. In $\mathbf{H}_{3} \mathbf{L}^{\mathbf{5}}$ three phenolic hydrogens present are involved in intramolecular hydrogen bonding to the respective imino nitrogens leading to the formation of a six-membered ring (N-C-C-C-O-H). The metric parameters involved in this interaction, viz., $\mathrm{H} \cdots \mathrm{N}\left(\operatorname{av} .1 .81 \AA\right.$ ) and the $\mathrm{O}-\mathrm{H} \cdots \mathrm{N}$ bond angles (av 146.1 ${ }^{\circ}$ ), are in keeping with the hydrogen bonding trends observed in such types of interacting units. ${ }^{39}$ Stacking or close contacts between ring atoms are found in both compounds which were not shown here. 

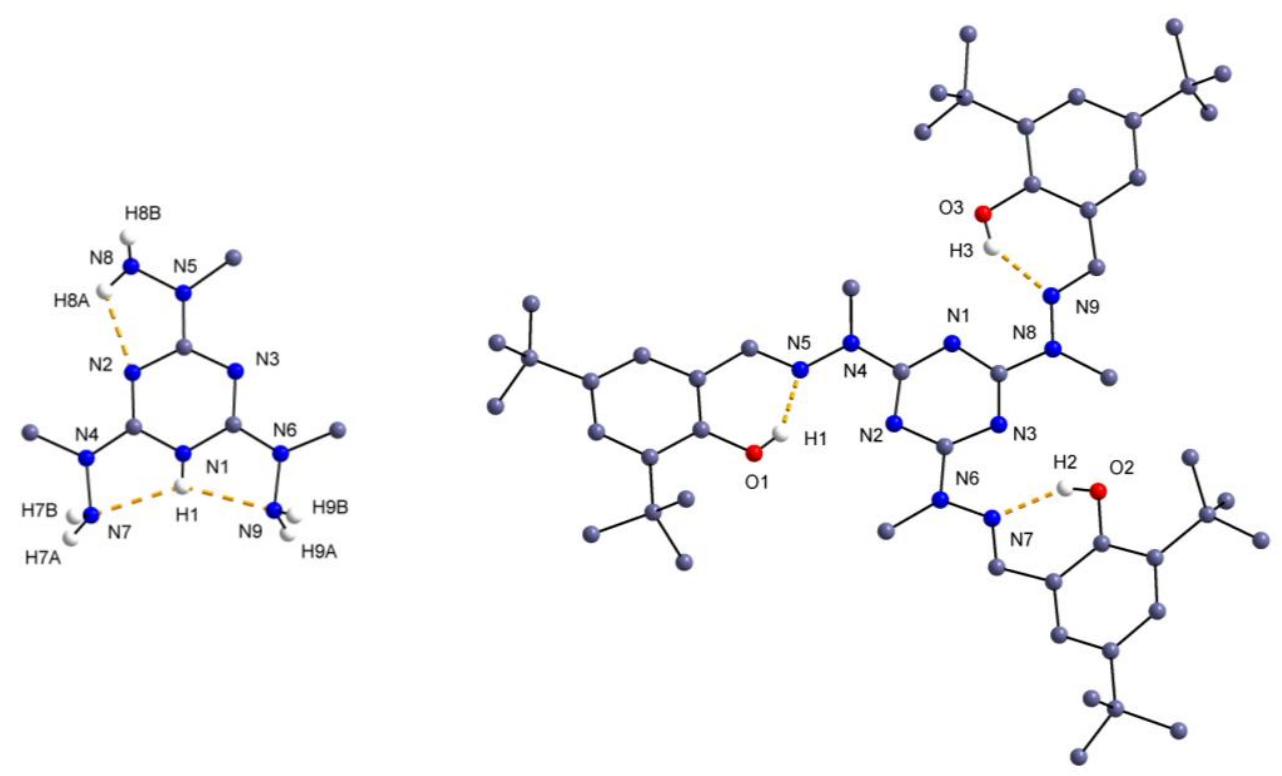

Figure 3.1. View of the molecular structures of $\mathbf{1}$ (left) and $\mathbf{H}_{3} \mathbf{L}^{\mathbf{5}}$ (right). In case of $\mathbf{1}$ counter ion is omitted for clarity, and for ligand $\mathbf{H}_{3} \mathbf{L}^{\mathbf{5}}$, most of the solvent molecules and hydrogen atoms are omitted for clarity. Selected atom distances $(\AA)$ for 1: N1-C1 1.3623(2), N1-C3 1.3675(3), N3-C3 1.3194 (2), C2-N3 1.3592(2), C2-N2 1.3580(3), C1-N2 1.3184(2). Selected atom distances $(\AA)$ for $\mathbf{H}_{3} \mathbf{L}^{\mathbf{5}}$. N1-C1 1.3424, N2-C2 1.3327, N3-C3 1.3375, N5-C5 1.2865, N9-C37 1.2737, N7-C21 1.2897, O1-H1 0.82, O2-H2 0.82, O3-H3 0.82.

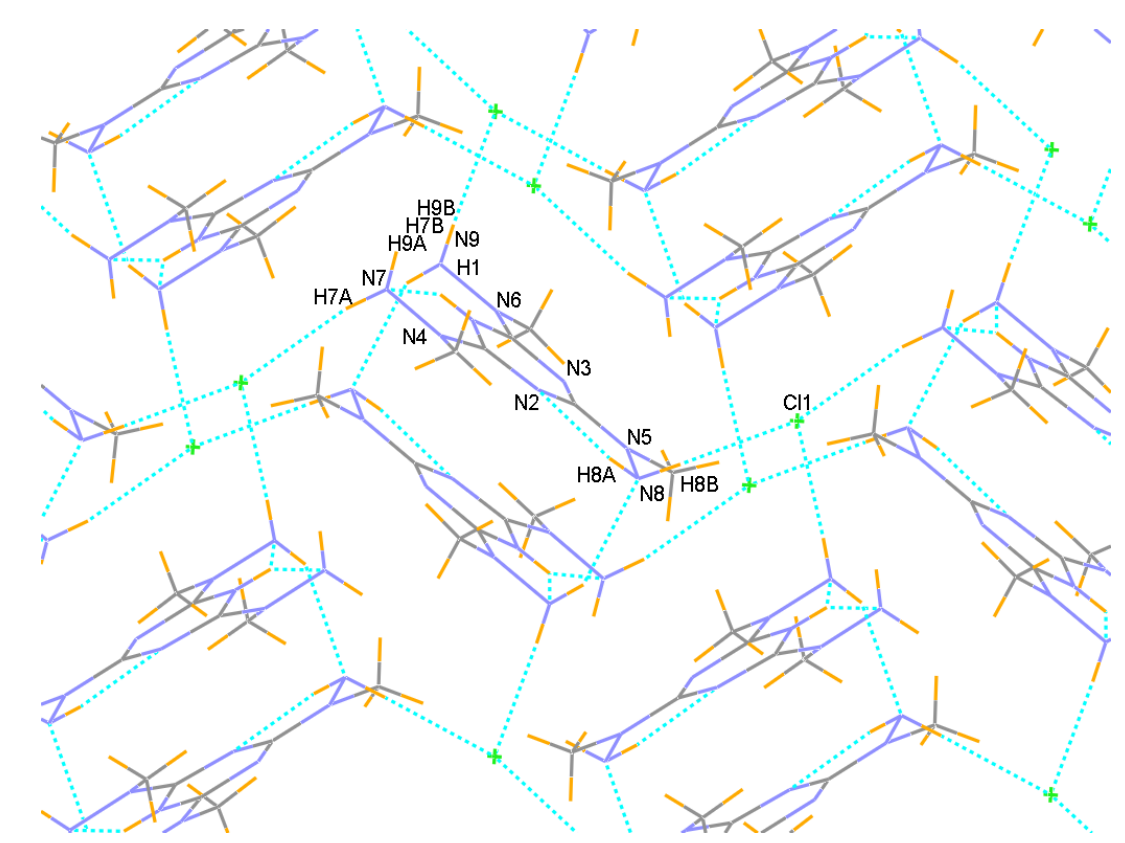

Figure 3.2. Plot of the hydrogen bonding interactions in 1. Selected intermolecular distances $(\AA)$ and angles

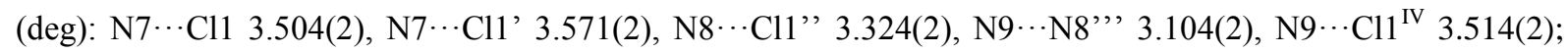

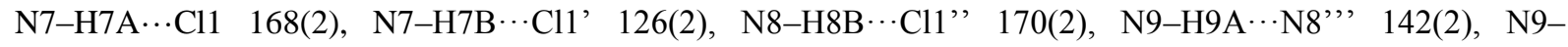
H9B $\cdots \mathrm{Cl1}^{\mathrm{IV}} 177(2)$. Symmetry transformations used to generate equivalent atoms ('): $x, 1.5-y, z-1 / 2 ;(') x, y,-$ $1+z ;\left(\right.$ ',') $1-x, 1-y, 1-z ;\left({ }^{\mathrm{IV}}\right) 1-x, y+1 / 2,1.5-z$. 


\subsection{Synthesis and Structural Characterisation of Metal Complexes}

Initially we attempted to synthesize triazine-based trimetallic complexes with ligands $\mathbf{H}_{\mathbf{3}} \mathbf{L}^{\mathbf{1}}$, and $\mathbf{H}_{3} \mathbf{L}^{2}$ although none of them yielded the desired product. A plausible reason is the hydrolysis of triazine core in presence of transition metal salts, and formation of some undesired hydrolysed product which were almost impossible to characterise by elemental analysis, mass spectrometry, or some other spectroscopic studies. Thus we avoided these ligands, and employed schiff-base type ligands $\mathbf{H}_{3} \mathbf{L}^{3}, \mathbf{H}_{6} \mathbf{L}^{4}, \mathbf{H}_{3} \mathbf{L}^{5}$ and $\mathbf{H}_{6} \mathbf{L}^{6}$ that may be useful to open new perspectives in several of these directions. Firstly, the chelate substituents attached to the 2,4,6-positions, together with the $\mathrm{N}$ atoms of the central 1,3,5-triazine core in $\mathbf{H}_{3} \mathbf{L}^{3}$ (or $\mathbf{H}_{3} \mathbf{L}^{5}$ ) establish three potentially tridentate $\{\mathrm{N}, \mathrm{N}, \mathrm{O}\}$ coordination pockets that were anticipated to enforce metal binding by all of the triazine-N; in $\mathbf{H}_{3} \mathbf{L}^{4}$ (or $\mathbf{H}_{3} \mathbf{L}^{6}$ ) the dangling $R$ $=-\mathrm{CH}_{2} \mathrm{CH}_{2} \mathrm{OH}$ groups might even provide a fourth donor site for each metal. Hence triazinebased trimetallic complexes with strong magnetic coupling and a high-spin ground state might become accessible with such ligand scaffolds. Secondly, phenol groups have been chosen as terminal donors, since the resulting metal phenolates might act as redox-active moieties, in addition to the central triazine. It should be metioned that due to solubility problem of the ligand $\mathbf{H}_{3} \mathbf{L}^{3}$, and $\mathbf{H}_{6} \mathbf{L}^{4}$, abstain those ligands in this work, and research focoused on metal complexes with ligand $\mathbf{H}_{3} \mathbf{L}^{\mathbf{5}}$, and $\mathbf{H}_{\mathbf{6}} \mathbf{L}^{\mathbf{6}}$. Decoration of the phenol groups with bulky peripheral substituents (tert-butyl) in $\mathbf{H}_{3} \mathbf{L}^{5}$ and $\mathbf{H}_{6} \mathbf{L}^{6}$ serves several purposes, namely increasing solubility, avoiding metal-bridging of the phenolate-O, and preventing unwanted radical reactivity of phenoxyl radical species. Here we attempted initial studies towards the coordination chemistry of these new ligands, which turned out to differ from our expectations and to strongly depend on the choice of the metal ion. Three unusual complexes, binuclear $\left(\mathrm{Cu}^{2+}\right)$ and tetranuclear $\left(\mathrm{Mn}^{3+}\right.$ or $\left.\mathrm{Fe}^{3+}\right)$ were obtained and thoroughly characterized, where in all cases the triazine framework had undergone partial hydrolysis to give new ligands $\mathbf{H}_{\mathbf{3}} \mathbf{L}^{\mathbf{5 a}}$ and $\mathbf{H}_{5} \mathbf{L}^{\mathbf{6 a}}$ (Scheme 3.4). 


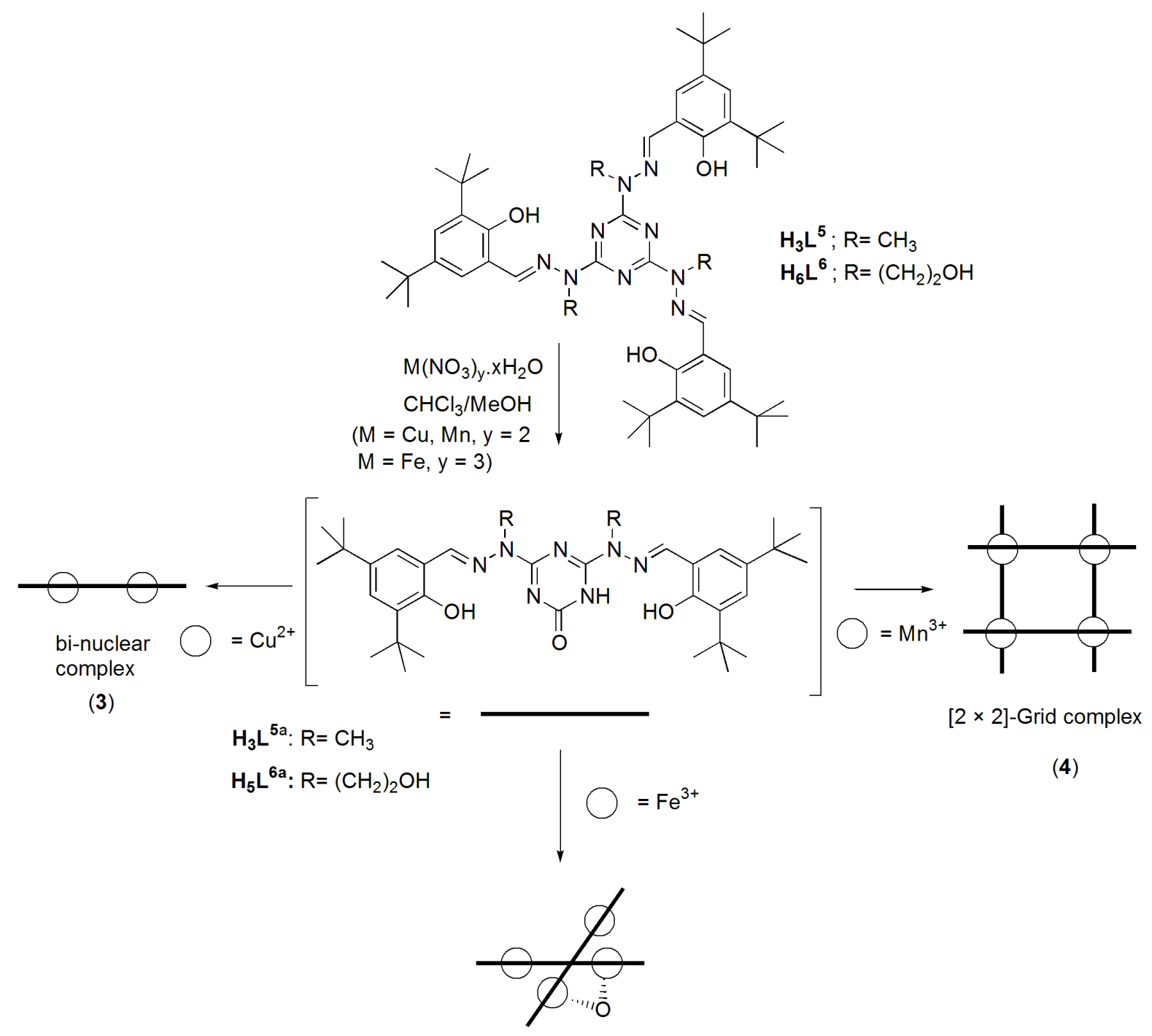

Oxo-bridged tetranuclear complex

(5)

Scheme 3.4. Self-assembly of the binuclear, and tetra nuclear complexes through an in situ ligands hydrolysis reaction.

3.3.1. Dinuclear $\mathrm{Cu}(\mathrm{II})$ complex: The reaction of $\mathbf{H}_{\mathbf{3}} \mathbf{L}^{\mathbf{5}}$ with 3 equivalents of $\mathrm{Cu}\left(\mathrm{NO}_{3}\right)_{2} \cdot 3 \mathrm{H}_{2} \mathrm{O}$ and 3 equivalents of $\mathrm{NEt}_{3}$ in a chloroform/methanol mixture (10:1) yielded a deep green solution, and removal of the solvent left a green crystalline material. Dark green crystals of complex 3 were obtained by slow evaporation of a methanol solution of the crude material, X-ray crystallographic analysis revealed the molecular structure (Figure 3.3). 


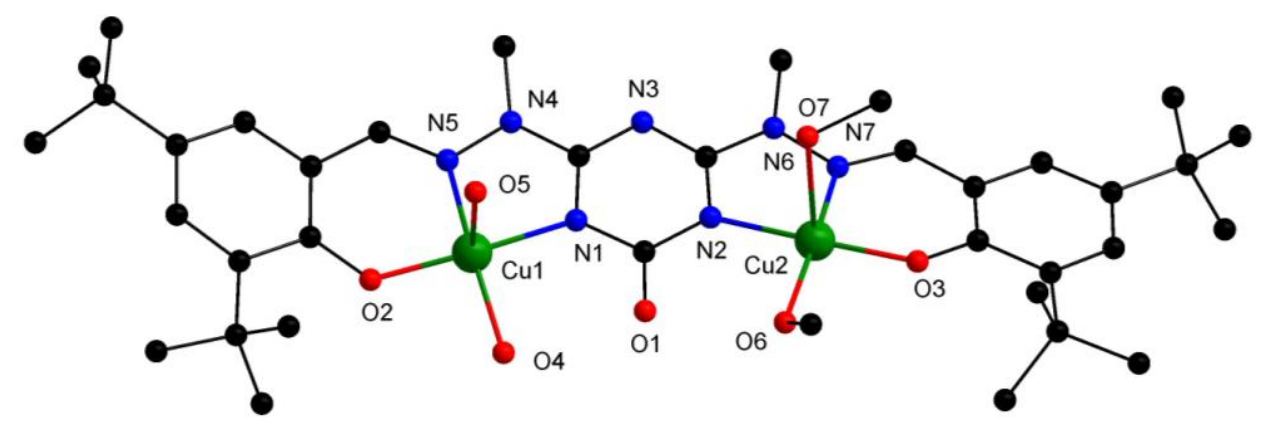

Figure 3.3. View of the molecular structure of the cation of 3. Solvent molecules, counter ions, and hydrogen atoms are omitted for clarity. Only one of the two crystallographically independent cations is shown. Selected atom distances (̊̊): Cu1-O2 1.8593(3), Cu1-N5 1.9493(4), Cu1-N1 1.9726(3), Cu1-O4 2.0022(3), Cu1-O5 2.4185(4), Cu2-O3 1.8680(3), Cu2-N7 1.9288(4), Cu2-N2 1.9674(3), Cu2-O6 1.9845(3), Cu2-O7 2.4553(3), $\mathrm{Cu} 1-\mathrm{Cu} 2$ 6.0178(6), Cu1-Cu2 6.0178(6). Selected bond angles (deg): O2-Cu1-N5 93.437(2), N5-Cu1-N1 80.813(1), N1-Cu1-O4 95.472(1), N1-Cu1-O5 94.437(2), O3-Cu2-N7 93.661(2), N7-Cu2-N2 80.945(1), N2Cu2-O6 95.307(2), N2-Cu2-O7 89.829(1).

Partial hydrolysis of $\mathbf{H}_{\mathbf{3}} \mathbf{L}^{\mathbf{5}}$ has apparently taken place, yielding the triazine-derived new ligand $\mathbf{H}_{3} \mathbf{L}^{5 \mathbf{a}}$ (Scheme 3.4). In $\mathbf{H}_{3} \mathbf{L}^{\mathbf{5 a}}$ the central triazine core is still intact, but it has lost one of its chelate arms via $\mathrm{C}-\mathrm{N}$ bond cleavage and an $\mathrm{O}$ atom is found instead. In the resulting complex 3 two copper(II) ions are hosted in the two remaining ligand compartments, ligated by the phenolato-O, the hydrazine- $\mathrm{N}$, and the adjacent triazine-N. Two independent molecules are found in the crystal lattice that differ by the solvent molecules that complete the copper(II) coordination spheres: $\left[\left(\mathrm{L}^{5 \mathrm{a}}\right) \mathrm{Cu}_{2}\left(\mathrm{H}_{2} \mathrm{O}\right)_{2}(\mathrm{MeOH})_{2}\right]^{+}$(shown in Figure 3.3). In all cases the copper(II) ions are found in square-pyramidal environment with one of the solvent ligands at rather long (Jahn-Teller) distance in apical position. The other solvent ligands are located in the basal copper(II) coordination planes and, according to the $\mathrm{O} \cdots \mathrm{O}$ distances $(2.58-2.60 \AA)$, form hydrogen bonds to the triazine-bound $\mathrm{O} 1$ atom (Chart 3.1). These hydrogen atoms, however, could not be located during refinement.

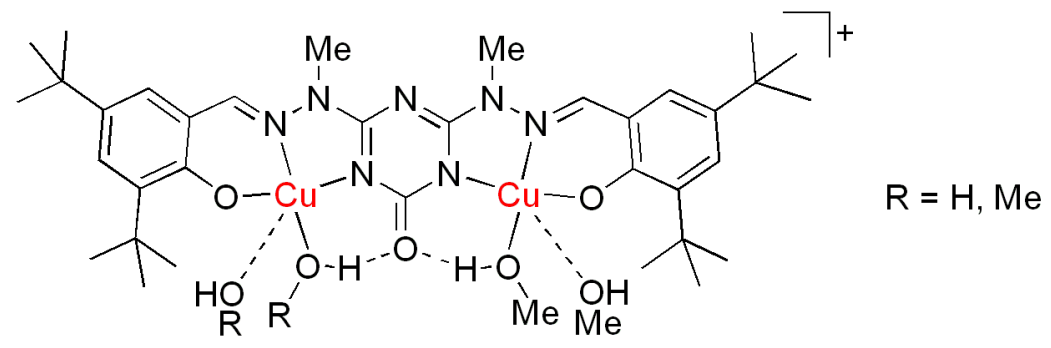

Chart 3.1. Schematic drawing of the cation of 3 . 
3.3.2. [2×2]-Grid complex of $\mathbf{M n}(\mathbf{I I I})$ : The reaction of $\mathbf{H}_{6} \mathbf{L}^{\mathbf{6}}, \mathrm{Mn}\left(\mathrm{NO}_{3}\right)_{2} \cdot \mathrm{xH}_{2} \mathrm{O}$, and $\mathrm{NEt}_{3}$ in 1:3:6 molar ratio in a chloroform/methanol mixture $(5: 2)$ under aerobic conditions afforded a red crude product, from which red crystals of the new tetranuclear complex $\left[\left(\mathrm{H}_{2} \mathrm{~L}^{6 \mathrm{a}} \mathrm{Mn}\right)_{4}\right](4)$ could be obtained by slow evaporation of an acetonitrile/methanol (2:1) solution. If the experiment is carried out with an excess of $\mathrm{Mn}\left(\mathrm{NO}_{3}\right)_{2} \cdot \mathrm{xH}_{2} \mathrm{O}$ or an excess of $\mathbf{H}_{\mathbf{6}} \mathbf{L}^{\mathbf{6}}$ under similar conditions, only a yellow amorphous products are obtained, indicating that the formation of $\mathbf{4}$ is quite sensitive to the stoichiometry of the two starting materials in this reaction. X-ray crystallography showed that compound $\mathbf{4}$ crystallizes in the monoclinic space group $C 2 / c$; the molecular structure of $\mathbf{4}$ is depicted in Figure 3.4.

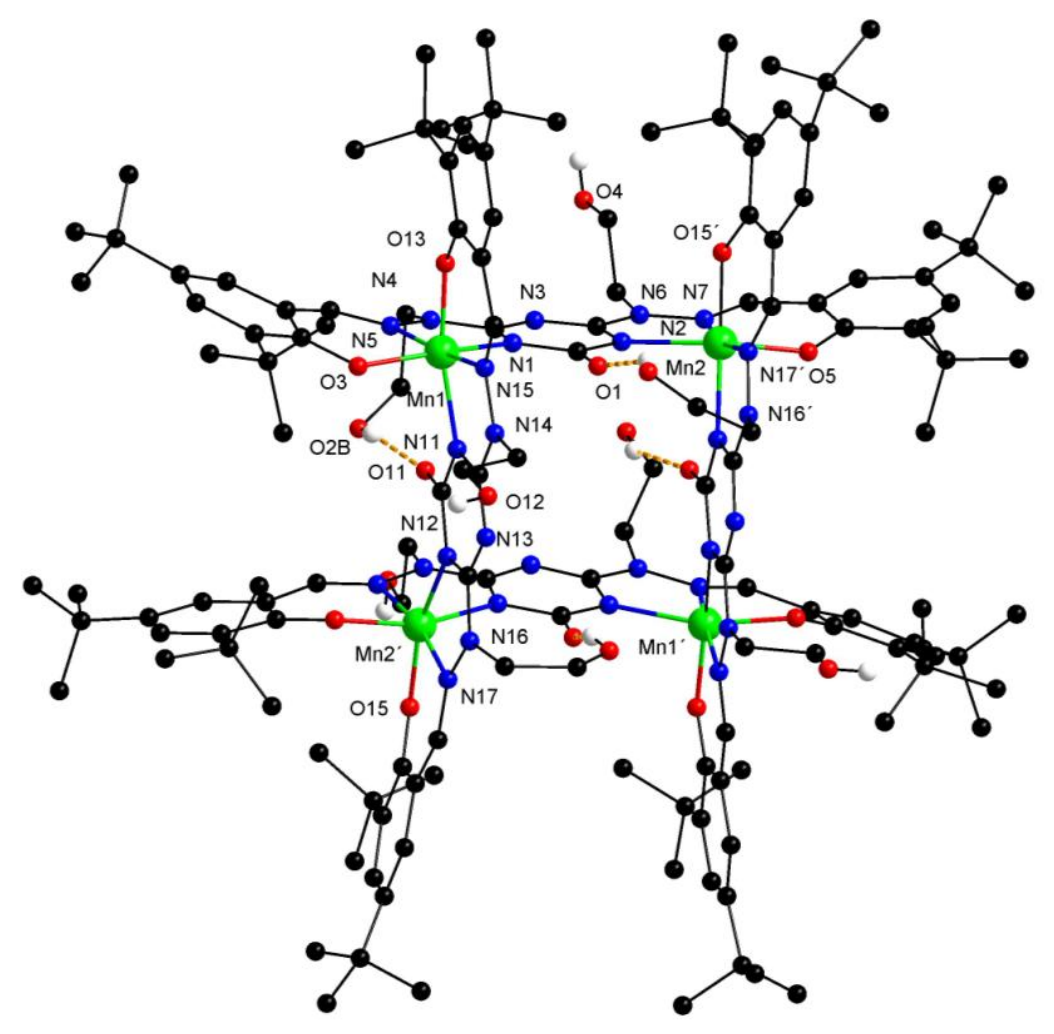

Figure 3.4. View of the molecular structure of 4. Hydrogen atoms are omitted for clarity. Symmetry transformation used to generate equivalent atoms ('): $1-\mathrm{x}, \mathrm{y}, 1.5-\mathrm{z}$.

In 4 the triazine framework of $\mathbf{H}_{\mathbf{6}} \mathbf{L}^{\mathbf{6}}$ has undergone the same hydrolytic degradation as observed above, viz. one of the chelate arms is replaced by an $\mathrm{O}$ atom. The resulting $\mathbf{H}_{5} \mathbf{L}^{\mathbf{6 a}}$ acts as a trianionic ligand where the $-\mathrm{CH}_{2} \mathrm{CH}_{2} \mathrm{OH}$ groups remain protonated; one of these $\mathrm{CH}_{2} \mathrm{CH}_{2} \mathrm{OH}$ groups of each ligand strand is involved in intramolecular $\mathrm{H}$-bonding to the triazine-bound $\mathrm{O} 1$ atom, the other $-\mathrm{CH}_{2} \mathrm{CH}_{2} \mathrm{OH}$ group is free dangling. This group was found to be disorderd about two positions. Metal ions have been oxidized to $\mathrm{Mn}^{\mathrm{III}}$, which prefers 
six-coordination. As a result complex 4 features a so-called [2×2]-grid structure composed of four ligand strands $\left[\mathbf{H}_{2} \mathbf{L}^{6 a}\right]^{3-}$ and four metal ions. While the Lehn group ${ }^{40}$ recently reported a triazine-based [2×2]-grid complex with $\mathrm{Co}^{\mathrm{II}}$ ions, $\mathbf{4}$ is the first such grid structure with bridging triazine-derived rings for $\mathrm{Mn}^{\mathrm{III}}$. Due to the bulky peripheral di-tert-butylphenolato groups the grid motif in $\mathbf{4}$ has a remarkable size of approximately $20 \times 20 \AA$.

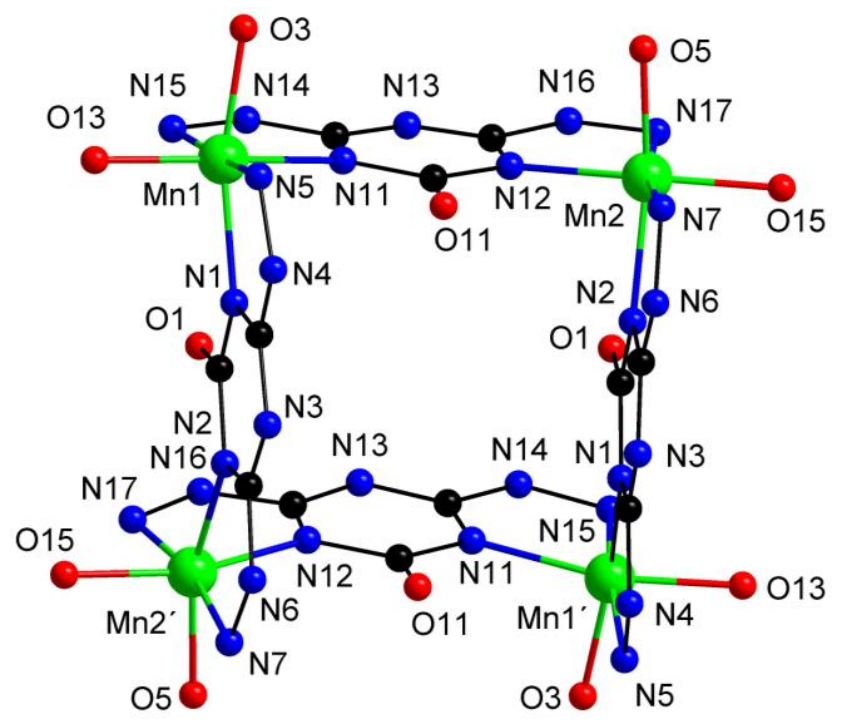

Figure 3.5. View of the central tetrametallic grid core of compound 4. Solvent molecules, counter ions, hydrogen atoms, the side arms of the aromatic ring, and N-bound hydroxyl ethyl are omitted for clarity. Selected atom distances (̊̊): Mn1-O13 1.8776(1), Mn1-O3 1.8920(1), Mn1-N11 2.0332(0), Mn1-N1 2.0472(1), Mn1-N5 2.1764(1), Mn1-N15 2.1804(1), Mn2-O15 1.8999(1), Mn2-O5 1.8822(1), Mn2-N12 2.0305(0), Mn2-N2 2.0467(1), Mn2-N7 2.1961(1), Mn1-N17 2.1726(1), Mn1-Mn2 6.0282(2), Mn1-Mn2’ 6.0762(2). Selected bond angles (deg): N1-Mn1-N5 74.991(2), N1-Mn1-O3 158.421(3), N1-Mn1-N15 110.269(2), N1-Mn1-N11 90.334(2), N1-Mn1-O13 91.700(2), N5-Mn1-O3 85.324 (2), N5-Mn1-N15 172.144, N2-Mn2-N7 75.241(2), N2Mn2-O5 158.726(3), N2-Mn2-N17’ 111.567(2), N2-Mn2-N12’ 90.374(2), N2-Mn2-O15' 91.428, N7-Mn2-O5 85.624(3), N7-Mn2-N17' 169.455. Symmetry transformation used to generate equivalent atoms ('): 1-x, y, 1. 5z.

The metal ions in $\mathbf{4}$ are located at the corners of a perfect square, and the overall grid has crystallographic $C_{2}$ symmetry (and non-crystallographic $S_{4}$ symmetry). Due to ligand constraints, and because $\mathrm{Mn}^{\mathrm{III}}$ is a strong Jahn-Teller ion, the octahedral $\left\{\mathrm{N}_{4} \mathrm{O}_{2}\right\}$ coordination environment (Figure 3.5) is quite distorted with substantial elongation along the N5-Mn1-N15 and N7-Mn2-N17 axes. Bond lengths are found in the ranges Mn- $\mathrm{N}_{\mathrm{ax}}: 2.17-2.19 \AA$; $\mathrm{Mn}-\mathrm{N}_{\mathrm{eq}}$ : $2.03-2.04 \AA$, and $\mathrm{Mn}_{-} \mathrm{O}_{\text {eq }}: 1.87-1.89 \AA$; characteristic angles are $\mathrm{N}_{\mathrm{ax}}-\mathrm{Mn}-\mathrm{N}_{\mathrm{ax}}: 170.0$ $172.1^{\circ}$ and $\mathrm{N}_{\mathrm{eq}}-\mathrm{Mn}-\mathrm{N}_{\mathrm{eq}} 88.6-96.4^{\circ}$. Interestingly, Jahn-Teller axes of all four metal ions in 4 
are roughly parallel. The distance between the triazine rings of parallel ligand strands is around $5.3 \AA$, which is considered too far for $\pi-\pi$ stacking.

3.3.3. Tetranuclear Fe(III) complex: The reaction of ligand $\mathbf{H}_{6} \mathbf{L}^{6}$, ferric nitrate and $\mathrm{NEt}_{3}$ in 1:3:6 molar ratio in methanol-chloroform mixture (2:5) afforded a black solid crude product from which block shaped black crystals of the new tetranuclear complex $\left[\mathrm{Fe}^{\mathrm{III}}{ }_{4}\left(\mu_{2^{-}}\right.\right.$ $\left.\mathrm{O})\left(\mathrm{H}_{2} \mathrm{O}\right)_{4}\left(\mathrm{H}_{2} \mathrm{~L}^{6 \mathrm{a}}\right)_{2}\left(\mathrm{~L}^{\mathrm{t}-\mathrm{Bu}}\right)_{2}\right]\left(\mathrm{NO}_{3}\right)_{2} \mathbf{5}$ was obtained by slow evaporation of an acetonitrile solution. X-ray crystallography showed that $\mathbf{5}$ crystallize in the orthorhombic space group Aba2; the molecular structure of $\mathbf{5}$ is depicted in Figure 3.6. It should be mentioned that counter anion could not be located in the refinement. IR spectroscopy, mass spectrometry, and elemental analysis suggest that two nitrate ions are the counter anionic parts of cation $\mathbf{5}$. Due to low quality of the X-ray crystallographic structure determination, metric parameters cannot be discussed in detail.

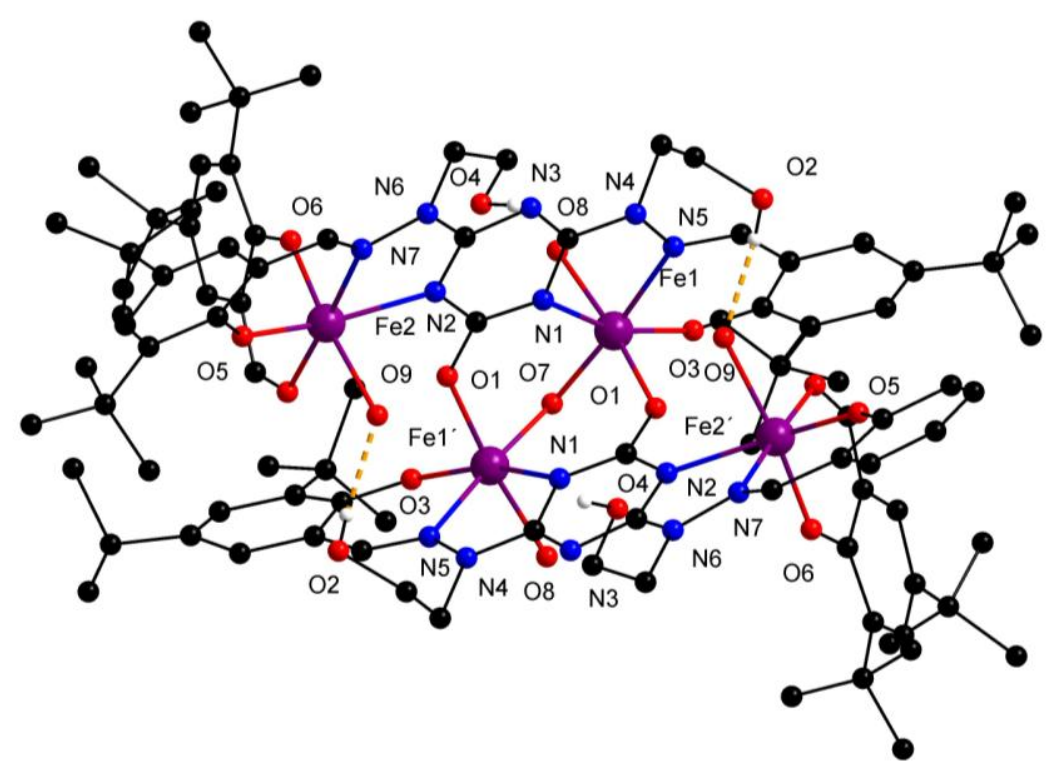

Figure 3.6. View of the molecular structure of the cation of $\mathbf{5}$. Solvent molecules, counter ions, most of the hydrogen atoms, are omitted for clarity

In $\mathbf{5}$ the triazine framework of $\mathbf{H}_{\mathbf{6}} \mathbf{L}^{\mathbf{6}}$ has undergone hydrolysis in similar manner as mentioned above, one of the chelate arms is replaced by an $\mathrm{O}$ atom and gave a new ligand $\mathbf{H}_{5} \mathbf{L}^{\mathbf{6 a}}$. In the resulting complex 5, two $\mathrm{Fe}$ (III) ions are resided in the two ligand compartments, and chelated by the phenolato-O, the hydrazine- $\mathrm{N}$, and the adjacent triazine- $\mathrm{N}$, thus formed a dinuclear complex. These two dinuclear complexes are connected by a triazine-bound $\mathrm{O}$ atom and an alkoxide $\mu_{2}-\mathrm{O}$ atom, thus giving rise to a tetranuclear cluster. Two terminal positions of the Fe atoms are capped by hydrolysed side product 3,5-di-tert-butylsalicylaldehyde $\left(\mathbf{H L}^{\mathrm{t}}{ }^{-}\right.$ 
${ }_{2}$ ). Cyrstallographically in complex 5 there are two types of Fe atoms; one side is $\mathrm{Fe}(1)$ and $\mathrm{Fe}\left(1^{\prime}\right)$ and another side is $\mathrm{Fe}(2)$ and $\mathrm{Fe}\left(2^{\prime}\right)$. Here all the Fe atoms are fashioned in hexa coordinated arrangement and exhibit a distorted octahedral geometry.

\subsection{Spectroscopy in Solution}

All new complexes were characterized by ESI mass spectrometry to clarify the stability of the complexes in solution. In order to elucidate the locous of the oxidised species of compound 3 and 4 in solution, electrochemical, spectro-electrochemical experimets, resonance Raman spectroscopy and EPR-spectroscopy were performed.

\subsubsection{Species in solution:}
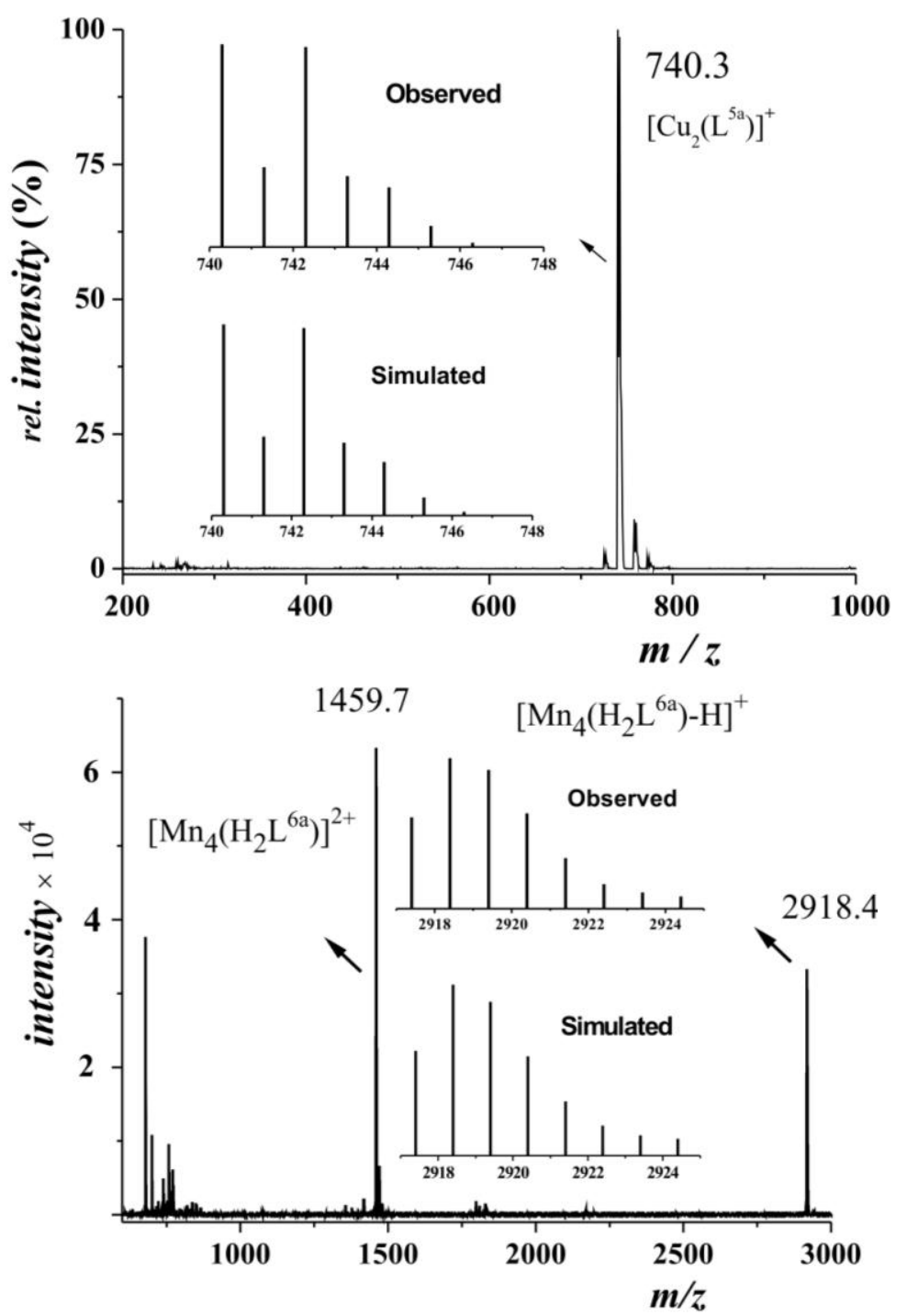

Figure 3.7. Positive ion ESI-MS spectrum of methanolic solution of 3 (top), and HRESI-MS spectrum of methanolic solution of $\mathbf{4}$ (bottom). The inset shows the observed and calculated isotopic distribution pattern for the ion $\left[\mathrm{Cu}_{2}\left(\mathrm{~L}^{5 \mathrm{a}}\right)\right]^{+}$for $\mathbf{3}$, and $\left[\mathrm{Mn}_{4}\left(\mathrm{H}_{2} \mathrm{~L}^{6 \mathrm{a}}\right)_{4}-\mathrm{H}\right]^{+}$for $\mathbf{4}$. 
Posative ion ESI-MS spectrum of methanol solution of complex $\mathbf{3}$, show molecular ion peak at $m / z, 740.3$ for $\left[\mathrm{Cu}_{2}\left(\mathrm{~L}^{5 \mathrm{a}}\right)\right]^{+}$; suggests that complex $\mathbf{3}$ is stable in solution. High-resolution (HR) ESI-MS spectrum of MeOH solution of complex 4 diplays two prominent peaks at $\mathrm{m} / \mathrm{z}$ 2918.4 and 1459.7 characteristic for singly and doubly charged ions of $\left[\mathrm{Mn}_{4}\left(\mathrm{H}_{2} \mathrm{~L}^{6 \mathrm{a}}\right)_{4}-\mathrm{H}\right]^{+}$, and $\left[\mathrm{Mn}_{4}\left(\mathrm{H}_{2} \mathrm{~L}^{6 \mathrm{a}}\right)_{4}\right]^{2+}$ implies that tetra nuclear grid-type complex stay intact in solution, and the spectrum of complex 3, and $\mathbf{4}$ are shown in Figure 3.7. Posative ion ESI-MS spectrum of acetonitrile solution of complex 5 shows three predominant peaks at $\mathrm{m} / \mathrm{z} 2115.8$, and 1027.9 characteristic for singly and doubly charged ions of $\left[\mathrm{M}-\mathrm{NO}_{3}\right]^{+}$, and $\left[\mathrm{M}-2 \mathrm{NO}_{3}+\mathrm{H}\right]^{2+}$ respectively which suggests that tetranuclear Fe(III) cluster is also intact in solution. These observations are important for analyzing the electrochemical and spectro-electrochemical experiments.

3.4.2. Electrochemistry: Cyclic voltammograms (CVs) and square-wave voltammograms (SWs) of complexes 3, and 4 have been recorded in $\mathrm{CH}_{3} \mathrm{CN}$, or THF solution respectively, containing $0.10 \mathrm{M}\left[\mathrm{N}(n-\mathrm{Bu})_{4}\right] \mathrm{PF}_{6}$ as a supporting electrolyte. Small amounts of ferrocene were added after the completion of each set of experiments as an internal standard, and all potentials are referenced to the $\mathrm{Fc}^{+} / \mathrm{Fc}$ couple and all redox potential obtained from the voltammograms are compiled in Table 3.1.

Figure 3.8 displays the CVs and SW of complex 3. On the anodic side, there is one reversible oxidation wave at $E_{1 / 2}{ }^{2}=+0.62 \mathrm{~V}$, and one irreversible oxidation wave at $E_{\mathrm{p}, \mathrm{ox}}{ }^{3}=+1.19 \mathrm{~V}$. Closer investigation of the first reversible oxidation revealed a broadening of the wave and a small inflection on both the rising part of the forward and the reverse waves. Likewise, this wave appeared to be rather broad when compared to the internal ferrocene standard in both, cyclic and square wave voltammetry. Successful simulations of square wave voltammograms required two closely spaced individual peaks at +0.587 and $0.649 \mathrm{~V}\left(\Delta E_{1 / 2}=62 \mathrm{mV}\right)$. Simulation of the experimental $\mathrm{CVs}^{41}$ likewise showed that the inflection of the rising portions of each peak can only be accommodated by two individual, closely spaced oneelectron processes. Figure 3.8d documents the agreement between the experimental and the simulated CVs. The obtained half-wave potentials of +0.583 and $+0.637 \mathrm{~V}$ are in satisfactory agreement with those obtained from Square wave voltammetry and result in essentially the same $\Delta E_{1 / 2}$ value $(54 \mathrm{mV})$. Complex 3 also displays a reduction. At low sweep rates the reduced species adsorbs to the electrode surface as is evident from the sharp peak superimposed on the reduction wave and the associated cathodic stripping peak. This renders 
the reduction wave rather ill-defined with a peak at approximately $-0.92 \mathrm{~V}$. At sweep rates of $1 \mathrm{~V} / \mathrm{s}$ or higher the reduction wave constitutes a quasi-reversible couple at $E_{1 / 2}=-0.84 \mathrm{~V}$. Its peak current is about $40 \%$ of that of the first reversible oxidation which points to a oneelectron process. The substantially larger peak-to-peak separation of $335 \mathrm{mV}$ at $v=2.0 \mathrm{~V} / \mathrm{s}$ compared to $246 \mathrm{mV}$ for the oxidation signals sluggish electron transfer kinetics because of either intrinsically slow structural reorganisation, partial electrode fouling or both.

a

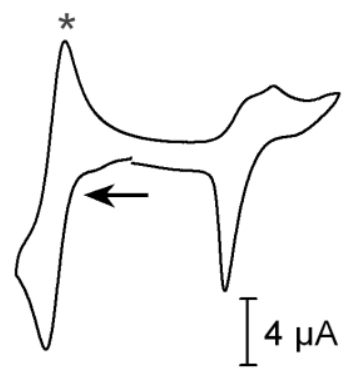

b
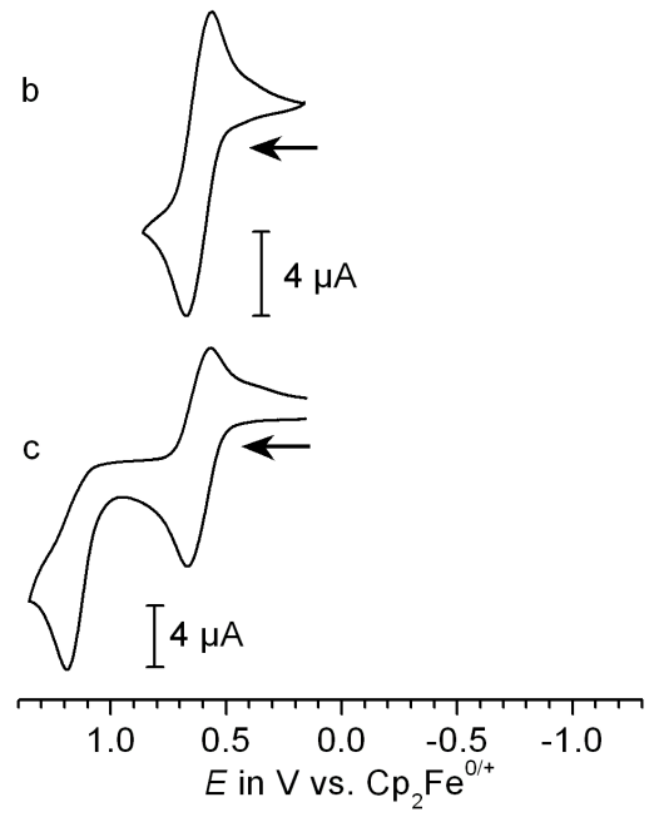

d

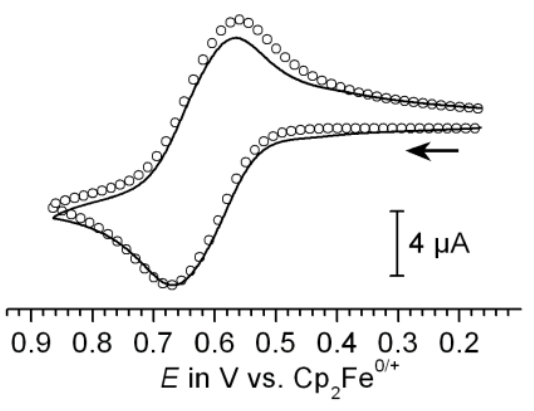

e

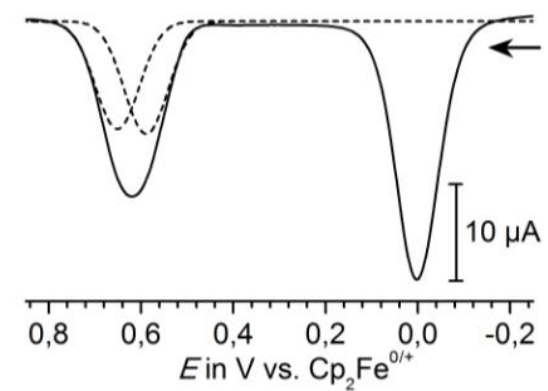

Figure 3.8. Cyclic voltammograms (in left side) of complex $\mathbf{3}$ in acetonitrile $\left.(0.1 \mathrm{M})\left[(n-\mathrm{Bu})_{4} \mathrm{~N}\right] \mathrm{PF}_{6}\right)$ at $20^{\circ} \mathrm{C}$ recorded at a glassy carbon working electrode versus a $\mathrm{Ag} / \mathrm{AgNO}_{3}$ reference electrode. (a) Reduction (*marks the internal Fc-standard), (b) $1^{\text {st }}$ oxidation, (c) $1^{\text {st }}$ and $2^{\text {nd }}$ Oxidation at $\mathrm{v}=100 \mathrm{mV} / \mathrm{s}$, and (d) comparison of reversible oxidation wave of complex 3 with experimental vs. simulated $v=100 \mathrm{mV} / \mathrm{s}$, circle and bold line indicates simulated and experimental respectively (in right side), in addition (e) square-wave voltammograms (bottom, right) of reversible oxidation wave of compound $\mathbf{3}$. 
Table 3.1. Electrochemical data of compound 3 and 4.

\begin{tabular}{|l|l|l|l|l|}
\hline & \multicolumn{3}{|c|}{ CV data $^{[\mathrm{a}]}$} & Square wave voltammetry $^{[\mathrm{a}]}$ \\
\hline Compound & $E_{1 / 2}{ }^{\mathrm{red}}[\mathrm{V}]$ & $E_{1 / 2}{ }^{\mathrm{ox}}[\mathrm{V}]^{[\mathrm{c}]}$ & $E^{3}[\mathrm{~V}]^{[\mathrm{b}]}$ & $E_{1 / 2}{ }^{2}[\mathrm{~V}]^{[\mathrm{c}]}$ \\
\hline $\mathbf{3}$ & -0.84 & $0.583,{ }^{[\mathrm{c}]} 0.637^{[\mathrm{c}]}$ & 1.19 & $0.587,0.649$ \\
\hline $\mathbf{4}$ & $-1.24^{[\mathrm{b}]}$ & $0.100,0.187$, & 0.78 & $0.092,0.180,0.287,0.365$ \\
& & $0.292,0.366$ & & \\
\hline
\end{tabular}

[a] Potentials are given relative to the $\mathrm{Fc} / \mathrm{Fc}^{+}$reference. Compound 3: $\mathrm{CH}_{3} \mathrm{CN}\left(0.1 \mathrm{M}\left[(n-\mathrm{Bu})_{4} \mathrm{~N}\right]\left(\mathrm{PF}_{6}\right)\right.$; compound 4: THF $\left(0.1 \mathrm{M}\left[(n-\mathrm{Bu})_{4} \mathrm{~N}\right]\left(\mathrm{PF}_{6}\right)\right)$ at $293 \mathrm{~K}$. [b] Peak potential for an irreversible redox process. [c] Data obtained from digital simulation of experimental CVs or from deconvolution of the experimental SWVs, respectively.

The CVs of complex 4 at scan rates $100 \mathrm{mV} / \mathrm{s}$ is depicted in Figure 3.9. Complex 4 exhibits one irreversible reduction at $E_{\mathrm{p} \text {,red }}{ }^{1}=-1.24 \mathrm{~V}$ as well as apparently two reversible oxidation waves at ca. +0.16 and $+0.33 \mathrm{~V}$.

(a)

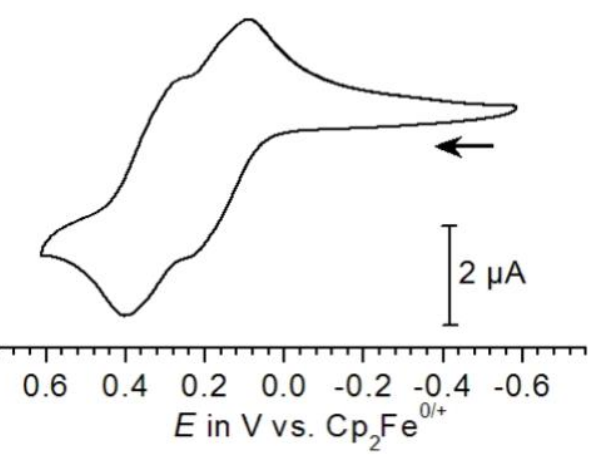

(c)

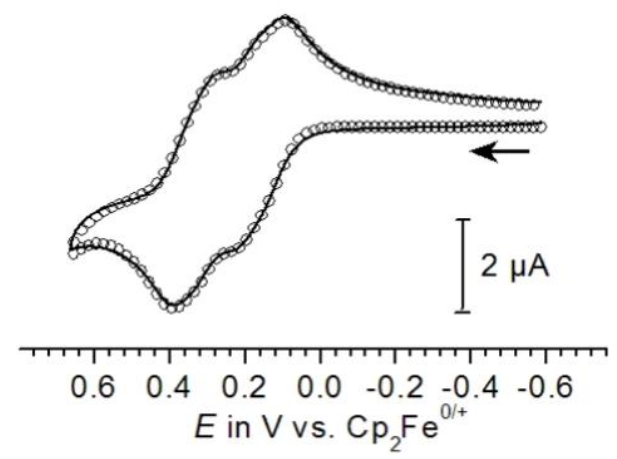

(b)
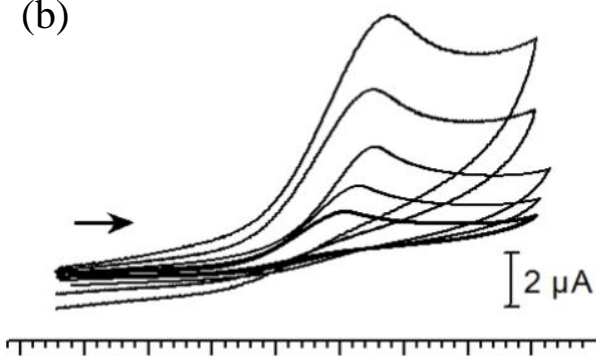

$-0.2-0.4-0.6-0.8-1.0-1.2-1.4-1.6-1.8$

$E$ in $V$ vs. $\mathrm{Cp}_{2} \mathrm{Fe}^{0 /+}$

(d)

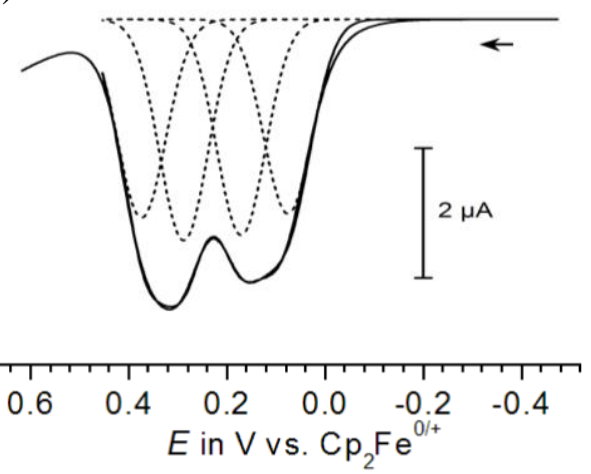

Figure 3.9. Cyclic voltammograms of complex 4 in $\operatorname{THF}(0.1 \mathrm{M})\left[(n-\mathrm{Bu})_{4} \mathrm{~N}\right]\left(\mathrm{PF}_{6}\right)$ at $20^{\circ} \mathrm{C}$ recorded with scan rate at $v=100 \mathrm{mV} / \mathrm{s}$ (a and c) or at sweep rates of 50,100, 200, 500 and $1000 \mathrm{mV} / \mathrm{s}$ (b). (c) and (d): Comparison of the experimental and simulated voltammograms of complex $\mathbf{4}$ obtained with the data in Table 2. Experimental are given as solid lines and simulated curves as open circles (c) or as broken lines (d). 
Square wave voltammetry, however, shows that the anodic behavior of $\mathbf{4}$ is even more complex. In fact, the experimental peak shape is only reproduced by envoking a total of four consecutive one-electron processes. Two data sets for the individual peak/half-wave potentials were obtained from deconvolution of the experimental SWVs and from digital simulation of the CVs are shown in Figure 3.9c and 3.9d. Individual data sets differ by no more than $8 \mathrm{mV}$ and agree with the presence of two pairs of two closely spaced $\left(\Delta E_{1 / 2}=74-88 \mathrm{mV}\right)$ oneelectron waves with some larger spacing of 105-107 $\mathrm{mV}$ between the second and third redox processes. The total four-electron nature of the composite anodic waves was further corroborated by a combination of linear sweep voltammetry and chronocoulometry according to the method of Baranski et al. ${ }^{42}$

The two or four oxidations of complexes $\mathbf{3}$ and $\mathbf{4}$ could, in principle, originate from either metal-based ( $\mathrm{n} \mathrm{M}^{\mathrm{n}+} / \mathrm{M}^{(\mathrm{n}+1)+}$ ) or ligand-based ( $\mathrm{n}$ times phenolate/phenoxy radical) couples as is sketched in Schemes 3.5 and 3.6 respectively. In order to resolve this ambiguity and to identify the oxidation sites in the two complexes, UV/Vis/NIR spectroelectrochemical measurements were conducted.

$$
\begin{array}{ll}
\text { (a) }\left[\mathrm{Cu}_{2}{ }_{2}\left(\mathrm{~L}^{5 \mathrm{a}}\right)\right]^{+} & \stackrel{-2 \mathrm{e}^{-}}{\rightleftharpoons}\left[\mathrm{Cu}_{2}{ }_{2}\left(\mathrm{~L}^{5 \mathrm{a}}\right)\right]^{3+} \\
\text { (b) }\left[\mathrm{Cu}_{2}{ }_{2}\left(\mathrm{~L}^{5 \mathrm{a}}\right)\right]^{+} & \stackrel{-2 \mathrm{e}^{-}}{\rightleftharpoons}\left[\mathrm{Cu}_{2}{ }_{2}\left(\mathrm{~L}^{5 \mathrm{a}} \bullet \bullet\right)\right]^{3+}
\end{array}
$$

Scheme 3.5. Proposed redox properties scheme of compound 3; (a) metal based two electrons oxidation, and (b) ligand based two electrons oxidation.

$$
\begin{aligned}
& \text { (a) }\left[\mathrm{Mn}_{4}{ }_{4}^{\mathrm{III}}\left(\mathrm{H}_{2} \mathrm{~L}^{6 \mathrm{a}}\right)\right] \stackrel{-2 \mathrm{e}^{-}}{\rightleftharpoons}\left[\mathrm{Mn}_{2}^{\mathrm{III}}{ }_{2} \mathrm{Mn}^{\mathrm{IV}}{ }_{2}\left(\mathrm{H}_{2} \mathrm{~L}^{6 \mathrm{a}}\right)\right]^{2+} \stackrel{-2 \mathrm{e}^{-}}{\rightleftharpoons}\left[\mathrm{Mn}^{\mathrm{IV}}{ }_{4}\left(\mathrm{H}_{2} \mathrm{~L}^{6 \mathrm{a}}\right)\right]^{4+} \\
& +2 \mathrm{e}^{-}+2 \mathrm{e}^{-} \\
& \text {(b) }\left[\mathrm{Mn}^{\mathrm{III}}{ }_{4}\left(\mathrm{H}_{2} \mathrm{~L}^{6 \mathrm{a}}\right)\right] \underset{+2 \mathrm{e}^{-}}{\rightleftharpoons}\left[\mathrm{Mn}^{\mathrm{III}}{ }_{4}\left(\mathrm{H}_{2} \mathrm{~L}^{6 \mathrm{a}} \bullet \bullet\right)\right]^{2+} \underset{+2 \mathrm{e}^{-}}{\rightleftharpoons}\left[\mathrm{Mn}^{\mathrm{III}}{ }_{4}^{-}\left(\mathrm{H}_{2} \mathrm{~L}^{6 \mathrm{a}} \bullet \bullet \bullet \bullet\right)\right]^{4+}
\end{aligned}
$$

Scheme 3.6. Proposed redox properties scheme of compound 4; (a) metal based four electrons oxidation, and (b) ligand based four electrons oxidation. 
3.4.3. Spectroelectrochemistry: UV-vis spectra were recorded in situ during electrolysis. This allowed for the progress of the oxidation to be followed and the electronic spectra of the oxidized complexes to be measured.

Experiments on 3 were performed in $\mathrm{MeCN}$ and in $\mathrm{MeNO}_{2}$. The experiments were complicated by the rather low solubility of $\mathbf{3}$ and by slow decomposition of the oxidized forms. Neutral 3 shows three absorption bands in the range between 200 and $1200 \mathrm{~nm}$. The high energy bands at 324 and $424 \mathrm{~nm}$ originate from $\pi-\pi^{*}$ transition of the coordinated phenolic chromophores, while the low energy band at $1126 \mathrm{~nm}$ is the $\mathrm{d}-\mathrm{d}$ transition of the $\mathrm{d}^{9}$ $\mathrm{Cu}$ (II) ions. UV-vis spectra recorded during the electrolysis are depicted in Figure 3.10. Stepwise oxidation is accompanied by a blue shift of the prominent UV band from 324 to 305 $\mathrm{nm}$, an intensity decrease of the asymmetric band at $424 \mathrm{~nm}$ and the growth of a new weaker band at $510 \mathrm{~nm}$. Of particular note is the appearance of a broad feature near $1070 \mathrm{~nm}$. Closer inspection shows that this band is composed out of two underlying sub-bands. It is quite revealing and consistent with the presence of two individual, closely spaced one-electron processes that the relative areas of these sub-bands change during stepwise conversion to the fully oxidized dication. Thus, at early stages the lower energy component at $1140 \mathrm{~nm}$ dominates while the higher energy feature appears at ca. $920 \mathrm{~nm}$. In the final state the higher energy component dominates and has shifted to ca. $980 \mathrm{~nm}$ while the low energy component maintains its position. Due to the proximity of the two underlying oxidation waves partially oxidized samples necessarily contain a mixture of the reduced $\mathbf{3}$, monooxidized $\mathbf{3}^{+}$and dioxidized $\mathbf{3}^{2+}$ forms. The changing appearance of the low-energy band with progressive oxidation can thus be explained by slight differences in the intrinsic absorption profiles of monooxidised dication and the dioxidized trication in that spectral region. We can, however, not exclude that substitution of the aquo and $\mathrm{MeOH}$ ligands by acetonitrile contribute to the changing absorption profile in that spectral region. Indeed, back reduction resulted in the overall recovery of the spectrum of $\mathbf{3}$ but with a slight shift of the visible band from $424 \mathrm{~nm}$ to $418 \mathrm{~nm}$. Results in $\mathrm{CH}_{3} \mathrm{NO}_{2} /\left[n-\mathrm{Bu}_{4} \mathrm{~N}\right]\left[\mathrm{PF}_{6}\right]$ are grossly similar to those in $\mathrm{CH}_{3} \mathrm{CN}$. They show a more uniform growth and decrease of the composite low-energy band but a split higher energy band with peaks at 425 and $407 \mathrm{~nm}$ after a full oxidation-reduction cycle instead of just the $425 \mathrm{~nm}$ one of pristine $\mathbf{3}$, which may again point to ligand substitution processes. The same spectral features were observed during chemical oxidation upon addition of 2 equivalents of ceric ammonium nitrate to the $\mathrm{CH}_{3} \mathrm{CN}$ solution of compound 3 at $-78^{\circ} \mathrm{C}$. The oxidised species proved to be rather unstable and decayed within 10 minutes even at $-78^{\circ} \mathrm{C}$. Pertinent to the question about the identitiy of the redox sites involved in the anodic processes 
of $\mathbf{3}$ is the $510 \mathrm{~nm}$ band of oxidized $\mathbf{3}^{\mathrm{nt}}$. This feature is reminiscent of the phenolate-to-copper (III) charge transfer (CT) transition, ${ }^{36 a-c}$ which argues for metal centred oxidation processes.
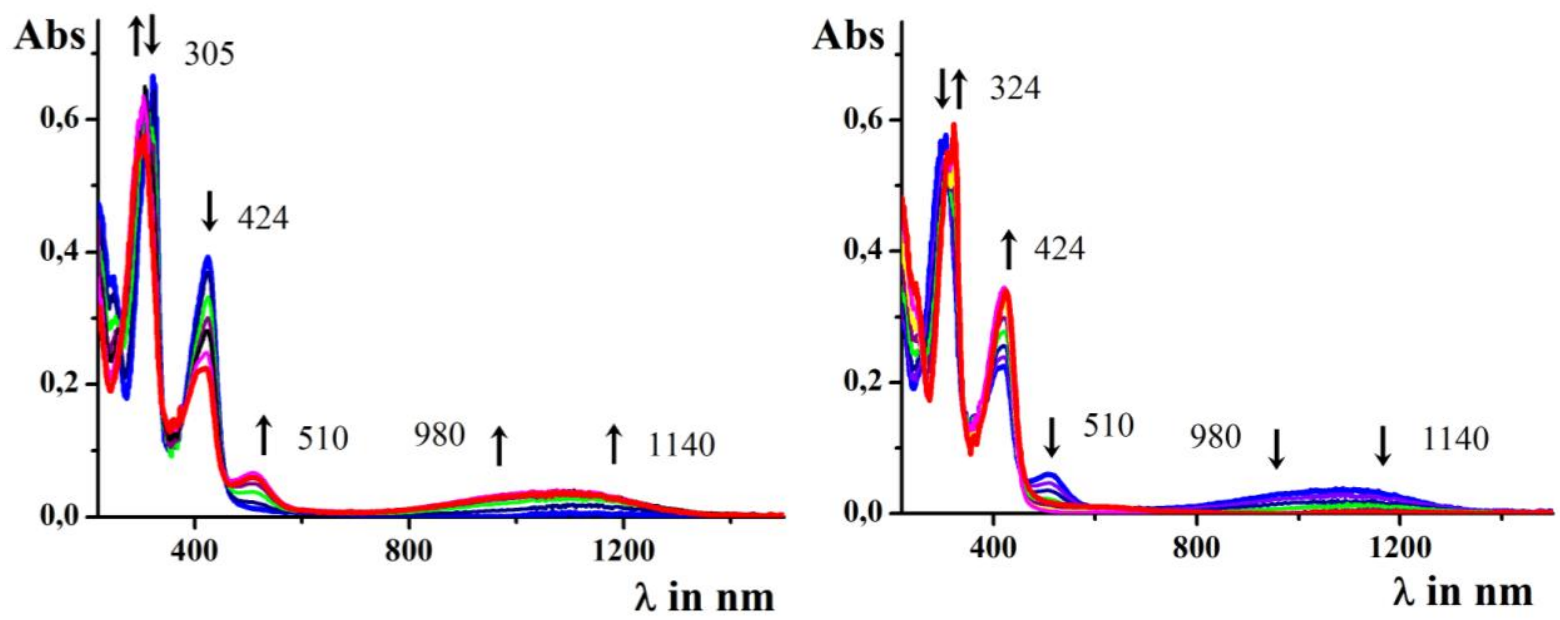

Figure 3.10. Spectroscopic changes upon stepwise oxidation $\left(\mathrm{CH}_{3} \mathrm{CN} / \mathrm{NBu}_{4} \mathrm{PF}_{6}\right)$ of $\mathbf{3}$ to $\mathbf{3}^{3+}$ (left) and upon stepwise reduction of $\mathbf{3}^{3+}$ (right). The blue and red bold line denotes spectrum of the starting complex, and oxidised complex respectively.

Table 3.2. Electronic Properties of Compounds 3 and 4.

\begin{tabular}{|l|l|}
\hline compound & $\lambda_{\max }[\mathrm{nm}]\left(\varepsilon\left[10^{4} \mathrm{M}^{-1} \mathrm{~cm}^{-1}\right]\right)^{[\mathrm{a}]}$ \\
\hline $\mathbf{3}$ & $324(0.4621), 424(0.2754), 1126(0.0069)$ \\
\hline$[3]^{2+}$ & $305(0.4043), 424(0.224), 510(0.1549), 980(0.0234), 1140(0.0251)$ \\
\hline $\mathbf{4}$ & $\begin{array}{l}259(0.3863), 318(0.3594), 367 \mathrm{sh}(0.1763), 400 \mathrm{sh}(0.1507), 458 \mathrm{sh}(0.0763) \\
740(0.00568)\end{array}$ \\
\hline$[4]^{4+}$ & $\begin{array}{l}259(0.2966), 325(0.4870), 377 \mathrm{sh}(0.1999), 434 \mathrm{sh}(0.0800), 473 \mathrm{sh}(0.0608), \\
560(0.0988), 670(0.040), 846(0.029), 1094(0.0180)\end{array}$ \\
\hline
\end{tabular}

[a] UV/Vis spectra recorded in $\mathrm{CH}_{3} \mathrm{CN}-1,2 / \mathrm{NBu}_{4} \mathrm{PF}_{6}$, and THF-1,2/NBu $\mathrm{PF}_{6}(0.1 \mathrm{M})$ at $298 \mathrm{~K}$.

UV/Vis/NIR spectroelectrochemical measurements of complex 4 were recorded in THF/0.2 $\mathrm{M}\left[n-\mathrm{Bu}_{4} \mathrm{~N}\right]\left[\mathrm{PF}_{6}\right]$ (Figure 3.11). Bands at 259, 318, 400 and $458 \mathrm{~nm}$ originate from $\pi-\pi^{*}$ transitions of the phenolic chromophores, while weak low energy bands at 740 and $1360 \mathrm{~nm}$ are assigned as $\mathrm{d}-\mathrm{d}$ transitions of the $\mathrm{d}^{4} \mathrm{Mn}^{3+}$ ions. Slow oxidation did not allow for a resolution of the close-lying individual oxidations. This is because potential differences are too small and none of the intermediate redox states between $\mathbf{4}$ and $\mathbf{4}^{4+}$ has an own characteristic absorption. Thus, only the gross changes from 4 to $4^{4+}$ can be discussed. 


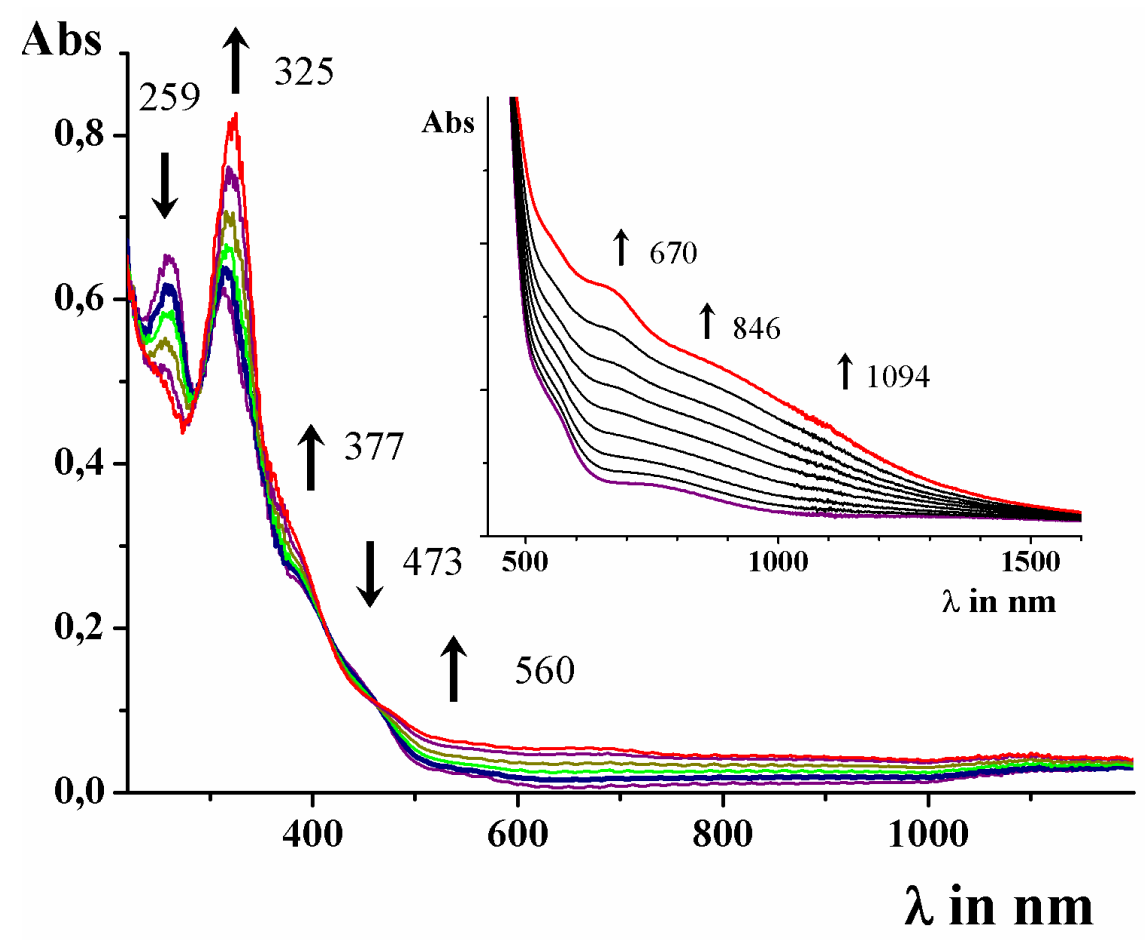

Figure 3.11. Spectroscopic changes upon stepwise oxidation (THF/NBu $\left.4 \mathrm{PF}_{6}\right)$ of complex 4 to $\mathbf{4}^{4+}$. The inset shows the spectrum of low energy part of the spectrum. The blue and red line denotes spectrum of the starting complex, and oxidised complex respectively.

Upon increase of the voltage, broad unstructured absorptions appear in the 500 to $1000 \mathrm{~nm}$ range. Individual peak positions can be extracted by spectrum deconvolution but must be regarded with all necessary caution inherent to such procedures. Deconvoluted peaks at 846, 670 and $560 \mathrm{~nm}$ are closely similar to those for $\mathrm{Mn}^{\mathrm{IV}}$-phenolate from $\mathrm{Mn}$ (salen), ${ }^{43}$ thus oxidised species of the compound $\mathbf{4}$ can be assigned as $\mathrm{Mn}^{\mathrm{IV}}$ species.

3.4.4. EPR spectroscopy: The $X$-band EPR spectrum of compound 3 in $\mathrm{CH}_{3} \mathrm{CH}_{2} \mathrm{CN}$ have been recorded at $145 \mathrm{~K}$. It display a signal at $g=2.07$ which is characteristic for typical $\mathrm{Cu}^{\mathrm{II}}$ complex $^{36}$ (Figure 3.12), and the EPR spectrum of compound [3] ${ }^{2+}$ in $\mathrm{CH}_{3} \mathrm{CH}_{2} \mathrm{CN}$, generated chemically by 2 equivalent ceric ammonium nitrate, and have been recorded at $145 \mathrm{~K}$. The spectrum is showed in Figure 3.12. Oxidation of 3 is found an EPR silent species ${ }^{36 a}(<10 \%$ of their original intensity at $145 \mathrm{~K} ; g=2.08$ ), that can be ratinalised by either phenoxyl radicals which is antiferromagnetically coupled to $\mathrm{Cu}^{\mathrm{II}}$ ion $(S=1 / 2)$ individually, or high valent low spin $\mathrm{Cu}^{\mathrm{III}}$ species. Thus further spectroscopic analysis (say, resonance Raman spectroscopy, X-ray absorption spectroscopy) will be needed to clarify the electronic properties of the oxidised species of compound $\mathbf{3}$. 


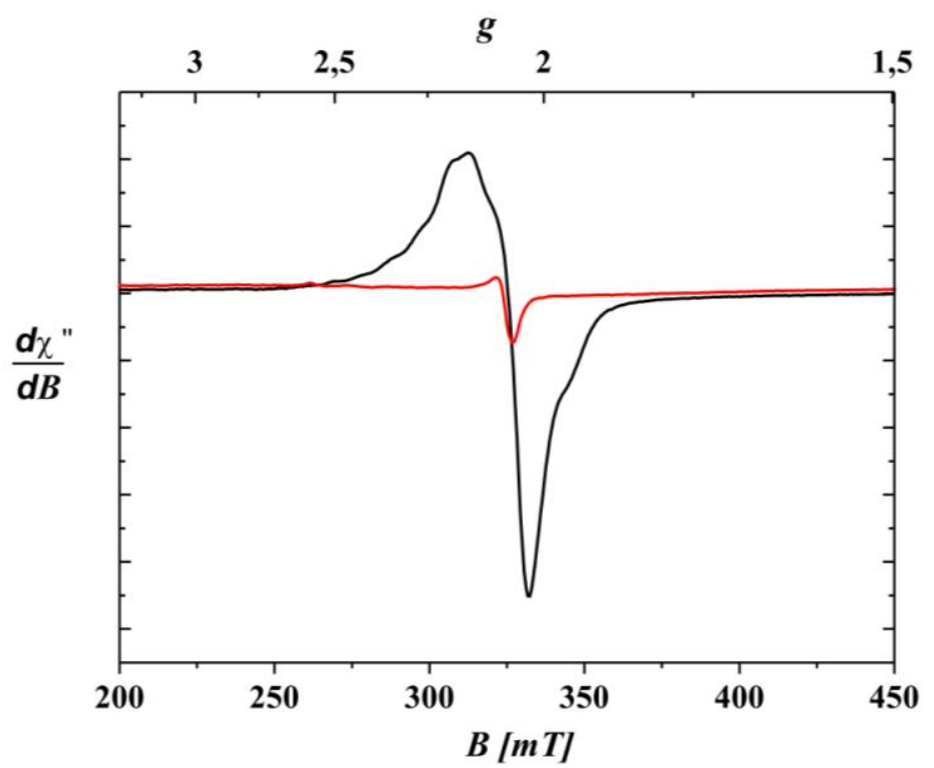

Figure 3.12. X-band EPR sectrum of 3 (in black line), and $[3]^{2+}$ (red line) in $\mathrm{CH}_{3} \mathrm{CH}_{2} \mathrm{CN}(1 \mathrm{mM})$ at $145 \mathrm{~K}$.

The X-band EPR spectrum of compound $\mathbf{4}$ in THF at low temperature has been recorded, but no significant $\mathrm{Mn}^{\mathrm{III}}$ signal was observed. Furthermore we attempted to measure the chemically oxidised species of 4 by $\mathrm{AgX}\left(\mathrm{X}=\mathrm{SbF}_{6}, \mathrm{BF}_{4}\right.$, OTf) in THF (or in $\mathrm{CH}_{2} \mathrm{Cl}_{2} / \mathrm{OEt}_{2}$ mixture), the spectrum showed typical reduced species of $\mathrm{Mn}^{\mathrm{II}}$ through partial decomposition.

3.4.5. Resonance Raman (rR) Spectroscopy: The resonance Raman spectroscopy (rR) is particularly useful for gaining insight into the structural and electronic properties of both metal-phenoxyl radical as well as metal-phenolate species, and moreover, to distinguish between them. ${ }^{44}$ Laser excitation into the $\pi-\pi^{*} \mathrm{UV} / \mathrm{vis}$ band $\left(\lambda_{\max } \sim 400 \mathrm{~nm}\right)$ of mono- and bis(phenoxyl radical) copper(II) complexes - prepared by chemical or electrochemical oxidation of those copper-phenolate compounds - resulted in resonance Raman spectra very similar to those of free phenoxyl radicals, ${ }^{36}$ with two dominant features at $\sim 1500 \mathrm{~cm}^{-1}\left(v_{7 \mathrm{a}}\right.$, predominantly C-O stretching) and $\sim 1600 \mathrm{~cm}^{-1}$ ( $v_{8 \mathrm{a}}, \mathrm{C}_{\text {ortho }}-\mathrm{C}_{\text {meta }}$ ring stretching). The $v_{7 \mathrm{a}}$ band in these complexes occurs at $\sim 250 \mathrm{~cm}^{-1}$ higher energy than in the metal-phenolate precursors, indicative of increased $\mathrm{C}-\mathrm{O}$ double bond character in the coordinated phenoxyl radicals. The Raman spectra of the copper-phenolate complexes generally show a set of multiple features between 1100 and $1600 \mathrm{~cm}^{-1}$.

The rR spectrum of oxidised 3 in $500-1800 \mathrm{~cm}^{-1}$ range obtained in $\mathrm{CH}_{3} \mathrm{CN}$ at $-40^{\circ} \mathrm{C}$ exhibited new bands at 726, 1048, 1182, 1280, 1570, and $1646 \mathrm{~cm}^{-1}$ (Figure 3.13), which corresponds to phenolate-to- $\mathrm{Cu}(\mathrm{III})$ transition. ${ }^{36 \mathrm{e}}$ The energy of 1048 , and $1182 \mathrm{~cm}^{-1}$ are assigned as in- plane aromatic modes, and the energy of 1280 and $1570 \mathrm{~cm}^{-1}$ are assigned as $\mathrm{C}-\mathrm{O}$ stretching and 
$\mathrm{C}_{\text {ortho }}-\mathrm{C}_{\text {meta }}$ ring stretching vibrations respectively. Thus from the $\mathrm{rR}$ spectroscopy it is evident that oxidised $\mathbf{3}$ is bis-copper (III) species and not bis(phenoxyl radical)copper(II).

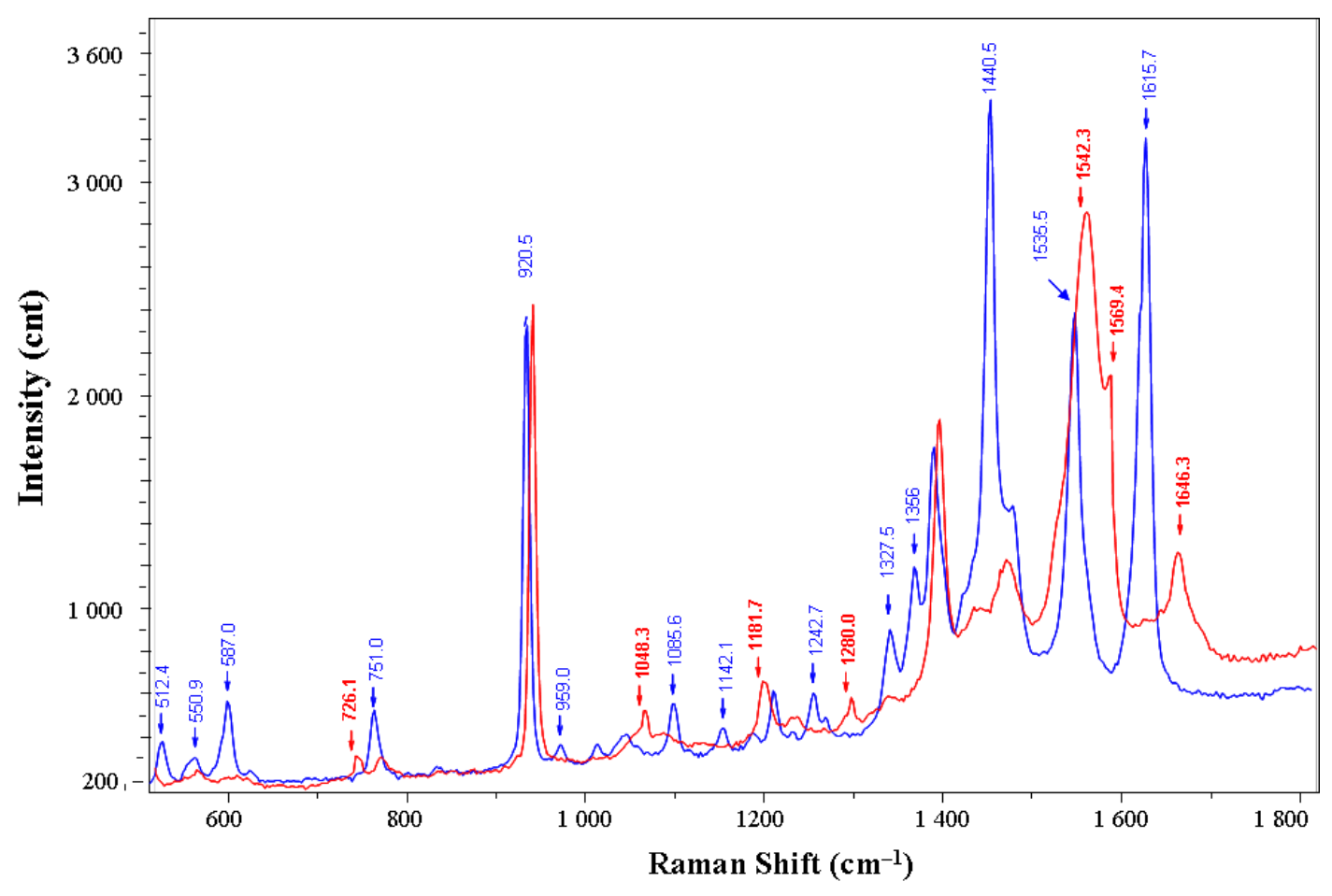

Figure 3.13. Reesonance Raman $(\mathrm{rR})$ spectrum of $\mathbf{3}$ (blue), and $(\mathbf{3})^{2+}(\mathrm{red})$ at $230 \mathrm{~K}\left(\lambda_{\mathrm{ex}}=457 \mathrm{~nm}\right)$.

\subsection{Spectroscopy in the Solid states}

In order to gain further insight into the electronic structure of the cluster cores, all the compounds were characterized by temperature dependent magnetic susceptibility measurements, and in addition compound $\mathbf{5}$ was characterized by zero-field Mössbauer spectroscopy.

3.5.1. Magnetic Properties: Magnetic susceptibility data were collected for $\mathbf{3 - 5}$ in the temperature range from 295 to $2.0 \mathrm{~K}$ in order to characterize the exchange coupling within the triazine core. No significant field dependence was observed when data were measured at applied fields of 0.2 and $0.5 \mathrm{~T}$. The temperature dependence of the molar magnetic susceptibility $\chi_{\mathrm{M}}$ and of the product $\chi_{\mathrm{M}} T$ is shown in Figure 3.14.

The observed $\chi_{\mathrm{M}} T$ value at room temperature is $0.76 \mathrm{~cm}^{3} \mathrm{Kmol}^{-1}$ (corresponding to an effective moment $\mu_{\mathrm{eff}}=2.47 \mu_{\mathrm{B}}$ ) for $3,11.78 \mathrm{~cm}^{3} \mathrm{Kmol}^{-1}$ (corresponding to an effective moment $\mu_{\mathrm{eff}}=9.71 \mu_{\mathrm{B}}$ ) for 4 , and $15.35 \mathrm{~cm}^{3} \mathrm{Kmol}^{-1}$ (corresponding to an effective moment $\mu_{\mathrm{eff}}$ $\left.=11.08 \mu_{\mathrm{B}}\right)$ for $\mathbf{5}$, those are close to the theoretical value expected for two uncoupled copper(II) ions $\left(0.77 \mathrm{~cm}^{3} \mathrm{Kmol}^{-1}\right.$ or $\mu_{\mathrm{eff}}=2.49 \mu_{\mathrm{B}}$ for $\left.g=2.03\right)$ for 3 , for four uncoupled $\mathrm{Mn}^{\text {III }}$ ions $\left(11.89 \mathrm{~cm}^{3} \mathrm{Kmol}^{-1}\right.$ or $\mu_{\text {eff }}=9.75 \mu_{\mathrm{B}}$ for $\left.g=1.99\right)$ for 4 , and four uncoupled Fe ${ }^{\mathrm{III}}$ ions 
$\left(19.56 \mathrm{~cm}^{3} \mathrm{Kmol}^{-1}\right.$ or $\mu_{\mathrm{eff}}=12.52 \mu_{\mathrm{B}}$ for $\left.g=2.12\right)$ for $\mathbf{5}$. Upon lowering the temperature, in the case of $3 \chi_{\mathrm{M}}$ goes through a broad maximum at $55 \mathrm{~K}$ while $\chi_{\mathrm{M}} T$ drops and gradually tends to zero, which is indicative of significant antiferromagnetic coupling between the two copper(II) ions and an $S_{\mathrm{T}}=0$ ground state. For complex $4, \chi_{\mathrm{M}} T$ remains almost constant over a wide temperature range and decreases more rapidly only at low temperatures, suggesting that antiferromagnetic coupling is much weaker in the grid complex. The $\chi_{\mathrm{M}} T$ value of $\mathbf{5}$ decreases gradually upon lowering the tempertaure, from $15.35 \mathrm{~cm}^{3} \mathrm{Kmol}^{-1}\left(11.08 \mu_{\mathrm{B}}\right)$ at 295 $\mathrm{K}$ to $9.15 \mathrm{~cm}^{3} \mathrm{Kmol}^{-1}\left(8.56 \mu_{\mathrm{B}}\right)$ at $25 \mathrm{~K}$. Below $25 \mathrm{~K} \chi_{\mathrm{M}} T$ decreases more rapidly to reach 2.62 $\mathrm{cm}^{3} \mathrm{Kmol}^{-1}\left(4.58 \mu_{\mathrm{B}}\right)$ at $2 \mathrm{~K}$, indicating that dominant antiferromagnetic coupling within the tetranuclear entities.

Experimental data were simulated using a fitting procedure to the appropriate HeisenbergDirac-van-Vleck (HDvV) spin Hamiltonian for isotropic exchange coupling and Zeeman splitting (eq. 3.1) for complex 3, (eq. 3.2; assuming equal $J$ values for all four metal-metal bridges) for complex $\mathbf{4}$, and (eq. 3.3; consider two different coupling constant $J_{1}$, and $J_{2}$ ) for complex 5.

$$
\begin{aligned}
& \hat{H}=-2 J \hat{S}_{1} \cdot \hat{S}_{2}+g \mu_{B}\left(\hat{S}_{1}+\hat{S}_{2}\right) B \\
& \hat{H}=-2 J \sum \hat{S}_{i} \hat{S}_{j}+g \mu_{B} B \sum_{i=1}^{4} \hat{S}_{z i}+\sum_{i=1}^{4} D_{i}\left[S_{z i}^{2}-1 / 3 S_{i}\left(S_{i}+1\right)\right] \\
& \hat{H}=-2 J_{1}\left(\hat{S}_{1} \hat{S}_{2}+\hat{S}_{3} \hat{S}_{4}\right)-2 J_{2} \hat{S}_{2} \hat{S}_{3}+g \mu_{B} B \sum_{i=1}^{4} \hat{S}_{z i}
\end{aligned}
$$

A Curie-Weiss-behaved paramagnetic impurity $(\rho)$ that presumably causes the increase of $\chi_{\mathrm{M}}$ at very low temperatures and temperature-independent paramagnetism (TIP) were included according to $\chi_{\text {calc }}=(1-P I) \cdot \chi+P I \cdot \chi_{\text {mono }}+T I P$. Table 3.3 summarizes the magnetic parameters obtained from best fit analyses. 

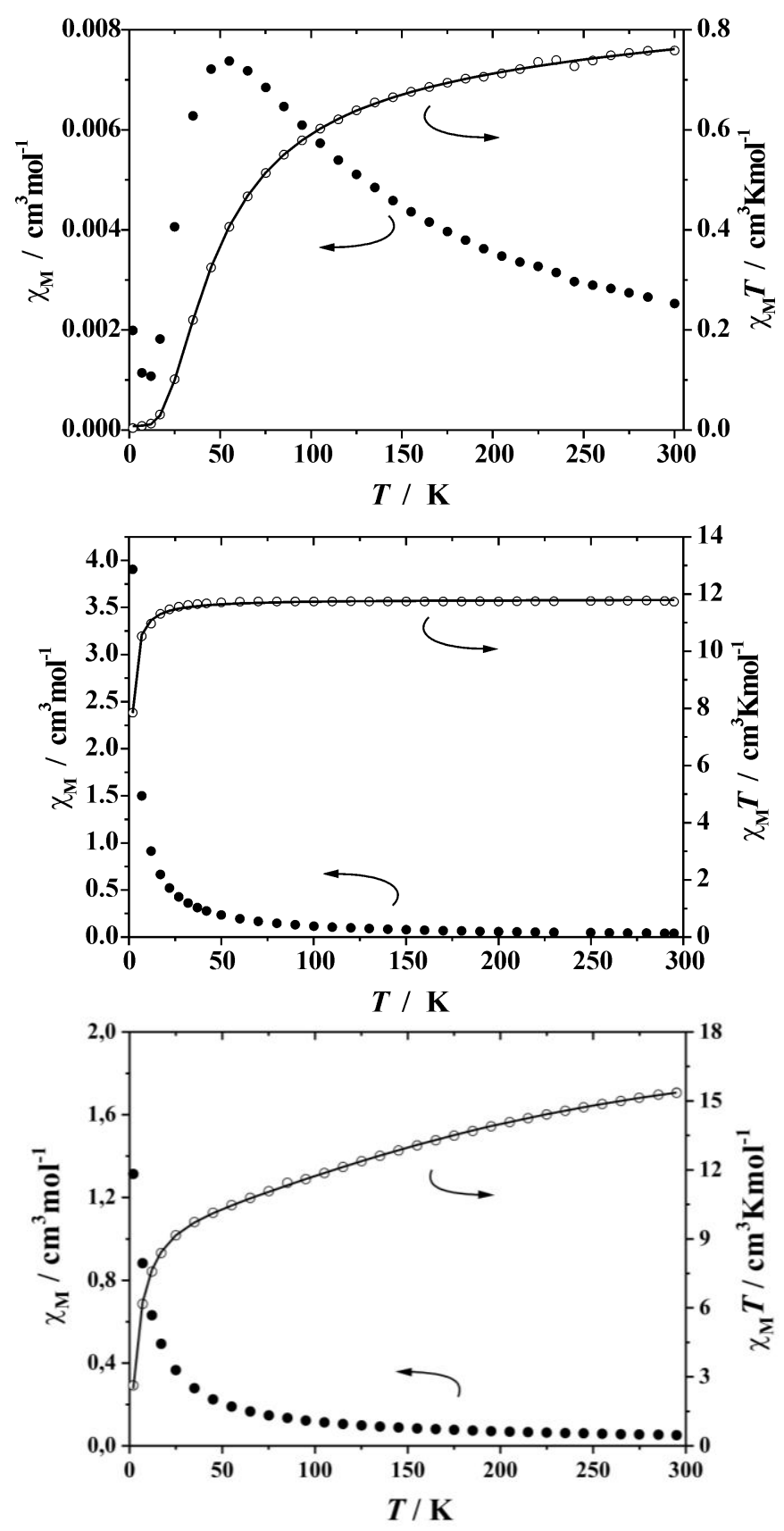

Figure 3.14. Plots of $\chi_{\mathrm{M}}$ (solid circles) and $\chi_{\mathrm{M}} T$ (open circles) versus temperature for $\mathbf{3}$ (top), 4 (middle), and 5 (bottom) at $0.5 \mathrm{~T}$. The solid lines represent the simulated curves.

Table 3.3. Best Fit Parameters of Magnetic Data Analysis for Complexes 3-5.

\begin{tabular}{|l|l|l|l|l|l|}
\hline compound & $g$ & $J\left[\mathrm{~cm}^{-1}\right]$ & $D \mid\left[\mathrm{cm}^{-1}\right]$ & $\rho[\%]$ & $T I P\left[10^{-6} \mathrm{~cm}^{3} \mathrm{~mol}^{-1}\right]$ \\
\hline $\mathbf{3}$ & 2.03 & -30 & - & 1.9 & 1.7 \\
\hline $\mathbf{4}$ & 1.99 & -0.07 & 0.3 & 1.0 & 0 \\
\hline $\mathbf{5}$ & 2.12 & -1.00 and -14.7 & - & 0.7 & 834.2 \\
\hline
\end{tabular}


In agreement with the qualitative evaluation of the $\chi_{\mathrm{M}}$ curves, very weak antiferromagnetic coupling occurs in the $\mathrm{Mn}^{\mathrm{III}}$ system, while antiferromagnetc coupling is significantly stronger in case of $\mathrm{Cu}^{\mathrm{II}}$, despite very similar intramolecular distances between adjacent metal ions $(\sim 6.0 \AA)$. At first sight the antiferromagnetic interaction seems to contradict with the anticipated spin-polarization mechanism that would result from the meta-phenylene type arrangement of the two metal ions. Close inspection of the molecular structure of $\mathbf{3}$ shows that the magnetic $d_{x 2-y 2}$ orbitals of the square-pyramidal $\mathrm{Cu}^{\mathrm{II}}$ ions are located in the equatorial plane, which is roughly parallel to the plane of the triazine ring. The strong d $\sigma-n \sigma$ orbital overlap between the $\mathrm{d}_{\mathrm{x} 2-\mathrm{y} 2}$ orbitals of $\mathrm{Cu} 1$ and $\mathrm{Cu} 2$ and the triazine-N1 and -N2 atoms appears to cause a pronounced spin delocalization effect in the $\mathrm{Cu}-\mathrm{N}$ bonds, which thus provides an efficient antiferromagnetic superexchange pathway through the triazine bridge. Similar antiferromagnetic coupling via $\sigma$-bond superexchange was found also in some pyrimidinebridged dinuclear copper(II)-complexes. ${ }^{45}$

In case of the $\mathrm{Mn}^{\mathrm{III}}$ compound 4 both $\mathrm{d} \sigma$ and $\mathrm{d} \pi$ spins are involved. One should thus expect $\pi$ and $\sigma$-pathways to be operative, with ferro- and antiferromagnetic contributions through $\pi$ spin polarisation and $\sigma$-spin superexchange mediated through the triazine core. Apparently antiferromagnetic contributions prevail, leading to weak overall antiferromagnetic coupling within the triazine core and an $S_{\mathrm{T}}=0$ ground state.

In case of the $\mathrm{Fe}^{\mathrm{III}}$ compound $\mathbf{5}$, weak antiferromagnetic coupling $\left(J_{2}=-1.00 \mathrm{~cm}^{-1}\right)$ is also found through the triazine core due to the above mentioned reason. In addition to that a relatively strong antiferromagntic exchnage interaction $\left(J_{2}=-14.7 \mathrm{~cm}^{-1}\right)$ occurs through oxo bridge, as has previously been observed for many other binuclear oxo-bridged $\mathrm{Fe}^{\mathrm{III}}$ complexes. $^{46}$

3.5.2. Mössbauer Spectroscopy: Mössbauer spectra of compound $\mathbf{5}$ have been recorded at $295 \mathrm{~K}$, and $7 \mathrm{~K}$. The spectrum is shown in Figure 3.15, and the spectral fits to the data are obtained by using Lorentzian line doublets with isomer shifts $\delta$ and quadrupole splitting $\Delta E_{\mathrm{Q}}$ as summarized in Table 3.4. At $295 \mathrm{~K}$ the spectrum exhibits a quadrupole-split doublets appearing as two very broad and asymmetric absorptions. As could be expected from the partly split absorptions it was not possible to fit the spectra at $295 \mathrm{~K}$ by considering a unique quadrupole-split doublet. In order to satisfactorily fit the spectra, it was necessary to consider two nested quadrupole-split doublets, i.e. of very close isomer shift $(\delta)$, in line with a very similar $\mathrm{N}_{2} \mathrm{O}_{4}$ environment for all four $\mathrm{Fe}$ sites, but significantly different quadrupole splittings $\left(\Delta E_{\mathrm{Q}}\right)$. 

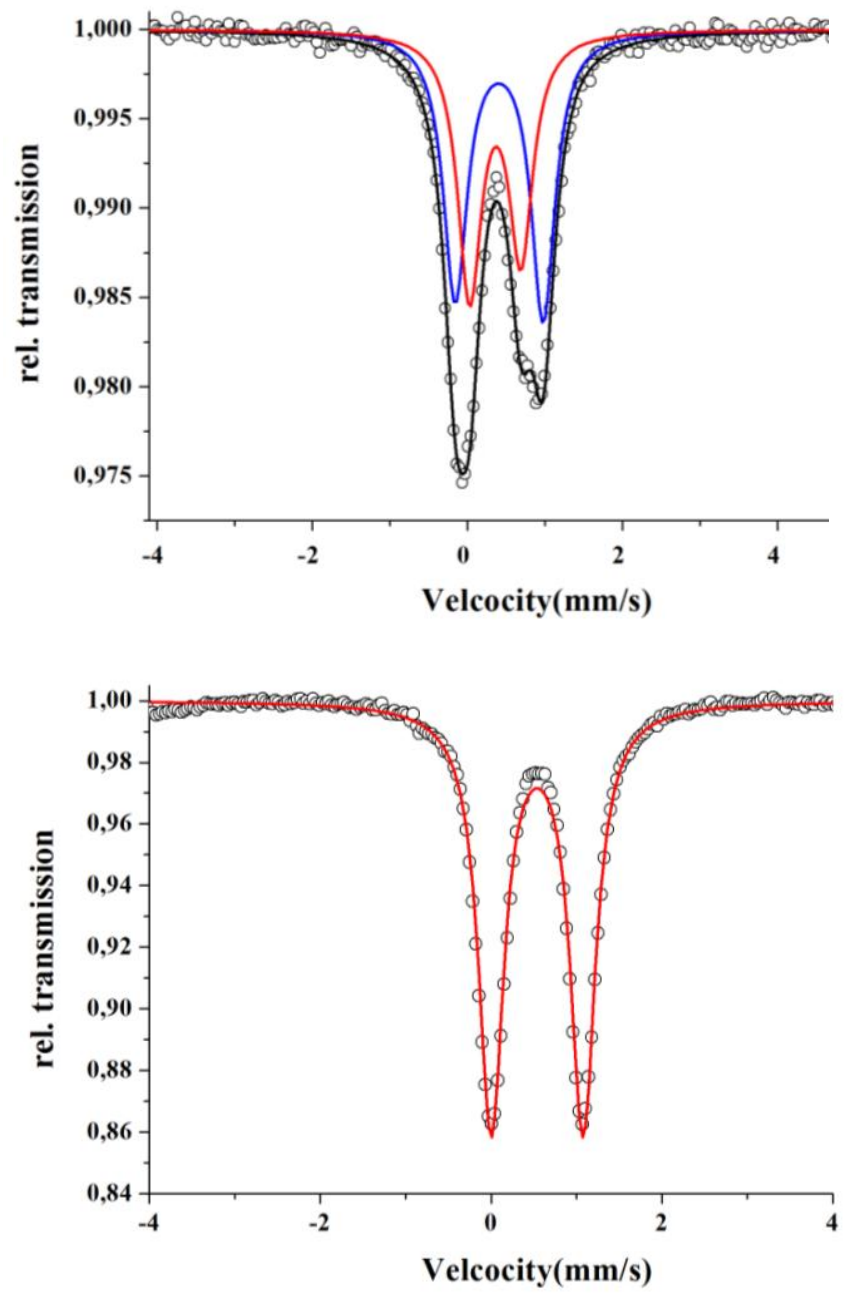

Figure 3.15. Mössbauer spectra of 5 recorded in zero field at 295 (top), and 7 K (bottom). Blue and red spectra (top) are fitted to the experimental values (circles), and the red solid line (bottom) is fitted to the experimental values (circles).

Table.3.4. Mössbauer spectral data for 5 .

\begin{tabular}{|l|l|l|l|l|}
\hline$T(\mathrm{~K})$ & $\delta\left(\mathrm{nm} \mathrm{s}^{-1}\right)$ & $\Delta E_{\mathrm{Q}}\left(\mathrm{nm} \mathrm{s}^{-1}\right)$ & $\Gamma_{1 / 2}\left[\mathrm{~mm} \mathrm{~s}^{-1}\right]$ & Area ratio $(\%)$ \\
\hline 295 & 0.41 & 1.13 & 0.37 & 53 \\
& 0.36 & 0.66 & 0.37 & 46 \\
\hline 7 & 0.54 & 1.08 & 0.36 & 100 \\
\hline
\end{tabular}

For ligand environment and symmetry reasons discussed in the crystal structure section, the ferric sites may be associated by two $\mathrm{Fe}(1)$ and $\mathrm{Fe}\left(1^{\prime}\right)$ on one hand and $\mathrm{Fe}(2)$ and $\mathrm{Fe}\left(2^{\prime}\right)$ on the other hand. Consequently, the simulated data obtained was satisfactory with the area ratio of each doublet to $\sim 50 \%$. However at $7 \mathrm{~K}$, the spectrum exhibits one doublet only with isomer 
shift $(\delta)$ 0.54, and quadrupole splitting $\left(\Delta E_{\mathrm{Q}}\right)$ 1.08, (see Table 3.4). The isomer values are in accord with the $d^{5}$ h.s. nature of the ferric centers, ${ }^{47}$ and the value for the quadrupole splitting is remarkably large in view of the fact that the $d^{5}$ configuration has no valence contribution of the electric field gradient; presumably this reflects the ionic nature bonds to the iron-ligands.

\subsection{Conclusions}

In this chapter we have described the multistep syntheses of a new family of triazine based compartmental ligands, in which to confirm the integrity of triazine core one of the ligand $\mathbf{H}_{3} \mathbf{L}^{\mathbf{5}}$ and its intermediate $\mathbf{1}$ were corroborated by X-ray diffraction analysis. We were able to isolate unusal binuclear $\mathrm{Cu}^{\mathrm{II}}$, tetranuclear $\mathrm{Mn}^{\mathrm{III}}$, and $\mathrm{Fe}^{\mathrm{III}}$ as a metal assisted hydrolysed product. Redox properties of binuclear copper(II) and tetranuclear grid complex of manganese(III) reveal that both complexes possess significant redox stability towards oxidation. Moreover, spectroelectro chemistry, EPR spectroscopy, and rR-spectroscopy suggests that in both cases metal based oxidation occurs and formed high-valent metalphenolate species, that may be fruitful in exploring the devolepment of efficient oxidation catalyst. 


\section{Chapter 4}

\section{Triazine Based Mononuclear, Dinuclear, and 1-D Chain Complexes of Manganese (II) with Some Auxiliary Ligands: Synthesis, Structures, and Magnetic Properties}

\subsection{Introduction}

In recent years, the synthesis and characterization of new low-dimensional materials (one or two-dimensional) that may show ferromagnetic long-range cooperative phenomena (molecular magnets) ${ }^{48}$ have been the main focus of interest in the molecular magnetism area. At present, the field of molecular magnetism is addressing the synthesis and study of discrete polynuclear molecules in an attempt to improve our understanding of the mechanism involved in magnetic coupling ${ }^{49}$ and the production of new paramagnetic clusters. ${ }^{50}$

Among the most used short bridging ligands such as oxalate, cyanide, dicyanamide, and azide $\left(\mathrm{N}_{3}{ }^{-}\right)$, the latter has proved to be very versatile by displaying remarkable diversities in both crystal engineering and magnetism. ${ }^{51}$ This particular property is due to the fact that azide can link metal ions in $\mu$-1,3(end-to-end, EE), $\mu$-1,1(end-on, EO), $\mu$ - $(1,1,1), \mu-(1,1,3), \mu-(1,1,1,1)$, and $\mu-(1,1,3,3)$ or other modes resulting in numerous materials of low dimensionalities. ${ }^{52,48}$ The magnetic exchange mediated via an azido bridge can be ferro- or antiferromagnetic, depending on the bridging mode and bonding parameters. It has been widely stated that the exchange is generally ferromagnetic in nature for the EO mode and antiferromagnetic for the EE mode, although an increasing number of exceptions have been reported recently. ${ }^{51,53}$ The structures and thus the magnetic properties of metal azido systems are obviously sensitive to the coligands employed. To date, various coligands have been introduced into the metal-azido system, giving rise to an abundant range of polymeric complexes showing a wide variety of magnetic properties. ${ }^{54}$, 48 Among the first-row transition-metal azides, the exchange interaction between the high-spin $\mathrm{Mn}^{\mathrm{II}}$ centres is desirable because it contains the highest possible number of unpaired electrons. So, here we prepared novel 2,4,6-Tris[ $\left\{N\right.$-methyl- $N^{\prime}-$ (pyridine-2-yl)methylidene\}hydrazino]-1,3,5-triazine as a co-ligand and observe the behaviour of this particular ligand over Mn-azide system. The synthesis, structural characterisation, and magnetic properties of new triazine based manganese (II) compounds with sodium azide, and sodium dicyanamide as auxiliary ligands are described in detail in this chapter. 


\subsection{Synthesis and Structural Characterisation of Metal Complexes}

Compound $\mathbf{L}^{7}$ was obtained as a white powder in $94 \%$ yield by condensation of 2 pyrdinecarboxaldehyde with 2,4,6-Tris( $N$-methylhydrazino)-1,3,5-triazine in methanol, that was already discussed in previous chapter. The reaction of ligand $\mathbf{L}^{7}$ with 1 equivalent of $\mathrm{Mn}\left(\mathrm{NO}_{3}\right)_{2} \cdot \mathrm{xH}_{2} \mathrm{O}$ in methanol at room temperature mononuclear compound $\mathbf{6}$ was isolated. However in presence of excess metal salt with 1 equivalent of sodium azide interestingly azide bridged dinuclear compound 7 was isolated (Scheme 4.1). In both cases, nitrate and methanol molecule are occupied in the axial position of the metal ion in the crystal structure, those labile group can be replaced by similar or different kind of auxiliary bridging ligands (such as sodium azide, hydrogen cyanamide, dicyanamide etc.) and may be possible to make a symmetric or an asymmetric 1-D chain kind of compound. Therefore those mononuclear and dinuclear compounds 6 and 7 can act as good precursor for making polymeric structures. However when the compound $\mathbf{6}$ was treated with excess sodium azide in methanol at room temperature only mononuclear compound $\mathbf{8}$ was isolated rather than expected product. It was also obtained with ligand, metal salt, and sodium azide molar ratio in 1:1:2 in methanol at room temperature.

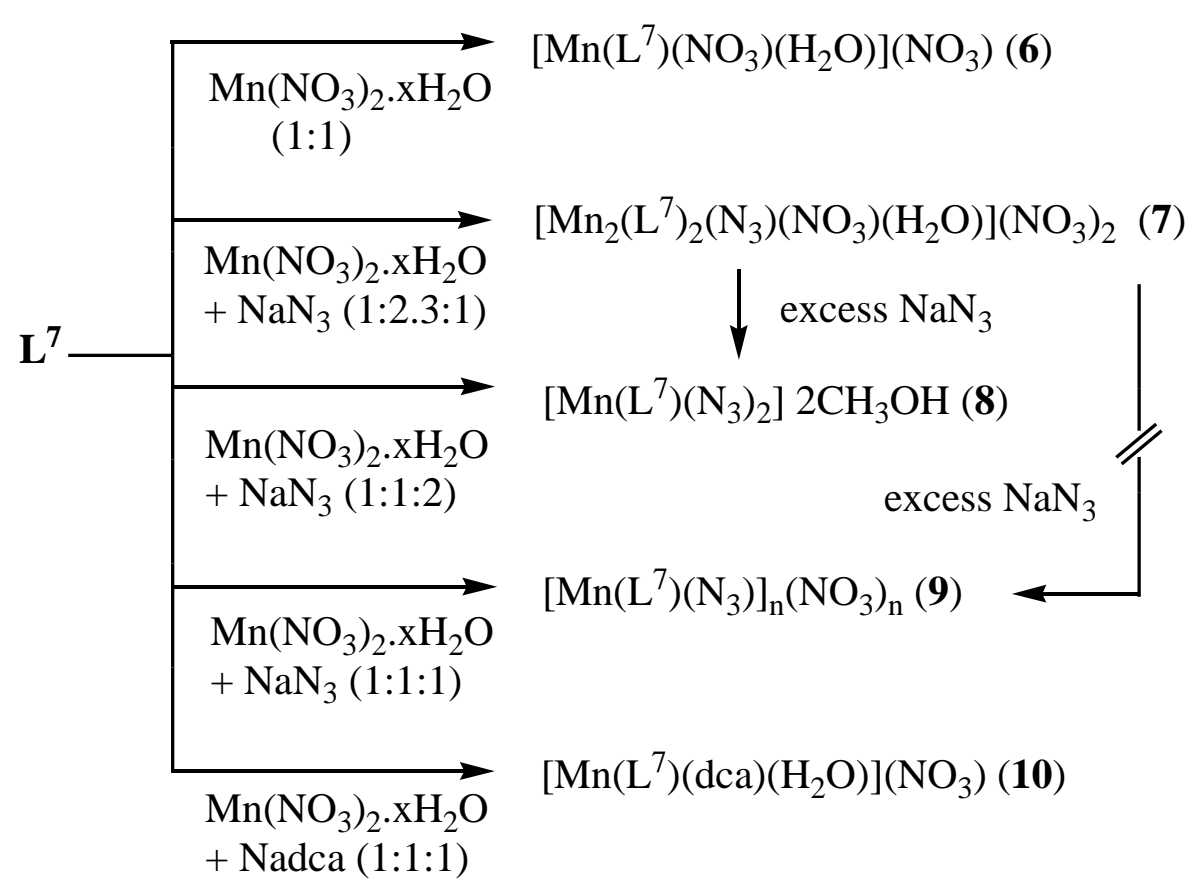

Scheme 4.1. Synthetic scheme of complex 6-10.

Surprisingly with ligand, metal salts, and sodium azide molar ratio in 1:1:1 in methanol at room temperature, $\mu-1,3$ (end to end) azide bridged 1-D polymeric compound 9 was isolated 
(Scheme 4.1). However in same molar ratio at reflux condition a mixture of mononuclear compound 8, and chain compound $\mathbf{9}$ were isolated. Thus it suggests that nature of selfassembly highly depend upon the reaction condition as well as stoichiometry of the ligand, metal, and sodium azide molar ratio. Using same stoichiometry with sodium dicyanamide in methanol at room temperature, only mononuclear dicyanamide complex $\mathbf{1 0}$ was isolated, it may be due to bent nature of the dicyanamide inhabit to formation of chain compound.

Molecular structure of compound $\mathbf{6}$ and $\mathbf{8}$ are depicted in Figure 4.1. In both cases, Mn(II) ion having unusual hepta coordinated pincer-like binding motif, in where two $\mathrm{N}$ sites from py, two $\mathrm{sp}^{2}$ hydrazone $\mathrm{N}$ sites and $\mathrm{N} 1$ site of the central triazine ring coordinate to the metal ion in equatorial position and axial position are occupied by nitrate and water molecule (for compound 6) and two azide ions (for compound $\mathbf{8}$ ).
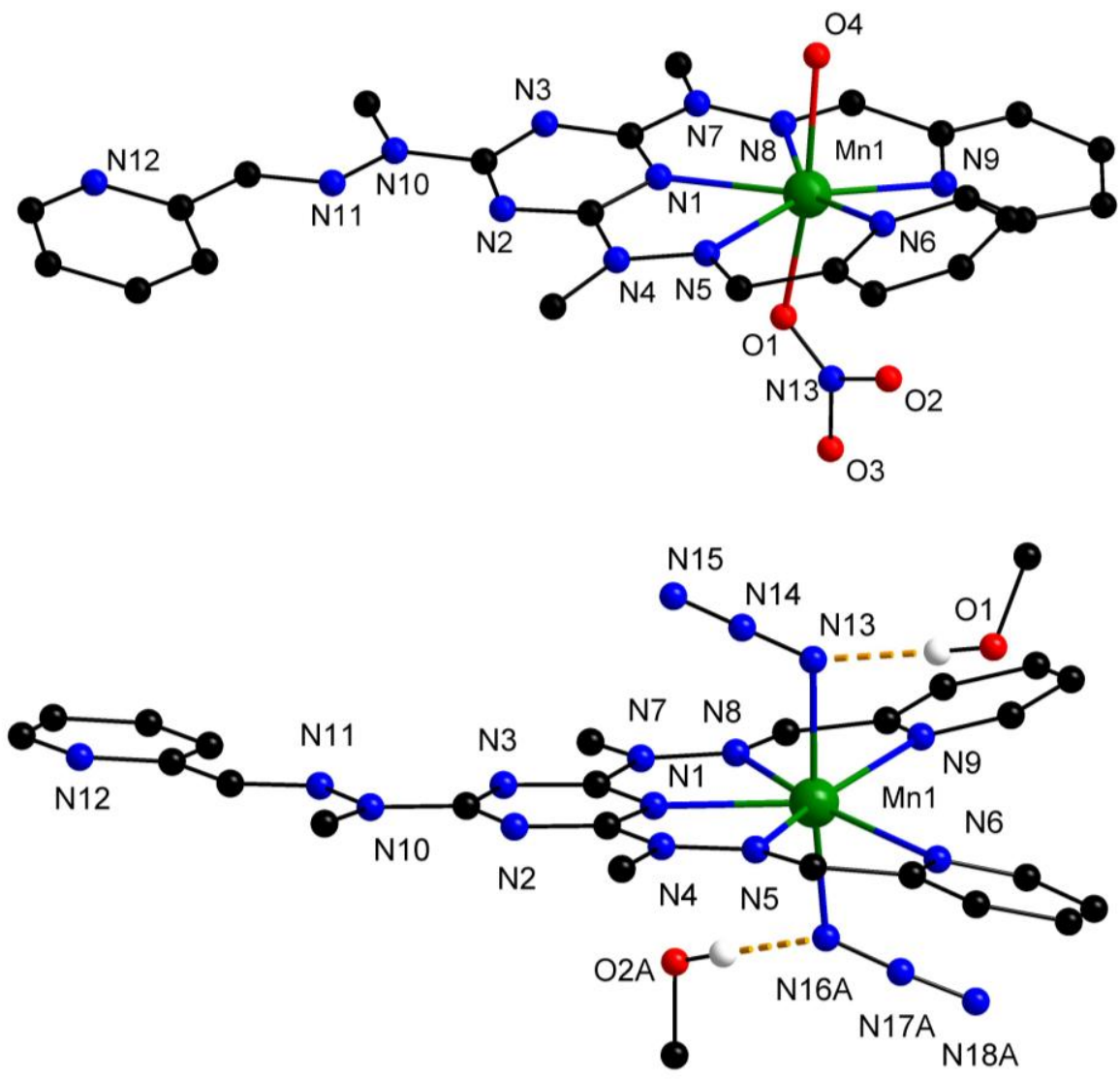

Figure 4.1. View of the molecular structure of $\mathbf{6}$ (top) and 8 (bottom). In the interests of clarity all hydrogen atoms have been omitted. Selected atom distances (Å) for 6: Mn1-N1 2.27(2), Mn1-N8 2.37(2), Mn1-N6 2.34(2), Mn1-O4 2.16(2), Mn1-O1 2.15(2), H10-H17 2.31(2), Selected bond angles $\left(^{\circ}\right)$ for 6: N1-Mn1-N8 65.94(4), N1-Mn1-N5 66.32(3), N5-Mn1-N6 68.99(3), N8-Mn1-N9 68.95(4), N6-Mn1-93.11(3), O1-Mn1-O4 166.1(2), and Selected atom distances ( $\mathrm{A})$ for 8: Mn1-N1 2.28(2), Mn1-N8 2.38(2), Mn1-N6 2.41(2), Mn1-N13 2.21(1), Mn1-N16A 2.18(1), H10-H17 2.17(1), Selected bond angles $\left(^{\circ}\right)$ for 8: N1-Mn1-N8 65.50(2), N1-Mn1N5 66.18(3), N5-Mn1-N6 68.02(2), N8-Mn1-N9 68.54(3), N6-Mn1-93.34(2), N131-Mn1-N16A 173.49(4). 
Such kind of pincer-like binding motif of the metal ion enforces to make a twisted conformation of the two py rings in equatorial plane to minimize the steric interaction. The bond lengths between two twisted pyridine rings $\mathrm{H}$ atoms (at $6^{\text {th }}$ position of the pyridine) are $2.31 \AA$ for 6 and $2.17 \AA$ for 8 . The dihedral angles between the two planes N1-N5-N6-Mn1, and N1-N8-N9-Mn1 are $13.16^{\circ}$ and $9.7^{\circ}$ for $\mathbf{6}$, and $\mathbf{8}$ respectively. Methanol solvent molecule is H-bonding with two axial azide ions in compound $\mathbf{8}$, which may diminish the twisted conformation.

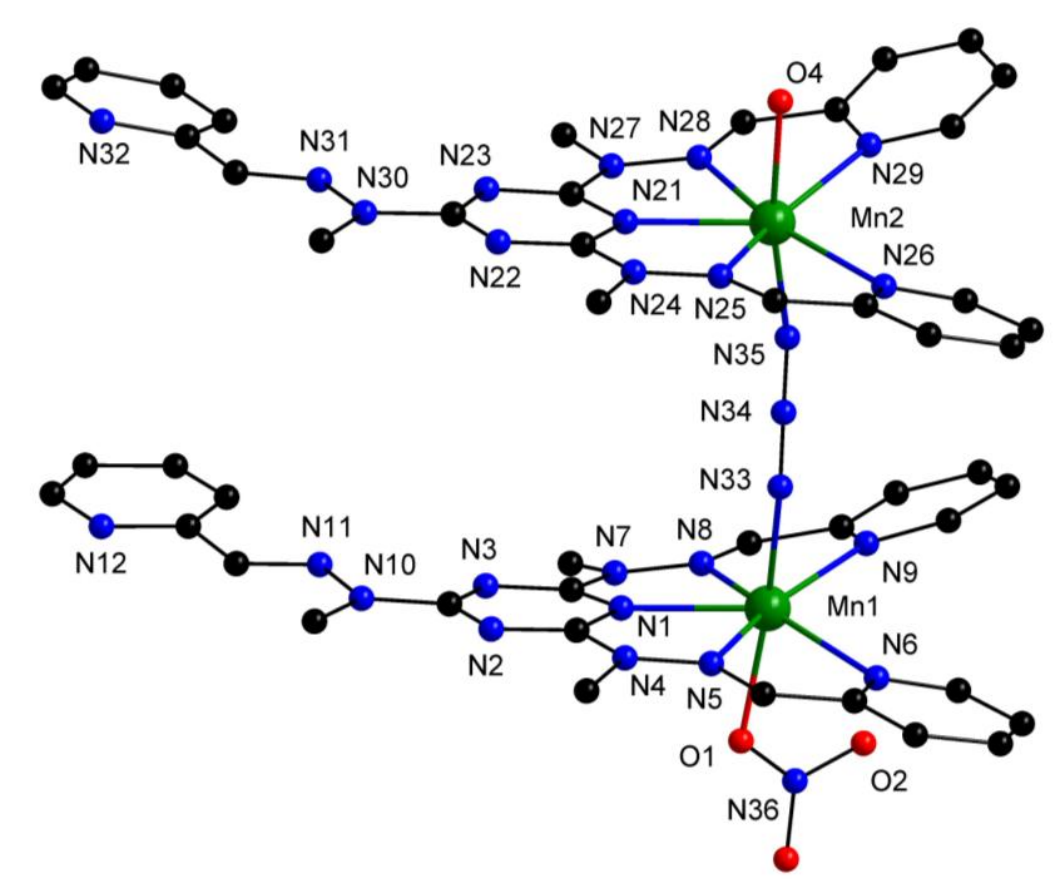

Figure 4.2. View of the molecular structure of 7. In the interests of clarity all hydrogen atoms have been omitted. Selected atom distances (Å) for 7: Mn1-N1 2.27(2), Mn2-N21 2.28(2), Mn1-N9 2.32(2), Mn2-N29 2.38(2), Mn1-N9 2.33(2), Mn2-N29 2.37(2), Mn1-N33 2.22(2), Mn2-N35 2.19(2), Mn1-O1 2.23(2), Mn2-O4 2.18(2), H10-H17 2.31(2), H40-H47 2.21 (2). Selected bond angles $\left(^{\circ}\right)$ for 7: Mn1-N33-N34 155.1(8), Mn2N35-N34 149.9(8), Mn1-N33-N35-Mn2 156.9(15), N9-Mn1-N6 93.2(6), N26-Mn2-N29 94.0(6).

Molecular structure of compound 7 (space group $P-1$ ) is shown in Figure 4.2. Compound 7 features a distorted pentagonal bipyramidal geometry at the heptacoodinated metal ions where the equatorial position of the metal ions are occupied by two $\mathrm{N}$-sites from py, two $\mathrm{sp}^{2}$ hydrazones $\mathrm{N}$-sites and $\mathrm{N}$-site of the triazine ring as like before, and axial position are bridged by $\mu-1,3$ (end to end) azide binding mode form a binuclear complex. The structural parameters of the equatorial position is quite similar as like complexes $\mathbf{6}$ and $\mathbf{8}$, and only differences is the coordination sphere of metal ions in axial position. Two different bond angle of Mn1-N33-N34, and Mn2-N35-N34 in compound 7 are $155.1^{\circ}, 149.9^{\circ}$ respectively 
implies that asymmetric $\mu-1,3$ binding mode occurs of the azide ion. The dihedral angle between the mean planes Mn1-N33-N34-N35 and Mn2-N35-N34-N33 $(\tau)$ is $22.93^{\circ}$.

The 1D chain structure of compound 9 (space group $P-1$ ) as determined by X-Ray crystallography is depicted in Figure 4.3, and details of their selected bond length and bond angles are shown in Figure 4.4.

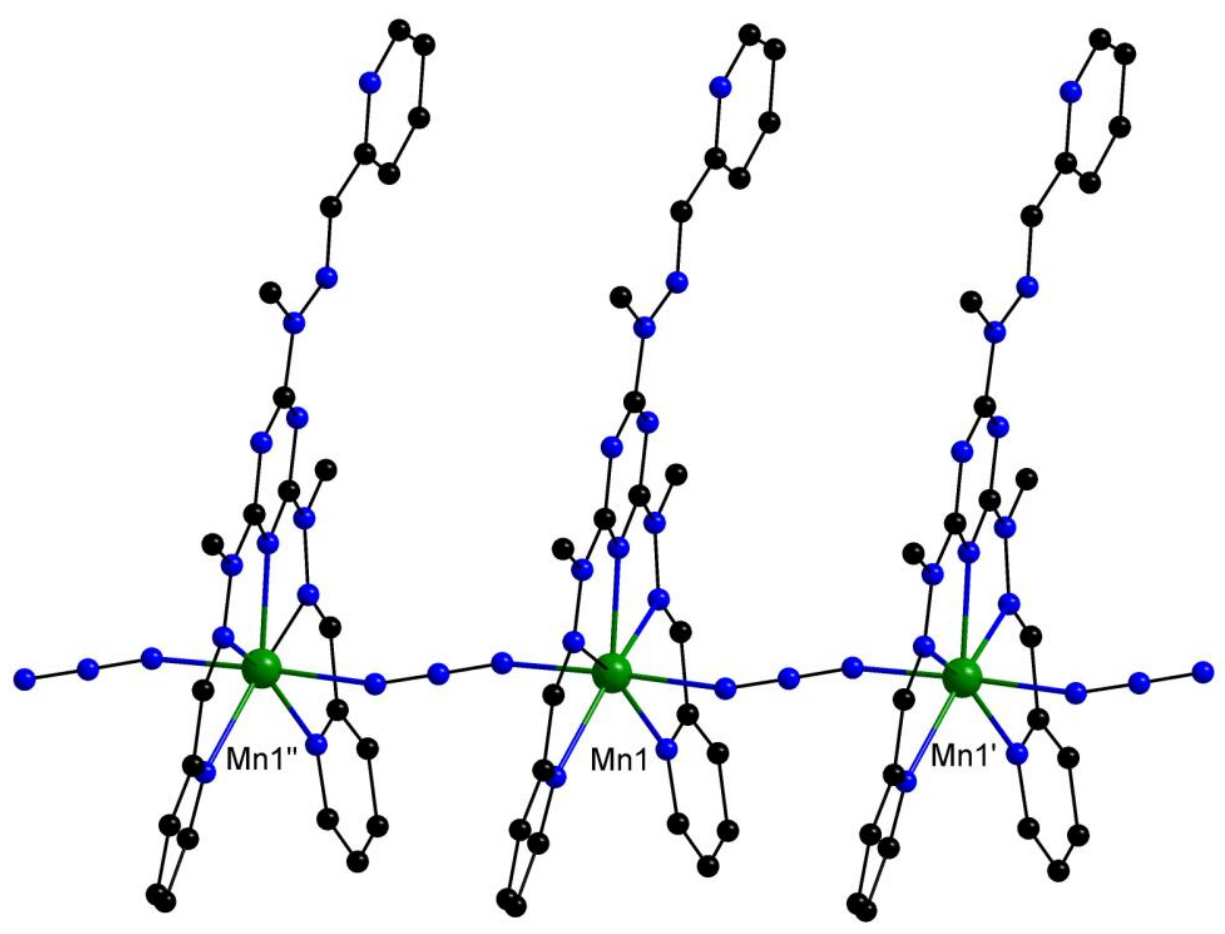

Figure 4.3 View of the molecular structure of 9 . In the interests of clarity all hydrogen atoms have been omitted.

Here the equatorial position of the $\mathrm{Mn}$ (II) ion is occupied by $\mathrm{N}$ sites of py, hydrazone, and triazine ring like before in pincer-binding motif, but axial position linked by $\mu-1,3$-azido ligands to give a anticipated extended 1D structure. The structural parameters (Figure 4.3) of the metal ion are quite similar with the dinuclear complex 7, except the bond angle between the azide linkages. The bond angle of Mn1-N15-N14 and Mn1 -N13-N14 are $158.9^{\circ}$ and $159.01^{\circ}$ respectively, and the standard deviation between the angle of Mn1-N15-N14 and Mn1'-N13-N14 is $~ 0.08$ which implies that symmetric $\mu-1,3$ binding mode occurs in azide. The dihedral angle between the mean planes Mn1-N15-N14-N13 and Mn1 -N13-N14-N15 $(\tau)$ is $9.54^{\circ}$. It should be mentioned that those angles are major factor to determine the magnetic properties of compound $\mathbf{7}$, and $\mathbf{9}$. 


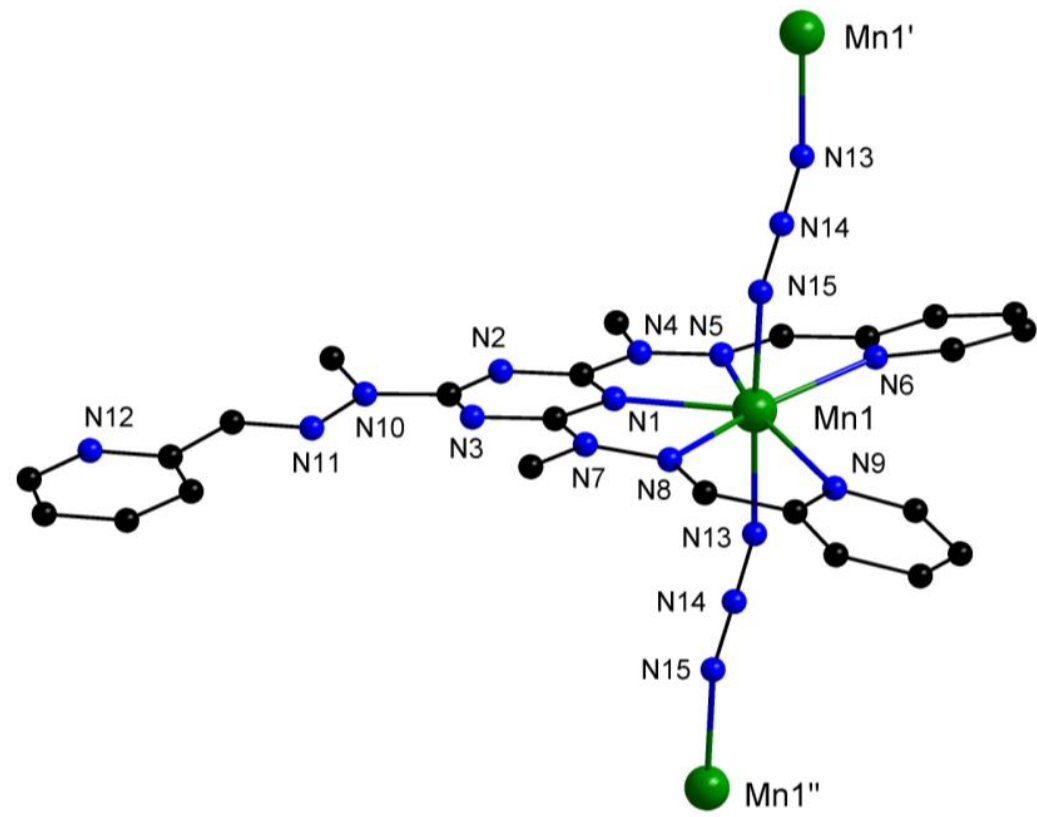

Figure 4.4. View of the molecular structure of 9. In the interests of clarity all hydrogen atoms have been omitted. Selected atom distances (A) for 9: Mn1-N1 2.27(2), Mn1-N5 2.34(2), Mn1-N6 2.34(2), Mn1-N13 2.26(1), Mn1-N15 2.21(1), Mn1'-N13 2.26(1), Mn1“"-N15 2.21(1), H10-H17 2.22(1). Selected bond angles ( ${ }^{\circ}$ ) for 9: N6-Mn1-N9 92.6(5), N5-Mn1-N6 69.1(5), N8-Mn1-N9 68.6(5), N1-Mn1-N5 66.4(9), Mn1-N15-N13 159(6), Mn1'-N13-N15 158.9(6), Mn1-N13-N15 158.9(6), Mn1“-N15-N13 159(6).

Infrared spectroscopy is a useful tool to elucidate the details of azide coordination, bond positions in the azide stretching region exhibit relatively minor variations for the systems investigated here. Compound 8, and 9 show strong adsorptions at 2037, and $2054 \mathrm{~cm}^{-1}$ respectively, while compound 7 show two dominant bands at $2108 \mathrm{~cm}^{-1}$ and $2061 \mathrm{~cm}^{-1}$ charecteristic for asymmetric $\mu-1,3$ binding mode.

Molecular structure of compound $\mathbf{1 0}$ is depicted in Figure 4.5. It crystallises in triclinic space group $P-1$. The coordination environment of the $\mathrm{Mn}^{\mathrm{II}}$ ion is similar as like mononuclear complex 6, difference are only mainly expressed in the axial position of the metal ion, in where dicyanamide as abridging ligand instead of nitrate. 


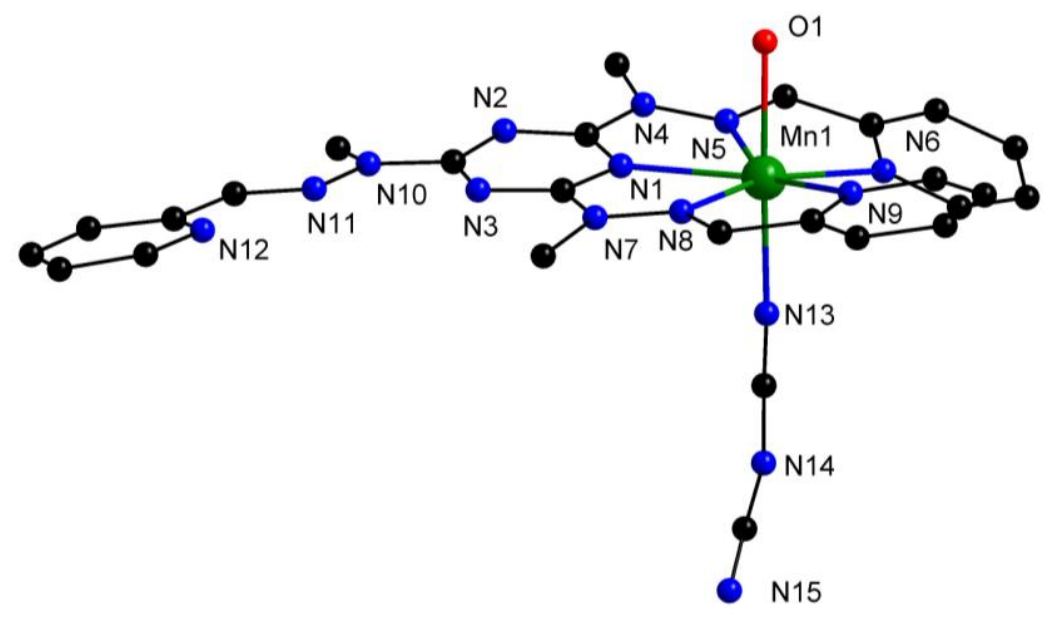

Figure 4.5. View of the molecular structure of 10. In the interests of clarity all hydrogen atoms have been omitted. Selected atom distances $(\AA ̊ ㇒)$ for 10: Mn1-N1 2.27(2), Mn1-N8 2.37(2), Mn1-N6 2.34(2), Mn1-O4 2.16(2), Mn1-O1 2.15(2), H10-H17 2.31(2), and Selected bond angles $\left(^{\circ}\right)$ for 10: N1-Mn1-N8 65.94(4), N1Mn1-N5 66.32(3), N5-Mn1-N6 68.99(3), N8-Mn1-N9 68.95(4), N6-Mn1-93.11(3), O1-Mn1-O4 166.1(2).

\subsection{Magnetic Properties}

In order to gain further insight into the electronic structure of all the compounds temperature dependent magnetic susceptibility measurements was performed.

Magnetic susceptibility data were collected for all the complexes in the temperature range from 295 to $2.0 \mathrm{~K}$. No significant field dependence was observed when data were measured at applied fields of 0.2 and $0.5 \mathrm{~T}$. The temperature dependence of the molar magnetic susceptibility $\chi_{\mathrm{M}}$ and of the product $\chi_{\mathrm{M}} T$ is shown in Figure 4.6. The observed $\chi_{\mathrm{M}} T$ value at $295 \mathrm{~K}$ is $4.3 \mathrm{~cm}^{3} \mathrm{Kmol}^{-1}$ (corresponding to an effective moment $\mu_{\text {eff }}=5.84 \mu_{\mathrm{B}}$ ) for $\mathbf{6}, 8.15$ $\mathrm{cm}^{3} \mathrm{Kmol}^{-1}$ (corresponding to an effective moment $\mu_{\mathrm{eff}}=8.08 \mu_{\mathrm{B}}$ ) for $7,4.6 \mathrm{~cm}^{3} \mathrm{Kmol}^{-1}$ (corresponding to an effective moment $\mu_{\text {eff }}=6.1 \mu_{\mathrm{B}}$ ) for $\mathbf{8}, 4.20 \mathrm{~cm}^{3} \mathrm{Kmol}^{-1}$ (corresponding to an effective moment $\mu_{\mathrm{eff}}=5.8 \mu_{B}$ ) for 9 , and $4.2 \mathrm{~cm}^{3} \mathrm{Kmol}^{-1}$ (corresponding to an effective moment $\left.\mu_{\text {eff }}=5.8 \mu_{\mathrm{B}}\right)$ for $\mathbf{1 0}$. These are close to the theoretical value expected for one manganese(II) ion $\left(4.2 \mathrm{~cm}^{3} \mathrm{Kmol}^{-1}\right.$ or $\mu_{\mathrm{eff}}=5.8 \mu_{\mathrm{B}}$ for $\left.g=1.96\right)$ for $\mathbf{6},\left(4.6 \mathrm{~cm}^{3} \mathrm{Kmol}^{-1}\right.$ or $\mu_{\mathrm{eff}}=$ $6.06 \mu_{\mathrm{B}}$ for $\left.g=2.05\right)$ for $\mathbf{8},\left(4.45 \mathrm{~cm}^{3} \mathrm{Kmol}^{-1}\right.$ or $\mu_{\mathrm{eff}}=5.97 \mu_{\mathrm{B}}$ for $\left.g=2.02\right)$ for $\mathbf{9}$, and $(4.12$ $\mathrm{cm}^{3} \mathrm{Kmol}^{-1}$ or $\mu_{\mathrm{eff}}=5.75 \mu_{\mathrm{B}}$ for $g=1.94$ ) for $\mathbf{1 0}$, and for two uncoupled manganese(II) ions $\left(8.5 \mathrm{~cm}^{3} \mathrm{Kmol}^{-1}\right.$ or $\mu_{\mathrm{eff}}=8.23 \mu_{\mathrm{B}}$ for $\left.g=1.97\right)$ for $\mathbf{7}$. In case of the compound $\mathbf{6}, \mathbf{8}$, and $\mathbf{1 0}$, $\chi_{\mathrm{M}} T$ value remains constant with decreasing the temperature, thus indicating there is no any significant intermolecular magnetic interaction of the manganese ion and those are nicely 


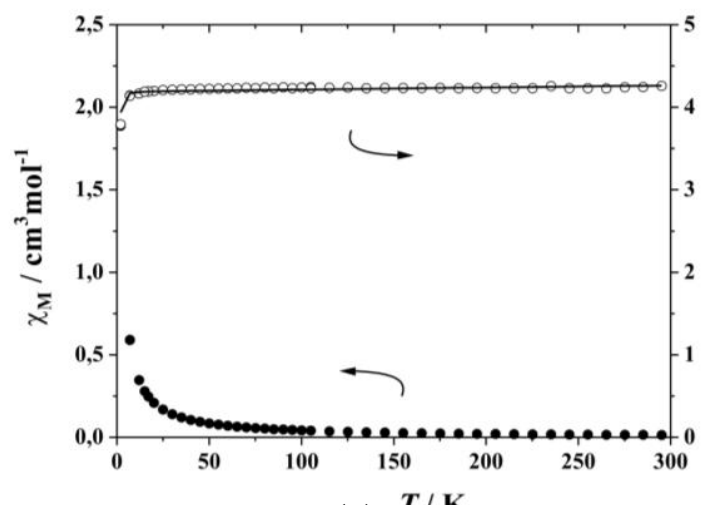

(a) $T / \mathbf{K}$

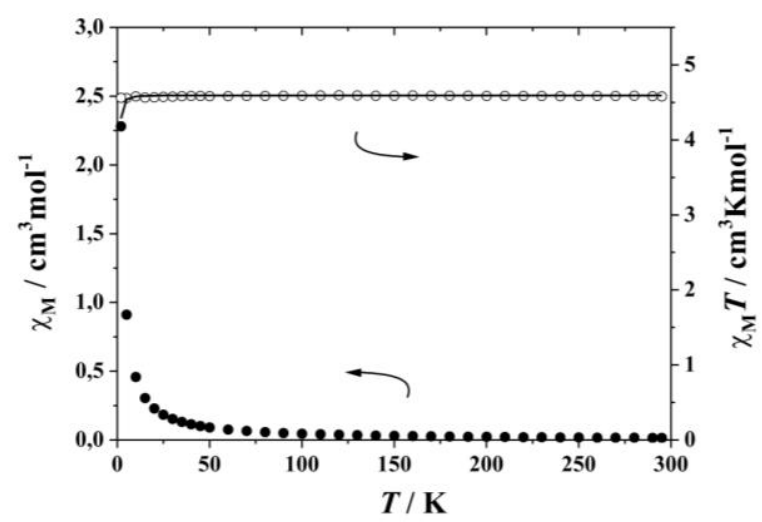

(c)

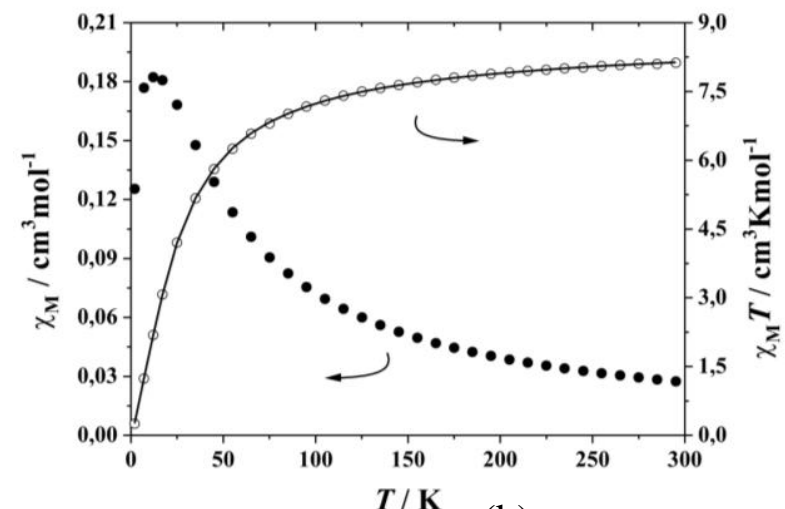

(b)

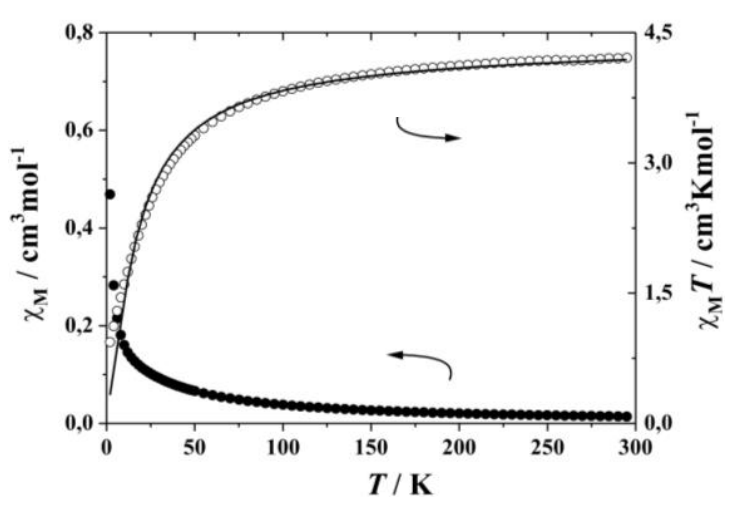

(d)

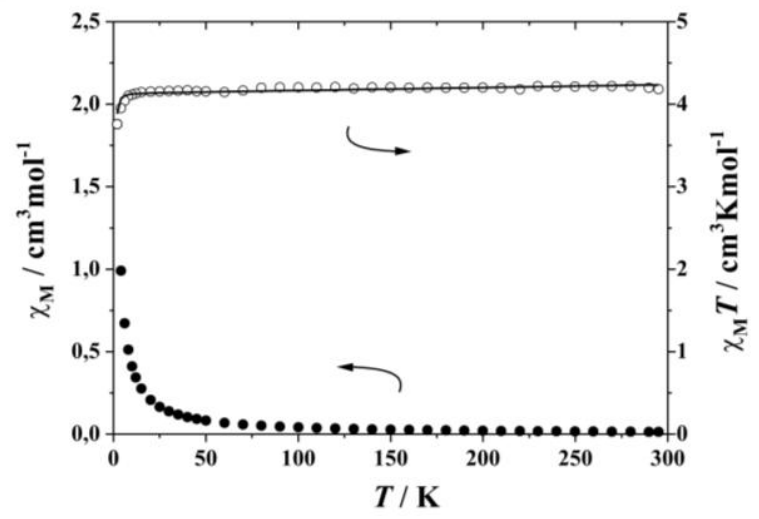

(e)

Figure 4.6. Plots of $\chi_{M}$ (solid circles) and $\chi_{M} T$ (open circles) versus temperature for $\mathbf{6}$ (a), 7 (b), 8 (c), and 10 (c) at $0.5 \mathrm{~T}$ and $9(\mathrm{~d})$ at $0.2 \mathrm{~T}$. The solid black lines represent the simulated curves.

obey the curie law, and at low temperature, $\chi_{M} T$ value slowly decreases, that may be due to saturation. For compound $\mathbf{7}$ and $\mathbf{9}$ upon lowering the temperature, $\chi_{\mathrm{M}} T$ value gradually decreases, which is in accordance with overall antiferromagnetic coupling and an $S=0$ ground state. Experimental data were simulated using a fitting procedure to the appropriate Heisenberg-Dirac-van-Vleck (HDvV) spin Hamiltonian for isotropic exchange coupling and Zeeman splitting (eq. 4.1) for 7 and $\mathbf{9}$. 


$$
\hat{H}=-2 J \hat{S}_{1} \cdot \hat{S}_{2}+g \mu_{B}\left(\hat{S}_{1}+\hat{S}_{2}\right) B
$$

Table 4.2 summarizes the magnetic parameters obtained from best-fit analyses. The results indicate that there exists an intramolecular antiferromagnetic spin exchange interaction between the two $\mathrm{Mn}^{\mathrm{II}}$ ions in both complexes. The magnetic behavior is in good agreement with this fact, where the super-exchange interaction in the $\mathrm{M}-\mathrm{N}-\mathrm{N}-\mathrm{N}-\mathrm{M}$ mode is antiferromagnetically coupled. It is also in accordance with those of $\mu-1,3$-azide-bridged $\mathrm{Mn}^{\mathrm{II}}$ complexes. ${ }^{51,55}$

Table 4.2. Best Fit Parameters of Magnetic Data Analysis for Complexes 6-10

\begin{tabular}{|l|l|l|l|l|}
\hline compound & $g$ & $J\left[\mathrm{~cm}^{-1}\right]$ & $P I[\%]$ & $T I P\left[10^{-6} \mathrm{~cm}^{3} \mathrm{~mol}^{-1}\right]$ \\
\hline $\mathbf{6}$ & 1.96 & - & 0 & 248.9 \\
\hline $\mathbf{7}$ & 1.97 & -1.75 & 1.5 & 472.3 \\
\hline $\mathbf{8}$ & 2.05 & - & 0 & 0 \\
\hline $\mathbf{9}$ & 2.02 & -1.53 & 5 & 0 \\
\hline $\mathbf{1 0}$ & 1.94 & - & 0 & 353.8 \\
\hline
\end{tabular}

\subsection{Conclusions}

From the above study it can be inferres that for the generation of new magnetic materials with particular triazine as a coligand, the choice of the metal atom and correct auxiliary ligand is very important. Using sodium azide as a complementary ligand, a novel triazine based mononuclear, dinuclear, and 1-D chain complex of $\mathrm{Mn}^{\mathrm{II}}$ were isolated, conflictingly only mononuclear complex was observed in presence of sodium dicyanamide. In the dinuclear and chain structure, azide ion act as a $\mu_{1,3}$ binding mode, and mainly mediates an antiferromagnetic interaction between the $\mathrm{Mn}^{\mathrm{II}}$ ions. Studies of these types of similar compounds with different secondary ligand are in progress, from which it may be able to determine the role of weak interactions over the magnetic behaviour of a molecule. 


\section{Chapter 5}

\section{Pyrazole Based Homometallic Complexes: $\mathrm{M}_{4} \mathrm{O}_{4}\left(\mathrm{M}=\mathrm{Ni}^{\mathrm{II}}\right.$, $\mathrm{Co}^{\mathrm{II}}, \mathrm{Fe}^{\mathrm{II}}$ ) Cubes with High-Spin Ground States}

\subsection{Introduction}

The last two decades have witnessed an upsurge in studies related to magnetism with the aim of understanding the phenomenon of exchange coupling. ${ }^{18}$ Particularly, designed synthesis of multinuclear complexes exhibiting ferromagnetic exchange coupling has attracted the unbeaten interest of inorganic chemists, and deals with twofold challenges: i) synthesis and ii) control of the mechanism of exchange coupling. ${ }^{8}$

When the interacting centers are sufficiently close so that the exchange interaction is mediated via small bridging group like oxo, hydroxo, etc, then the sign of the interaction depends on the relative symmetries of the two magnetic orbitals involved, in accord with the GoodenoughKanamori rules, ${ }^{56}$ and this principle has been exploited in controlling the magnetic properties of polynuclear coordination complexes. ${ }^{18,57}$ When the interacting orbitals are not close together, the interaction is more dependent on the orbitals of the bridging ligand in two mechanistic ways: i) superexchange and ii) spin polarization mechanisms, among which the superexchange mechanism is predominant in metal complexes. The isotropic superexchange interaction is expressed by the spin Hamiltonian, $H=-2 \Sigma J i j S i \cdot S j$, where $J$ is the exchange coupling constant. When two homo-metal ions are bridged by an anion with a bridging angle of $180^{\circ}$, strong antiferromagnetic interactions $(J<0)$ occur. There are two ways to have ferromagnetic interactions. When different metal ions with orthogonal magnetic orbitals are bridged with a bond angle of $180^{\circ}$, ferromagnetic interactions occur, and if two homo-metal ions are bridged with a bond angle of $90^{\circ}$, the magnetic orbitals become orthogonal giving rise to a high-spin ground state.

Transition metal complexes with a cubane structure are a very important class of compounds, which have four metal ions and four anions are alternately aligned in the cube, often have a high-spin ground state ${ }^{58}$ because the magnetic orbitals of the metal ions are accidentally orthogonal. In this regard here I designed new pyrazole based tridentate diol ligand (Scheme 1.5) which can bridges to the four metal ions; build a new cubane core structure. Owing to the flexibility in the coordination geometry around metal centers, these cubanes can show ferroas well as antiferromagnetic exchange interacttions depending on the $\mathrm{M}-\mathrm{O}-\mathrm{M}$ bridging angles and distortion of the $\mathrm{M}_{4} \mathrm{O}_{4}$ cubes. The major aim of modern magneto-chemists is to develop a 
better understanding of the structural and chemical features governing the electron spin-spin interactions in these versatile geometric arrays of four metal ions. Thus ligand synthesis, cluster synthesis, and spectroscopic characterisation of metal cubes composed of nickel (II), cobalt(II), and iron(II) are described in this chapter.

\subsection{Ligand Synthesis}

The new ligand 2-\{3-(2-hydroxyphenyl)-1H-pyrazol-1-yl $\}$ ethanol $\left(\mathbf{H}_{2} \mathbf{L}^{\mathbf{9}}\right)$ has been synthesized in two steps starting from 2-hydroxy acetophenone (Scheme 5.1). The intermediate 1-(2-hydroxyphenyl)-3-dimethylaminoprop-2-enone was obtained according to literature method ${ }^{59}$ and was then heated to reflux with excess $\beta$-hydroxyethyl hydrazine in methanol solution for $2 \mathrm{~h}$. Extraction of the crude product with light petroleum using a soxhlet apparatus gave $\mathbf{H}_{2} \mathbf{L}^{9}$ as needle-shaped white crystals in $45 \%$ yield. The molecular structure of $\mathbf{H}_{2} \mathbf{L}^{9}$ has been determined by X-ray crystallography (Figure 5.1). It forms a propeller-like hydrogen-bonded tetramer via the aliphatic $\mathrm{OH}$ groups, while the phenolic $\mathrm{OH}$ groups are involved in intramolecular hydrogen-bonding to the pyrazole-N.

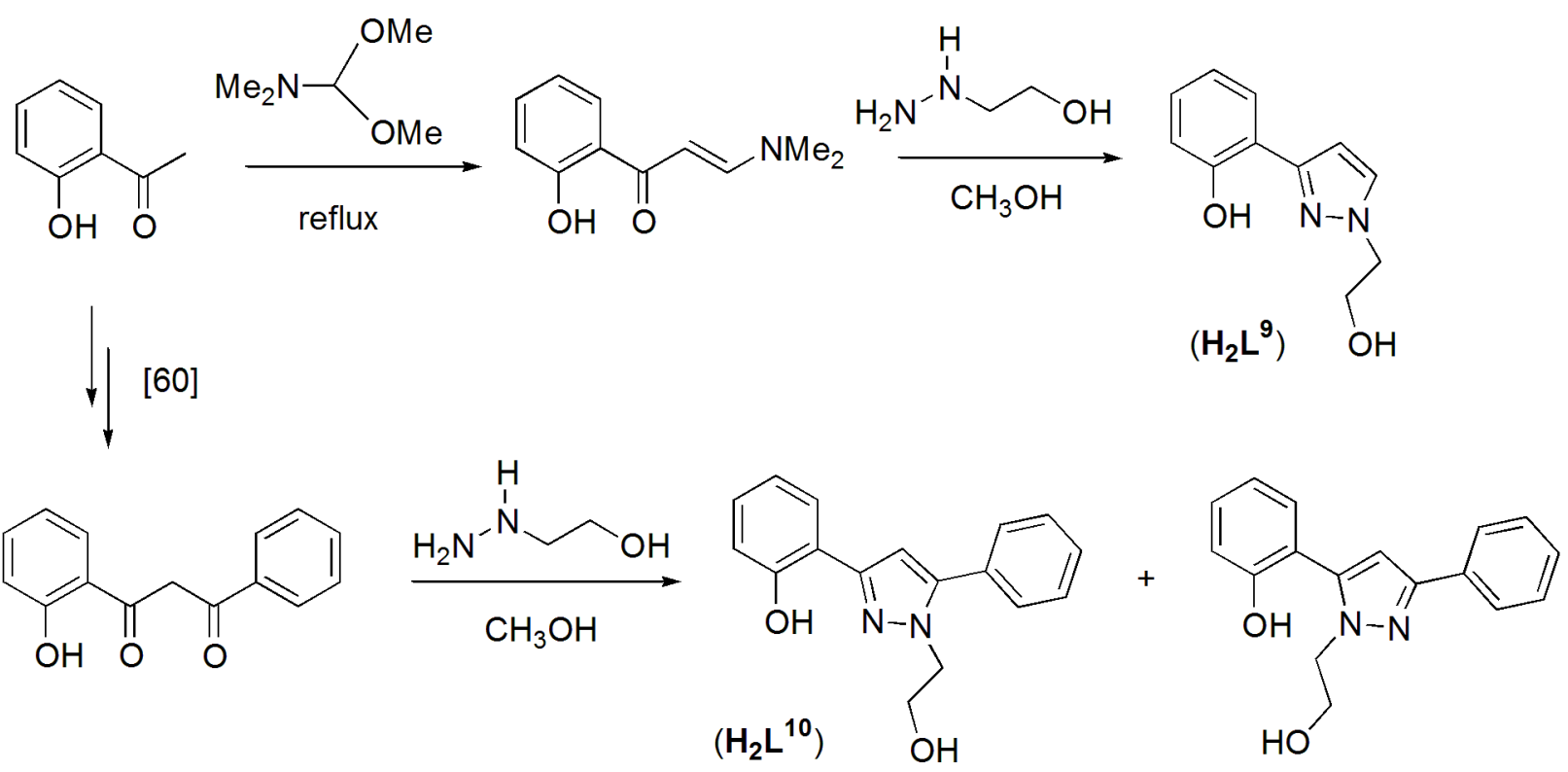

Scheme 5.1. General Synthesis of the New Ligands $\mathbf{H}_{2} \mathbf{L}^{9}$, and $\mathbf{H}_{2} \mathbf{L}^{10}$.

Analogous ligand $\mathbf{H}_{2} \mathbf{L}^{\mathbf{1 0}}$ has been synthesized in multistep process starting from 2-hydroxy acetophenone (Scheme 5.1). The intermediate 1-(2-hydroxyphenyl)-3-phenylpropane-1,3dione was obtained according to literature method ${ }^{60}$ and was then heated to reflux with excess $\beta$-hydroxyethyl hydrazine in methanol solution for $2 \mathrm{~h}$ as like before, and then crude product was extracted with light petroleum using a soxhlet apparatus gave a desired product in $5 \%$ 
yield. Here percentage of yield is relatively poor, that probably due to steric congestion between the bulky phenyl group at C-2 position of the pyrazole, and its neighbour's hydroxyl ethyl group, and prefers other conformation (see Scheme 5.1).

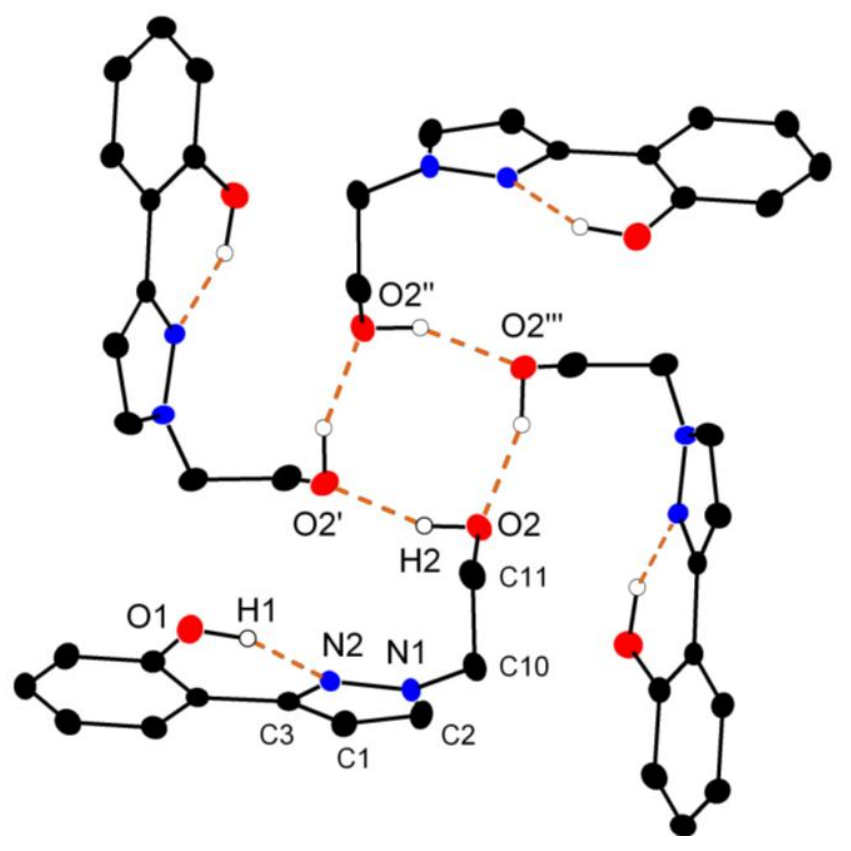

Figure 5.1. View of the molecular structure of $\mathbf{H}_{2} \mathbf{L}$. Selected atom distances ( $\AA$ ) for $\mathbf{H}_{2} \mathbf{L}^{\mathbf{9}}$ : N1-N2 1.3511(22), O1-H1 0.9426(338), O2-H2 0.9426(338), N2-H1 1.7930(385), N2-H2 2.9913(332), N2-C3 1.3427(24), C1-C2 1.3757(28), N1-C2 1.3432(28), N1-C10 1.4607(26), C10-C11 1.5096(32).

Another promising enantiomerically pure ligand $\mathbf{H}_{2} \mathbf{L}^{11}$ was designed by incoporating a chiral centre to the adjacent aliphatic hydroxyl group. It has been synthesized by modifying the previously reported procedure (Scheme 5.2). ${ }^{61}$<smiles>COc1ccccc1-c1cc[nH]n1</smiles>

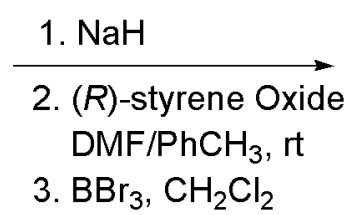<smiles>Oc1ccccc1-c1ccn(C[C@@H](O)c2ccccc2)n1</smiles>
$\left(\mathrm{H}_{2} \mathrm{~L}^{11}\right)$ Scheme 5.2. General synthesis of ligand $\mathbf{H}_{2} \mathbf{L}^{11}$

The reaction of 3-(2-methoxyphenyl) pyrazole ${ }^{62}$ with equimolar amount of sodium hydride in toluene/DMF mixture followed by ring opening reaction of $(R)$-styrene oxide affords a desired 
O-protected ligand, and then deprotection with boron tribromide in $\mathrm{CH}_{2} \mathrm{Cl}_{2}$ gave a desired product in $70 \%$ yield.

Here all the tridentate ligands are a very useful means to build high-nuclearity clusters. In this work, $\mathbf{H}_{2} \mathbf{L}^{9}$ is selected to investigate $3 d$ transition metal complexes, $3 d-3 d$, and $3 d-4 f$ mixed metal clusters.

\subsection{Cluster synthesis and structural characterisation}

Treatment of a methanolic solution of $\mathbf{H}_{2} \mathbf{L}^{9}$ with 1 equiv of $\mathrm{Ni}(\mathrm{OAc})_{2} \cdot 4 \mathrm{H}_{2} \mathrm{O}$ and 2 equiv of $\mathrm{NaOH}$ lead to the rapid precipitation of a dark green solid, that was recrystallised by layering a solution of $\mathrm{CH}_{2} \mathrm{Cl}_{2}$ with $n$-hexane to afford large green crystals of $\left[\mathrm{Ni}_{4}(\mathrm{MeOH})_{4}\left(\mathrm{~L}^{9}\right)_{4}\right](\mathbf{1 1})$. In a similar manner complexes $\left[\mathrm{Co}_{4}(\mathrm{MeOH})_{3}\left(\mathrm{H}_{2} \mathrm{O}\right)\left(\mathrm{L}^{9}\right)_{4}\right](\mathbf{1 2})$, and $\left[\mathrm{Fe}_{4}(\mathrm{MeOH})_{4}\left(\mathrm{~L}^{9}\right)_{4}\right](\mathbf{1 3})$ were obtained using $\mathrm{NEt}_{3}$ as a base and $\mathrm{Co}\left(\mathrm{ClO}_{4}\right)_{2} \cdot 6 \mathrm{H}_{2} \mathrm{O}$, and $\mathrm{FeCl}_{2} \cdot 4 \mathrm{H}_{2} \mathrm{O}$ as metal salt respectively.

Molecular structure of compounds 11-13 are shown in Figure 5.2. A comparison of selected interatomic distances and angles are provided in Table 5.1. Complexes 11, and $\mathbf{1 3}$ crystallize in the tetragonal space group $I 4_{1} / a$ and complex 12 crystallizes in triclinic space group $P-1$. Complexes 11-13 have tetranuclear cubane core structures, in which four $\mathrm{M}^{\mathrm{II}}(\mathrm{M}=\mathrm{Ni}, \mathrm{Co}, \mathrm{Fe})$ ions are bridged by $\mu_{3}$-alkoxo groups, giving an approximately cubane array of alternating metal ions and oxygen atoms. The six coordination sites of the $\mathrm{M}^{\mathrm{II}}$ ions are occupied by four oxygen atoms and one nitrogen atom from a chelating pyrazole based ligand and methanol molecules. The peripheral ligation is completed by four terminal $\mathrm{MeOH}$ ligands for complexes 11, and 13, and three terminal $\mathrm{MeOH}$ and one $\mathrm{H}_{2} \mathrm{O}$ ligands for compound 12, these are participate in intramolecular hydrogen-bonding interaction $(\mathrm{O} \cdots \cdot \mathrm{O}$ distances: $2.59-2.68$ $\AA$ ) with the phenoxo-type $\mathrm{O}$ atoms of the $\left(\mathrm{L}^{9}\right)^{2-}$ ligands, across four of the six faces of the cubane. As a result those four faces exhibit shorter $\mathrm{M} \cdots \cdot \mathrm{M}$ separations, smaller M-O-M (M= $\mathrm{Ni}, \mathrm{Co}, \mathrm{Fe}$ ) angles, and smaller $\mathrm{M}-\mathrm{O}-\mathrm{O}-\mathrm{M}$ dihedral angles (Table 5.1). In addition, all complexes appear to posses a tetragonal elongation of the metal coordination, which occurs along the O-M-O vector involving the methanol and trans alkoxo ligands, with $\mathrm{M}-\mathrm{O}\left(\left(\mathrm{L}^{9}\right)^{2-}\right.$ ethoxo) on this vector of 2.12-2.14 $\AA$ for 11, 2.19-2.25 $\AA$ for 12, and 2.26-2.27 $\AA$ for $\mathbf{1 3}$ in 'axial' versus 2.02-2.06 $\AA$ for 11, 2.04-2.09 $\AA$ for 12, and 2.04-2.13 $\AA$ for $\mathbf{1 3}$ for the "equatorial” $\mathrm{M}-\mathrm{O}\left(\mathrm{L}^{9}\right)^{2-}$-ethoxo) bonds. Thus, all complexes display tetragonal elongation in the metal co-ordination sphere. The bridging angle of the M-O-M bonds, is crucial factor to 
(a)

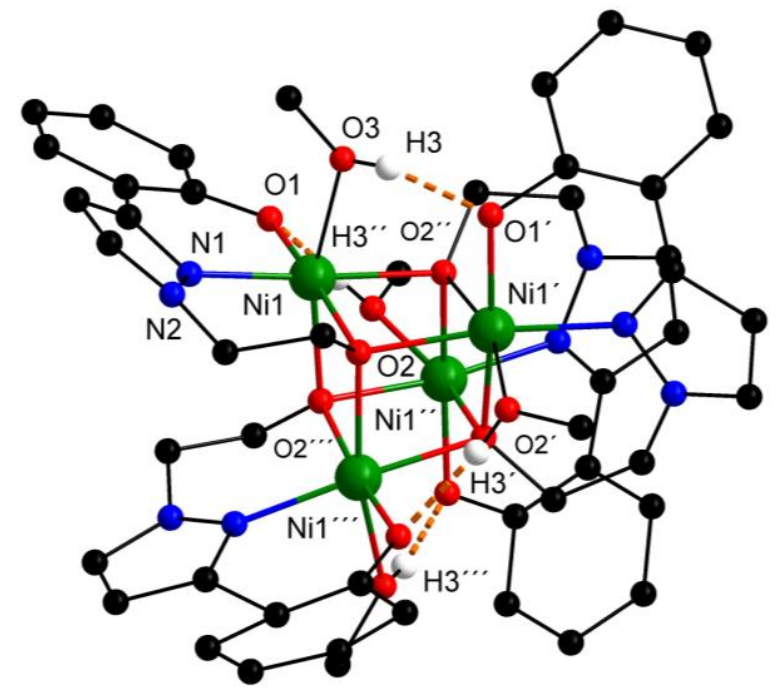

(b)

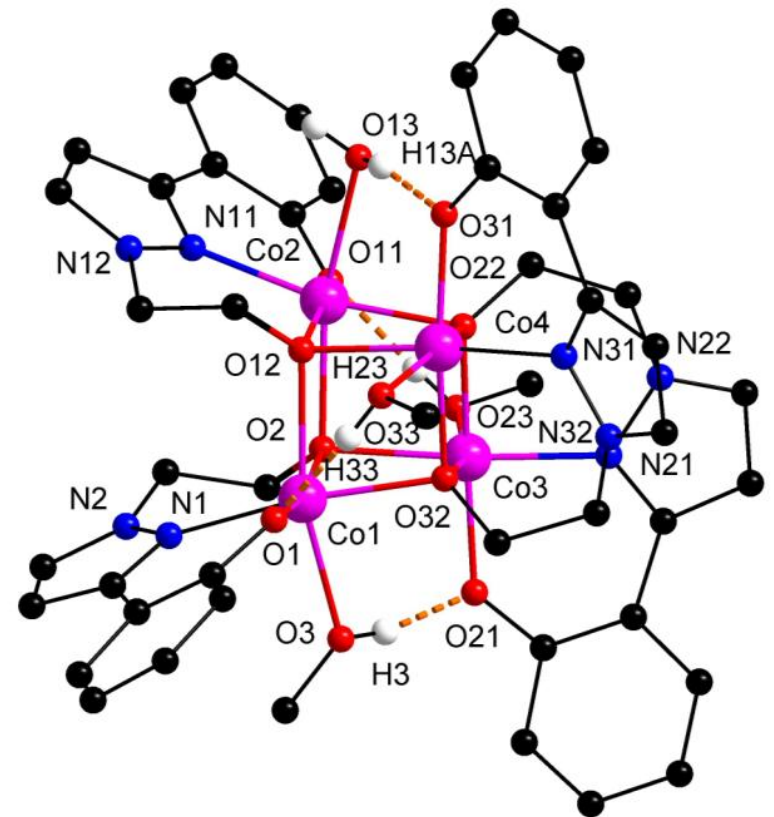

(c)

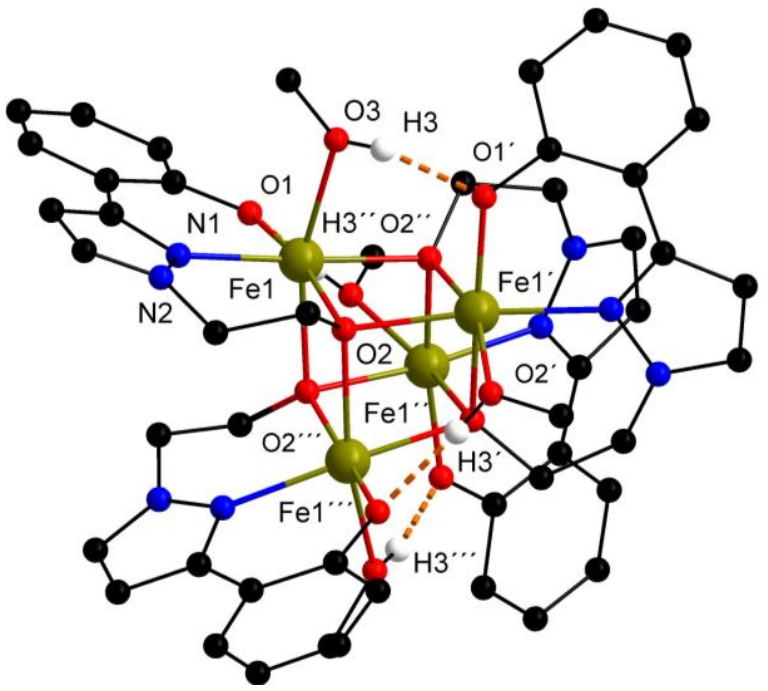

Fiugure 5.2. Molecular structure of $\mathrm{Ni}_{4}(\mathrm{MeOH})_{4}\left(\mathrm{~L}^{9}\right)_{4}(\mathbf{1 1})(\mathrm{a}), \mathrm{Co}_{4}(\mathrm{MeOH})_{3}\left(\mathrm{H}_{2} \mathrm{O}\right)\left(\mathrm{L}^{9}\right)_{4}(\mathbf{1 2})(\mathrm{b})$, and $\mathrm{Fe}_{4}(\mathrm{MeOH})_{4}\left(\mathrm{~L}^{9}\right)_{4}(\mathbf{1 3})(\mathrm{c})$. 
Table 5.1: Selected Interatomic Distances $(\AA)$ and Angles (deg) for 11-13

\begin{tabular}{|l|l|l|l|}
\hline Parameter & $\mathbf{1 1}$ & $\mathbf{1 2}$ & $\mathbf{1 3}$ \\
\hline $\mathrm{M} \cdots \mathrm{M}^{\mathrm{a}}$ & 3.08 & $3.09-3.12$ & 3.16 \\
\hline $\mathrm{M} \cdots \mathrm{M}^{\mathrm{b}}$ & 3.17 & $3.29-3.30$ & 3.32 \\
\hline $\mathrm{M} \cdots \mathrm{O}^{\mathrm{c}}$ & $2.01-2.06$ & $2.03-2.11$ & $2.05-2.13$ \\
\hline $\mathrm{M} \cdots \mathrm{O}^{\mathrm{d}}$ & $2.11-2.14$ & $2.19-2.25$ & $2.26-2.27$ \\
\hline $\mathrm{O} \cdots \mathrm{O}^{\mathrm{e}}$ & 2.64 & $2.59-2.64$ & 2.68 \\
\hline $\mathrm{M}-\mathrm{O}-\mathrm{M}^{\mathrm{a}}$ & $95.07-98.0$ & $92.51-97.87$ & $91.98-98.63$ \\
\hline $\mathrm{M}-\mathrm{O}-\mathrm{M}^{\mathrm{b}}$ & 100.3 & $102.14-102.64$ & 100.56 \\
\hline $\mathrm{M}-\mathrm{O}-\mathrm{O}-\mathrm{M}^{\mathrm{a}}$ & 166 & $163-164$ & 166 \\
\hline $\mathrm{M}-\mathrm{O}-\mathrm{O}-\mathrm{M}^{\mathrm{b}}$ & 175 & $178-180$ & 179 \\
\hline
\end{tabular}

${ }^{a}$ Across the four hydrogen-bonded faces. ${ }^{b}$ Across the two non-hydrogen bonded faces. ${ }^{c}$ "Equatorial" $\left(\mathrm{L}^{9}\right)^{2-}$ ethoxo. ${ }^{\mathrm{d}}$ "Elognated" $\left(\mathrm{L}^{9}\right)^{2-}$-ethoxo. ${ }^{\mathrm{e}} \mathrm{O}-\mathrm{H} \cdots \mathrm{O}$.

determine the sign of the magnetic exchange interactions through oxygen bridges, usually it shows ferromagnetic to antiferromagnetic when the M-O-M bridging angles increase above $98^{\circ}$. It should be mentioned that several $\mathrm{M}_{4}^{\mathrm{II}}\left(\mathrm{M}=\mathrm{Cu}^{2+}-\mathrm{Mn}^{2+}\right)$ complexes with cubane core have been reported previously using schiff base type of tridentate ligand. ${ }^{58}$ Here this is the first time such kind of distorted cubane core was observed with novel pyrazole based ligand scaffold.

\subsection{Spectroscopy in Solution}

In order to clarify the stability of all new complexes in solution, UV/Vis spectroscopy, ESI mass spectrometry, and electrochemical experimets were performed.

5.3.1. Stability of the Cube Complexes in Solution: Positive ion ESI-MS spectrum of dichlomethane solution of compound 11 show molecular ion peak at $\mathrm{m} / \mathrm{z} 1042.9$, and 1064.9 characteristic for single charged ions of $\left[\left(\mathrm{L}^{9}\right)_{4} \mathrm{Ni}_{4}+\mathrm{H}\right]^{+}$and $\left[\left(\mathrm{L}^{9}\right)_{4} \mathrm{Ni}_{4}+\mathrm{Na}\right]^{+}$respectively. It suggests that complex $\mathbf{1 1}$ is stable in solution. Positive ion ESI-MS spectrum of dichlomethane solution of compound 12 also display molecular ion peak at $\mathrm{m} / \mathrm{z} 1045.0$ for $\left[\left(\mathrm{L}^{9}\right)_{4} \mathrm{Co}_{4}+\mathrm{H}\right]^{+}$implies that tetra nuclear cube-type complex stay intact in solution, and the spectrum of complex 11, and $\mathbf{1 2}$ are shown in Figure 5.3. 

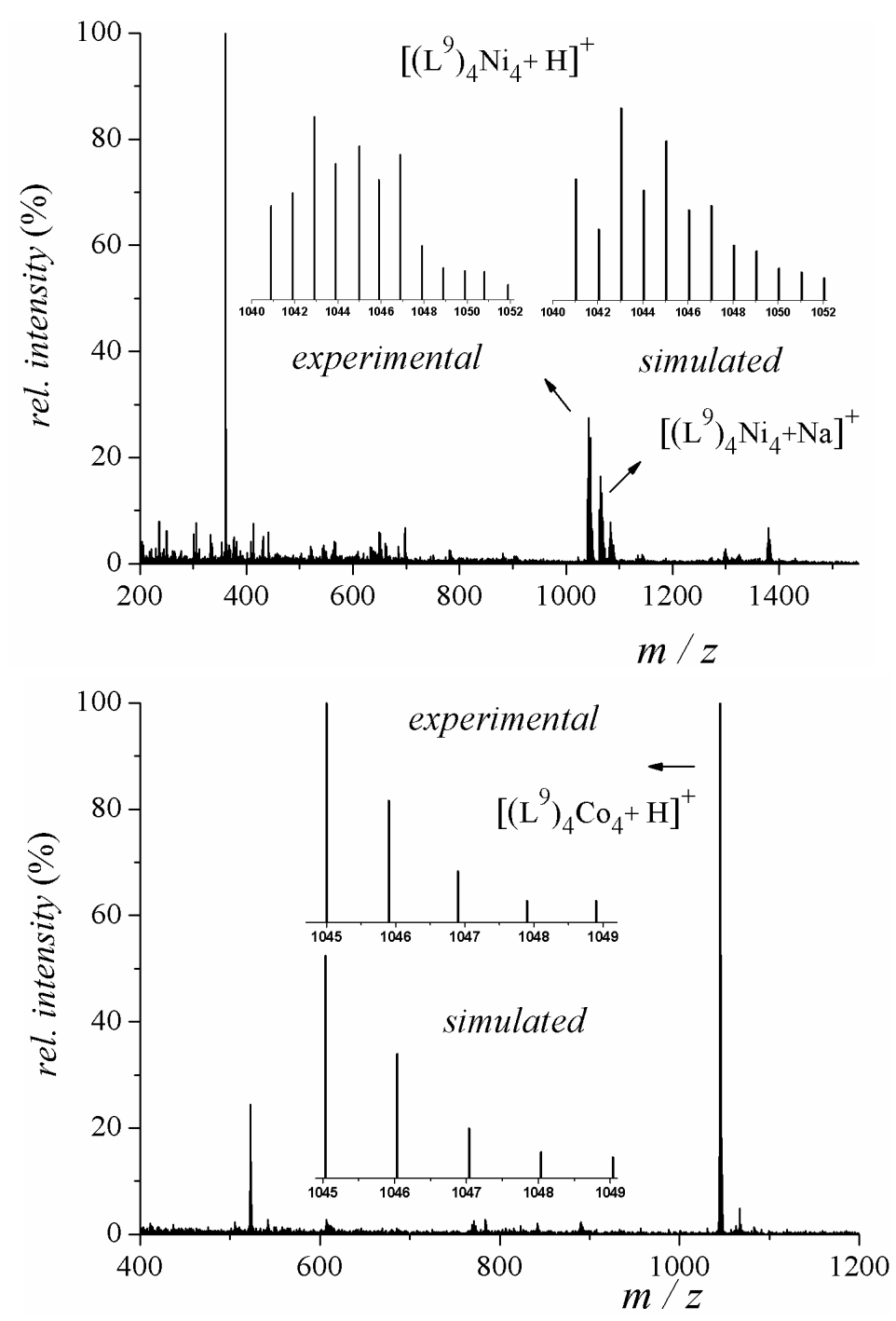

Figure 5.3. Positive ion ESI-MS spectra of $\mathbf{1 1}$ (top) and $\mathbf{1 2}$ (bottom) in $\mathrm{CH}_{2} \mathrm{Cl}_{2}-\mathrm{CH}_{3} \mathrm{OH}$ mixture. The insets show the experimental and expected isotropic distribution pattern for $\left[\left(\mathrm{L}^{9}\right)_{4} \mathrm{Ni}_{4}+\mathrm{H}\right]^{+}$and $\left[\left(\mathrm{L}^{9}\right)_{4} \mathrm{Co}_{4}+\mathrm{H}\right]^{+}$respectively.

5.3.2. UV-visible spectroscopy: The UV-vis spectra of complexes 11,12 , and 13 in $\mathrm{CH}_{2} \mathrm{Cl}_{2}$ (Figure 5.4) showed intense $\pi \rightarrow \pi^{*}$ bands at $335 \mathrm{~nm}, 325$, and $309 \mathrm{~nm}$ respectively, and in addition complex 11 possesses d-d band at $532 \mathrm{~nm}$, and complex 12 displays two weak d-d bands at 561 and $481 \mathrm{~nm}$. Complex 13 exhibits two distinct d-d bands at 517 and $790 \mathrm{~nm}$. 

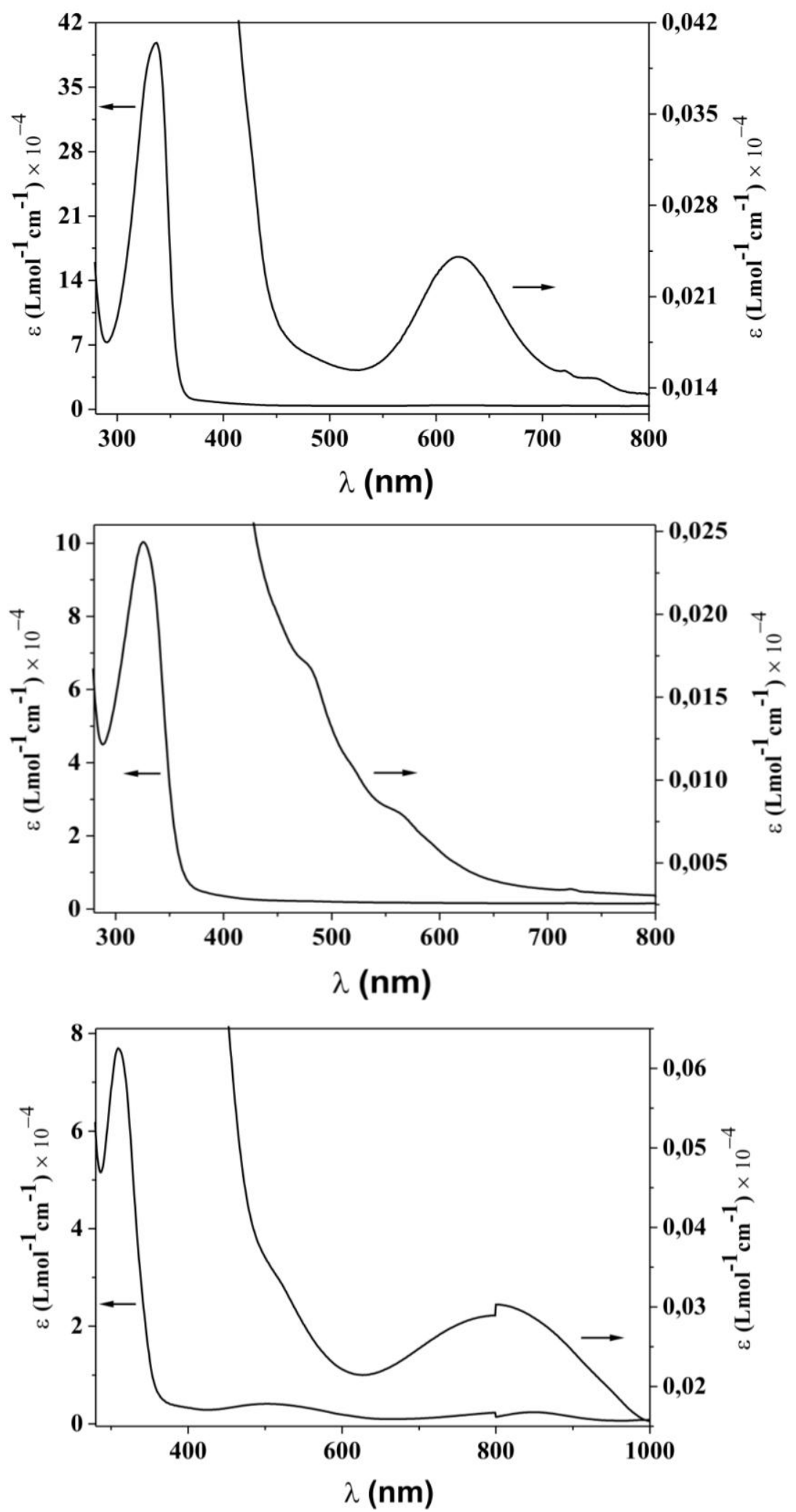

Figure 5.4. UV-visibale spectra of compound $\mathbf{1 1}$ (top), $\mathbf{1 2}$ (middle), and $\mathbf{1 3}$ (bottom) in $\mathrm{CH}_{2} \mathrm{Cl}_{2}$ at room temperature. 
5.3.3. Electrochemistry: Cyclic voltammograms (CVs) of complex 12, and 13 have been recorded in $\mathrm{CH}_{2} \mathrm{Cl}_{2}$, containing $0.10 \mathrm{M}\left[\mathrm{N}(n-\mathrm{Bu})_{4}\right] \mathrm{PF}_{6}$ as a supporting electrolyte (Figure 5.5). Small amounts of decamethyl ferrocene were added after the completion of each set of experiments as an internal standard, and all potentials are referenced versus the $\mathrm{Fc}^{+} / \mathrm{Fc}$ couple.
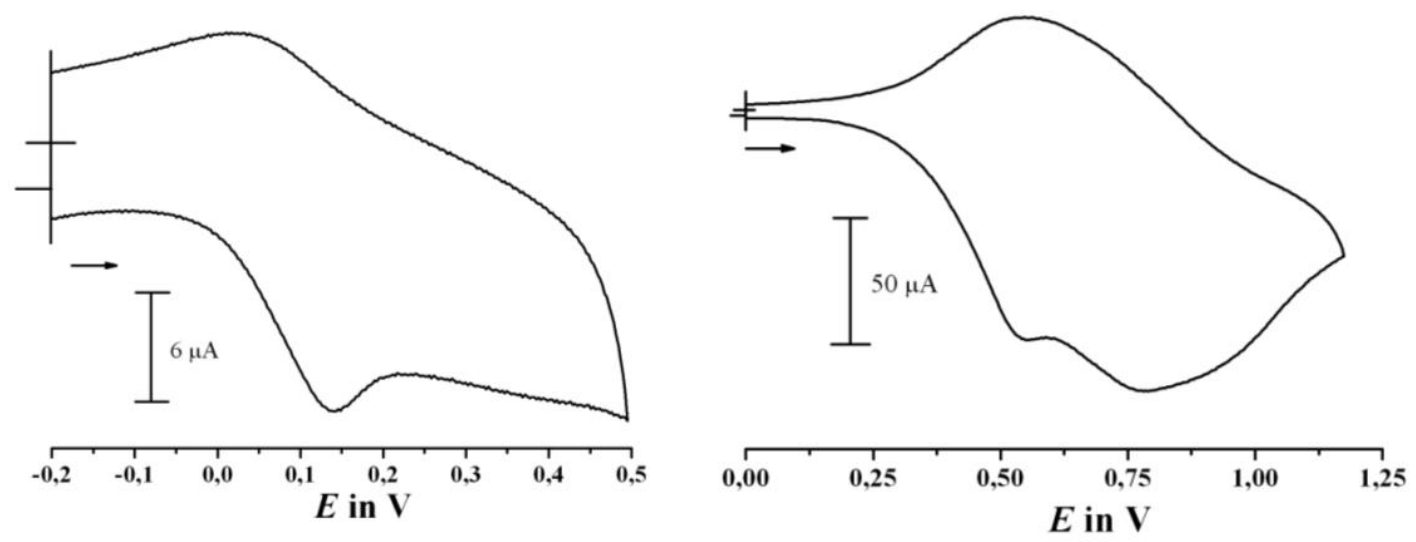

Figure 5.5. Cyclic Voltammograms of complex 12 (in left), and 13 (in right) in $\mathrm{CH}_{2} \mathrm{Cl}_{2}(0.1 \mathrm{M})\left[\left(n-\mathrm{Bu}_{4} \mathrm{~N}\right]\left(\mathrm{PF}_{6}\right)\right.$ at $20{ }^{\circ} \mathrm{C}$ recorded at a glassy carbon working electrode versus a $\mathrm{Ag} / \mathrm{AgNO}_{3}$ reference electrode.

The CVs of complex 12 show an irreversible oxidation wave at $E_{\mathrm{p} \text {, ox }}^{1}=0.14 \mathrm{~V}$, probably for two electron oxidation, and complex 13 displays two irreversible oxidation wave at $E_{\mathrm{p} \text {, ox }}^{1}=$ $0.546 \mathrm{~V}$, and $E_{\mathrm{p} \text {, ox }}^{2}=0.778 \mathrm{~V}$ on the anodic side plausibly owing overall three eletron oxidation occurs in the oxidation cycle. In both cases perhaps metal based oxidation occurs in the oxidation cycle, and $\mathrm{Co}^{2+}$ undergoes to $\mathrm{Co}^{3+}$, and $\mathrm{Fe}^{2+}$ converts to $\mathrm{Fe}^{3+}$. According to the CVs data, redox stability of the ferrous cube is relatievely better in comparision to cobalt cube.

\subsection{Spectroscopy in the solid states}

In order to gain further insight into the electronic structure of the cubane cores, all the compounds were characterized by temperature dependent magnetic susceptibility measurements, and magnetisation measurement. In addition zero-field Mössbauer spectroscopy was performed for compound $\mathbf{1 3}$.

5.4.1. Magnetic properties: Magnetic susceptibility data were collected for complexes 11-13 in the temperature range from 295 to $2.0 \mathrm{~K}$ in order to characterize the exchange coupling within the cubane core. No significant field dependence was observed when data were measured at applied fields of 0.2 and $0.5 \mathrm{~T}$. The temperature dependence of the molar magnetic susceptibility $\chi_{\mathrm{M}}$ and of the product $\chi_{\mathrm{M}} T$ is shown in Figure 5.6. The observed $\chi_{\mathrm{M}} T$ 
value at $295 \mathrm{~K}$ is $5.34 \mathrm{~cm}^{3} \mathrm{Kmol}^{-1}$ (corresponding to an effective moment $\mu_{\mathrm{eff}}=6.54 \mu_{\mathrm{B}}$ ) for 11, $11.06 \mathrm{~cm}^{3} \mathrm{Kmol}^{-1}$ (corresponding to an effective moment $\mu_{\mathrm{eff}}=9.41 \mu_{\mathrm{B}}$ ) for $\mathbf{1 2}, 17.14$ $\mathrm{cm}^{3} \mathrm{Kmol}^{-1}$ (corresponding to an effective moment $\mu_{\mathrm{eff}}=11.71 \mu_{\mathrm{B}}$ ) for $\mathbf{1 3}$ and these are close to the theoretical expected for four uncoupled $\mathrm{M}^{\mathrm{II}}$ ions $\left(\mathrm{hs}-\mathrm{Ni}^{\mathrm{II}} 4.92 \mathrm{~cm}^{3} \mathrm{Kmol}^{-1}\right.$ or $\mu_{\text {eff }}=6.25$ $\mu_{\mathrm{B}}$ for $g=2.22$; hs-Co ${ }^{\mathrm{II}} 11.70 \mathrm{~cm}^{3} \mathrm{Kmol}^{-1}$ or $\mu_{\mathrm{eff}}=9.68 \mu_{\mathrm{B}}$ for $g=2.50$; hs-Fe $\mathrm{Fe}^{\mathrm{II}} 14.37$ $\mathrm{cm}^{3} \mathrm{Kmol}^{-1}$ or $\mu_{\mathrm{eff}}=10.72 \mu_{\mathrm{B}}$ for $g=2.19$ ). Upon lowering the temperature, in the case of $\mathbf{1 2}$ $\chi_{\mathrm{M}}$ goes through a broad maximum at $15 \mathrm{~K}$ while $\chi_{\mathrm{M}} T$ drops and gradually tends to zero, which is indicative of significant antiferromagnetic coupling between the four cobalt(II) ions and an $S=0$ ground state. However in case of 11 and 13, $\chi_{M} T$ value increases gradually with decreasing temperature to reach $7.48 \mathrm{~cm}^{3} \mathrm{Kmol}^{-1}$ (corresponding to an effective moment $\mu_{\text {eff }}=$ $7.73 \mu_{B}$ ) at $8 \mathrm{~K}$ for $\mathbf{1 1}$, and $38.32 \mathrm{~cm}^{3} \mathrm{Kmol}^{-1}$ (corresponding to an effective moment $\mu_{\mathrm{eff}}=$ $\left.17.51 \mu_{B}\right)$ at $10 \mathrm{~K}$ for 13 . This behaviour is consistent with overall ferromagnetic intramolecular interactions between the four metal(II) ions and an $S_{\mathrm{T}}=4$ for complex 11, and $S_{\mathrm{T}}=8$ for complex 13. The decreases of $\chi_{\mathrm{M}} T$ observed at low tempertaure (below $8 \mathrm{~K}$ for complex 11 and $10 \mathrm{~K}$ for complex 13) may be attributed to the effects of ZFS and/or antiferromagnetic intermolecular interactions.

Experimental data were simulated using a fitting procedure to the appropriate HeisenbergDirac-van-Vleck (HDvV) spin Hamiltonian for isotropic exchange coupling and Zeeman

$$
\begin{aligned}
& \hat{H}=-2 J_{1}\left(\hat{S}_{1} \hat{S}_{2}+\hat{S}_{1} \hat{S}_{3}+\hat{S}_{2} \hat{S}_{4}+\hat{S}_{3} \hat{S}_{4}\right)-2 J_{2}\left(\hat{S}_{2} \hat{S}_{3}+\hat{S}_{1} \hat{S}_{4}\right)+ \\
& +g \mu_{B} B \sum_{i=1}^{4} \hat{S}_{z i}+D \sum\left(\hat{S}_{i z}^{2}-\hat{S}_{i}\left(\hat{S}_{i}+1\right) / 3\right)
\end{aligned}
$$

splitting (eq. 5.1) for complexes 11-13, where $J_{2}$ characterises exchange across the two faces of the $\mathrm{M}_{4}$ cubane that are not bridged by hydrogen bonds, while $J_{1}$ characterizes the remaining four pairwise interactions (Scheme 5.3). Table 5.2 summarizes the magnetic parameters obtained from best fit analyses.

\section{Scheme 5.3.}

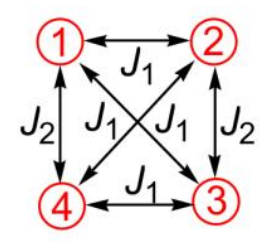



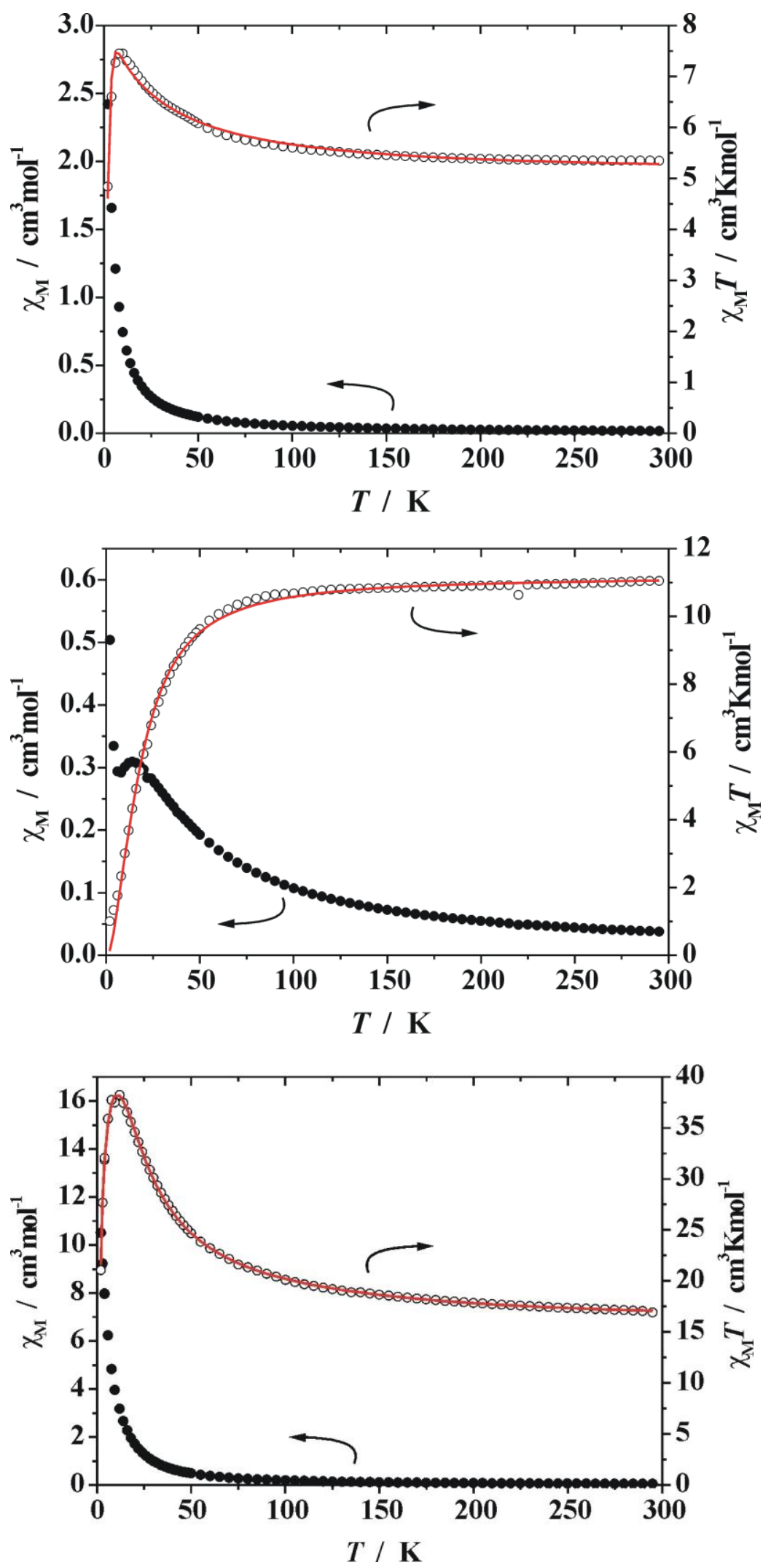

Figure 5.6. Plots of $\chi_{\mathrm{M}}$ (solid circles) and $\chi_{\mathrm{M}} T$ (open circles) versus temperature for $\mathbf{1 1}$ (top), $\mathbf{1 2}$ (middle), and 13 (bottom) at $5000 \mathrm{G}$. The solid red lines represent the simulated curves. 
The $g$ values 2.2 for complex 11, 2.5 for complex 12, and 2.19 for complex $\mathbf{1 3}$ are consistent with those typically observed for other alkoxo bridged cube structures of $\mathrm{Ni}^{\mathrm{II}}, \mathrm{Co}^{\mathrm{II}}$, and $\mathrm{Fe}^{\mathrm{II}}$ respectively. The average values for the three complexes were as follows: $J_{1}=+6.86 \mathrm{~cm}^{-1}, J_{2}$ $=-4.86 \mathrm{~cm}^{-1}, g=2.22$ for complex 11, $J_{1}=+1.57 \mathrm{~cm}^{-1}, J_{2}=-3.94 \mathrm{~cm}^{-1}, g=2.5$ for complex 12, and $J_{1}=+7.04 \mathrm{~cm}^{-1}, J_{2}=-4.90 \mathrm{~cm}^{-1}, g=2.19$ for complex 13.

Table 5.2: Best Fit Parameters of Magnetic Data Analysis for Complexes 11-13

\begin{tabular}{|l|l|l|l|l|l|l|l|}
\hline complex & $g$ & $J_{1}\left[\mathrm{~cm}^{-1}\right]$ & $J_{2}\left[\mathrm{~cm}^{-1}\right]$ & $|D|\left[\mathrm{cm}^{-1}\right]$ & $\rho[\%]$ & $T I P\left[10^{-6} \mathrm{~cm}^{3} \mathrm{~mol}^{-1}\right]$ \\
\hline $\mathbf{1 1}$ & 2.22 & +6.86 & -4.86 & 10 & 0.1 & 227.3 \\
\hline $\mathbf{1 2}$ & 2.50 & +1.57 & -3.94 & 0 & 5 & 400.0 \\
\hline $\mathbf{1 3}$ & 2.19 & +7.04 & -4.90 & 7.5 & 1 & 1907.9 \\
\hline
\end{tabular}

The studies of a number of $\mathrm{M}^{\mathrm{II}}{ }_{4}$ complexes with distorted cubane structures and bridging $\mathrm{O}$ atoms have revealed that the observed exchange interaction between metal centres make a transition from ferromagnetic to aniferromagnetic as the $\mathrm{M}-\mathrm{O}-\mathrm{M}$ bridging angles increase above $98^{\circ}$, and $\mathrm{M}-\mathrm{O}-\mathrm{O}-\mathrm{M}$ dihedral angles increase above $170 .{ }^{63}$ Thus for the present complexes, the four-hydrogen bonded faces of the cubane (characterised by $J_{1}$ ) display angles typical for ferromagnetic interactions, while the remaining two faces (characterised by $J_{2}$ ) possess angles consistent with antiferromagnetic exchange interactions, which gives the different coupling constants (see Table 5.2). In case of $\mathrm{Ni}^{\mathrm{II}}$ and $\mathrm{Fe}_{4}{ }_{4}$ cubes the coupling constant $J_{1}$ is greater than $J_{2}\left(J_{1}>J_{2}\right)$, and that gives overall ferromagnetic coupling and high spin ground states $S_{\mathrm{T}}=4$, and $S_{\mathrm{T}}=8$ respectively, but in case of the $\mathrm{Co}^{\mathrm{II}}{ }_{4}$ cube intramolecular antiferromagnetic coupling $J_{2}$ is greater than the ferromagnetic coupling $J_{1}$ (i.e, $J_{2}>J_{1}$ ), which finally gives an $S_{\mathrm{T}}=0$ ground state. This could be due to little distortion of the bond angles of Co-O-Co across the two non hydrogen bonded faces compared to the other complexes (see Table 5.1). The field dependence magnetization (0-5 T) of compound 11-13 measured at $2 \mathrm{~K}$ is shown in Figure 5.7 in the form of $M / N \beta$ vs. $H$. In case of the $\mathrm{Ni}^{\mathrm{II}}$ compound $\mathbf{1 1}$ and $\mathrm{Fe}^{\mathrm{II}}$ compound 13, no any true saturation occur upto $5 \mathrm{~T}$, it rises, and may be saturate at higher magnetic field. The magnetization reaches a value of $\sim 7.3 N \beta$ (for 11) and $\sim 16.5 N \beta$ (for 13) at $5 \mathrm{~T}$, these are close to the expected $S=4$ value of $8 N \beta$ for the $\mathrm{Ni}_{4}$ system, and $S=8$ value of $16 N \beta$ for the $\mathrm{Fe}_{4}$ system indicating the presence of ferromagnetic interaction between the neighbouring metal(II) ions. ${ }^{64}$ 

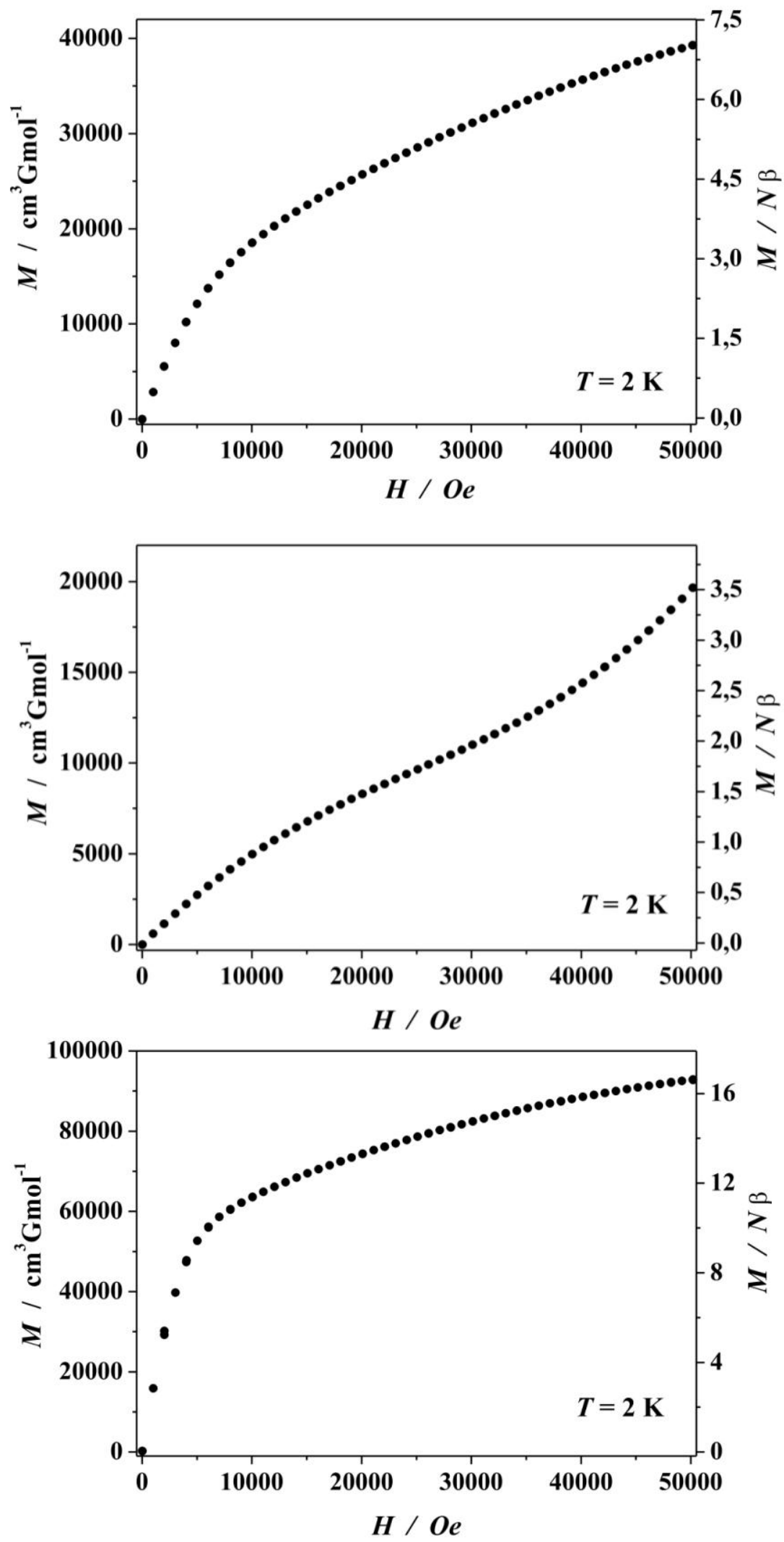

Figure 5.7. Plot of magnetisation vs $H$ at $2 \mathrm{~K}$ for complex 11 (top), 12 (middle), and $\mathbf{1 3}$ (bottom). 
The magnetisation curve of the cobalt complex shows an unusual behavior: it increases at first almost linearly with the magnetic field, and then rises; it may be saturate at higher magnetic fields, which is consistent with metamagnetic-like behavior. Such kind of property is nicely fitted with recently reported square gird complex of cobalt(II) with bis(bipyridyl)-pyrimidinebased ligand system. ${ }^{65}$

5.4.3. Mössbauer spectroscopy: A Mössbauer spectrum of compound 13 was recorded at 80 $\mathrm{K}$ and the spectrum is shown in Figure 5.8. Spectral fits to the data are obtained by using Lorentzian line doublets with isomer shifts $(\delta)$ and quadrupole splitting $\left(\Delta E_{\mathrm{Q}}\right)$ summarized in Table 5.3. The isomer shifts $(\delta)$ of 1.23 , and quadrupole splitting $\left(\Delta E_{\mathrm{Q}}\right)$ of 2.73 clearly idicates that all the iron atoma are equivalent and high-spin Fe(II). ${ }^{66}$ The high $\Delta E_{\mathrm{Q}}$ signifies an important axial distortion which can correspond to the long Fe-O bond.

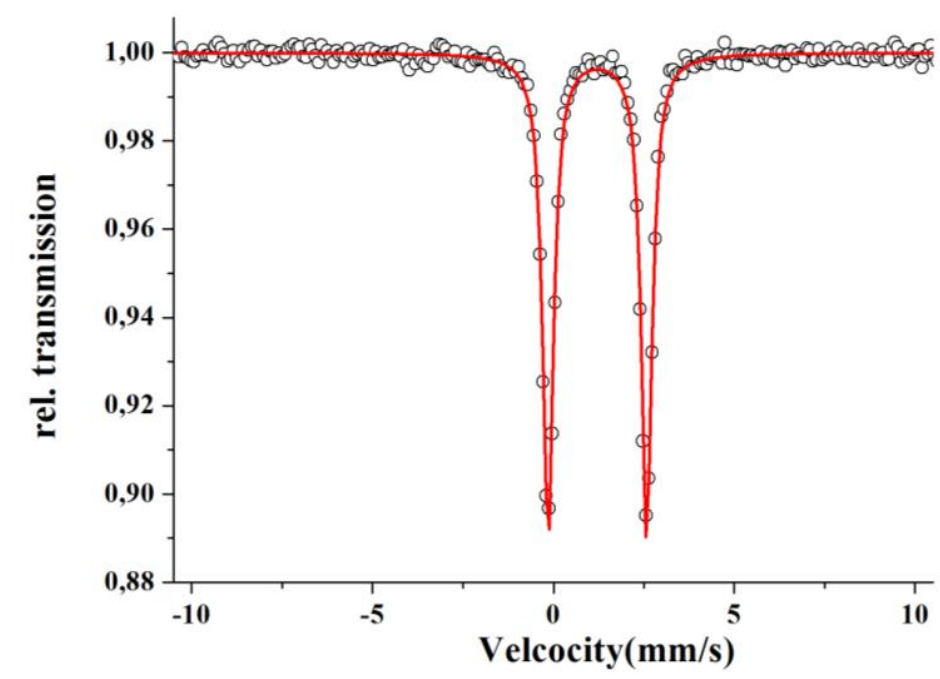

Figure 5.8. Mössbauer spectra of 13 recorded in zero field at $80 \mathrm{~K}$. Red solid curve is fitted to the experimental values (circles).

Table.5.3. Mössbauer spectral data for 13.

\begin{tabular}{|l|l|l|l|l|}
\hline$T(\mathrm{~K})$ & $\delta\left(\mathrm{nm} \mathrm{s}^{-1}\right)$ & $\Delta E_{\mathrm{Q}}\left(\mathrm{nm} \mathrm{s}^{-1}\right)$ & $\Gamma_{1 / 2}\left[\mathrm{~mm} \mathrm{~s}^{-1}\right]$ & Area ratio $(\%)$ \\
\hline 80 & 1.21 & 2.73 & 0.36 & 100 \\
\hline
\end{tabular}




\subsection{Conclusions}

In this chapter we have described series of new phenol-pyrazole based tridentate diol-ligands, in which to confirm the integrity of pyrazole core one of the ligand $\mathbf{H}_{2} \mathbf{L}^{9}$ was confirmed by Xray diffraction analysis. It forms a propeller-like hydrogen-bonded tetramer via the aliphatic OH groups. In presence of weak donor ligand methanol, $\mathbf{H}_{2} \mathbf{L}^{\mathbf{9}}$ shows a high tendency toward the selective formation of stable tetranuclear cube structures with metal ions such as $\mathrm{Ni}^{\mathrm{II}}, \mathrm{Co}^{\mathrm{II}}$, and $\mathrm{Fe}^{\mathrm{II}}$. The molecular structures of the $\left[\mathrm{Ni}_{4}(\mathrm{MeOH})_{4}\left(\mathrm{~L}^{9}\right)_{4}\right](\mathbf{1 1}),\left[\mathrm{Co}_{4}(\mathrm{MeOH})_{3}\left(\mathrm{H}_{2} \mathrm{O}\right)\left(\mathrm{L}^{9}\right)_{4}\right]$ (12), and $\left[\mathrm{Fe}_{4}(\mathrm{MeOH})_{4}\left(\mathrm{~L}^{9}\right)_{4}\right](\mathbf{1 3})$ posses distorted octahedral geometry around the metal centers. No dissociation or scrambling of the cube cores is observed in solution. Magnetic susceptibility and magnetisation measurements show a combination of ferromagnetic and antiferromagnetic pair wise exchange interactions are operative between the orthogonal arrangements of the $\mathrm{M}^{\mathrm{II}}$ centre in the metal cubes, leading to high spin ground state $S_{\mathrm{T}}=4$ for complex 11, and $S_{\mathrm{T}}=8$ for complex 13, and on the other side metamagnet like behavior are operative with $\mathrm{Co}^{\mathrm{II}}$ as the spin carrier in complex $\mathbf{1 2}$. 


\section{Chapter 6}

\section{A New Family of Heterometal Single Molecule Magnets: Observation of Quantum Tunnelling Steps in the Hysteresis Loops of Linear $\left\{\mathrm{Mn}_{2} \mathrm{Ni}_{3}\right\}$ Clusters}

\subsection{Introduction}

Since the discovery of the first SMM of a dodecanuclear manganese complex ${ }^{5}$ in 1993, various derivatives of this so-called $\mathrm{Mn}_{12}$ archetype have been investigated, ${ }^{22}$ and much effort has been devoted to the synthesis and characterisation of new polynuclear clusters that display slow magnetic relaxation below a certain blocking temperature $\left(T_{\mathrm{B}}\right)$. There are now many examples of homometal SMMs, mostly containing $\mathrm{Mn}^{\mathrm{III}}$, but also other metal ions such as $\mathrm{V}$, $\mathrm{Fe}, \mathrm{Co}$, or Ni. Still, the $\mathrm{Mn}_{12}$ family $^{22}$ represented the SMMs with the highest $T_{\mathrm{B}}(\sim 3.5 \mathrm{~K})$ and anisotropy barriers $U_{\text {eff }}$ (up to $74 \mathrm{~K}$ ) until 2007, when a $\mathrm{Mn}_{6}$ complex with enhanced blocking temperature $(\sim 4.5 \mathrm{~K})$ and an effective energy barrier to magnetization reversal of $86.4 \mathrm{~K}$ was found, the record holder to date. ${ }^{67,68}$

Of particular value are species that exist in structurally related families, where the subtle effects of structural or electronic variation on the SMM properties can be investigated. In addition, although by definition the individual molecules should be well isolated for the manifestation of SMM behaviour, the molecular properties that are required for a sufficiently large energy barrier to magnetization reversal do not preclude the presence of intermolecular interactions. Thus, several complexes that feature intermolecular contacts in the form of either pairwise $^{69}$ or extended interactions have been found to display slow relaxation of the magnetization associated with an energy barrier of molecular origin, but with the quantum tunnelling of magnetisation (QTM) shifted away from zero field. ${ }^{70}$ These are so-called "exchange-biased SMMs", and they represent useful subjects for the study of the effects of the intermolecular interactions on the behaviour arising from the energy barrier to magnetization reversal. Moreover, it has been suggested that such intermolecular interactions may provide a means of fine-tuning the quantum tunnelling of magnetization in SMMs. ${ }^{71,72}$ Modulation of the intermolecular interactions clearly requires proper adjustment of ligand functionalities in the oligometallic clusters.

The combination of different metal ions in a single cluster for achieving large magnetic anisotropy and high spin ground states significantly extends the structural landscape for SMMs, but has been explored relatively little until now. ${ }^{73,25 c}$ In present work, attempts were made to explore new exchange biased heterometal SMMs using self-assembly of mixed 
$\mathrm{Mn}^{\mathrm{III}} / \mathrm{Ni}^{\mathrm{II}}$ metal ions with tridentate diol ligand 2-[3-(2-hydroxyphenyl)-1H-pyrazol-1yl]ethanol $\left(\mathbf{H}_{\mathbf{2}} \mathbf{L}^{\mathbf{9}}\right)$.

\subsection{Synthesis and structural characterisation of metal complexes}

Reaction of $\mathbf{H}_{2} \mathbf{L}^{9}$ with $\mathrm{MnX}_{2} \cdot \mathrm{XH}_{2} \mathrm{O}(\mathrm{X}=\mathrm{Cl}, \mathrm{Br})$ and $\mathrm{NiCl}_{2} \cdot 6 \mathrm{H}_{2} \mathrm{O}$ in the presence of $\mathrm{NEt}_{3}$ in acetonitrile at room temperature gave complexes $\left[\mathrm{Mn}^{\mathrm{III}}{ }_{2} \mathrm{Ni}_{3} \mathrm{X}_{2}\left(\mathrm{~L}^{9}\right)_{4}\left(\mathrm{HL}^{9}\right)_{2}\left(\mathrm{H}_{2} \mathrm{O}\right)_{2}\right](\mathrm{X}=\mathrm{Cl}$ : 14; $\mathrm{X}=\mathrm{Br}$ : 15). Both compounds could be crystallized from dichloromethane solutions as $14 \cdot 8 \mathrm{CH}_{2} \mathrm{Cl}_{2}$ or $15 \cdot 8 \mathrm{CH}_{2} \mathrm{Cl}_{2}$, respectively, and 14 could also be obtained in crystalline form from 1,2-dichloroethane solution as $\mathbf{1 4} \cdot 6 \mathrm{C}_{2} \mathrm{H}_{4} \mathrm{Cl}_{2}$. In all cases the complexes crystallize in the triclinic space group $P-1$ as quasi-linear centrosymmetric Mn-Ni-Ni-Ni-Mn molecules, with the central nickel atom situated on a centre of inversion. Overall molecular topologies determined by X-ray crystallography are very similar, and the molecular structure of $\mathbf{1 4}$ from both solvates and $\mathbf{1 5}$ are shown in Figure 6.1. Relevant atom distances and bond angles and collected in Table 6.1. Both complexes contain a $\left[\mathrm{Mn}_{2} \mathrm{Ni}_{3}(\mu-\mathrm{O})_{8}\right]$ core where the coordination number of all metal atoms is six and the coordination environment is more or less distorted octahedral. Complexes 14 and 15 differ only by the terminal ligands bound to $\mathrm{Mn}$, which are either $\mathrm{Cl}$ in $\mathbf{1 4}$ or $\mathrm{Br}$ in $\mathbf{1 5}$. Oxidation states of the metal ions, $\mathrm{Mn}^{\mathrm{III}}$ and $\mathrm{Ni}^{\mathrm{II}}$, are confirmed by bond valence sum calculations ${ }^{74}$ and by the relatively short bonds for the $\mathrm{Mn}^{\mathrm{III}}$ ions (see Table 6.1). The string of metal ions can be best visualized as five edge-sharing octahedra (Figure 6.2 , bottom). The three $\mathrm{Ni}^{\mathrm{II}}$ ions are doubly bridged by phenoxy-O atoms, while both peripheral $\mathrm{Mn}^{\mathrm{III}}$ ions are linked to the $\mathrm{Ni}_{3}{ }_{3}$ core via double alkoxy-O bridging. $\mathrm{Mn}^{\mathrm{III}}$ ions are found in $\left\{\mathrm{NO}_{4} \mathrm{X}\right\}$ environment with equatorial position occupied by the $\{\mathrm{ONO}\}$ donor site of a tridentate ligand $\left[\mathbf{L}^{9}\right]^{2-}$ and an alkoxo-O of another ligand, and with the terminal halide $(\mathrm{Cl}$ or $\mathrm{Br}$ ) and a water molecule in the axial positions. As expected, the $\mathrm{Mn}^{\mathrm{III}}$ ions exhibit a strong Jahn-Teller elongation along the $\mathrm{Cl} / \mathrm{Br}-\mathrm{Mn}-\mathrm{OH}_{2}$ axis (Figure 6.2, top and middle) with $\mathrm{Mn}-$ $\mathrm{O} / \mathrm{Cl}$ distances of $2.34 / 2.61 \AA$ in $\mathbf{1 4} \cdot 8 \mathrm{CH}_{2} \mathrm{Cl}_{2}, 2.36 / 2.62 \AA$ in $\mathbf{1 4} \cdot 6 \mathrm{C}_{2} \mathrm{H}_{4} \mathrm{Cl}_{2}$, and $\mathrm{Mn}-\mathrm{O} / \mathrm{Br}$ distances of $2.34 / 2.78 \AA$ in $\mathbf{1 5} \cdot 8 \mathrm{CH}_{2} \mathrm{Cl}_{2}$. In case of nickel the octahedral environment is more regular with the central $\mathrm{Ni}^{\mathrm{II}}$ featuring a $\left\{\mathrm{NO}_{5}\right\}$ and the other $\mathrm{Ni}^{\mathrm{II}}$ a $\left\{\mathrm{N}_{2} \mathrm{O}_{4}\right\}$ coordination sphere. Interestingly, two of the six pyrazole-based ligands in $\mathbf{1 4}$ and $\mathbf{1 5}$ are only deprotonated once. 

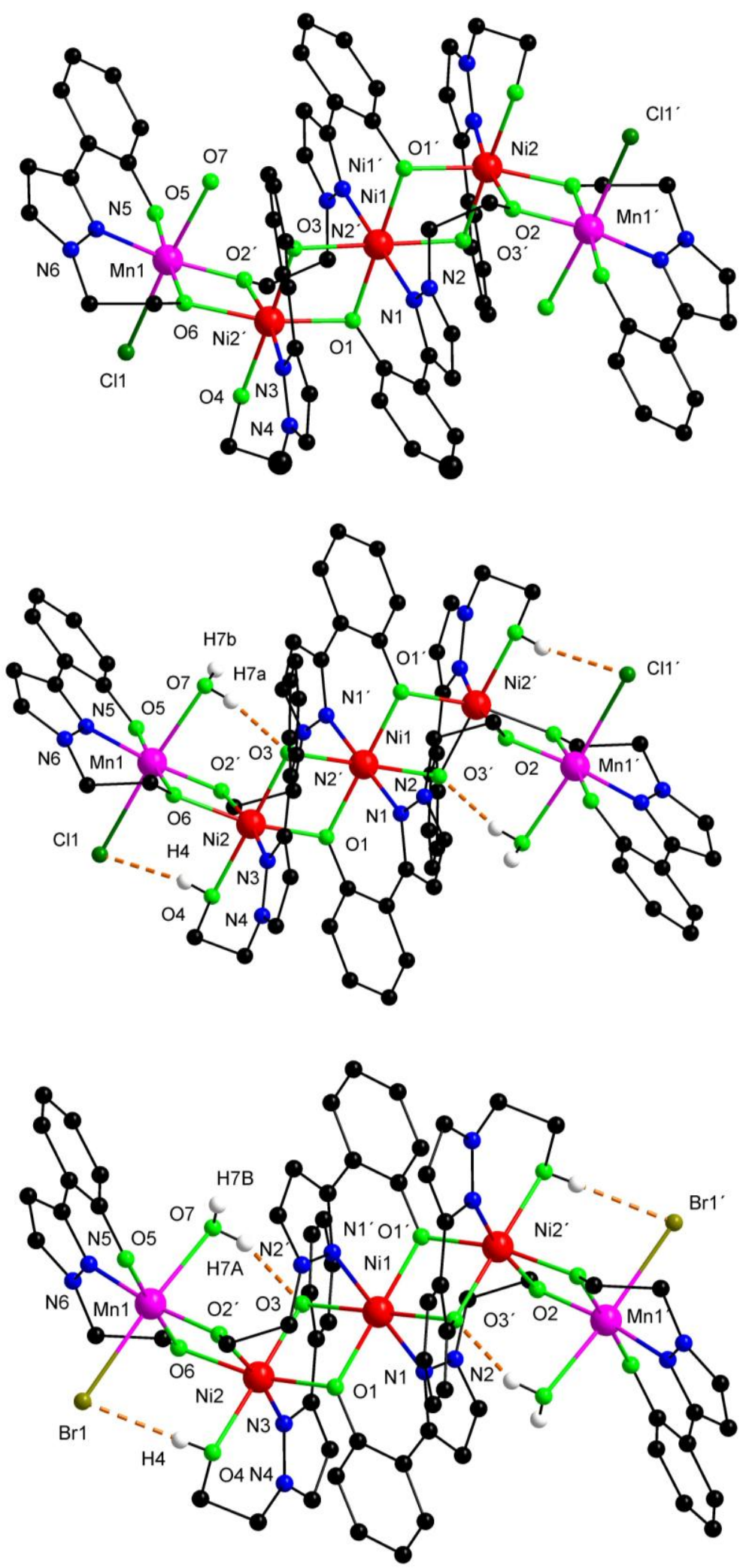

Figure 6.1. Molecular structure of compound 14 in $14 \cdot 8 \mathrm{CH}_{2} \mathrm{Cl}_{2}$ (top), 14 in $14 \cdot 6 \mathrm{C}_{2} \mathrm{H}_{4} \mathrm{Cl}_{2}$ (middle), 15 in 15. $8 \mathrm{CH}_{2} \mathrm{Cl}_{2}$ (bottom). Most hydrogen atoms and solvent molecules are omitted for clarity. Symtmetry transformation used to generate equivalent atoms: (') 1-x, 1-y, 1-z. 

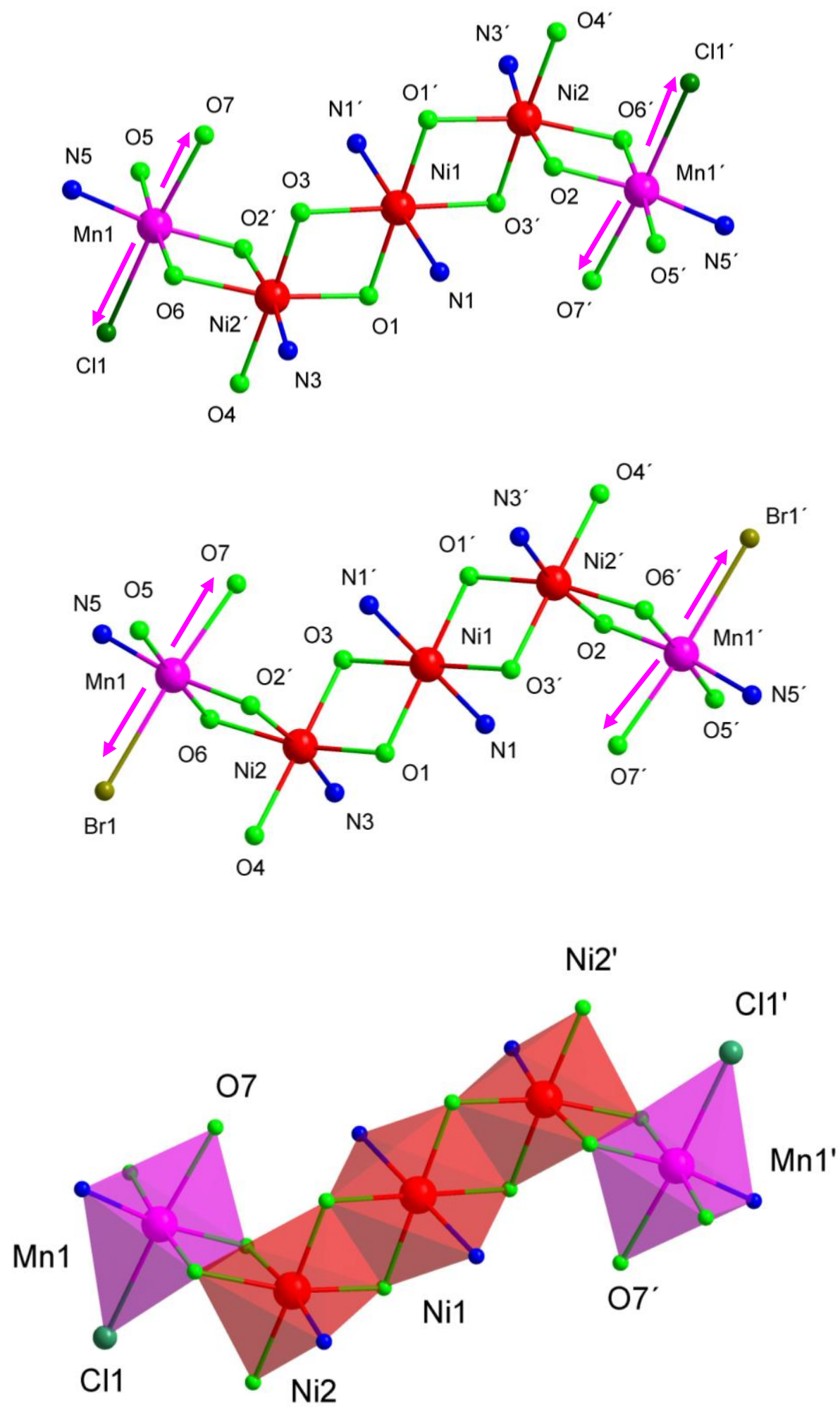

Figure 6.2. Coordination environment of the metal atoms of 14 in $14 \cdot 6 \mathrm{C}_{2} \mathrm{H}_{4} \mathrm{Cl}_{2}$ (top), 15 in $15 \cdot 8 \mathrm{CH}_{2} \mathrm{Cl}_{2}$ (middle). The arrows emphasize the Jahn-Teller axes. Symmetry transformation used to generate equivalent atoms: (') $1-x, 1-y, 1-z$. Bottom: Emphasis of the five edge-sharing octahedral of the $\mathrm{Mn}^{\mathrm{III}}-\mathrm{Ni}^{\mathrm{II}}-\mathrm{Ni}^{\mathrm{II}}-\mathrm{Ni}^{\mathrm{II}}-\mathrm{Mn}^{\mathrm{III}}$ core in $\mathbf{1 4 .}$ 
Table 6.1. Selected distances $[\AA]$ and angles $\left[{ }^{\circ}\right]$ for $\mathbf{1 4} \cdot 8 \mathrm{CH}_{2} \mathrm{Cl}_{2}, \mathbf{1 4} \cdot 6 \mathrm{C}_{2} \mathrm{H}_{4} \mathrm{Cl}_{2}$ and $15 \cdot 8 \mathrm{CH}_{2} \mathrm{Cl}_{2}^{\mathrm{a}}$

\begin{tabular}{|c|c|c|c|}
\hline compound & $\mathbf{1 4} \cdot 8 \mathrm{CH}_{2} \mathrm{Cl}_{2}$ & $\mathbf{1 4} \cdot 6 \mathrm{C}_{2} \mathrm{H}_{4} \mathrm{Cl}_{2}$ & $\mathbf{1 5} \cdot 8 \mathrm{CH}_{2} \mathrm{Cl}_{2}$ \\
\hline Ni1-N1 & $2.173(7)$ & $2.176(2)$ & $2.186(4)$ \\
\hline $\mathrm{Ni1}-\mathrm{O} 1$ & $2.030(6)$ & $2.015(2)$ & $2.026(3)$ \\
\hline $\mathrm{Ni1}-\mathrm{O} 3$ & $2.120(6)$ & $2.123(2)$ & $2.116(3)$ \\
\hline Ni2-N3 & $2.022(7)$ & $2.016(2)$ & $2.030(4)$ \\
\hline $\mathrm{Ni2}-\mathrm{O} 1$ & $2.014(6)$ & $2.022(2)$ & $2.012(3)$ \\
\hline $\mathrm{Ni} 2-\mathrm{O} 2$ ' & $2.062(6)$ & $2.060(2)$ & $2.065(3)$ \\
\hline $\mathrm{Ni2}-\mathrm{O} 3$ & $2.061(6)$ & $2.048(2)$ & $2.056(3)$ \\
\hline $\mathrm{Ni} 2-\mathrm{O} 4$ & $2.121(6)$ & $2.099(2)$ & $2.127(3)$ \\
\hline $\mathrm{Ni} 2-\mathrm{O} 6$ & $2.043(6)$ & $2.045(2)$ & $2.047(3)$ \\
\hline Mn1-N5 & $1.977(7)$ & $1.986(2)$ & $1.994(4)$ \\
\hline $\mathrm{Mn} 1-\mathrm{O} 2$ & $1.889(6)$ & $1.893(2)$ & $1.891(3)$ \\
\hline Mn1-O5 & $1.872(6)$ & $1.859(2)$ & $1.866(4)$ \\
\hline Mn1-O6 & $1.888(6)$ & $1.886(2)$ & $1.886(3)$ \\
\hline Mn1-O7 & $2.344(6)$ & $2.357(2)$ & $2.337(4)$ \\
\hline Mn1-X1 & $2.609(3)$ & $2.6196(9)$ & $2.7775(9)$ \\
\hline $\mathrm{Ni} 1 \cdots \mathrm{Ni} 2$ & $3.1089(11)$ & $3.0904(4)$ & $3.1106(6)$ \\
\hline $\mathrm{Ni} 2 \cdots \mathrm{Mn} 1$ & $3.0447(17)$ & $3.0339(5)$ & $3.0457(10)$ \\
\hline $\mathrm{O} 4 \cdots \mathrm{X} 1$ & $3.17(1)$ & $3.238(4)$ & $3.238(4)$ \\
\hline $\mathrm{O} 7 \cdots \mathrm{O} 3$ & $2.77(1)$ & $2.757(5)$ & $2.757(5)$ \\
\hline N1-Ni1-N1', & & 180 & \\
\hline O1-Ni1-O1', & & 180 & \\
\hline O3-Ni1-O3' & & 180 & \\
\hline $\mathrm{N} 3-\mathrm{Ni} 2-\mathrm{O} 2$ & $171.7(3)$ & $170.8(1)$ & $170.5(1)$ \\
\hline $\mathrm{O} 1-\mathrm{Ni2}-\mathrm{O} 6$ & $164.7(2)$ & $164.8(1)$ & $165.0(1)$ \\
\hline $\mathrm{O} 3-\mathrm{Ni} 2-\mathrm{O} 4$ & $177.9(2)$ & 179.1(1) & 178.9(1) \\
\hline N5-Mn1-O2' & $167.2(3)$ & $171.6(1)$ & $169.4(2)$ \\
\hline O5-Mn1-O6 & $175.9(3)$ & $175.6(1)$ & $177.6(2)$ \\
\hline O7-Mn1-X1 & $175.4(2)$ & $173.7(1)$ & $174.5(1)$ \\
\hline $\mathrm{O} 4-\mathrm{H} 4 \cdots \mathrm{X} 1$ & - & $170(4)$ & $175(5)$ \\
\hline $\mathrm{O} 7-\mathrm{H} 7 \mathrm{~A} \cdots \mathrm{O} 3$ & - & $168(5)$ & $154(7)$ \\
\hline
\end{tabular}

${ }^{\mathrm{a}}$ Symmetry transformation used to generate equivalent atoms: (') $1-x, 1-y, 1-z \cdot{ }^{b} \mathrm{X}=\mathrm{Cl}$ : 14; $\mathrm{X}=\mathrm{Br}$ : 15

The remaining neutral alcohol function of these partially deprotonated $\left[\mathbf{H L}^{\mathbf{9}}\right]^{-}$serves as a terminal ligand to the $\mathrm{Ni}_{3}{ }_{3}$ core and exhibits a strong intramolecular $\mathrm{H}$-bond to the neighbouring Mn-bound halide ion $\left[d(\mathrm{O} 4 \cdots \mathrm{Cl} 1)=3.17(1) \AA\right.$ in $\mathbf{1 4} \cdot 8 \mathrm{CH}_{2} \mathrm{Cl}_{2}$ and 3.238(4) $\AA$ in 14. $6 \mathrm{C}_{2} \mathrm{H}_{4} \mathrm{Cl}_{2} ; d(\mathrm{O} 4 \cdots \mathrm{Br} 1)=3.238(4) \AA$ in $\left.\mathbf{1 5} \cdot 8 \mathrm{CH}_{2} \mathrm{Cl}_{2}\right] ;{ }^{75}$ the $\mathrm{Ni}-\mathrm{O} 4$ bond is elongated compared to all other Ni2-O and Ni2-N bonds (2.10- $2.13 \AA$ versus 2.01- $2.07 \AA$ ). Another important intramolecular $\mathrm{H}$-bond involves the $\mathrm{Mn}^{\mathrm{III}}$-bound water and the proximate phenolato-O3 atom $[d(\mathrm{O} 7 \cdots \mathrm{O} 3) \approx 2.76 \AA]$. These two H-bonding interactions displayed by the axial ligands at $\mathrm{Mn}$ fix the $\mathrm{Cl} / \mathrm{Br}-\mathrm{Mn}-\mathrm{OH}_{2}$ axis relative to the $\mathrm{O} 3-\mathrm{Ni2}-\mathrm{O} 4$ axis, i.e., the $\mathrm{Mn} 1$ and $\mathrm{Ni} 2$ coordination octahedra have their elongated axes aligned parallel. 
Table 6.2. Selected intermolecular distances $[\AA]$ for $\mathbf{1 4} \cdot 8 \mathrm{CH}_{2} \mathrm{Cl}_{2}, \mathbf{1 4} \cdot 6 \mathrm{C}_{2} \mathrm{H}_{4} \mathrm{Cl}_{2}$ and $15 \cdot 8 \mathrm{CH}_{2} \mathrm{Cl}_{2}$

\begin{tabular}{|c|c|c|c|c|c|}
\hline Atom1 & Atom2 & Symm. op. 1 & Symm. op. 2 & Distance & Length-VdW \\
\hline \multicolumn{6}{|c|}{$\mathbf{1 4} \cdot 8 \mathrm{CH}_{2} \mathrm{Cl}_{2}$ : } \\
\hline $\mathrm{Cl1}$ & H61A & $x, y, z$ & $1-x, 1-y, 2-z$ & 2.664 & -0.286 \\
\hline O5 & H62A & $x, y, z$ & $1-x, 1-y, 2-z$ & 2.401 & -0.319 \\
\hline O7 & H64B & $x, y, z$ & $x, 1+y, z$ & 2.385 & -0.335 \\
\hline $\mathrm{Cl} 4$ & H46 & $x, y, z$ & $1-x, 2-y, 2-z$ & 2.935 & -0.015 \\
\hline $\mathrm{Cl} 2$ & H61B & $x, y, z$ & $x, y, z$ & 2.894 & -0.056 \\
\hline $\mathrm{Cl} 3$ & H43 & $x, y, z$ & $-1+x,-1+y, z$ & 2.737 & -0.213 \\
\hline $\mathrm{Cl} 2$ & H61B & $x, y, z$ & $1-x, 1-y, 2-z$ & 2.872 & -0.078 \\
\hline $\mathrm{Cl} 7$ & $\mathrm{H} 51 \mathrm{~B}$ & $x, y, z$ & $-1+x, y, z$ & 2.926 & -0.024 \\
\hline \multicolumn{6}{|c|}{ 14.6 $\mathrm{C}_{2} \mathrm{H}_{4} \mathrm{Cl}_{2}$ : } \\
\hline $\mathrm{Cl1}$ & $\mathrm{H} 41$ & $x, y, z$ & $-x,-y,-z$ & 2.836 & -0.114 \\
\hline $\mathrm{Cl} 1$ & H61A & $x, y, z$ & $x, y,-1+z$ & 2.788 & -0.162 \\
\hline O5 & H62A & $x, y, z$ & $x, y,-1+z$ & 2.593 & -0.127 \\
\hline $\mathrm{Cl1}$ & H63A & $x, y, z$ & $x, y,-1+z$ & 2.938 & -0.012 \\
\hline $\mathrm{Cl} 4 \mathrm{~A}$ & $\mathrm{H} 10 \mathrm{~A}$ & $x, y, z$ & $x, y, z$ & 2.835 & -0.115 \\
\hline $\mathrm{Cl} 6 \mathrm{~A}$ & $\mathrm{H} 23$ & $x, y, z$ & $x, y, z$ & 2.781 & -0.169 \\
\hline $\mathrm{Cl6B}$ & $\mathrm{H} 23$ & $x, y, z$ & $x, y, z$ & 2.840 & -0.110 \\
\hline $\mathrm{Cl} 3$ & $\mathrm{H} 43$ & $x, y, z$ & $-x,-y, 1-z$ & 2.946 & -0.004 \\
\hline $\mathrm{Cl} 4 \mathrm{~A}$ & H50B & $x, y, z$ & $1-x,-y, 1-z$ & 2.935 & -0.015 \\
\hline $\mathrm{Cl} 4 \mathrm{~A}$ & H61B & $x, y, z$ & $1-x, 1-y, 2-z$ & 2.889 & -0.061 \\
\hline Cl4B & H50B & $x, y, z$ & $1-x,-y, 1-z$ & 2.907 & -0.043 \\
\hline Cl7A & $\mathrm{H} 21$ & $x, y, z$ & $2-x,-y, 1-z$ & 2.872 & -0.078 \\
\hline \multicolumn{6}{|c|}{$\mathbf{1 5} \cdot 8 \mathrm{CH}_{2} \mathrm{Cl}_{2}$ : } \\
\hline Br1 & H31A & $x, y, z$ & $2-x, 1-y, 2-z$ & 3.035 & -0.015 \\
\hline $\mathrm{Br} 1$ & H41 & $x, y, z$ & $2-x, 2-y, 2-z$ & 2.998 & -0.052 \\
\hline Br1 & H61B & $x, y, z$ & $1-x, 1-y, 2-z$ & 2.992 & -0.058 \\
\hline $\mathrm{Br} 1$ & H62B & $x, y, z$ & $1-x, 1-y, 2-z$ & 3.022 & -0.028 \\
\hline O5 & H62B & $x, y, z$ & $1-x, 1-y, 2-z$ & 2.456 & -0.264 \\
\hline $\mathrm{O} 7$ & H64A & $x, y, z$ & $x, 1+y, z$ & 2.579 & -0.141 \\
\hline $\mathrm{Cl} 2$ & H61B & $x, y, z$ & $x, y, z$ & 2.947 & -0.003 \\
\hline $\mathrm{Cl} 3$ & $\mathrm{H} 43$ & $x, y, z$ & $-1+x,-1+y, z$ & 2.849 & -0.101 \\
\hline
\end{tabular}

With respect to the magnetic properties it is also important to note that the Jahn-Teller axes of the $\mathrm{Mn}^{\mathrm{III}}$ ions at both ends of the $\mathrm{Mn}^{\mathrm{III}}-\mathrm{Ni}^{\mathrm{II}}-\mathrm{Ni}^{\mathrm{II}}-\mathrm{Ni}^{\mathrm{II}}-\mathrm{Mn}^{\mathrm{III}}$ core are roughly parallel to each other. Since classic hydrogen bonds in $\mathbf{1 4}$ and $\mathbf{1 5}$ are only intramolecular, intermolecular interaction might occur via short $\mathrm{C}-\mathrm{H} \cdots \mathrm{X}(\mathrm{X}=\mathrm{Cl}, \mathrm{Br}, \mathrm{O})$ contacts. In this context short contacts are defined as a distance shorter than the sum of the van der Waals radii of the respective atoms with a $\mathrm{C}-\mathrm{H} \cdots \mathrm{X}$ angle greater than $90^{\circ}$. Such distances were determined using the program Mercury ${ }^{76}$ and are listed in Table 6.2.

Interestingly within the given range no direct molecular contacts of two or more $\left[\mathrm{Mn}_{2}{ }_{2} \mathrm{Ni}_{3}{ }_{3} \mathrm{Cl}_{2}\left(\mathrm{~L}^{9}\right)_{4}\left(\mathrm{HL}^{9}\right)_{2}\left(\mathrm{H}_{2} \mathrm{O}\right)_{2}\right]$ units are observed for $\mathbf{1 4} \cdot 8 \mathrm{CH}_{2} \mathrm{Cl}_{2}$. Instead the $\mathrm{CH}_{2} \mathrm{Cl}_{2}$ 
solvent molecules containing the atoms $\mathrm{Cl} 2 / 3$ and $\mathrm{Cl} 4 / 5$ provide interactions to a neighbouring molecules 14 via a network of $\mathrm{C}-\mathrm{H} \cdots \mathrm{X}(\mathrm{X}=\mathrm{Cl}, \mathrm{O})$ contacts (Figure 6.3). However, H-Atoms from neighbouring $\left[\mathrm{Mn}_{2}{ }_{2} \mathrm{Ni}_{3}{ }_{3} \mathrm{Cl}_{2}\left(\mathrm{~L}^{9}\right)_{4}\left(\mathrm{HL}^{9}\right)_{2}\left(\mathrm{H}_{2} \mathrm{O}\right)_{2}\right]$ molecules are not too far away. The closest contact between the manganese bound chlorine atom and the next $\mathrm{H}$-atom of a $\left[\mathrm{Mn}_{2}^{\mathrm{III}}{ }_{2} \mathrm{Ni}_{3} \mathrm{Cl}_{2}\left(\mathrm{~L}^{9}\right)_{4}\left(\mathrm{HL}^{9}\right)_{2}\left(\mathrm{H}_{2} \mathrm{O}\right)_{2}\right]$ moiety is $3.013 \AA(\mathrm{Cl1} \cdots \mathrm{H} 41-\mathrm{C} 41[2-x, 2-y, 2-$ $z]$ ). The latter belongs to a pyrazol-group. For $14 \cdot 6 \mathrm{C}_{2} \mathrm{H}_{4} \mathrm{Cl}_{2}$ a direct molecular contact via the manganese bound clorine atom to a hydrogen atom (H41) of a neigbouring $\left[\mathrm{Mn}_{2}^{\mathrm{III}}{ }_{2} \mathrm{Ni}_{3}{ }_{3} \mathrm{Cl}_{2}\left(\mathrm{~L}^{9}\right)_{4}\left(\mathrm{HL}^{9}\right)_{2}\left(\mathrm{H}_{2} \mathrm{O}\right)_{2}\right]$ molecule can be found. Other intermolecular contacts via solvent molecules are also present (Figure 6.4). The disordered $\mathrm{C}_{2} \mathrm{H}_{4} \mathrm{Cl}_{2}$ solvent molecule containing the atoms $\mathrm{Cl} 6 / 7$ also acts as a bridge with close $\mathrm{C}-\mathrm{H} \cdots \mathrm{X}$ contacts between two $\left[\mathrm{Mn}_{2}^{\mathrm{III}} \mathrm{Ni}_{3}{ }_{3} \mathrm{Cl}_{2}\left(\mathrm{~L}^{9}\right)_{4}\left(\mathrm{HL}^{9}\right)_{2}\left(\mathrm{H}_{2} \mathrm{O}\right)_{2}\right]$ units. This interaction is not shown in Figure 6.4. In case of 15. $8 \mathrm{CH}_{2} \mathrm{Cl}_{2}$ two short contacts of the bromine atom of one $\left[\mathrm{Mn}_{2}{ }_{2} \mathrm{Ni}_{3}{ }_{3} \mathrm{Br}_{2}\left(\mathrm{~L}^{9}\right)_{4}\left(\mathrm{HL}^{9}\right)_{2}\left(\mathrm{H}_{2} \mathrm{O}\right)_{2}\right]$ to neighbouring hydrogen atoms $\mathrm{H}-\mathrm{C}(\mathrm{H} 31 \mathrm{~A}$ and $\mathrm{H} 41)$ are present, achieving a direct contact to the next $\left[\mathrm{Mn}^{\mathrm{III}}{ }_{2} \mathrm{Ni}_{3}{ }_{3} \mathrm{Br}_{2}\left(\mathrm{~L}^{9}\right)_{4}\left(\mathrm{HL}^{9}\right)_{2}\left(\mathrm{H}_{2} \mathrm{O}\right)_{2}\right]$ molecules. Additional intermolecular contacts via solvent molecules containing $\mathrm{Cl} 2 / \mathrm{Cl} 3$ are present (Figure 6.5).

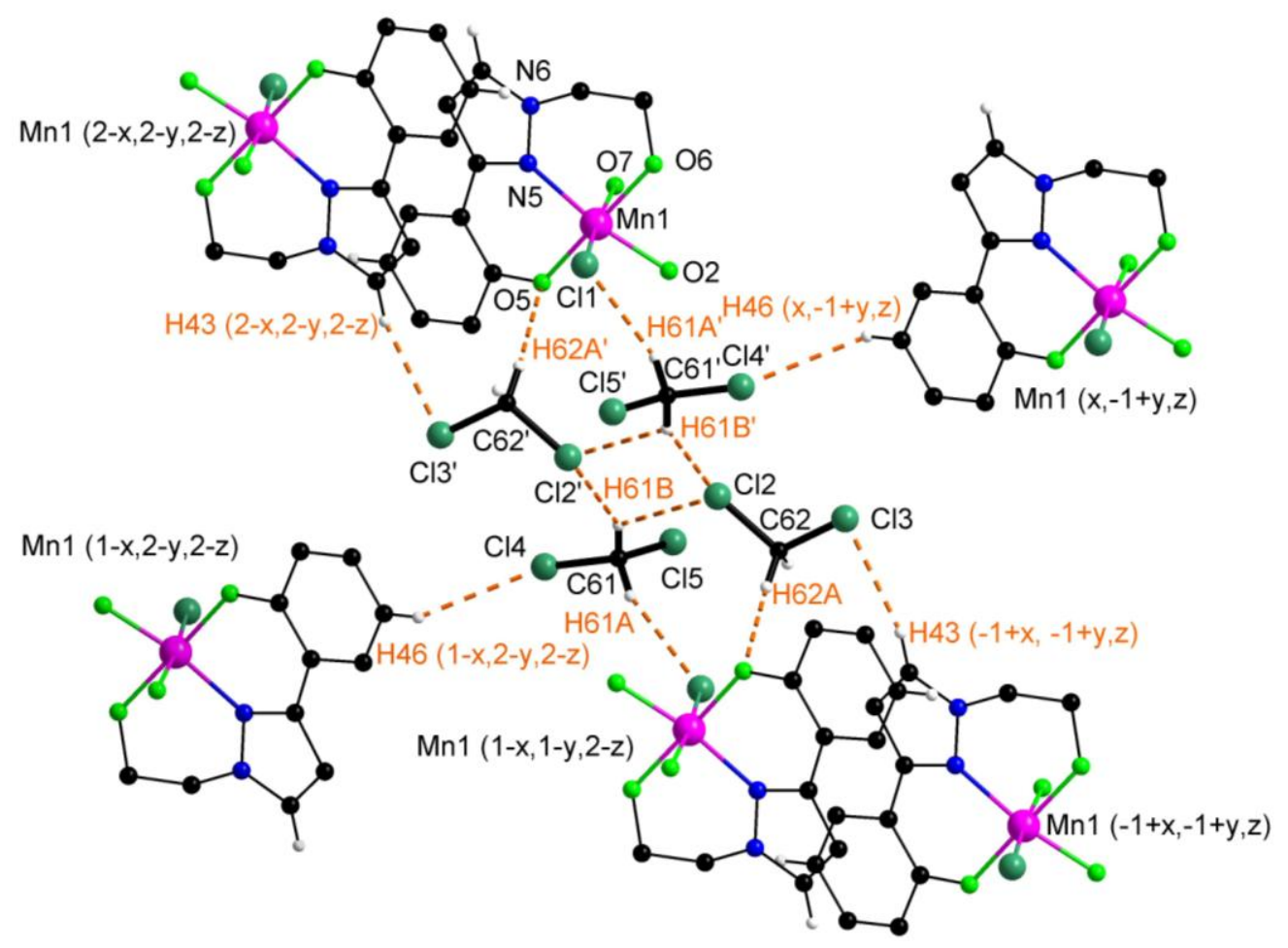

Figure 6.3. Emphasis of some intermolecular $\mathrm{C}-\mathrm{H} \cdots \mathrm{X}$ contacts in $\mathbf{1 4} \cdot 8 \mathrm{CH}_{2} \mathrm{Cl}_{2}$. Symmetry transformation used to generate equivalent atoms: (') $1-x, 1-y, 2-z$. Other symmetry transformations are given in the figure. 


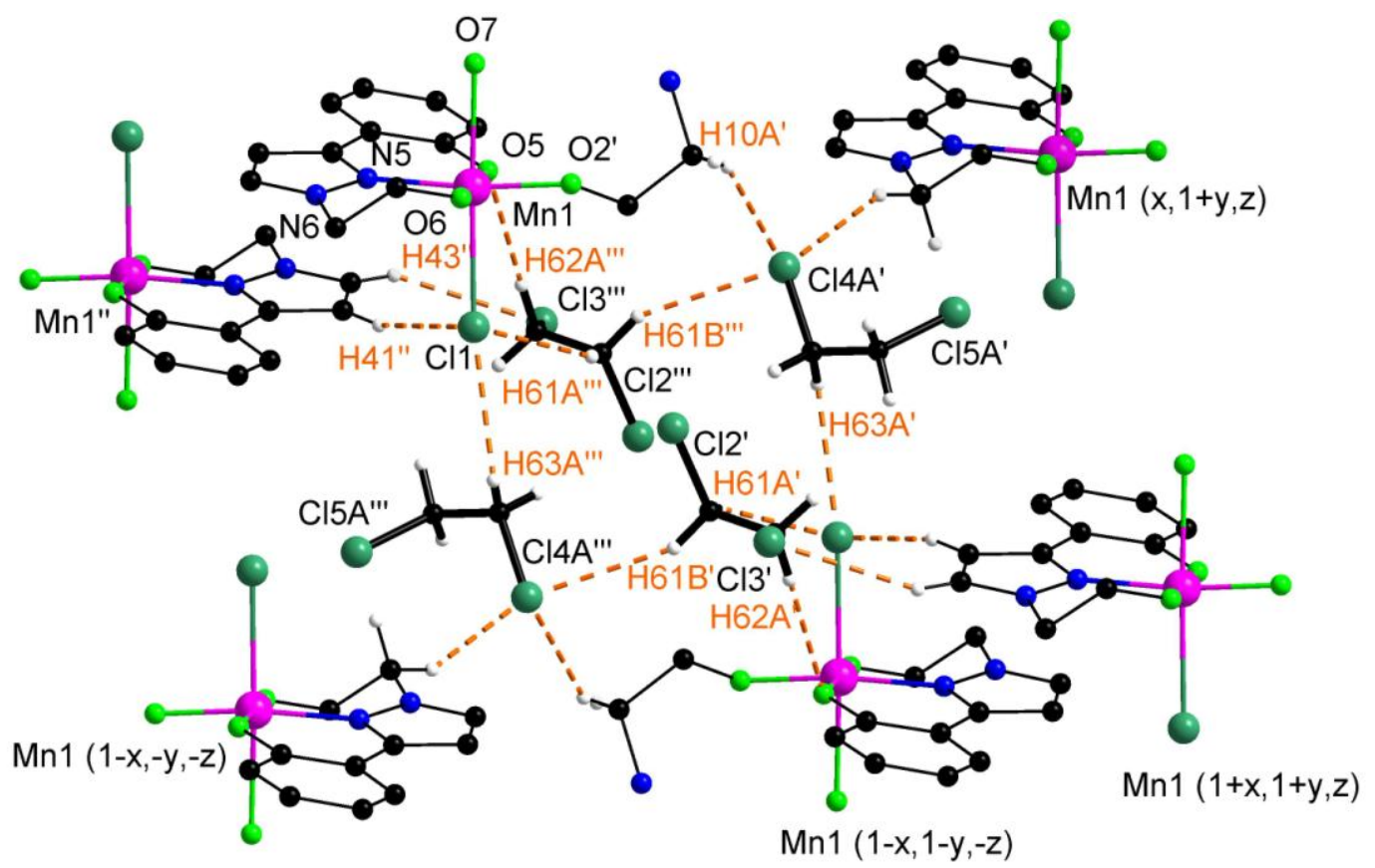

Figure 6.4. Emphasis of some intermolecular $\mathrm{C}-\mathrm{H} \cdots \mathrm{X}$ contacts in $\mathbf{1 4} \cdot 6 \mathrm{C}_{2} \mathrm{H}_{4} \mathrm{Cl}_{2}$. Symmetry transformations used to generate equivalent atoms: (') $1-x, 1-y, 1-z$, (') $-x,-y,-z$, (',') $x, y,-1+z$. Other symmetry transformations are given in the figure.

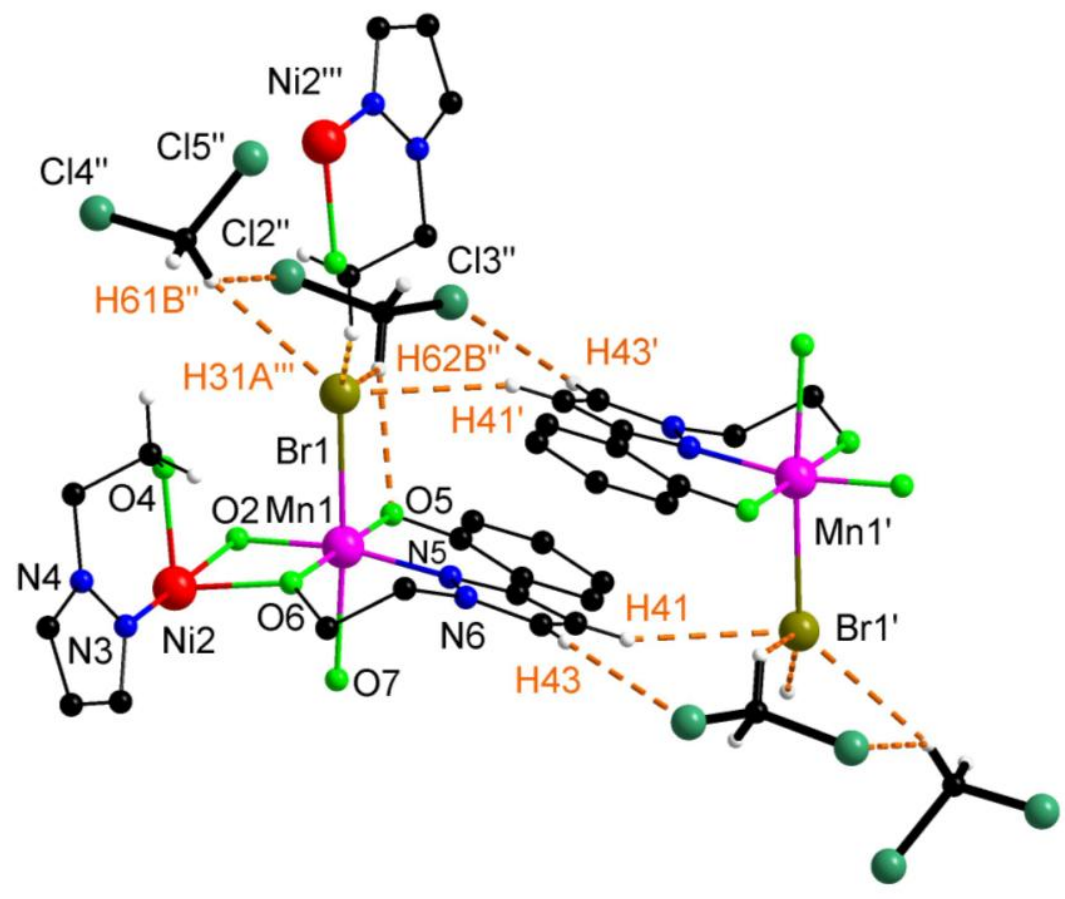

Figure 6.5. Emphasis of some intermolecular $\mathrm{C}-\mathrm{H} \cdots \mathrm{X}$ contacts in $\mathbf{1 5} \cdot 8 \mathrm{CH}_{2} \mathrm{Cl}_{2}$. Symmetry transformations used to generate equivalent atoms: (') $2-x, 2-y, 2-z$, (') $1-x, 1-y, 2-z$, (',') $2-x, 1-y, 2-z$. 
It seems that in all compounds at least one close intermolecular contact between two $\left[\mathrm{Mn}_{2}{ }_{2} \mathrm{Ni}_{3}{ }_{3} \mathrm{X}_{2}\left(\mathrm{~L}^{9}\right)_{4}\left(\mathrm{HL}^{9}\right)_{2}\left(\mathrm{H}_{2} \mathrm{O}\right)_{2}\right]$ molecules is present between the manganese bound halide atom and the hydrogen atom of a neighbouring pyrazole group, namely H41. Since a center of inversion is present within each molecule a chain is formed within the crystal (Figure 6.6). However, one should keep in mind that this is only one of several interactions which in a whole build up a 3-dimensional network.

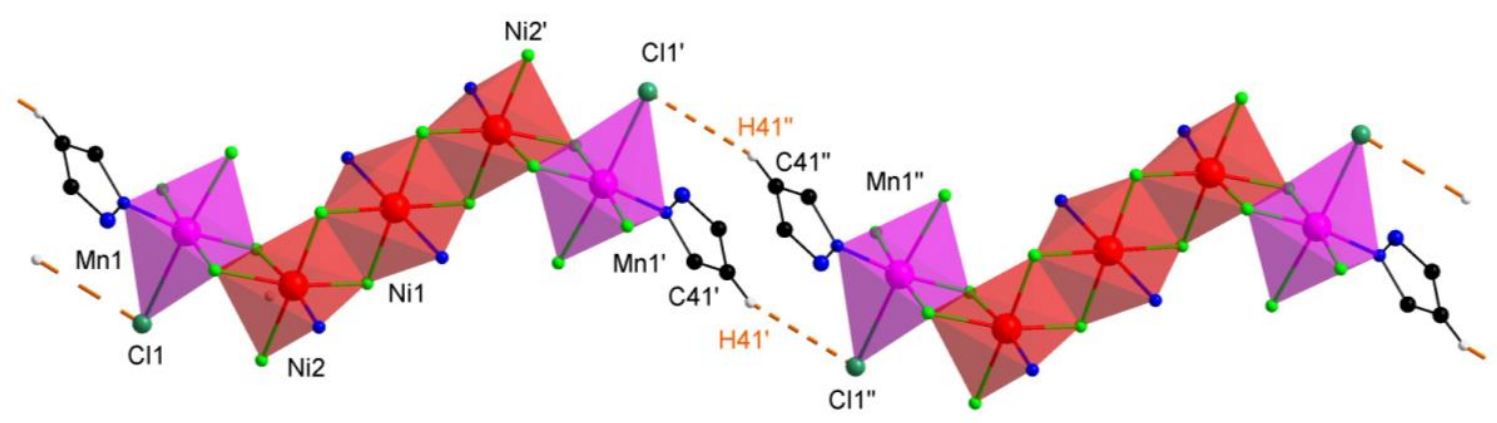

Figure 6.6. Emphasis of intermolecular $\mathrm{C}-\mathrm{H} \cdots \mathrm{Cl}$ contacts in $\mathbf{1 4} \cdot 8 \mathrm{CH}_{2} \mathrm{Cl}_{2}$. Symmetry transformations used to generate equivalent atoms: (') $1-x, 1-y, 1-z$, (') $-1+x,-1+y,-1+z$. 


\subsection{Magnetic properties}

Magnetic properties of $14 \cdot 8 \mathrm{CH}_{2} \mathrm{Cl}_{2}$ and $15 \cdot 8 \mathrm{CH}_{2} \mathrm{Cl}_{2}$ were investigated using a commercial SQUID MPMS system as well as a home-made micro-Hall-bar magnetometer. ${ }^{77} \mathrm{We}$ have performed susceptibility measurements of a powder sample of $\mathbf{1 4} \cdot 8 \mathrm{CH}_{2} \mathrm{Cl}_{2}$ (Figure 6.7 ; left) and $15 \cdot 8 \mathrm{CH}_{2} \mathrm{Cl}_{2}$ (Figure 6.7; right) using the SQUID magnetometer. The measurements were done at magnetic field values of $0.5,1.0$, and $5 \mathrm{~T}$ in a temperature range between 2 and 300 $\mathrm{K}$.
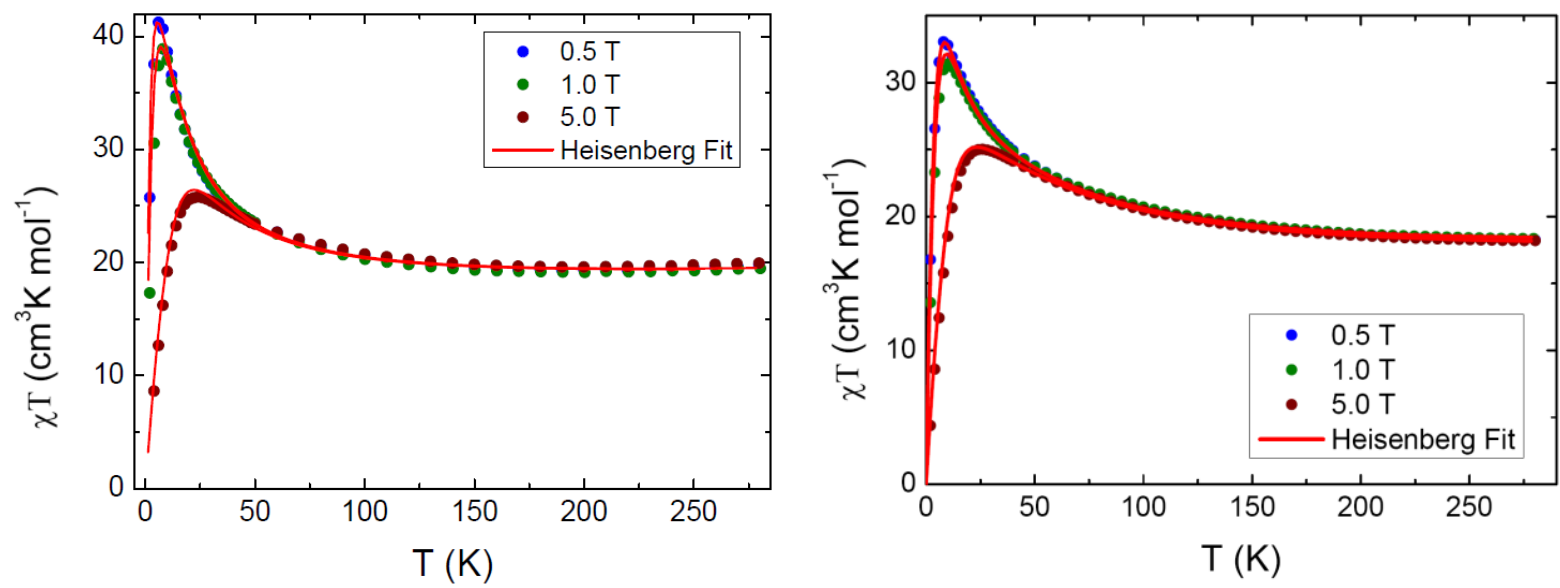

Figure 6.7. Susceptibility vs. temperature of a microcrystalline sample of $\mathbf{1 4}$ (in $\mathbf{1 4} \cdot 8 \mathrm{CH}_{2} \mathrm{Cl}_{2}$ ) (left) and 15 (in 15. $8 \mathrm{CH}_{2} \mathrm{Cl}_{2}$ ) (right). The various fields are indicated in the inset. The straight line is a Heisenberg fit to the data (see text).

The observed $\chi_{\mathrm{M}} T$ value (considering dimer) at room temperature is $20 \mathrm{~cm}^{3} \mathrm{Kmol}^{-1}$ (corresponding to an effective moment $\mu_{\text {eff }}=12.65 \mu_{\mathrm{B}}$ ) for $\mathbf{1 4}$, and $18.00 \mathrm{~cm}^{3} \mathrm{Kmol}^{-1}$ (corresponding to an effective moment $\mu_{\mathrm{eff}}=12.00 \mu_{\mathrm{B}}$ ) for $\mathbf{1 5}$, those are consistent with the theoretical value expected for dimer of two uncoupled manganese(III), and three uncoupled nickel(II) ions (18.52 $\mathrm{cm}^{3} \mathrm{Kmol}^{-1}$ or $\mu_{\mathrm{eff}}=12.18 \mu_{\mathrm{B}}$ for $\left.g=2.03\right)$. In both cases, upon lowering the temperature, the $\chi_{\mathrm{M}} T$ value is nearly temperature independent up to $150 \mathrm{~K}$ for $\mathbf{1 4}$, and 100 $\mathrm{K}$ for $\mathbf{1 5}$, and then increases gradually to reach $42 \mathrm{~cm}^{3} \mathrm{Kmol}^{-1}$ (corresponding to an effective moment $\mu_{\text {eff }}=18.34 \mu_{\mathrm{B}}$ ) at $8 \mathrm{~K}$ for 14 and $37.5 \mathrm{~cm}^{3} \mathrm{Kmol}^{-1}$ (corresponding to an effective moment $\left.\mu_{\mathrm{eff}}=17.33 \mu_{\mathrm{B}}\right)$ at $8 \mathrm{~K}$ for $\mathbf{1 5}$. This behavior is consistent with overall intramolecular ferromagnetic coupling between the two manganese(III) and three nickel(II) ions and an $S_{\mathrm{T}}=$ 7 for both cases. 
Experimental data were simulated using a fitting procedure to the appropriate HeisenbergDirac-van-Vleck (HDvV) spin Hamiltonian for isotropic exchange coupling (eq. 6.1) for complexes 14-15.

$$
H_{\text {int }}=-J_{1}\left(S_{M 1} S_{N 1}+S_{M 2} S_{N 3}\right)-J_{2}\left(S_{N 1} S_{N 2}+S_{N 2} S_{N 3}\right)
$$

Where $J_{1}$ is the $\mathrm{Mn}-\mathrm{Ni}$ exchange coupling parameter and $J_{2}$ is the $\mathrm{Ni}-\mathrm{Ni}$ exchange coupling parameter.

From the numerically calculated best fit, shown with red lines in Figure 6.8, we obtained $J_{1} \approx$ $18 \mathrm{~K}$ and $J_{2} \approx 12 \mathrm{~K}$ with a $g$-factor $g=2.1$ for 14 , and $J_{1} \approx 42 \mathrm{~K}$ and $J_{2} \approx 18 \mathrm{~K}$ with a $g$-factor $g=2.1$ for 15 . This leads to the conclusion that we deal with a purely ferromagnetically coupled system with a ground-state spin of $S_{\mathrm{T}}=7$ in both cases.
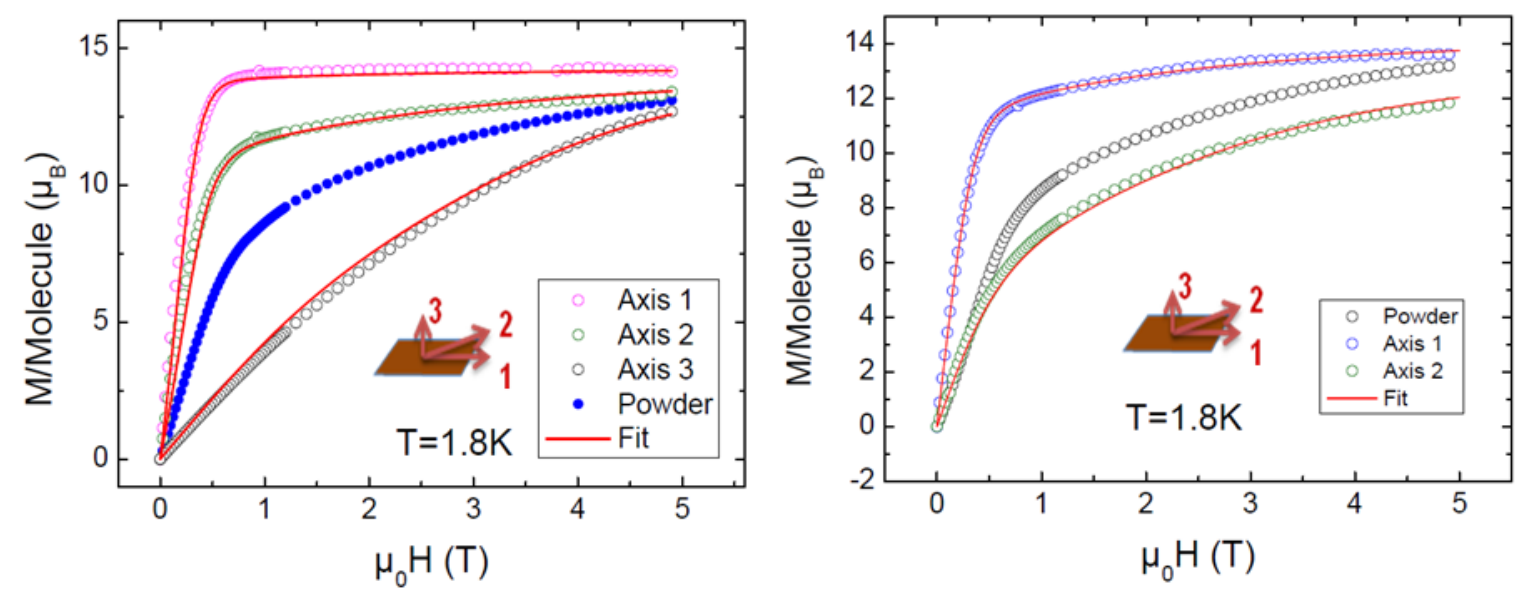

Figure 6.8. Magnetization versus field data for different orientations of a single crystal of $14 \cdot 8 \mathrm{CH}_{2} \mathrm{Cl}_{2}(\mathrm{left})$ and $\mathbf{1 5} \cdot 8 \mathrm{CH}_{2} \mathrm{Cl}_{2}$ (right) at $1.8 \mathrm{~K}$ and the corresponding powder data. The red lines represent best fits. The inset shows a top view of a typical crystal. Axis 1,2 and 3 are the different orientations indicated by the sketch in the inset.

Variable-field magnetization measurements for a polycrystalline sample and for a single crystal with different field orientations of $14 \cdot 8 \mathrm{CH}_{2} \mathrm{Cl}_{2}$ and $15 \cdot 8 \mathrm{CH}_{2} \mathrm{Cl}_{2}$ are depicted in Figure 6.8. While the magnetization of the powder sample and of the single-crystal sample at orientations axis 2 and 3 for $\mathbf{1 4} \cdot 8 \mathrm{CH}_{2} \mathrm{Cl}_{2}$ or axis 2 for $\mathbf{1 5} \cdot 8 \mathrm{CH}_{2} \mathrm{Cl}_{2}$ is not saturated at a field of $5 \mathrm{~T}$, the crystal oriented along axis 1 saturates already at about $0.6 \mathrm{~T}$. From the saturation value $M_{\mathrm{S}} \approx 14.2 \mu_{\mathrm{B}}$ for $\mathbf{1 4} \cdot 8 \mathrm{CH}_{2} \mathrm{Cl}_{2}$ and $M_{\mathrm{S}} \approx 13.9 \mu_{\mathrm{B}}$ for $15 \cdot 8 \mathrm{CH}_{2} \mathrm{Cl}_{2}$ one can also conclude a spin ground state of $S_{\mathrm{T}}=7$ in both cases, assuming a $g$-factor of 2.03 . These values are consistent with the values obtained from the powder susceptibility data. 
Since the ground-state multiplet is sufficiently well separated from the first exited multiplet, a giant spin Hamiltonian of the form

$$
H=D\left(S_{z}^{2}-\frac{1}{3} S(S+1)\right)+E\left(S_{x}^{2}-S_{y}^{2}\right)
$$

can be applied to the single crystal measurements depicted in Figure 6.8. From a numerical fit of this equation to the data, we were able to obtain estimation for the uniaxial anisotropy parameter $D \approx-0.5 \mathrm{~K}$ for $14 \cdot 8 \mathrm{CH}_{2} \mathrm{Cl}_{2}$ and $D \approx-0.45 \mathrm{~K}$ for $15 \cdot 8 \mathrm{CH}_{2} \mathrm{Cl}_{2}$. Within this framework, one can get an estimation for the effective energy barrier for spin reversal of $U_{\text {eff }}=|D| S^{2} \approx 24.5 \mathrm{~K}$ for $14 \cdot 8 \mathrm{CH}_{2} \mathrm{Cl}_{2}$ and $22.05 \mathrm{~K}$ for for $15 \cdot 8 \mathrm{CH}_{2} \mathrm{Cl}_{2}$. Unfortunately, we were unable to determine a reliable value of the $E$ parameter with this method, as the sample crystals were rather small $(\approx 20 \mu \mathrm{g})$ and very thin. Furthermore, the softness of the crystal made it almost impossible to align them with the necessary angular precision.

To probe the presence of slow dynamics in these molecular systems and thus the presence of SMM behavior, ac susceptibility measurements were performed systematically on these compounds. The measurements were made in the frequency range 10-1488 $\mathrm{Hz}$ and temperature between 1.8 and $4.25 \mathrm{~K}$. Figure 6.9 and 6.10 shows the results of the ac-magnetic susceptibility measurements for $14 \cdot 8 \mathrm{CH}_{2} \mathrm{Cl}_{2}$ and $15 \cdot 8 \mathrm{CH}_{2} \mathrm{Cl}_{2}$ respectively as the plots of $m^{\prime}$ versus $T$, and $m^{\prime \prime}$ versus $T$. In both cases below circa $3 \mathrm{~K}$, a frequency-dependent decreases in the in-phase ( $m$ ) signal (Figure 6.9; left and Figure 6.10; left) and a concomitant increases in the out-of-phase $\left(\mathrm{m}^{\prime}\right)$ signal (Figure 6.9; right and Figure 6.10; right) were observed. This behavior is indicative of the slow relaxation of the magnetization, which is unusual for a paramagnet without an applied field. Moreover, in AC-external magnetic field, frequency dependence of real $\left(m^{\prime}\right)$ and imaginary $\left(m^{\prime \prime}\right)$ parts of $m$ can be plot, which known as a ColeCole or Argand plot (inset in Figure 6.9; right for $\mathbf{1 4} \cdot 8 \mathrm{CH}_{2} \mathrm{Cl}_{2}$, and inset in Figure 6.10; right for $15 \cdot 8 \mathrm{CH}_{2} \mathrm{Cl}_{2}$ ). At a fixed temperature the almost perfect semi-circle of the cole-cole plot in both complexes is a clear evidence of a relaxation process with a single relaxation time $\tau_{0}$. Thus such kind of behavior suggest that both complexes might be a new family of SMMs, but do not confirm that the both complexes are SMMs, as intermolecular interactions and phonon bottlenecks can have similar AC susceptibility responses. ${ }^{78}$ 

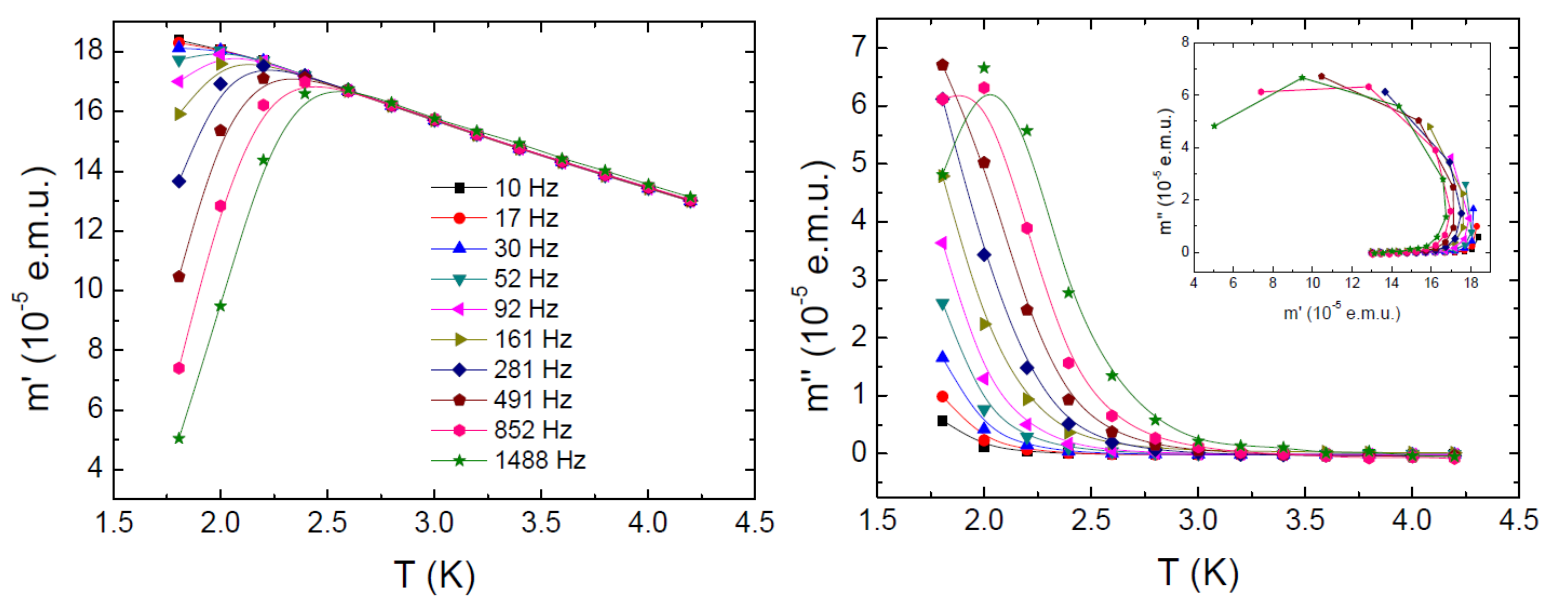

Figure 6.9. Plots of in-phase $\left(m^{\prime}\right.$, left $)$ and out-of-phase $\left(m^{\prime \prime}\right.$, right) ac susceptibility versus temperature for a microcrystalline sample of $\mathbf{1 4} \cdot 8 \mathrm{CH}_{2} \mathrm{Cl}_{2}$. The data were collected in an ac field of $2.56 \mathrm{Oe}$ oscillating at the indicated frequencies. The inset in the lower Figure shows a Cole-Cole plot ( $m^{\prime \prime}$ versus $\left.m^{\prime}\right)$.
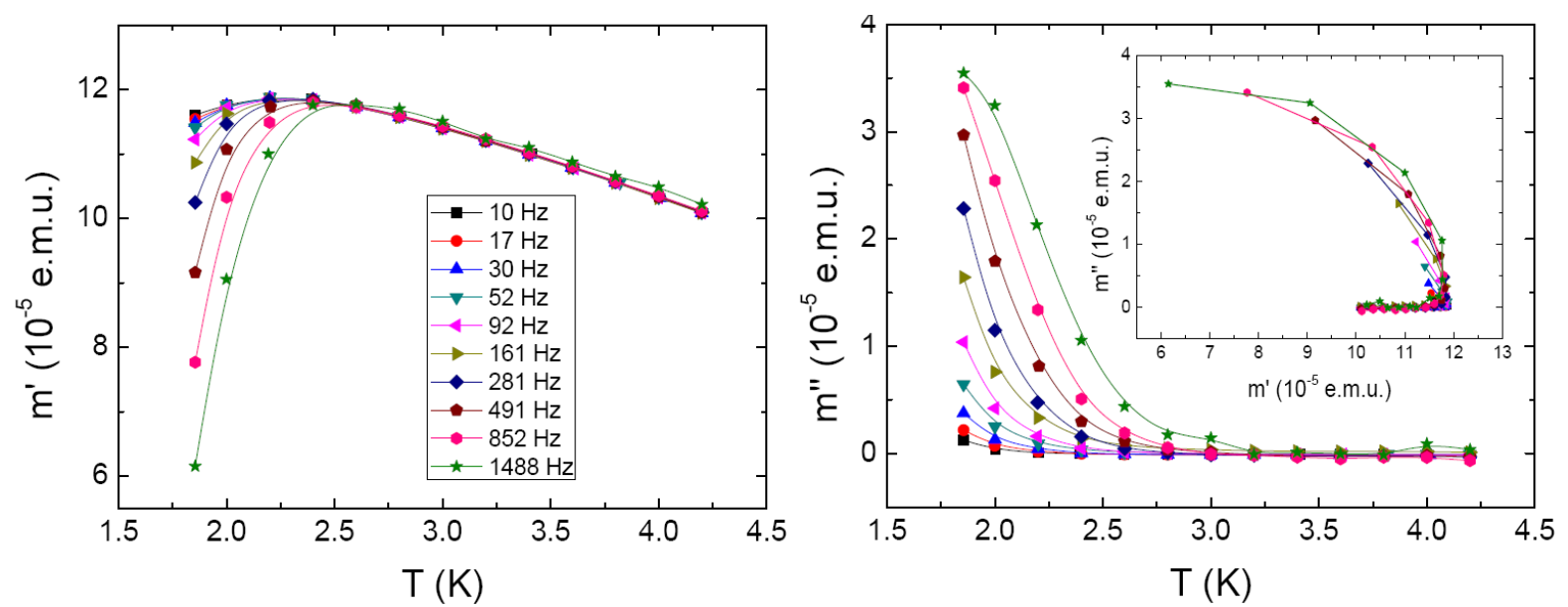

Figure 6.10. Plots of in-phase $\left(m^{\prime}\right.$, left $)$ and out-of-phase ( $m^{\prime \prime}$, right) ac susceptibility versus temperature for a microcrystalline sample of $\mathbf{1 5} \cdot 8 \mathrm{CH}_{2} \mathrm{Cl}_{2}$. The data were collected in an ac field of $2.56 \mathrm{Oe}$ oscillating at the indicated frequencies. The inset in the lower Figure shows a Cole-Cole plot ( $m^{\prime \prime}$ versus $m$ ).

Confirmation was therefore sought by magentisation versus DC field scans on single crystals of $14 \cdot 8 \mathrm{CH}_{2} \mathrm{Cl}_{2}$ and $15 \cdot 8 \mathrm{CH}_{2} \mathrm{Cl}_{2}$ using an array of micro-Hall-bar magnetometer. This magnetometer is equipped with several $10 \times 10 \mu \mathrm{m}^{2}$ Hall bars. Micrometer-sized single crystals of the sample were placed on top of the active area of the Hall bars. The crystals were oriented with the easy axis approximately parallel to the applied magnetic field. The measurements were performed at several temperatures in the range of $0.3-2.0 \mathrm{~K}$, and sweep rates in the range of $12 \mathrm{mTs}^{-1}-200 \mathrm{mTs}^{-1}$. 

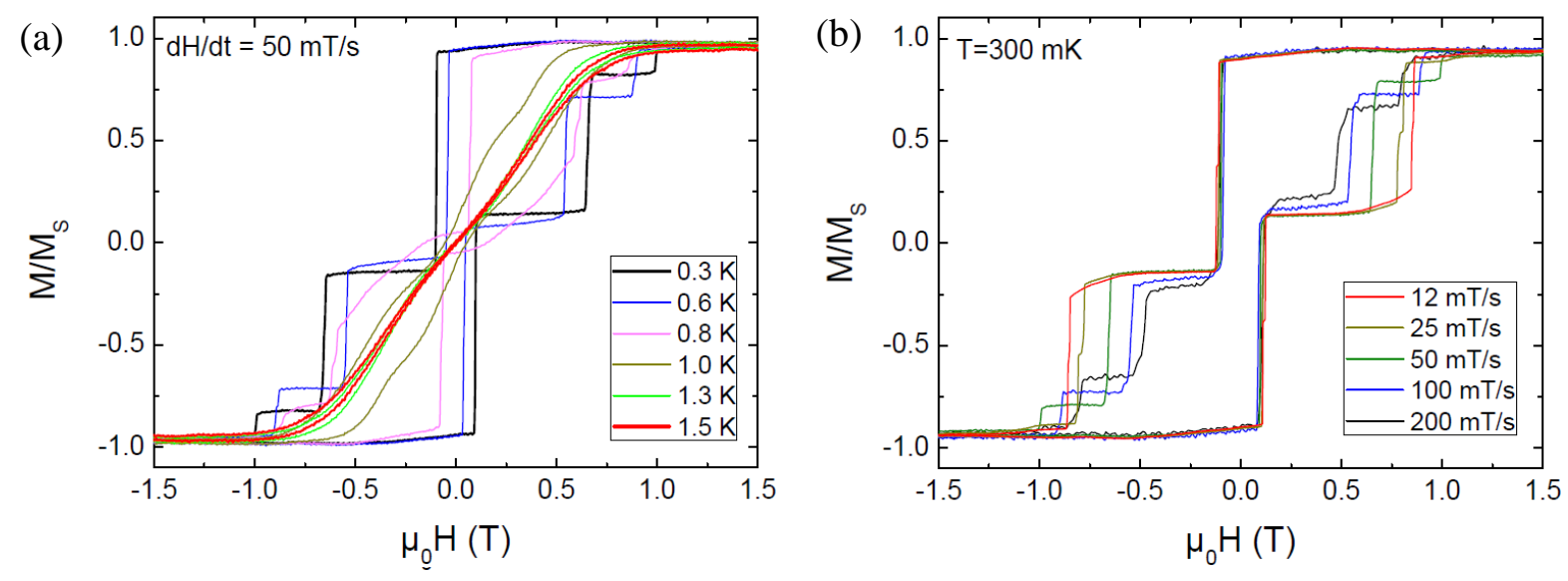

Figure 6.11. Magnetisation $(M)$ versus dc field hysteresis loops for a single crystal of $\mathbf{1 4} \cdot 8 \mathrm{CH}_{2} \mathrm{Cl}_{2}$ at constant sweep rate and variable temperature (a) and constant temperature and variable sweep rate (b).
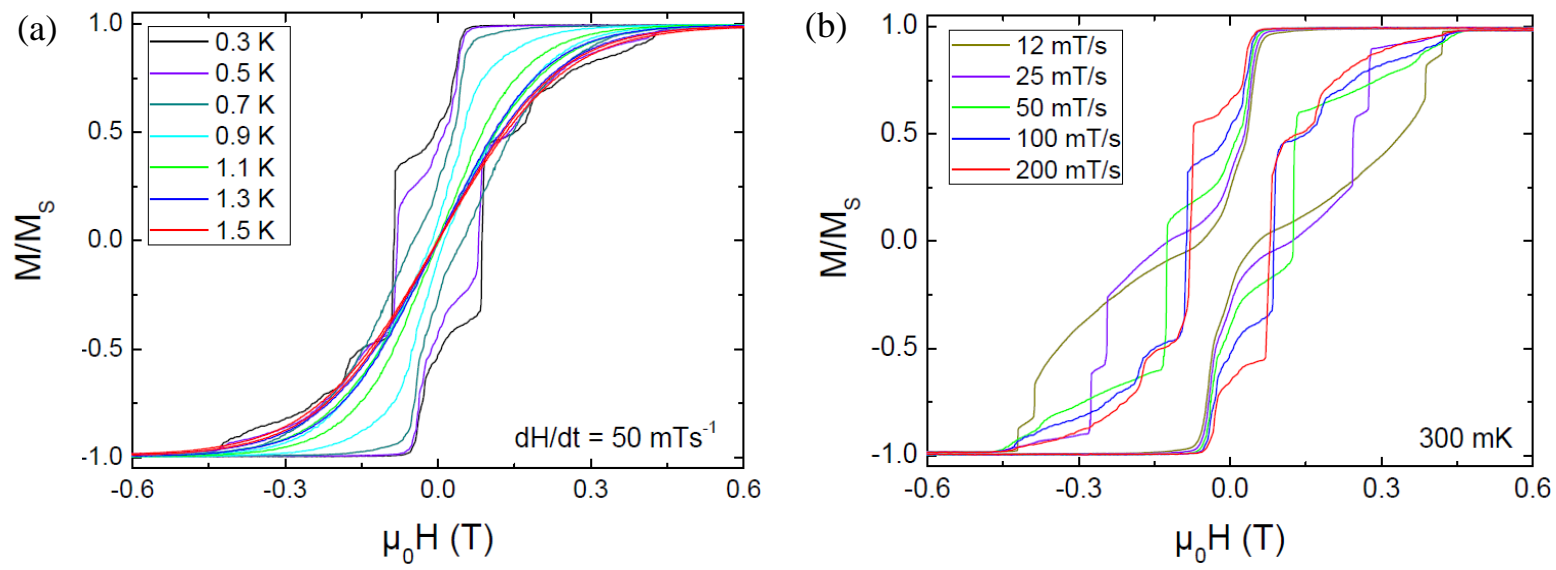

Figure 6.12. Magnetisation $(M)$ versus dc field hysteresis loops for a single crystal of $\mathbf{1 5} \cdot 8 \mathrm{CH}_{2} \mathrm{Cl}_{2}$ at constant sweep rate and variable temperature (a) and constant temperature and variable sweep rate (b).

Magnetization hysteresis loops were observed below circa $1.5 \mathrm{~K}$ for both compound, at which point the coercivity increases with decreasing temperature and increasing field sweep rate (Figure 6.11 for $\mathbf{1 4} \cdot 8 \mathrm{CH}_{2} \mathrm{Cl}_{2}$, and Figure 6.12 for $\mathbf{1 5} \cdot 8 \mathrm{CH}_{2} \mathrm{Cl}_{2}$ ), as expected for an $\mathrm{SMM}$ below its blocking temperature $\left(T_{\mathrm{B}}\right)$. Compound $14 \cdot 8 \mathrm{CH}_{2} \mathrm{Cl}_{2}$ and $15 \cdot 8 \mathrm{CH}_{2} \mathrm{Cl}_{2}$ are thus new SMMs. The surprising features in Figure 6.11 and Figure 6.12 are the highly resolved QTM steps at periodic field positions. Although both compounds have similar model parameters and show sharp QTM steps, the overall shape of the hysteresis loops looks different and is mainly determined by relaxation paths caused by intermolecular interactions. At the moment, it is not possible to accurately simulate the discussed 3D-like intermolecular coupling scheme because the dimension of the resulting Hamiltonian exceeds the memory capacity of currently 
available computers. In the case of $\mathbf{1 4} \cdot 8 \mathrm{CH}_{2} \mathrm{Cl}_{2}$ the first relaxation step in the hysteresis loop is above the zero crossing of the magnetic field, while in the case of $15 \cdot 8 \mathrm{CH}_{2} \mathrm{Cl}_{2}$ it is below the zero crossing. This can be understood by assuming intermolecular ferromagnetic interaction in case of $\mathbf{1 4} \cdot 8 \mathrm{CH}_{2} \mathrm{Cl}_{2}$ and antiferromagnetic interaction in case of $15 \cdot 8 \mathrm{CH}_{2} \mathrm{Cl}_{2}$. ${ }^{70 b}$ It can be due to different magnetic dipolar interaction between the clusters of both compounds (intermolecular distance between $\mathrm{Mn} \cdots \mathrm{Mn}$ is $7.56 \AA$ for $\mathbf{1 4} \cdot 8 \mathrm{CH}_{2} \mathrm{Cl}_{2}, 7.72 \AA$ for $15 \cdot 8 \mathrm{CH}_{2} \mathrm{Cl}_{2}$ ). Thus weak ferro and antiferromagnetic intermolecular interaction in the crystal lattice gives a significant effect on the manifestation of slow relaxation of magnetisation. Hence these complexes represent a new family of "exchange-biased single-molecule magnets", where the exchange bias can be controlled by chemical and structural modifications.

\subsection{HF-ESR Spectroscopy}

High field electron spin resonance (HF-ESR) has an improtant tool to unambiguously detremine the spin of the ground states as well as sign and magnitude of the axial ZFS parameter $D$. The measurements were performed on an oriented powder sample of 14. $8 \mathrm{CH}_{2} \mathrm{Cl}_{2}$ in magnetic fields up to $15 \mathrm{~T}$. Owing to a strong magnetic anisotropy (see below) the single crystalline powder particles in the sample were aligned in the magnetic field along their crystalline magnetic anisotropy axis. A typical ESR spectrum at a frequency $v$ of 332 $\mathrm{GHz}$ and $T=20 \mathrm{~K}$ is shown in Figure 6.13. The spectrum possesses a relatively simple structure consisting of seven well defined almost equally spaced absorption lines with a separation of the resonance fields $B_{\text {res }}$ by $\sim 1 \mathrm{~T}$. The intensity of the lines continuously decreases with increasing the magnetic field strength. Measurements at different excitation frequencies reveal a linear relationship between $v$ and $B_{\text {res }}$ for each absorption line. The respective $v$ vs. $B_{\text {res }}$ dependencies (resonance branches) are plotted together in Figure 6.13. The slope $\mathrm{d} v / \mathrm{d} B$ of all resonance branches is practically the same. This enables a straightforward determination of the $g$-factor $g=\left(h / \mu_{\mathrm{B}}\right) \mathrm{d} v / \mathrm{d} B$ which for $\mathbf{1 4} \cdot 8 \mathrm{CH}_{2} \mathrm{Cl}_{2}$ amounts to $g=2.09$. Here $h$ is the Planck constant and $\mu_{\mathrm{B}}$ is the Bohr magneton. Note a sizable deviation of the $g$-factor from the value of 2 characteristic of $\mathrm{Mn}$ (III) ions. It is obviously due to the contribution of $\mathrm{Ni}$ (II) species to the total $g$-factor of the complex since $\mathrm{Ni}$ (II) ions usually possess $g$ values in the range $2.2-2.3 .{ }^{79}$ As shown in Figure 6.13 the extrapolation of the $v\left(B_{\text {res }}\right)$-dependence of the leftmost line in the ESR spectrum (line 1) to zero magnetic field yields the magnitude of the magnetic anisotropy gap $\Delta$ is amounting to $150 \mathrm{GHz}(7.2 \mathrm{~K})$. Since $\Delta$ is related with the anisotropy parameter $D_{\text {mol }}$ of the molecular complex as $\left|D_{\text {mol }}\right|=$ 
$\Delta /\left(S_{\text {tot }}^{2}-\left(S_{\text {tot }}-1\right)^{2}\right)$, where $S_{\text {tot }}$ is the total spin of the complex, with $S_{\text {tot }}=7$ one obtains the absolute value of $D_{\text {mol }}$ for $\mathbf{1 4} \cdot 8 \mathrm{CH}_{2} \mathrm{Cl}_{2}\left|D_{\mathrm{mol}}\right|=11 \mathrm{GHz}(0.55 \mathrm{~K}) .^{80,81}$

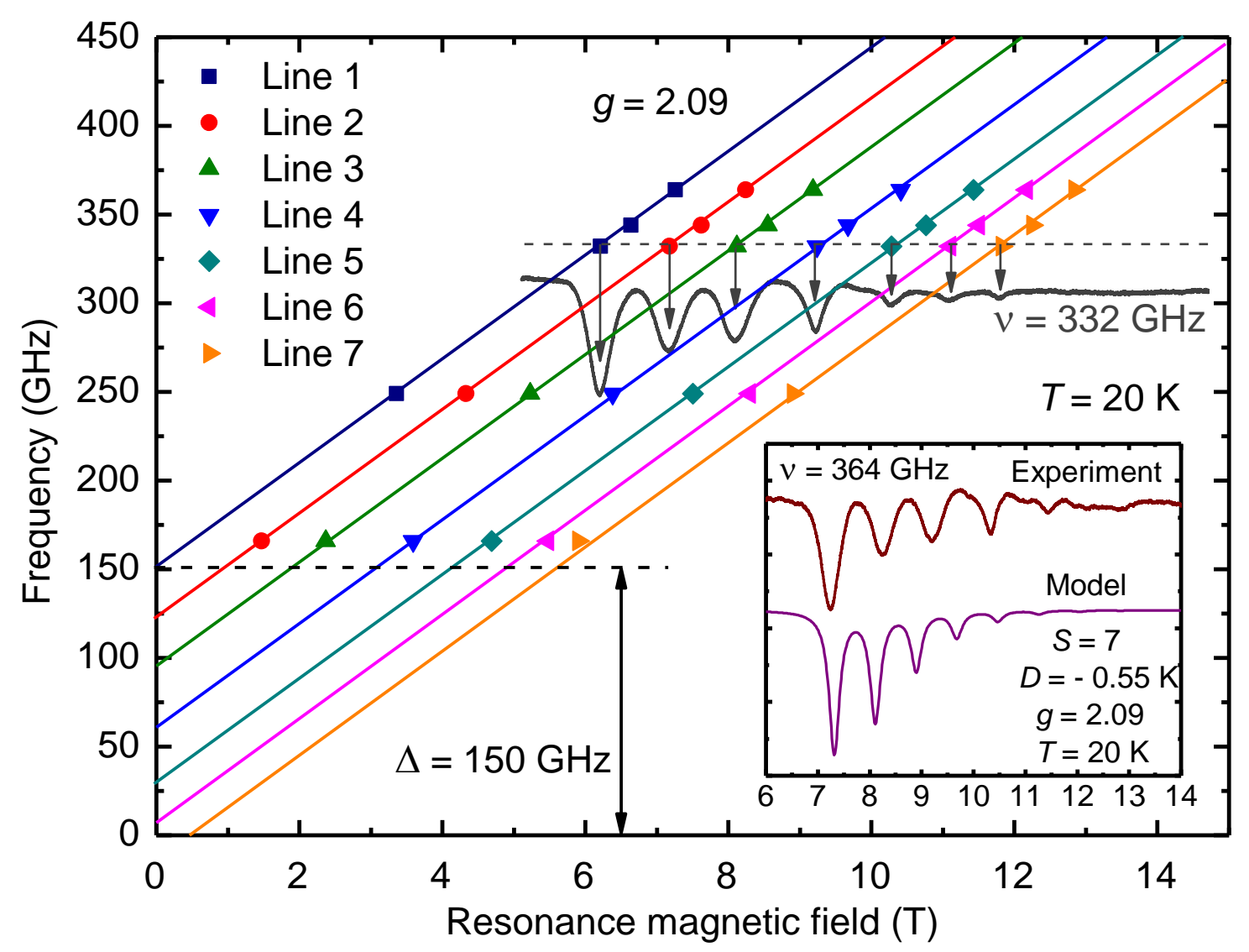

Figure 6.13. Frequency $v$ versus resonance magnetic field $B_{\text {res }}$ dependence of the ESR lines (symbols) and a representative ESR spectrum of $\mathbf{1 4} \cdot 8 \mathrm{CH}_{2} \mathrm{Cl}_{2}$ at $T=20 \mathrm{~K}$. Solid lines are linear fits to the experimental data points. Inset: Comparison of the experimental and modelled ESR spectra (see the text).

The sign of $D_{\text {mol }}$ can be unambiguously determined from the $T$-dependence of the ESR spectrum which is presented in Figure 6.14. Here, one observes a remarkable shift of the spectral weight to lower magnetic fields at low temperatures whereas the positions of the lines do not change. Such kind of the redistribution of the ESR spectral weight occurs in the case of a spin doublet ground state $\mid \pm S_{\text {tot }}{ }^{\mathrm{Z}}>$ corresponding to the negative sign of $D_{\mathrm{mol}}$, whereas for the case of a singlet state $|0\rangle$, i.e. for a positive sign of $D_{\text {mol }}$, the spectral weight should shift to higher fields. ${ }^{81}$ Thus the HF-ESR results give clear evidence for a bistable 'easy axis' ground state of the $14 \cdot 8 \mathrm{CH}_{2} \mathrm{Cl}_{2}$ complex with a substantial anisotropy barrier $U=S_{\text {tot }}{ }^{2}\left|D_{\text {mol }}\right|=27 \mathrm{~K}$ between two degenerate ground states $S_{\text {tot }}^{\mathrm{Z}}=+7$ and $S_{\text {tot }}^{\mathrm{Z}}=-7$.

Finally, with the above obtained parameters the ESR spectrum of $14 \cdot 8 \mathrm{CH}_{2} \mathrm{Cl}_{2}$ was modelled using the Easy Spin toolbox for Matlab ${ }^{82}$ for the case of the parallel orientation of the "easy 
axis' of the complex to the direction of the magnetic field, i.e. the situation which is expected for the oriented powder sample.

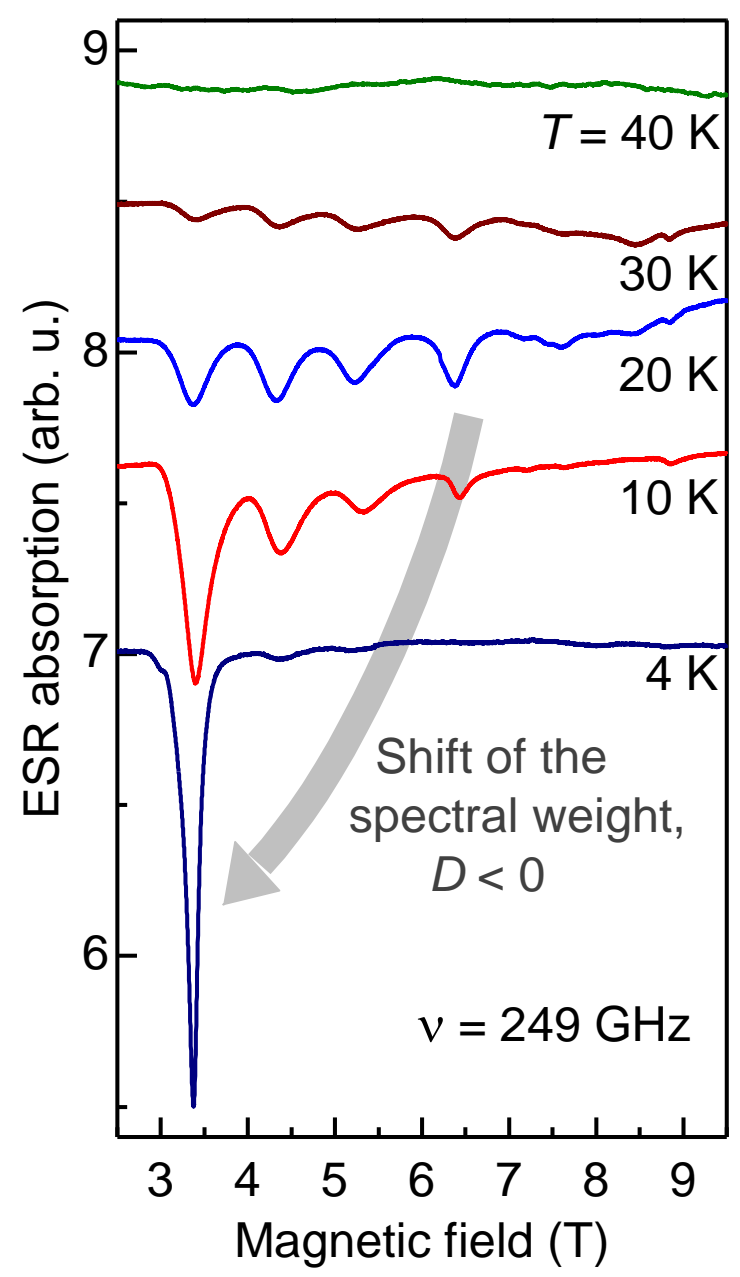

Figure 6.14. Temperature dependence of the ESR spectra of $\mathbf{1 4} \cdot 8 \mathrm{CH}_{2} \mathrm{Cl}_{2}$ at $v=249 \mathrm{GHz}$. Note a considerable shift of the spectral weight to low fields with decreasing the temperature indicative of a negative sign of the anisotropy parameter $D$ (see the text).

As can be seen in the inset of Figure 6.13 the simulation captures well the main features of the experimentally observed ESR spectrum: the extent of the fine structure of the spectrum, the number of lines and their relative intensities. The minor discrepancies between the model and the experiment could be due to an imperfect alignment of the powder particles as well as to the simplifications of the model. 


\subsection{Conclusions}

A new family of pentanuclear $\mathrm{Mn}_{2} \mathrm{Ni}_{3}$ compmexes have been synthesized and charecterised. These species posses a significant easy axis-type magnetoanistropy $(D \approx-0.45-0.55 \mathrm{~K})$ together with ferromagnetic intramolecular exchange interactions resulting in an $S_{\mathrm{T}}=7$ ground state. In combination, these two molecular properties give rise to an energy barrier $(\sim 22-27 \mathrm{~K})$ to thermal relaxation of the magnetisation. Magnetization versus direct-current field sweeps below $1.5 \mathrm{~K}$ revealed sweep rate dependent hysteresis loops with magnetization relaxation, definitely indicating that both complexes are single-molecule magnets that exhibit quantum tunnelling of magnetization (QTM) through an anisotropy barrier. Detailed magnetic measurements have revealed that relatively weak intermolecular interactions are manifest in both compounds. Despite the common molecular attributes, the intermolecular interactions were found to be ferromagnetic for $14 \cdot 8 \mathrm{CH}_{2} \mathrm{Cl}_{2}$ and antiferromagnetic for $15 \cdot 8 \mathrm{CH}_{2} \mathrm{Cl}_{2}$. The sign of the intermolecular exchange is related to the three dimensional arrangement of the clusters in the crystal, and the nature of the crystal packing is itself dependent on small chemical and structural changes to the molecules. Thus, these complexes represent a new family of "exchange-biased SMMs", where small chemical changes ultimately control the nature of the "exchange-bias". 


\section{Chapter 7}

\section{High Nuclearity Aggregate of Mixed Valent Manganese Clusters and Heteronuclear $\mathrm{Mn}^{\mathrm{III}}{ }_{2} \mathrm{Ln}_{2}^{\mathrm{III}}{ }_{2}$ SMMs: Synthesis, Structural Characterization, and Magnetic Properties}

\subsection{Introduction}

A bottom-up approach to the synthesis and crystallization of high-nuclearity, magnetically coupled transition-metal aggregates is currently a major focus of research in the area of nanoscale materials. ${ }^{83}$ In this field, nanoscale molecules exhibiting single-molecule magnet (SMM) behavior are of special interest owing to their potential applications in data storage and quantum computation as quantum bits. Manganese carboxylate aggregates have been a fruitful source of new SMMs in recent years ${ }^{84}$ because these clusters include the necessary ingredients of SMMs, i.e., large-spin ground states $(S)$ and a large uniaxial anisotropy $(D)$ (here arising from the presence of Jahn-Teller distorted $\mathrm{Mn}^{\mathrm{III}}$ ions), giving rise to the superparamagnetic-like property governed by an energy barrier in the form of $\Delta=|D| S_{\mathrm{T}}^{2}$ for integer spin and $|D|\left(S_{\mathrm{T}}{ }^{2}-1 / 4\right)$ for half-integer spin to magnetisation relaxation, although a recent reappraisal of this suggests that the relationship is linearly dependent on the spin $S_{\mathrm{T}}{ }^{85}$ in turn directing research toward increasing the anisotropy in new systems.

One approach is to synthesize high-nuclearity, mixed-valance manganese clusters via selfassembly techniques, in which the perfect alignment of the Jahn-Teller axes with the largest possible number of paramagnetic centres should be most advantageous for large $D$ value. Although there are many examples of mixed-valance $\mathrm{Mn}^{\mathrm{II}} / \mathrm{Mn}^{\mathrm{III}}$ clusters, and the $\mathrm{Mn}^{\mathrm{III}}$ centers induce a local anisotropy through Jahn-Teller distortions, only a few clusters possess a resultant anisotropy. This is because in most of the compounds, the Jahn-Teller axes cancel each other out due to the molecular symmetry. For example, it has been observed that a $\mathrm{Mn}_{10}$ "supertetrahedron", which is composed of a $\mathrm{Mn}_{4}{ }_{4} \mathrm{Mn}^{\mathrm{III}}{ }_{6}$ core and possesses a ground state spin of $S_{\mathrm{T}}=22$, has no significant anisotropy. ${ }^{86}$ More recently, a $\mathrm{Mn}_{19}$ cluster complex, which derives from two such tetrahedral units sharing a vertex, and has a record ground spin state of $83 / 2$, also not shows any significant anisotropy. ${ }^{87}$

Another strategy is to synthesize mixed transition metal/lanthanide (Ln) compounds, particularly Mn-Ln ones, as an attractive area. The strategy is obviously to take advantage of the lanthanide ion's significant spin, and/or its large anisotropy, as reflected in a large $D$ value, to generate SMMs distinctly different from the homometallic ones. Indeed, there are now several Mn-Ln SMMs, ${ }^{88,89,90}$ including $\mathrm{Mn}_{11} \mathrm{Ln}_{4},{ }^{88 \mathrm{a}} \mathrm{Mn}_{11} \mathrm{Gd}_{2},{ }^{89 \mathrm{a}} \mathrm{Mn}_{12} \mathrm{Gd}_{2},{ }^{88 \mathrm{c}} \mathrm{Mn}_{5} \mathrm{Dy}_{4},{ }^{89}$ 
${ }^{\mathrm{b}} \mathrm{Mn}_{2} \mathrm{Dy}_{2},{ }^{88 \mathrm{~b}}$ and $\mathrm{Mn}_{6} \mathrm{Dy}_{6}{ }^{90}$ Among them, $\mathrm{Mn}_{5} \mathrm{Dy}_{4}{ }^{89 \mathrm{~b}}$ has the highest energy barrier for $3 d-4 f$ SMMs at $38.6 \mathrm{~K}$, Although this value is less than those of pure $\mathrm{Mn}^{\text {III }}{ }^{67}$ or $\mathrm{Ln}^{\text {III }}$-based SMMs ${ }^{91}$ it indicates a promising avenue toward producing SMMs with higher energy barriers, with the challenge being to discover how the interplay of the mixed $3 d$ and $4 f$ ions can be optimized to achieve this goal. At present, many parameters need to be explored and a useful way forward is to compare properties for an isostructural series of compounds, as we show here.

\subsection{Syntheses, Structures and Magnetic Properties of Mixed Valent Manganese Clusters}

To synthesize high nuclearity metal clusters various kinds of ligands have been used till to date but it has been observed that alkoxy ligands are very efficient in this purpose because they can make different kinds of bridges between the metal centers. Special attention was paid in selecting the ligands to ensure that the ligands are flexible, multi-dentate and capable of forming $\mu$-oxo bridges between different metal centers. It was observed that tridentate diol ligands were appropriate candidates for the synthesis of polynuclear manganese clusters.

\subsubsection{Synthesis, Structure and Magnetic Behavior of Mixed Valent $\left[\mathrm{Mn}_{2}{ }_{2}\right.$ $\left.\mathrm{Mn}^{\mathrm{II}}\left(\mathrm{L}^{9}\right)_{2}(\mathrm{OAc})_{2}(\mathrm{OMe})_{2}(\mathrm{MeOH})_{2}\right] \cdot \mathrm{MeOH}(16)$}

Trinuclear mixed valent Mn complex 16 has been synthesized by reaction of 2-[3-(2Hydroxyphenyl)-1H-pyrazol-1-yl]ethanol $\left(\mathbf{H}_{2} \mathbf{L}^{\mathbf{9}}\right)$ with $\mathrm{Mn}(\mathrm{OAc})_{2} \cdot 4 \mathrm{H}_{2} \mathrm{O}$, in presence of ${ }^{\mathrm{n}} \mathrm{Bu}_{4} \mathrm{NOH}$ in 1:1.5:2 molar ratio in methanol at room temperature. The molecular structure is depicted in Figure 7.1. It includes a central octahedral $\mathrm{Mn}$ (II) ion, Mn2, flanked by two tetragonally distorted $\mathrm{Mn}(\mathrm{III})$ ions, $\mathrm{Mn} 1$, and $\mathrm{Mn} 3$. The separation between $\mathrm{Mn} 1 \cdots \mathrm{Mn} 2$ and $\mathrm{Mn} 2 \cdots \mathrm{Mn} 3$ are $3.062(4), 3.033(4) \AA$ respectively, and the angles between the Mn1 $\cdots$ Mn2 $\cdots$ Mn3 168.297(11) giving rise to almost linear structure. The coordination environment of the central $\mathrm{Mn} 2$ is composed of two oxygen atoms (O5, O8) from bridging ligand acetates, two alkoxy oxygen atoms $(\mathrm{O} 2, \mathrm{O} 11)$ from the ligand $\left[\left(\mathrm{L}^{9}\right)^{2-}\right]$, and two alkoxy oxygen atoms $(\mathrm{O} 3, \mathrm{O} 7)$ from two methoxy groups, forming a distorted octahedral structure. The Mn2-O bond lengths are in the range of 2.13-2.21 $\AA$ which are consistent with $\mathrm{Mn}(\mathrm{II})-\mathrm{O}$ distances reported in the literature. ${ }^{92}$ The coordination environment of Mn3 displays a distorted octahedral structure. The equatorial position of $\mathrm{Mn} 1$ is coordinated by the tridentate ligand $(\mathrm{O} 1, \mathrm{~N} 1, \mathrm{O} 2)$, an alkoxy oxygen atom $(\mathrm{O} 3)$ from the methoxy group, and axial position is occupied by one acetate oxygen $(\mathrm{O} 4)$, and one methanol molecule $(\mathrm{O} 4)$. The coordination environment of Mn3 also exhibits a distorted octahedral structure that is very similar to Mn1. 
The average bond length of Mn1 and Mn3 are in the range of 1.86-2.0 $\AA$ in equatorial, and 2.21-2.37 $\AA$ in axial which are typical for Mn(III) ion showing a Jahn-Teller elongation, where the Jahn-Teller axes of the two Mn(III) centres are almost parallel to each other. The methanol molecules are present as lattice solvent and form hydrogen bonds with the

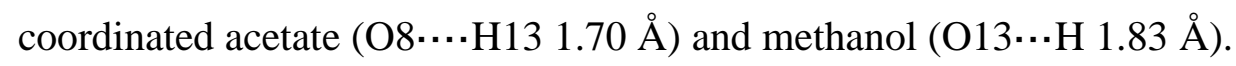

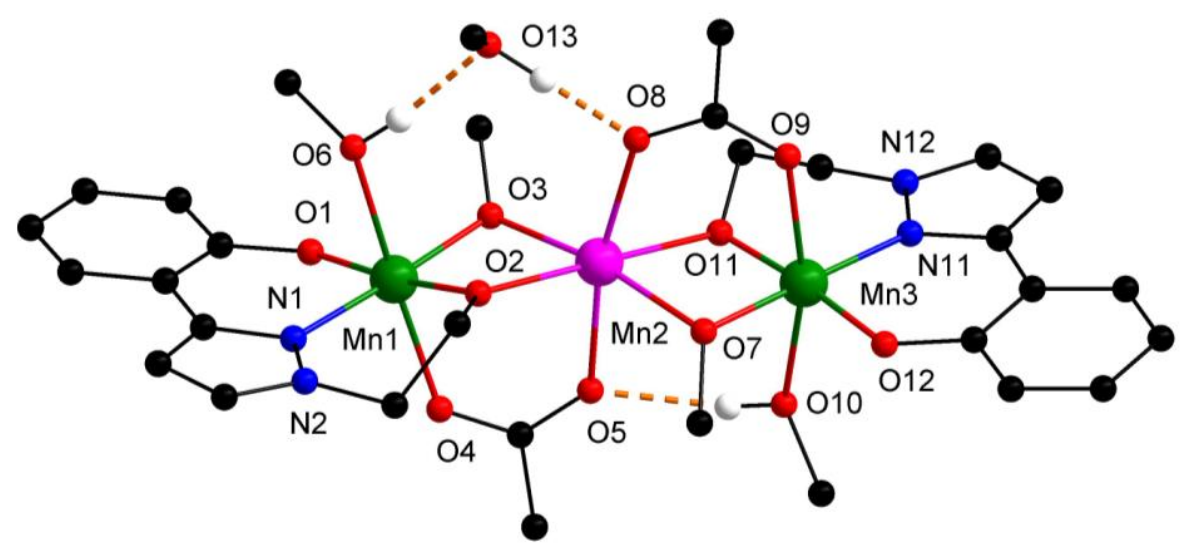

Figure 7.1. Molecular structure of trinuclear cluster 16. Colour code $\mathrm{Mn}(\mathrm{III})$ green, $\mathrm{Mn}$ (II) purple, $\mathrm{N}$ blue, $\mathrm{O}$ red, $\mathrm{C}$ black, $\mathrm{H}$ white.

The magnetic susceptibility of $\mathbf{1 6}$ was measured from $300 \mathrm{~K}$ to $2 \mathrm{~K}$ in order to characterize the exchange coupling within the mixed valent trinuclear core of Mn. No significant field dependence was observed when data were measured at applied fields of 0.2 and $0.5 \mathrm{~T}$. The temperature dependence of the molar magnetic susceptibility $\chi_{\mathrm{M}}$ and of the product $\chi_{\mathrm{M}} T$ is shown in Figure 7.2. The observed $\chi_{\mathrm{M}} T$ value at $295 \mathrm{~K}$ is $10.13 \mathrm{~cm}^{3} \mathrm{Kmol}^{-1}$ (corresponding to an effective moment $\left.\mu_{\mathrm{eff}}=9.0 \mu_{\mathrm{B}}\right)$, this value is agreement with the value $\left(10.36 \mathrm{~cm}^{3} \mathrm{Kmol}^{-1}\right.$ or $\left.\mu_{\mathrm{eff}}=9.11 \mu_{\mathrm{B}}\right)$ expected for the presence of one uncoupled $\mathrm{Mn}(\mathrm{II})(S=5 / 2, g=2.0)$ and two uncoupled $\mathrm{Mn}(\mathrm{III})$ ions $(S=2, g=2.0)$. Upon decreasing the temperature the $\chi_{\mathrm{M}} T$ product continuously decreases to reach $1.91 \mathrm{~cm}^{3} \mathrm{Kmol}^{-1}$ (corresponding to an effective moment $\mu_{\mathrm{eff}}=$ $\left.3.91 \mu_{\mathrm{B}}\right)$ at $2 \mathrm{~K}$, indicating a dominant antiferromagnetic interaction and non zero ground state spin for 16. 


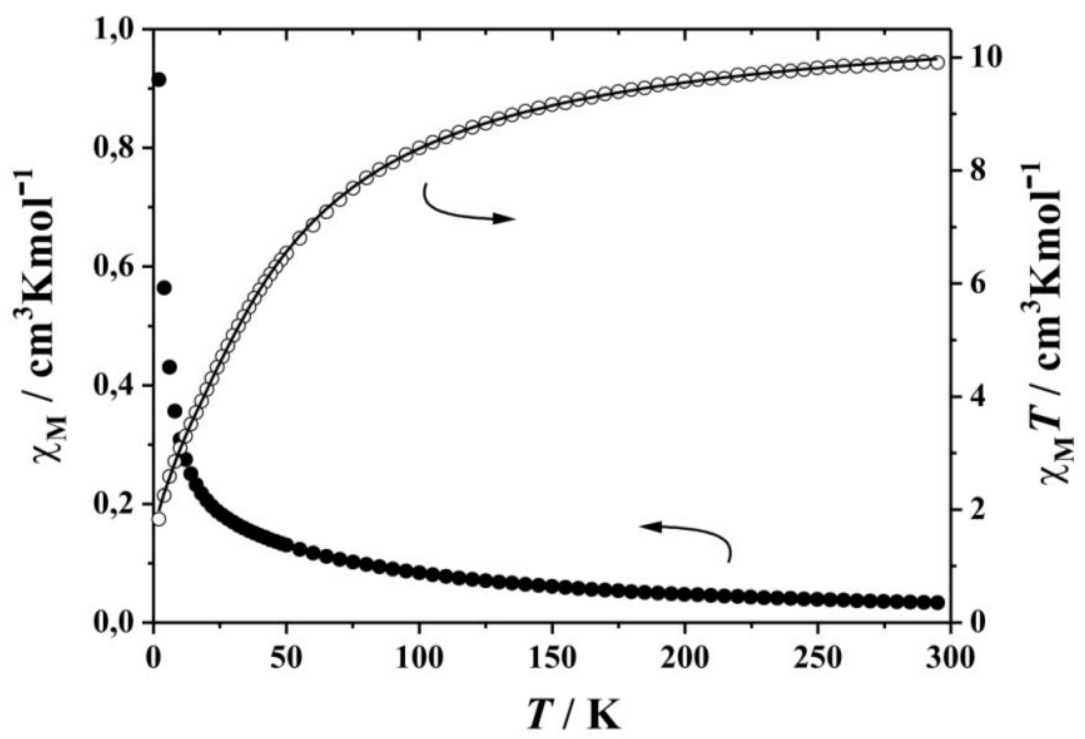

Figure 7.2. Plots of $\chi_{\mathrm{M}}$ (solid circles) and $\chi_{\mathrm{M}} T$ (open circles) versus temperature for $\mathbf{1 6}$ at $0.5 \mathrm{~T}$. The solid lines represent the simulated curves.

The fitting of the magnetic data for this complex was done using the following Hamiltonian:

$$
H=-2 J\left(S_{1} S_{2}+S_{2} S_{3}\right)
$$

Where, $J$ is the exchange interaction in the trimer between adjacent $\mathrm{Mn}(\mathrm{II})$ and $\mathrm{Mn}$ (III) ions; $S_{1}=S_{3}=2$ for $\mathrm{Mn}(\mathrm{III})$ and $S_{2}=5 / 2$ for Mn(II) ions. Fitting the data gave $J=-2.63 \mathrm{~cm}^{-1}$, $g_{\mathrm{Mn}(\mathrm{III})}=2.16, g_{\mathrm{Mn}(\mathrm{II})}=1.87,|D|=5.9 \mathrm{~cm}^{-1}$.

To determine the spin ground state, magnetization data were collected at 1,3 and $5 \mathrm{~T}$ in the temperature range 2-290 K (Figure 7.3) and plotted as reduced magnetization $(M / N g \beta)$ vs. $\left(\mu_{\mathrm{B}} B / k T\right)$, where $N$ is the Avogadro's number, $\beta$ is the Bohr magneton and $k$ is the Boltzmann's constant. For a system occupying only the ground state and experiencing no zero-field splitting $(D)$, the various isofield lines would be superimposed and $M / N \beta$ would saturate at a value $S$. The non-superposition of the variable temperature variable field (VTVH) plots at low temperature clearly indicates the presence of zero-field splitting (ZFS or $D$ ), and the reduced magnetization measurement yielded a ground state $S_{\mathrm{T}}=3 / 2$. The $M$ versus $H$ measurements at $2 \mathrm{~K}$ (Figure 7.3, bottom) reveals that the magnetisation reaches a value of $3.43 N \beta$ at $5 \mathrm{~T}$ which is close to the expected $S=3 / 2$ value of $3 N \beta$ for the $\mathrm{Mn}_{2}^{\mathrm{III}}{ }_{2} \mathrm{Mn}^{\mathrm{II}}$ system, indicating the presence of antiferromagnetic interaction between the manganese(III) and $\mathrm{Mn}$ (II) ions. However there is no any true saturation occur upto $5 \mathrm{~T}$, indicating presence of magnetic anisotropy and or low lying excited states. Attempts to fit the data by using the 
method of full-matrix diagonalization of the spin Hamiltonian matrix including axial ZFS produced best fits with, $J=-2.90 \mathrm{~cm}^{-1}, g_{\mathrm{Mn}(\mathrm{III})}=2.2, g_{\mathrm{Mn}(\mathrm{II})}=2.0,|D|=3.0 \mathrm{~cm}^{-1}$. Those "J" and $g$ values are close to the values evaluated from the susceptibility measurements at $1 \mathrm{~T}$ described earlier and thus confirm the credibility of the simulated parameters. The variable temperature variable field (VTVH) plot is shown in the Figure 7.3 (top). It has alraedy been outlined that the main source of the molecular anisotropy is due to presence of Jahn-Teller distorted $\mathrm{Mn}^{\mathrm{III}}$ ions. The projections of this single-ion anisotropy onto the molecular anisotropy axis will determine the molecular parameter $D$.
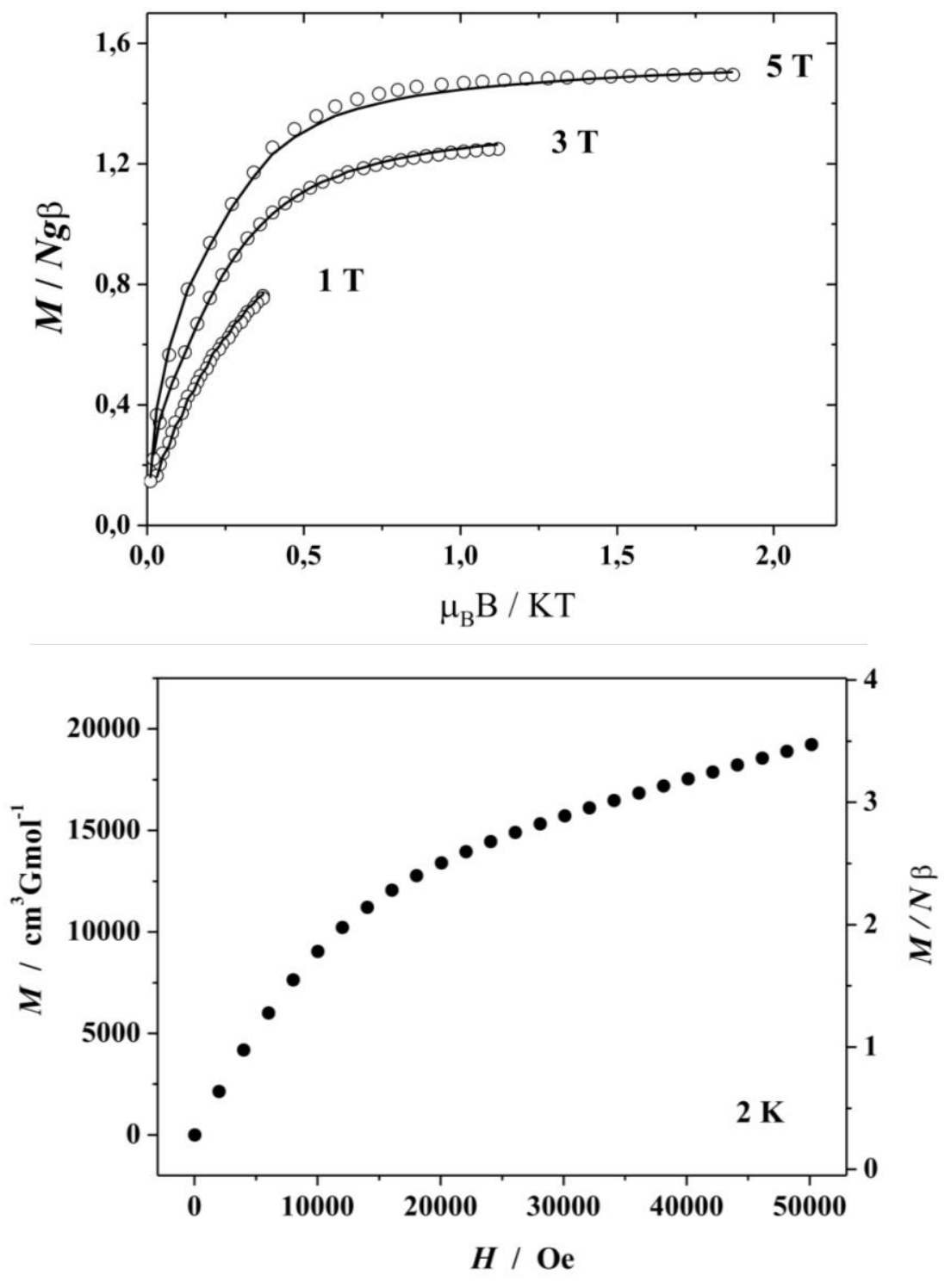

Figure 7.3. Plot of reduced magnetization $\left(M / N g \beta\right.$ ) vs. $\left(\mu_{\mathrm{B}} B / k T\right)$ (top), and magnetisation $M$ (or $M / N \beta$ ) versus applied field $H$ (bottom) for $\mathbf{1 6}$ at $2 \mathrm{~K}$. 
As we already mentioned earlier that the Jahn-Teller axis of two manganese (III) ions are almost parallel to each other, so it is expected compound $\mathbf{1 6}$ possesses significant magnetic anisotropy $(D)$ and it may reflect the slow relaxation of magnetisation across the energy barrier in single molecule magnet (SMM). To get more insight into the characterisation of the system precise $S_{\mathrm{T}}$ and $D$ values are required. In order to determine these values and fully elucidate the sign of the ZFS $(D)$, high-frequency EPR (HFEPR) technique is needed that under progress.
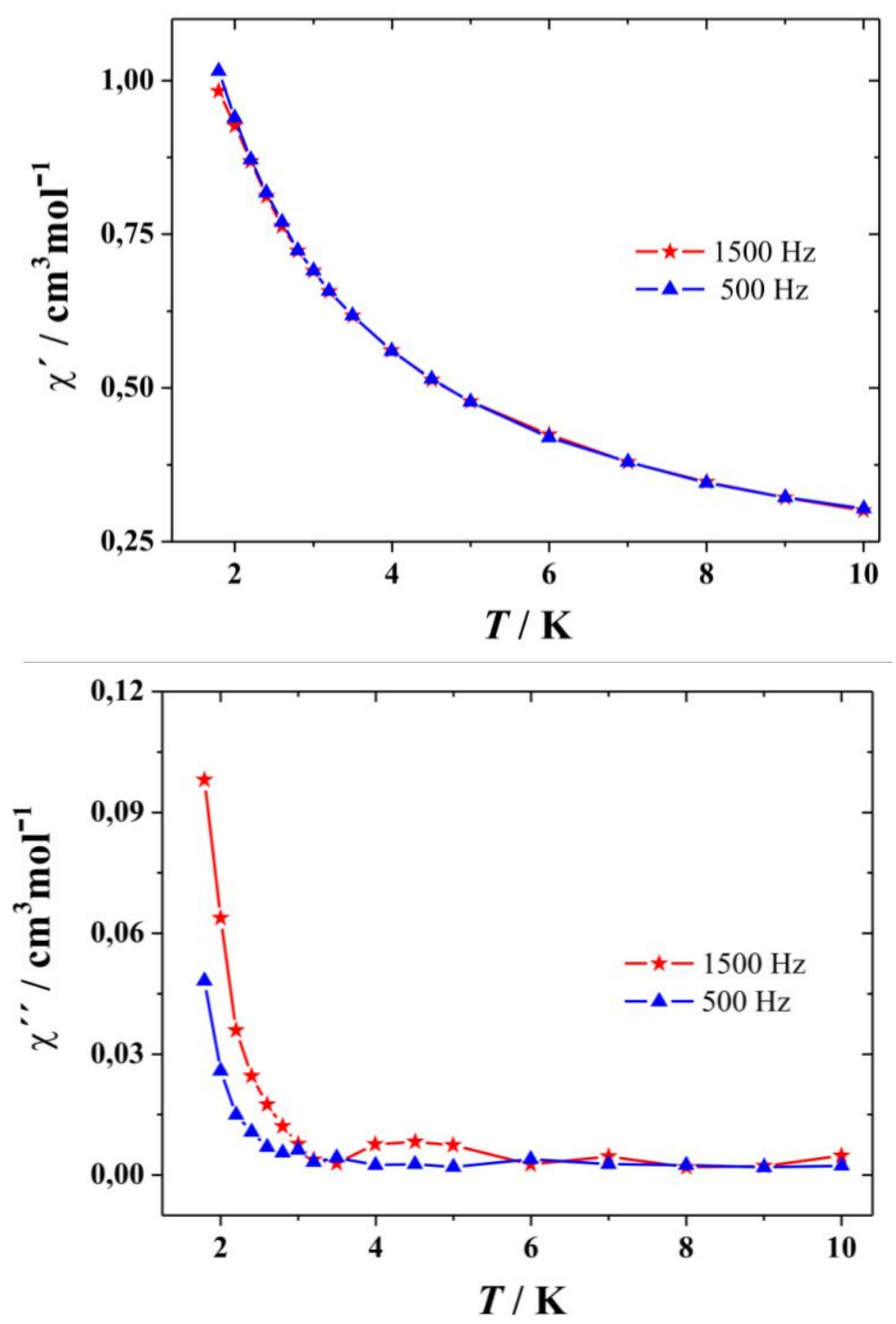

Figure 7.4. Plots of in-phase ( $\chi^{\prime}$, top) and out-of-phase $\left(\chi^{\prime}{ }^{\prime}\right.$, bottom) ac susceptibilities versus temperature for complex 16.

To investigate whether $\mathbf{1 6}$ might be a SMM, ac susceptibility measurements were performed in the two frequencies 500, and $1500 \mathrm{~Hz}$ at temperatures between 2 and $10 \mathrm{~K}$ and with a zero applied dc field (Figure 7.4). 
Below circa $3 \mathrm{~K}$, a frequency-dependent decrease in the in-phase ( $\left.\chi^{\prime}\right)$ signal (Figure 7.4; top) and a concomitant increase in the out-of-phase $\left(\chi^{\prime \prime}\right)$ signal (Figure 7.4; bottom) were observed. Such $\chi^{\prime \prime}$ signals suggest that the complex might be a SMM. To confirm SMM behavior, the measurements of magnetization vs. direct current field of single crystals of $\mathbf{1 6}$ using a micro-hall apparatus at low temperature is in progress.

\subsubsection{Synthesis, Structure and Magnetic Behavior of Hexanuclear $\left[\mathrm{Mn}_{4}^{\mathrm{III}}\right.$ $\left.\mathrm{Mn}_{2}{ }_{2}\left(\mathrm{~L}^{9}\right)_{4} \mathrm{Cl}_{4}\left(\mathrm{H}_{2} \mathrm{O}\right)_{4}\left(\mu_{4}-\mathrm{O}\right)_{2}\right] \cdot 4 \mathrm{CH}_{3} \mathrm{CN}(17)$}

The reaction of $\mathbf{H}_{2} \mathbf{L}^{9}$ with $\mathrm{MnCl}_{2} \cdot 4 \mathrm{H}_{2} \mathrm{O}$ and $\mathrm{Mn}\left(\mathrm{ClO}_{4}\right)_{2} \cdot 6 \mathrm{H}_{2} \mathrm{O}$ in presence of $\mathrm{NEt}_{3}$ in dry acetonitrile under inert atmosphere at room temperature gave a block shaped red crystal of $\mathbf{1 7}$. X-ray crystallography showed that compound $\mathbf{1 7}$ crystallizes in the monoclinic space group $P 2_{1} / n$. The molecular structure of compound $\mathbf{1 7}$ is showed in Figure 7.5, and selected bond lengths, bond angles, and structural core are shows in Figure 7.6. The central core of compound 17 contains $\left[\mathrm{Mn}_{4}^{\mathrm{III}} \mathrm{Mn}_{2}{ }_{2}\left(\mu_{4}-\mathrm{O}\right)_{2}(\mu-\mathrm{Cl})_{2}\right]^{10+}$ ladder like unit possess incomplete face-sharing triple cubane core. The asymmetric unit contains a half of the entire molecule, and a crystallographic inversion center is located at the center of the core. The cluster core consists of six manganese ions, two phenoxide $\mathrm{O}$ atoms, two $\mu_{4}-\mathrm{O}$ atoms, and four alkoxide $\mathrm{O}$ atoms from $\left(\mathbf{L}^{\mathbf{9}}\right)^{2-}$, and two chloride atoms (Figure 7.6). The oxidaion state of the metal ions is confirmed by bond valence sum calculations ${ }^{74}$ and the relatively short bonds are assigned as the $\mathrm{Mn}^{\mathrm{III}}$ ions (see Figure 7.6). The structure consists of two $\mathrm{Mn}^{\mathrm{II}}$ and four $\mathrm{Mn}^{\mathrm{III}}$, and those are bridged by alkoxide $\mathrm{O}$ atoms, and two $\mathrm{Cl}$ atoms forming a distorted incomplete cube structure. The average bond distance of $\mathrm{Mn}(1,3)-\mathrm{O}(1,2,3,4,5)(1.87-2.00 \AA)$ are noticeably shorter compare to $\mathrm{Mn}(2)-\mathrm{O}\left(2,2^{\prime}, 5,5^{\prime}\right)(2.14-2.20 \AA)$ in equatorial. According to the bond length data, $\mathrm{Mn}^{\mathrm{II}}$ are assigned as central $\mathrm{Mn}(2)$ and $\mathrm{Mn}\left(2^{\prime}\right)$, and $\mathrm{Mn}^{\mathrm{III}}$ ions are attributed as $\mathrm{Mn} 1, \mathrm{Mn}^{\prime}$, Mn3, and Mn3 ${ }^{-92}$ The coordination enviornment of all Mn ions exhibit distorted octahedral geometry. The equatorial position of $\mathrm{Mn} 1$ is occupied by $(\mathrm{O} 1, \mathrm{~N} 1, \mathrm{O} 2)$ atoms from the ligand $\left(\mathrm{L}^{9}\right)^{2-}$, and a $\mu_{4}$-oxo $(\mathrm{O} 5)$ atom, and axial positions are occupied by a phenoxo oxygen $(\mathrm{O} 3)$ atom from another ligand, and a water molecule (O6). The coordination environment of Mn3 is also similar to Mn1, where equatorial position is capped by tridentate ligand (O3, N3, O4) and a $\mu_{4}$-oxo (O5) atom, and axial position is occupied by a $\mu$-chloro (Cl1), and a water molecule (O7). The coordination surrounding of Mn2 also displays a distorted octahedral geometry, where axial positions are filled by $\mu$-chloro $(\mathrm{Cl} 2)$, and a $\mu_{4}$-oxo (O5) atom, and equatorial position is engaged by $\left(\mathrm{O} 2, \mathrm{O}^{\prime}, \mathrm{O}^{\prime}, \mathrm{Cl1}\right)$. 


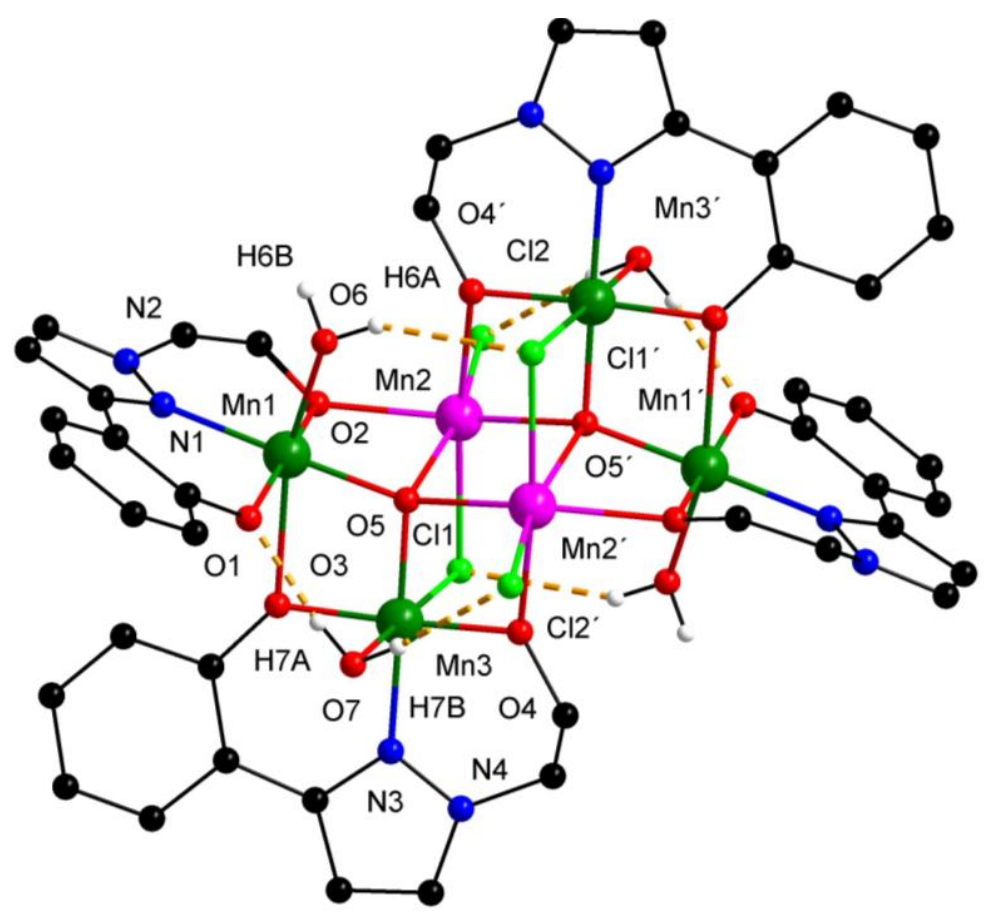

Figure.7.5. View of the molecular structure of complex 17. In the interests of clarity all the hydrogen atoms of the ligand have been omitted. Colour code $\mathrm{Mn}(\mathrm{III})$ green, $\mathrm{Mn}(\mathrm{II})$ purple, $\mathrm{N}$ blue, $\mathrm{Cl}$ green, $\mathrm{O}$ red, $\mathrm{C}$ black, $\mathrm{H}$ white.

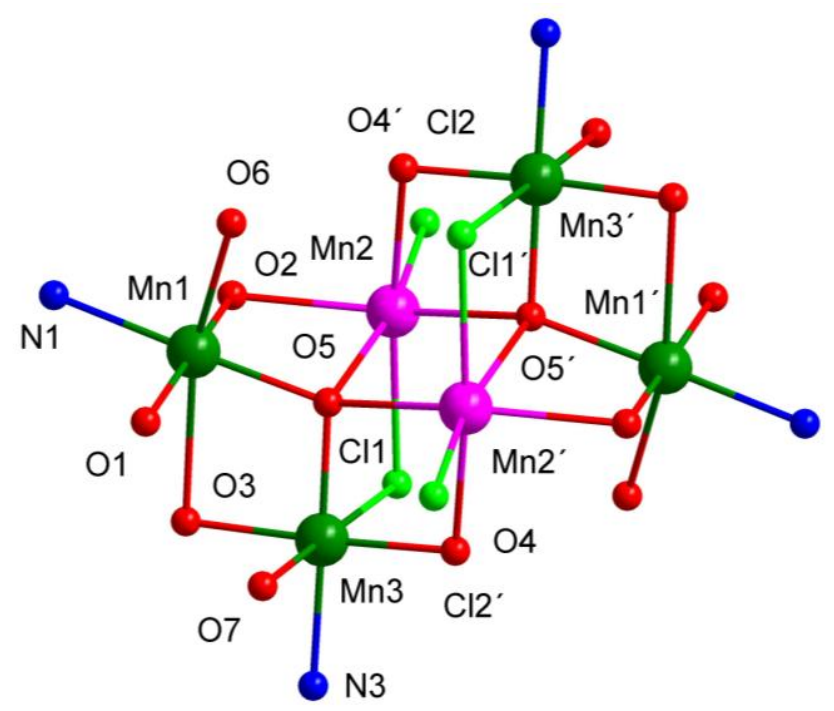

Figure.7.6. View of the molecular structure of metal-X $(\mathrm{X}=\mathrm{O}, \mathrm{Cl})$ core of complex 17. Selected interatomic distances [̊] and angles [] ]: Mn1-O1 1.896(2), Mn1-O2 1.886(31), Mn1-O5 1.91(32), Mn1-N1 1.886(31), Mn1N1 2.004(35), Mn1-O3 2.450(27), Mn1-O6 2.21(38), Mn2-O2 2.141(31), Mn2-O5' 2.184(32), Mn2-O4' 2.20(27), Mn2-Cl1 2.521(12), Mn2-Cl2 2.484(13), Mn2-O5 2.358(30), Mn2-Cl2 2.484(13), Mn3-O3 1.900(32), Mn3-O4 1.870(32), Mn3-N3 1.996(38), Mn3-O5 1.893(27), Mn3-O7 2.29(32), Mn3-Cl1 2.669(12), H6A-Cl1 2.5214(6215), O1-H7A 1.8727(594), H7B-Cl2 2.3158(522), Mn1-O2-Mn2 105.099(129), Mn1-O5-Mn2 96.665(108), Mn1-O5-Mn3 106.516(143), Mn1-O3-Mn3 87.914(111), Mn2-O5-Mn3 107.155(124), Mn2-Cl1Mn3 82.740(36). Symtmetry transformation used to generate equivalent atoms: ( $\left.{ }^{\prime}\right) 1-\mathrm{x}, 1-\mathrm{y}, 1-\mathrm{z}$. 
The average bond length of Mn1-O or (Mn3-O) are 1.88-2.00 $\AA$ or (1.87-1.99 $\AA$ ) in equatorial, and 2.20-2.45 $\AA$ or (2.28-2.66 $⿱$ ) in axial which are typical for $\mathrm{Mn}^{\mathrm{III}}$ ion showing Jahn-Teller elongation. The Jahn-Teller axes of the $\mathrm{Mn}^{\mathrm{III}}$ ions in the core are orthogonal to each other. In addition there is intra-, and intermolecular hydrogen bonding contact found within the crystal lattice. The coordinated water molecules is intermolecularly hydrogen

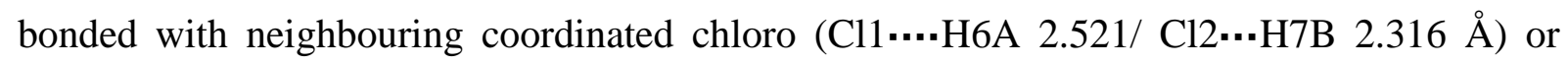
phenoxo oxygen atom $(\mathrm{O} 1 \cdots \mathrm{H} 7 \mathrm{~A} 1.872 \AA)$, and acetontrile as a lattice solvent which involes in intermolecular hydrogen bonding with $\mathrm{Cl}$ atom of the cluster, formed a 3-D networkers, that is not shown here for simplicity.

The magnetic susceptibility of the compound 17 was measured under $0.1 \mathrm{~T}$ field in the 2-300 $\mathrm{K}$ temperature range in order to characterize the exchange coupling within the core of $\left[\mathrm{Mn}_{4}^{\mathrm{III}} \mathrm{Mn}_{2}{ }_{2}\left(\mu_{4}-\mathrm{O}\right)_{2}(\mu-\mathrm{Cl})_{2}\right]^{10+}$.

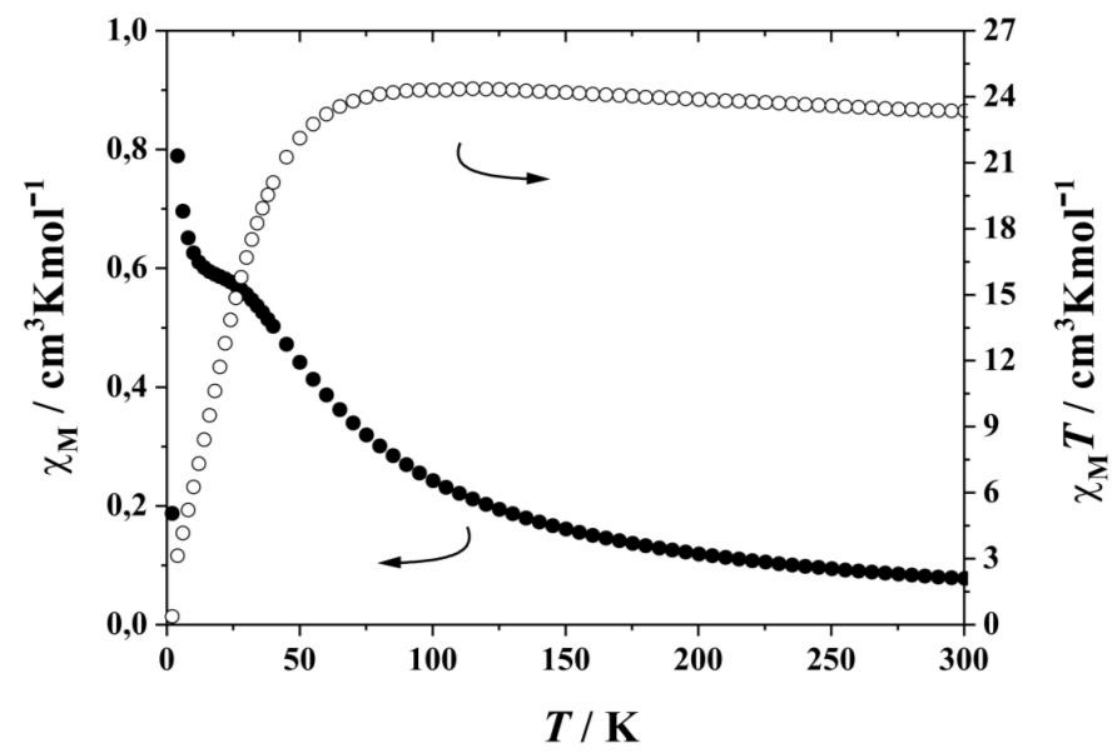

Figure 7.7. Plots of $\chi_{\mathrm{M}}$ (solid circles) and $\chi_{\mathrm{M}} T$ (open circles) versus temperature for $\mathbf{1 7}$ at $0.1 \mathrm{~T}$.

The temperature dependence of the molar magnetic susceptibility $\chi_{\mathrm{M}}$ and of the product $\chi_{\mathrm{M}} T$ is shown in Figure 7.7. The $\chi_{\mathrm{M}} T$ value per cluster at $300 \mathrm{~K}$ is $23.3 \mathrm{~cm}^{3} \mathrm{Kmol}^{-1}$ (corresponding to an effective moment $\mu_{\text {eff }}=13.66 \mu_{\mathrm{B}}$ ), slightly higher than the theoretical value $(20.7$ $\mathrm{cm}^{3} \mathrm{Kmol}^{-1}$ or $\mu_{\mathrm{eff}}=12.9 \mu_{\mathrm{B}}$ for $\left.g=2\right)$ expected for four uncoupled $\mathrm{Mn}$ (III) $(S=2, g=2.0)$, and two uncoupled $\mathrm{Mn}(\mathrm{II})$ ions $(S=5 / 2, g=2.06)$. Upon lowering the temperature, $\chi_{\mathrm{M}}$ goes through a broad maximum at $27 \mathrm{~K}$ and $\chi_{\mathrm{M}} T$ gradually tends to zero, in accordance with overall intramolecular antiferromagnetic coupling and an $S_{\mathrm{T}}=0$ ground state. The individual exchange interaction between the $\mathrm{Mn} 1 \cdots \mathrm{Mn} 2, \mathrm{Mn} 1 \cdots \mathrm{Mn} 3$, and Mn2 $\cdots \mathrm{Mn} 3$ pairs, are underway. 


\subsection{Synthesis, Structure and Magnetic behavior of Heteronuclear [NHEt ${ }_{3}$ ]}

$\left[\left(\mathrm{L}^{9}\right)_{2} \mathrm{Mn}^{\mathrm{III}}{ }_{2}\left\{\left(\mathrm{NO}_{3}\right)_{2} \mathrm{Ln}^{\mathrm{III}}\right\}_{2}\left(\mathrm{O}_{2} \mathrm{CPh}\right)_{3}\left(\mu_{4}-\mathrm{O}\right)\right] \cdot \mathbf{x} \mathrm{CH}_{3} \mathrm{CN} \cdot \mathrm{y} \mathrm{CH}_{2} \mathrm{Cl}_{2}\left[\mathrm{Ln}=\mathrm{Gd}^{\mathrm{III}}\right.$ (18), Tb $^{\text {III }}$ (19), Dy ${ }^{\text {III }}$ (20)]

In the previous chapter we already shown that the pyrazole based tridentate diol ligand $\mathbf{H}_{2} \mathbf{L}^{9}$ is very efficient in preparing the heterobimetallic compounds. The interesting aspect of $\mathbf{H}_{2} \mathbf{L}^{9}$ is that it possesses three coordination sites. Two of those sites are hard donor phenoxy and alkoxy tend to bind to oxophilic lanthanide ions, and the remaining one is the soft-donor nitrogen tends to bind to transition metal ions. Thus sequential reaction of deprotonated ligand of $\mathbf{H}_{2} \mathrm{~L}^{9}$ with $\mathrm{Mn}\left(\mathrm{O}_{2} \mathrm{CPh}\right)_{2} \cdot 2 \mathrm{H}_{2} \mathrm{O}$ followed by reaction with the appropriate $\mathrm{Ln}\left(\mathrm{NO}_{3}\right)_{2} \cdot 6 \mathrm{H}_{2} \mathrm{O}$ $\left[\mathrm{Ln}=\mathrm{Gd}^{\mathrm{III}}(\mathbf{1 8}), \mathrm{Tb}^{\mathrm{III}}(\mathbf{1 9}), \mathrm{Dy}^{\mathrm{III}}(\mathbf{2 0})\right]$ afforded heterobimtallic compounds 18-20 in $39-52 \%$ yield.

Complexes 18-20 are isostructural and crystallize in a monoclinic unit cell (space group: $P$ $2{ }_{1} / c$ ). Here molecular structure of compound $\mathbf{1 8}$ is depicted in Figure 7.8 as an example. The structural core is shown in Figure 7.9. Relevant atom distances and bond angles are collected in Table 7.1

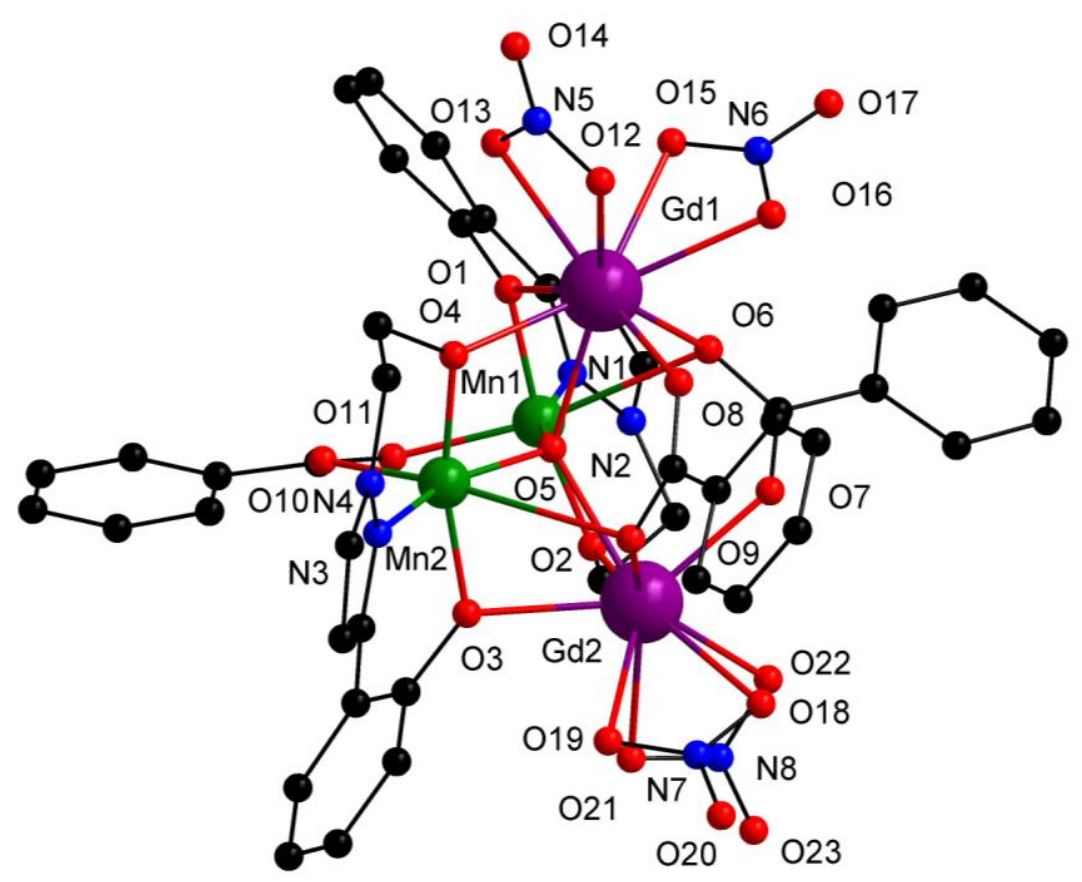

Figure 7.8. View of the molecular structure of compound 18. Organic $\mathrm{H}$ atoms, and counter cation $\left[\mathrm{NHEt}_{3}\right]^{+}$are omitted for clarity. Colour code O-red, N-blue, C-black, $\mathrm{Mn}^{\mathrm{III}}$ green, $\mathrm{Gd}^{\mathrm{III}}$ firebrick. 


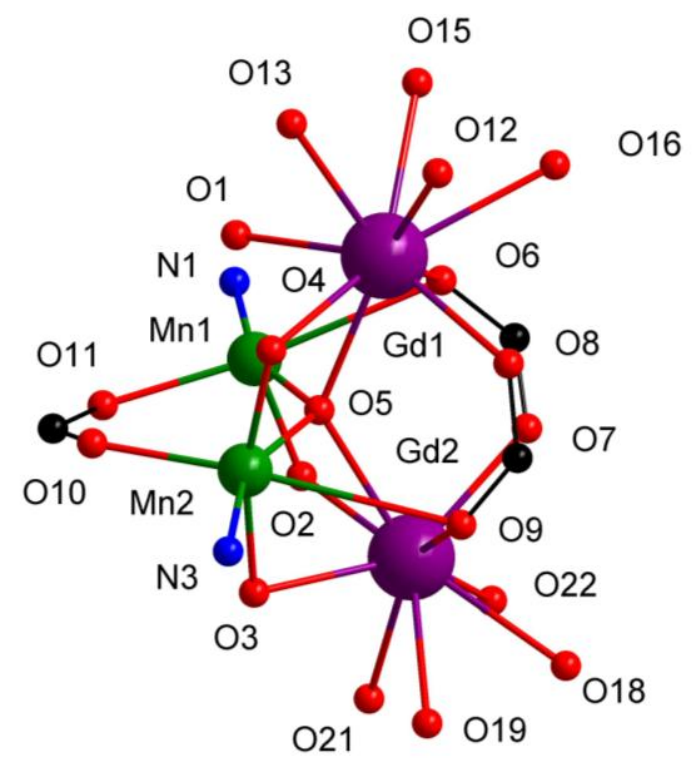

Figure 7.9. View of the molecular structure of $\left[\mathrm{Mn}_{2} \mathrm{Gd}_{2}\left(\mu_{4}-\mathrm{O}\right)\right]^{10+}$ core of compound 18. Colour code O-red, Nblue, C-black, $\mathrm{Mn}^{\mathrm{III}}$ green, $\mathrm{Gd}^{\mathrm{III}}$ firebrick.

Table 7.1. Selected interatomic distances $[\AA]$ and angles $\left[{ }^{\circ}\right]$ of compound $\mathbf{1 8}$

\begin{tabular}{|c|c|}
\hline \multicolumn{2}{|c|}{18} \\
\hline Mn1-O1 1.9096(30) & Mn2-O3 1.8974(33) \\
\hline Mn1-N1 1.9880(36) & Mn2-N3 1.9976(36) \\
\hline Mn1-O5 1.8612(30) & Mn2-O5 1.8651(28) \\
\hline Mn1-O11 2.0818(33) & Mn2-O10 2.0719(33) \\
\hline Mn1-O2 1.8735(30) & Mn2-O4 1.8740(35) \\
\hline Mn1-O6 2.6419(37) & Mn1 $\cdots$ Mn2 3.2546(10) \\
\hline Gd1-O1 2.428(30) & Gd2-O3 2.4168(29) \\
\hline Gd1-O4 2.3997(31) & Gd2-O2 2.3729(31) \\
\hline Gd1-O5 2.3738(33) & Gd2-O5 2.4177(27) \\
\hline Gd1-O6 2.4177(31) & Gd1-O7 2.3200(37) \\
\hline Mn1-O5-Mn2 121.716(153) & Mn1-O5-Gd1 99.600(125) \\
\hline Mn1-O1-Gd1 99.366(199) & Mn2-O5-Gd2 99.886(124) \\
\hline Mn1-O2-Gd2 104.600(137) & Gd1-O5-Gd2 129.467(128) \\
\hline Mn1-O6-Gd1 79.846(98) & Mn2-O3-Gd2 98.977(131) \\
\hline Mn2-O4-Gd1 103.880(138) & Gd1‥G Gd2 4.3331(3) \\
\hline
\end{tabular}

The central core of compound 18 contain $\left[\mathrm{Mn}_{2} \mathrm{Gd}_{2}\left(\mu_{4}-\mathrm{O}\right)\right]^{10+}$ like unit with the two $\mathrm{Mn}^{\mathrm{III}}$ and two $\mathrm{Gd}^{\mathrm{III}}$ arranged in a distorted tetrahedral shaped by a $\mu_{4}-\mathrm{O}$ bridge. Both $\mathrm{Mn}$ atoms are coordinated by $\{\mathrm{ONO}\}$ chelate arms of the ligand $\left(\mathbf{H}_{2} \mathbf{L}^{\mathbf{9}}\right)$, and rest position is occupied by an $\mu_{4}$-oxo atom, and syn, syn-benzoate bridging ligand formed a six coordinated distorted octahedral structure. The interatomic distance between the Mn $\cdots \mathrm{Mn}$ is $3.26 \AA$. Gd and Mn atoms are bridged by an alkoxide $\mathrm{O}$ atom, a phenoxide $\mathrm{O}$ atom from the ligand, one syn, antibenzoate bridge, and the $\mathrm{Mn}^{\mathrm{III}} \ldots \mathrm{Gd}^{\mathrm{III}}$ distances range from 3.2516(8) to 3.3805(8) $\AA$. Both $\mathrm{Gd}$ 
atoms are interconected by a $\mu_{4}$-oxo atom and two syn, syn-benzoate bridging ligand. The interatomic distance between the Gd $\cdots \mathrm{Gd}$ is $4.33 \AA$. The average bond distances of Mn1-O(1, 2, 5)(1.86-1.91 $\AA$ ) or $\operatorname{Mn} 2-\mathrm{O}(3,4,5)(1.86-1.90 \AA)$ in equatorial is noticeably shorter compared to $\mathrm{Mn} 1-\mathrm{O}(11,6)(2.08-2.64 \AA)$ or $\mathrm{Mn} 2-\mathrm{O}(9,10)(2.07-2.89 \AA)$ in axial position, suggest that both Mn atoms have significant Jahn-Teller elongation.

Magnetic susceptibility data was collected for all complexes in the temperature range from 300 to $2.0 \mathrm{~K}$ in order to characterize the exchange coupling within the $\left[\mathrm{Mn}_{2}{ }_{2}{ }^{\mathrm{III}}{ }^{\mathrm{III}}{ }_{2}\left(\mu_{4}-\mathrm{O}\right)\right]^{10+}$ core. No significant field dependence was observed when data was measured at applied fields of 0.5 , and $0.1 \mathrm{~T}$ for $\mathbf{1 8}, 0.5$, and $0.05 \mathrm{~T}$ for $\mathbf{1 9}$, and 0.5 , and $0.2 \mathrm{~T}$ for $\mathbf{2 0}$. The temperature dependence of the molar magnetic susceptibility $\chi_{\mathrm{M}}$ and of the product $\chi_{\mathrm{M}} T$ is shown in Figure 7.10.
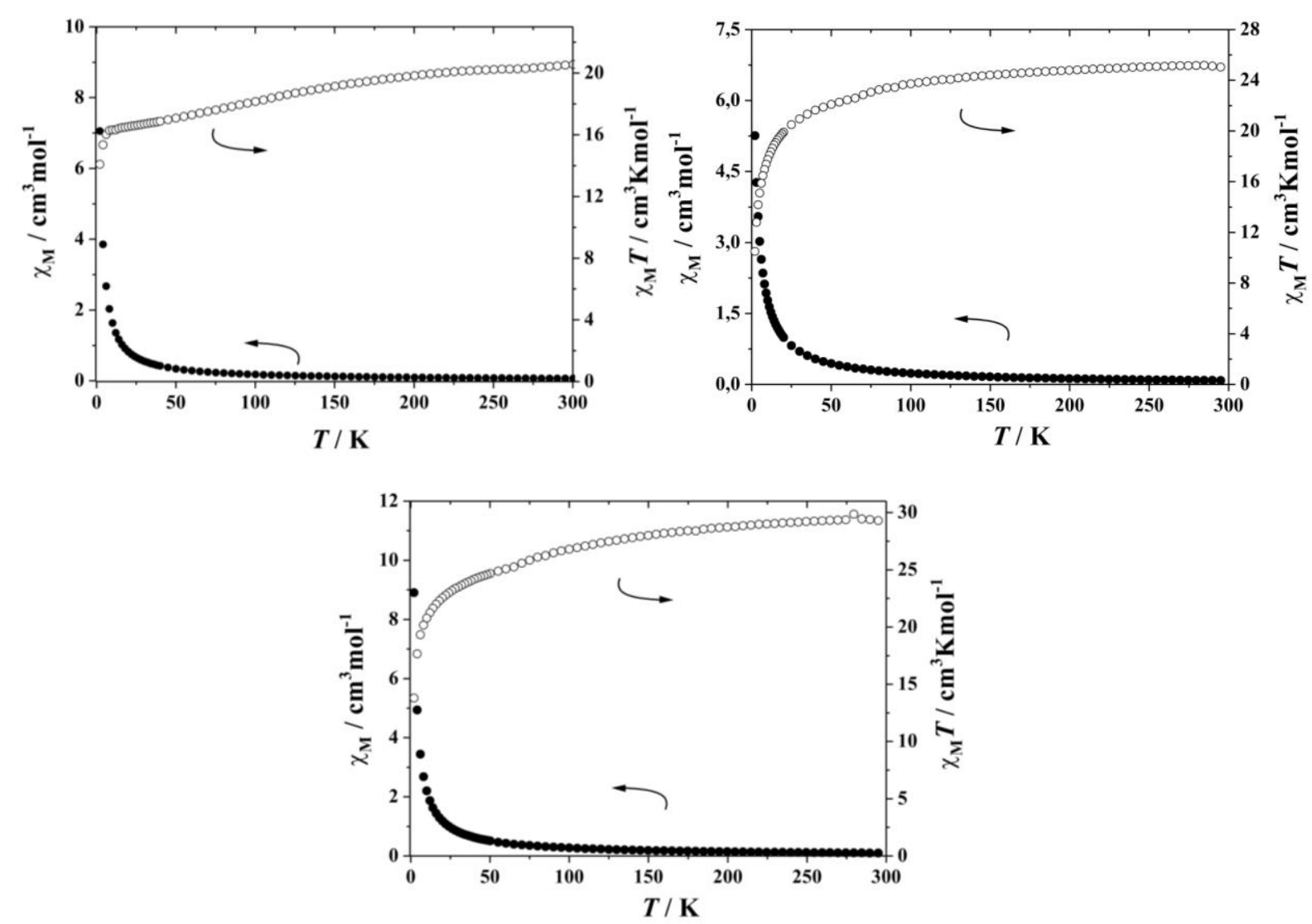

Figure 7.10. Plots of $\chi_{\mathrm{M}}$ (solid circles) and $\chi_{\mathrm{M}} T$ (open circles) versus temperature for 18 at $0.5 \mathrm{~T}$ (left-top), 19 at $0.5 \mathrm{~T}$ (right-top), and 20 at $0.5 \mathrm{~T}$ (bottom).

The observed $\chi_{\mathrm{M}} T$ value at $300 \mathrm{~K}$ is $20.6 \mathrm{~cm}^{3} \mathrm{Kmol}^{-1}$ (corresponding to an effective moment $\left.\mu_{\text {eff }}=12.84 \mu_{\mathrm{B}}\right)$, close to the value $\left(21.7 \mathrm{~cm}^{3} \mathrm{Kmol}^{-1}\right.$ or $\left.\mu_{\text {eff }}=13.19 \mu_{\mathrm{B}}\right)$ expected for the 
presence of two uncoupled $\mathrm{Mn}^{\mathrm{III}}(S=2, g=2.0)$ and two uncoupled $\mathrm{Gd}^{\mathrm{III}}$ ions $\left({ }^{8} S_{7 / 2}, S=7 / 2\right.$, $L=0$ ) for 18 . The $\chi_{\mathrm{M}} T$ value decreases gradually with decreasing temperature to reach 16.4 $\mathrm{cm}^{3} \mathrm{Kmol}^{-1}$ (corresponding to an effective moment $\mu_{\mathrm{eff}}=11.5 \mu_{\mathrm{B}}$ ) at $8 \mathrm{~K}$, consistent with dominant antiferromagnetic exchange interactions within complex 18. Below $8 \mathrm{~K}$, the $\chi_{\mathrm{M}} T$ value decreases steeply to reach $14.2 \mathrm{~cm}^{3} \mathrm{Kmol}^{-1}$ (corresponding to an effective moment $\mu_{\mathrm{eff}}=$ $\left.10.7 \mu_{\mathrm{B}}\right)$ at $2 \mathrm{~K}$ indicating a non zero ground state spin for $\mathbf{1 8}$.

For complexes 19 and 20, the $\chi_{\mathrm{M}} T$ values at all temperatures are higher than the corresponding ones for 18 (Figure 7.10). The observed $\chi_{\mathrm{M}} T$ value at $300 \mathrm{~K}$ is $25.3 \mathrm{~cm}^{3} \mathrm{Kmol}^{-}$ ${ }^{1}$, less than the value $\left(29.6 \mathrm{~cm}^{3} \mathrm{Kmol}^{-1}\right)$ expected for the presence of two uncoupled $\mathrm{Mn}^{\mathrm{III}}(S=$ 2, $g=2.0)$ and two uncoupled $\mathrm{Tb}^{\mathrm{III}}$ ions $\left({ }^{7} F_{6}, S=3, L=3\right)$ for 19. The $\chi_{\mathrm{M}} T$ value decreases gradually with decreasing temperature to reach $21.4 \mathrm{~cm}^{3} \mathrm{Kmol}^{-1}$ at $35 \mathrm{~K}$, consistent with dominant antiferromagnetic exchange interactions within complex 19. Below $25 \mathrm{~K}$, the $\chi_{\mathrm{M}} T$ value decreases steeply to reach $10.5 \mathrm{~cm}^{3} \mathrm{Kmol}^{-1}$ at $2 \mathrm{~K}$ indicating a non zero ground state spin for 19.

For 20, the $\chi_{\mathrm{M}} T$ value of $29.4 \mathrm{~cm}^{3} \mathrm{Kmol}^{-1}$ at $300 \mathrm{~K}$ is less than the expected value for two uncoupled $\mathrm{Mn}^{\mathrm{III}}(S=2, g=2.0)$ and two uncoupled Dy ${ }^{\mathrm{III}}\left({ }^{6} H_{15 / 2}, S=5 / 2, L=5\right)$ ions of 34.74 $\mathrm{cm}^{3} \mathrm{Kmol}^{-1}$, and the $\chi_{\mathrm{M}} T$ decrease steadily with decreasing temperature to reach 25.1 $\mathrm{cm}^{3} \mathrm{Kmol}^{-1}$ at $60 \mathrm{~K}$; the behavior being consistent with antiferromagnetic exchange interactions within 20 . After $60 \mathrm{~K}$, the $\chi_{\mathrm{M}} T$ value decreases steeply to reach $13.8 \mathrm{~cm}^{3} \mathrm{Kmol}^{-1}$ at $2 \mathrm{~K}$ indicating a relatively large ground state spin compare to complex $\mathbf{2 0}$.

The molar dc magnetic susceptibility values of the above-mentioned complexes are indicative of the contribution of the lanthanide ion to the net experimental magnetic susceptibility observed and consequently the resulting magnetic properties of these complexes (vide infra). This is hypothesized because all of these complexes have two $\mathrm{Mn}^{\mathrm{III}}$ centers, in addition to the $\mathrm{Ln}$ ions, and the free-ion $\chi_{\mathrm{M}} T$ value (in $\mathrm{cm}^{3} \mathrm{Kmol}^{-1}$ ) of individual $\mathrm{Ln}^{3+}$ ions in decreasing order is Dy (14.17) > Tb (11.82) > Gd (7.88). Accordingly, the magnitude of the $\chi_{\mathrm{M}} T$ with decreasing temperatures and even at $300 \mathrm{~K}$, follows the same descending pattern, i.e., 20 > 19 $>\mathbf{1 8}$, as that of the free-ion $\chi_{\mathrm{M}} T$ values of the constituent $\mathrm{Ln}^{3+}$ ions of these complexes. Thus, both the data are satisfyingly consistent and the overall nature of the plots for all the complexes is very similar as can be seen in Figure 7.10. The calculation of individual pairwise exchange parameters $\left(J_{\mathrm{ij}}\right)$ within the $\mathrm{Mn}_{2} \mathrm{Ln}_{2}$ molecules is in progress.

The field dependence of the magnetisation $(0-5 \mathrm{~T})$ at $1.8 \mathrm{~K}$ (Figure 7.11) for each of the compounds 18-20 shows a relative increase of the magnetisation at low fields, followed by an almost linear increase without clear saturation up to $5 \mathrm{~T}$, where it reaches a value of $14.96 \mu_{\mathrm{B}}$ 
for $\mathbf{1 8}, 12.1 \mu_{\mathrm{B}}$ for $\mathbf{1 9}$, and $12.9 \mu_{\mathrm{B}}$ for $\mathbf{2 0}$ indicating the presence of non zero ground state in these compounds, probably the ground state $S_{\mathrm{T}}$ is $\sim 7$, but to get more precise information about spin ground state $S$ and magnetic anisotropy term $D$, the fitting of the single crstal magnetisation measurement should also be done, that are in progress.
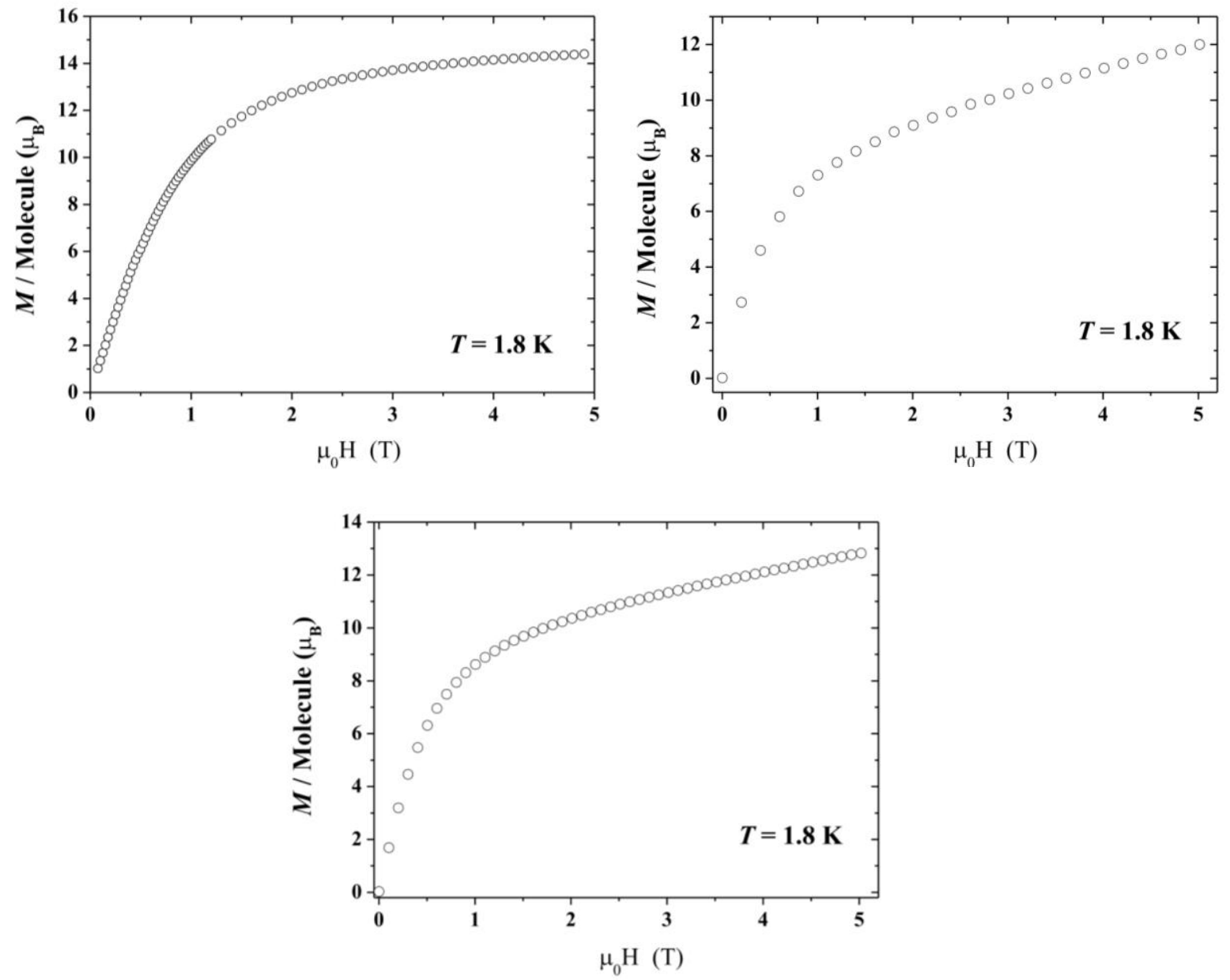

Figure 7.11. Plots of magnetisation $M /$ Molecule $\left(\mu_{\mathrm{B}}\right)$ versus applied field $H$ for $\mathbf{1 8}$ (top-left), $\mathbf{1 9}$ (top-right), and 20 (bottom) at $1.8 \mathrm{~K}$.

As a result of non zero ground state, and anisotropy present in these compounds, the alternating current (ac) susceptibility measurements were checked under zero dc field in the frequency range $10-1488 \mathrm{~Hz}$ at temperatures (Figure 7.12). In all cases in-phase $\left(m^{\prime}\right)$ value gradually increases with decreasing temperature, indicate the population of low-lying excited states. There is no any frequency dependence in-phase signal observed at low temperature (Figure 7.12). 

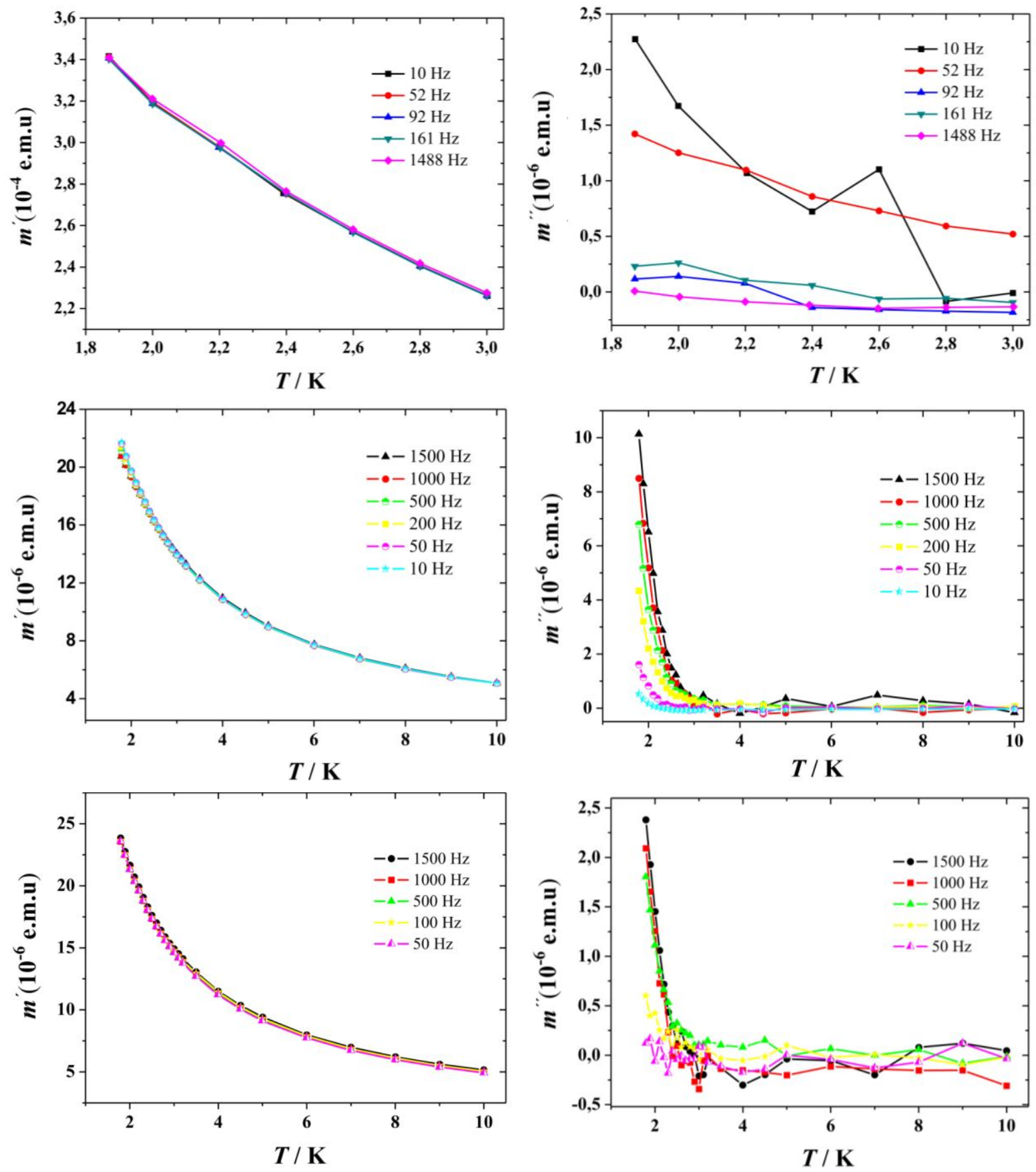

Figure 7.12. Plots of in-phase $\left(m^{\prime}\right.$, left $)$ and the out-of-phase $\left(m^{\prime \prime}\right.$, right $)$ ac susceptibilities versus temperature for complex 18 (top), 19 (middle), and 20 (bottom).

On the otherhand frequency dependence out-of-phase signals are clearly detected below $3 \mathrm{~K}$ for $\mathbf{1 8}$, and below $4 \mathrm{~K}$ for $\mathbf{1 9}$ and $\mathbf{2 0}$ indicating that these compounds exhibit slow relaxation of the magnetization. Thus these complexes might be SMMs.

To confirm further the SMM properties of $\mathbf{1 8}$ we used our home-made micro-Hall-bar magnetometer $^{77}$ in order to perform low-temperature single-crystal magnetization 
measurements. Magnetization vs. field hysteresis, the diagnostic property of a magnet, was indeed observed below $2.1 \mathrm{~K}$ at a field sweep rate of $50 \mathrm{mTs}^{-1}$ (Figure 7.13).
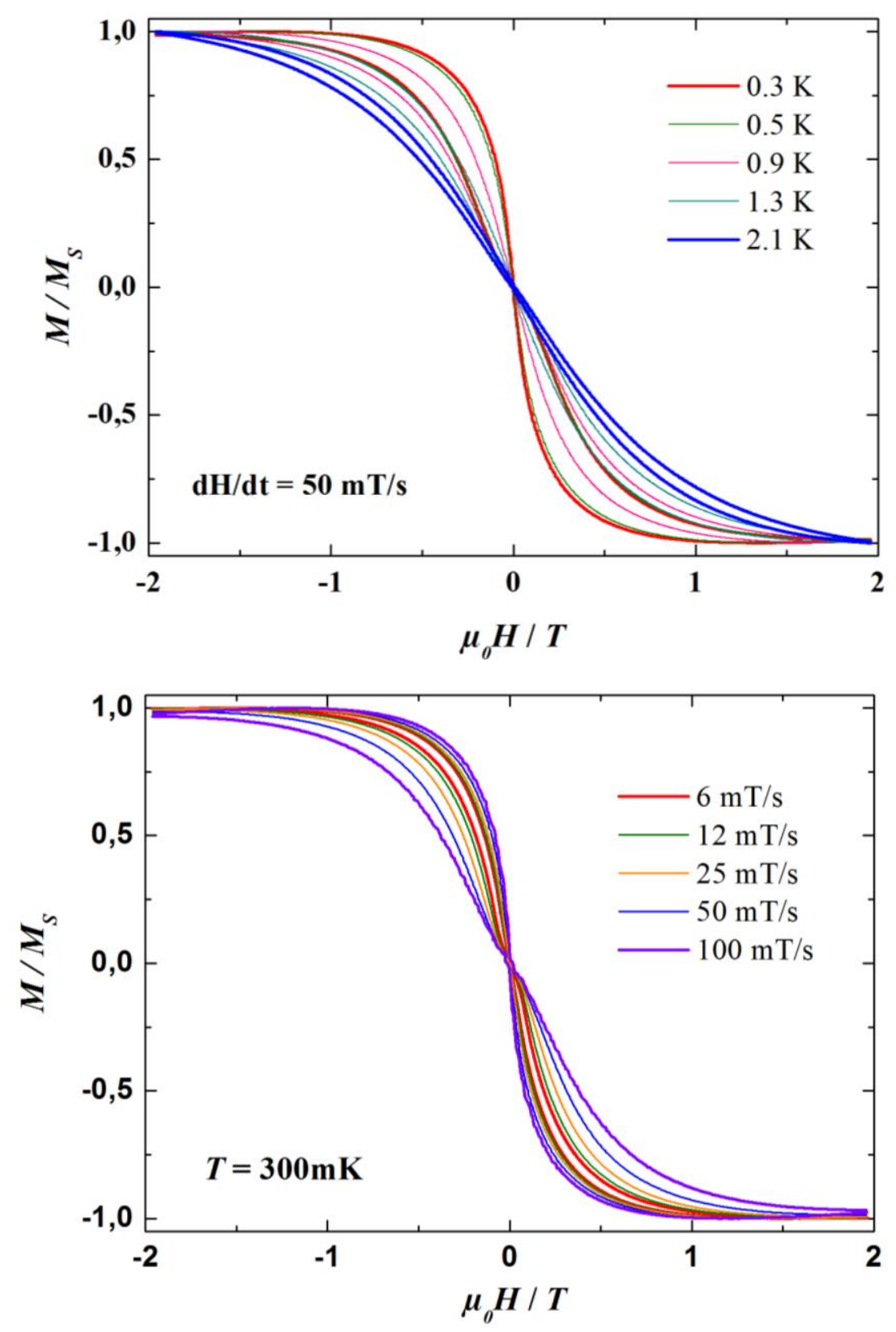

Figure 7.13. Magnetization $(M)$ versus applied DC field $H$ hysteresis loops for single crystals of $\mathbf{1 8}$. The resulting loops are shown at the indicated temperatures (top), and indicated field sweep rates (bottom). The magnetisation is normalised to its saturation value $M_{\mathrm{s}}$.

The hysteresis loops exhibit increasing coercivity with decreasing temperature at a constant sweep rate (Figure 7.13, top) and increasing coercivity with increasing field sweep rate at a constant temperature (Figure 7.13, bottom). This is as expected for the superparamagnet-like 
properties of a SMM. Thus it is confirmed that complex $\mathbf{1 8}$ to be a new addition to the family of SMMs. The blocking temperature $\left(T_{\mathrm{b}}\right)$ is $\sim 2.1 \mathrm{~K}$, above which there is no hysteresis, i.e., the spin relaxes faster to equilibrium than the time scale of the hysteresis loop measurements. It is worth to mention that to the best of our knowledge this is the highest blocking temperature $\left(T_{\mathrm{b}}=2.1 \mathrm{~K}\right)$ so far in Mn-Ln chemistry array till to date. ${ }^{89 \mathrm{~b}}$

\subsection{Conclusions}

In this chapter, we have shown that the versatile $\mathbf{H}_{2} \mathbf{L}^{9}$ ligand provided access to new mixed valence trinuclear, and octanuclear clusters of $\mathrm{Mn}(\mathrm{II} / \mathrm{III})$ and assembled a new family of $3 d-4 f$ compounds containing $\mathrm{Mn}^{\mathrm{III}}$. The trinuclear mixed valent $\mathrm{Mn}(\mathrm{II} / \mathrm{III})$ complex showed slowrelaxation behavior at low temperature, suggests a new SMMs. All the Mn-Ln compounds show single-molecule magnet behavior that is tuned by the choice of the lanthanide ion, in particular for compound 18, the highest blocking temperature reported to date for a heterometallic Mn-Ln SMMs. The series of molecular magnets reported in this chapter highlight the importance of simple chemical approaches to obtain targeted series of pure molecular species showing interesting magnetic properties. 


\section{Chapter 8}

\section{New Binucleating Pyrazolate Ligands with Phenol Side arms- Potential Scaffolds for Bimetallic Multiradical Chemistry}

\subsection{Introduction}

The presence of amino acid radicals like tyrosine-based radicals ${ }^{93}$ as an electron reservoir in redox enzymes that activate dioxygen has stimulated model studies ${ }^{94}$ with structurally defined small-molecule analogues of metallobiomolecules to elucidate the molecular basis of their reactivity. These model compounds have often the potential for being developed into new catalysts for oxygen activation that are capable of unusual substrate oxidations. In this regard the fungal enzyme galactose oxidase (GO), ${ }^{95}$ whose active site contains one copper ion coordinated to a tyrosyl (phenoxyl) radical and catalyzes the two-proton and two-electron oxidation of primary alcohols to the corresponding aldehydes in the presence of dioxygen, has stimulated important developments in the field of aerial oxidation of alcohols catalyzed by copper(II)-containing GO analogues.

The crystal structure ${ }^{95 a, b}$ of GO shows a distorted square-pyramidal copper site with two histidine nitrogen and two oxygen (from one axial and one equatorial tyrosine) donor atoms, plus an exogenous water in the equatorial site, which is also the binding site for the substrate (Figure 8.1). The copper(II) center with tyrosine (Tyr 272) radical in the equatorial position which is responsible for hydrogen atom abstraction from the substrate is the active form of Galactose Oxidase. GO thus contains two electron redox centers: 1e- per copper, shuttling between $\mathrm{Cu}(\mathrm{I})$ and $\mathrm{Cu}(\mathrm{II})$, and 1e- from tyrosine radical.

To model the active site of GO, several biomimetic approaches have been developed during the last decade. ${ }^{94}$ Consequently, a large number of complexes of the first-row transition metals with noninnocent ligands have been characterized. Tetradentate salen type ligands have been commonly used to provide a coordination sphere similar to that of the enzyme. Particular mononuclear phenolatocopper (II) complexes with salen type ligands can undergo a one-electron oxidation into magnetically interacting phenoxyl copper(II) complexes. ${ }^{36 \mathrm{a}-\mathrm{c}}$ The localization of the oxidation site in such complexes could, however, lead to discrepancies as electrons can be removed either from the metal center (leading to an $\mathbf{M}^{(\mathrm{n}+1)+}$ closed shell ligand) or from the ligand (leading to an $\mathrm{M}^{\mathrm{n}+}$ open shell ligand), and this situation could become even more complicated when valence tautomerism exists between these two forms, as 
in nickel porphyrins: ${ }^{96}$ in this case, the two forms the $\mathrm{M}^{(\mathrm{n}+1)+}$ closed-shell porphyrin and the $\mathrm{M}^{\mathrm{n}+}$ open-shell porphyrin are in equilibrium.

(A)

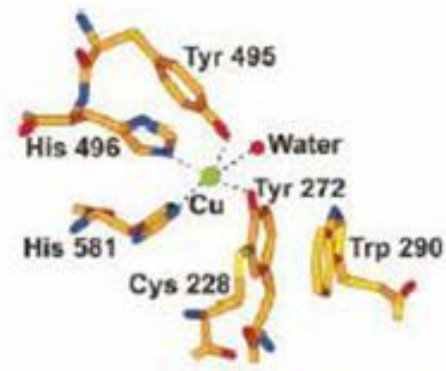

(C)
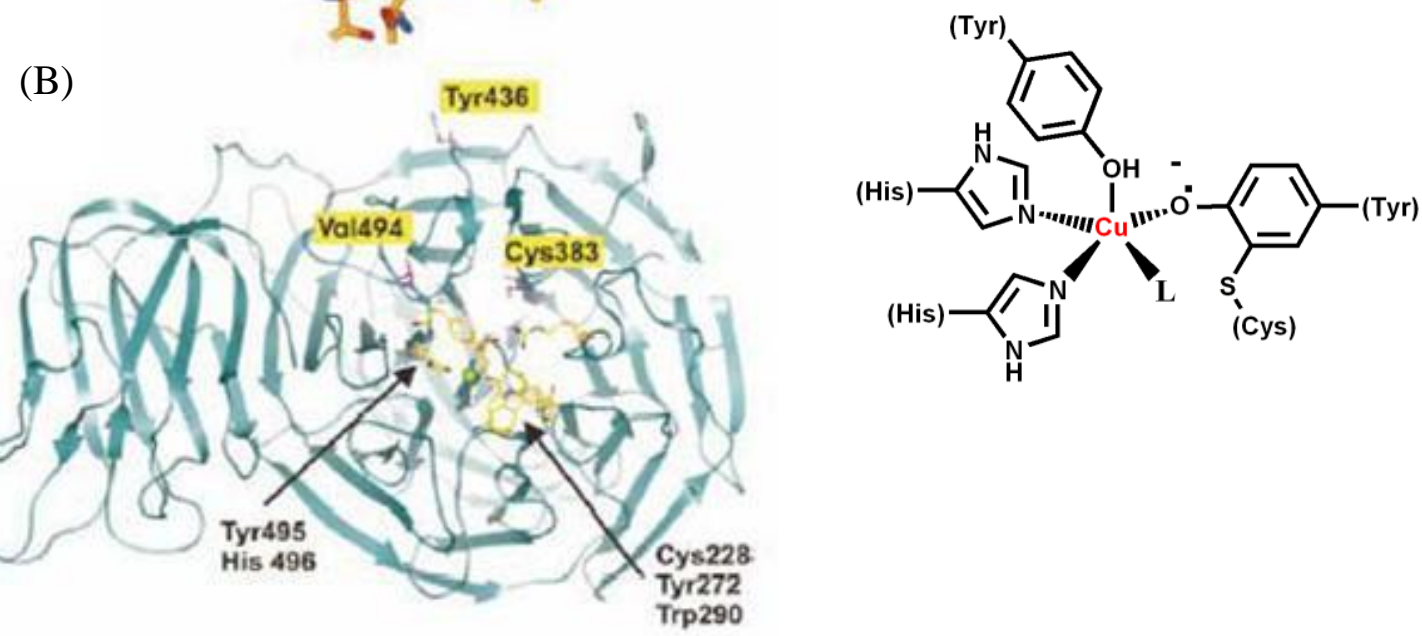

Figure 8.1. Structure of GOase. (A) The active site showing the copper ligands, the thioether bond between Tyr272 and Cys228, and the stacking interaction with Trp290. (B) An overview of the GOase monomer showing domains 1 and 2 and showing the locations of the residues Cys383, Tyr436 and Val 494 (shown in mauve and highlighted by yellow labels), which form the basis of the present study. The active site residues are shown in atom colouring and are labelled by arrows. Non-covalent bond interactions are shown as dotted lines.11-12 (C) Schematic diagram of Goase (active form), $\mathrm{L}=\mathrm{H}_{2} \mathrm{O}$.

In this regard, bimetallic transition metal complexes have received a lot of attention ${ }^{97}$ mainly due to the increasing interest in cooperative effects between individual metal centers. Here we designed new pyrazolate-based bridging ligand with two phenol side arms in the 3- and 5positions of the heterocycle, the anionic pyrazolate has a high tendency to span two metal ions, and individual coordination spheres as well as metal-metal separations can be tuned by appropriate alterations of the appended chelate substituents. Type B bimetallic complexes with pyrazolate-bridging ligands can be described as an extended version of the metal-salen skeleton (Chart 8.1). Preferred metal-metal separations range from 4.1-4.5 $\AA$, depending on the secondary bridging moiety of the metal ions. Mononuclear salen type complexes A with $\mathrm{Zn}^{2+}, \mathrm{Cu}^{2+}, \mathrm{Ni}^{2+}$ ions have proven highly successful for metal-radical chemistry and reactivities of primary alcohols oxidation. ${ }^{98,36 a-c, 99}$ 


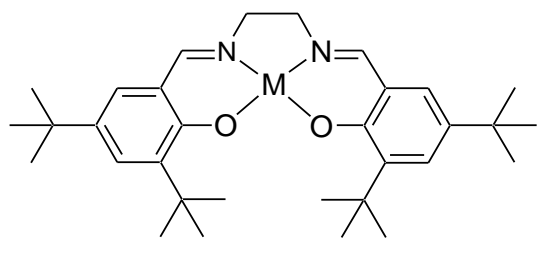

A

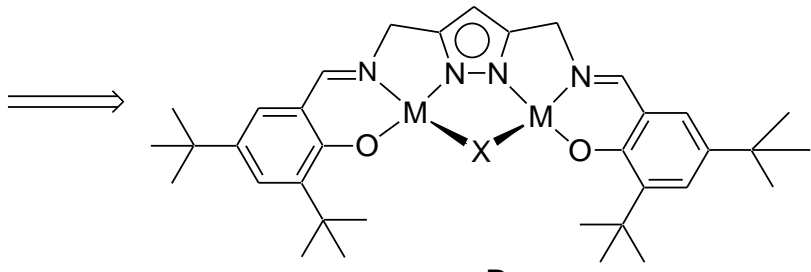

B

\section{Chart 8.1.}

For their bimetallic analogues $\mathbf{B}$, a similarly rich bio- mimetic chemistry is just emerging and offers great prospects due to the potential of the highly preorganized proximate metal ions to work in concert during substrate transformations. ${ }^{100,101,102}$ Here we have synthesized a new pyrazolate-based bridging ligand with two phenol side arms $\left(\mathbf{H}_{\mathbf{3}} \mathbf{L}^{\mathbf{1 2}}\right)$, where $o$-, and $p$-position of the phenolate contains $t$-butyl group (that can prevent the radical initiated decomposition), and studied its binuclear complexes with $\mathrm{Cu}^{2+}, \mathrm{Ni}^{2+}$ and tetranuclear complex with $\mathrm{Zn}^{2+}$ in metal-radical chemistry.

\subsection{Ligand Synthesis}

The new ligand $\mathbf{H}_{\mathbf{3}} \mathbf{L}^{\mathbf{1 2}}$ was synthesized in a multistep sequence starting from 3,5-dimethyl pyrazole (Scheme 8.1). The intermediate 3,5-bis(chloromethyl)-1-(tetrahydropyran-2-yl)-1H pyrazole was obtained in 5 steps according to the literature method, ${ }^{103 a, b}$ and was then treated with phthalimide in dry DMF, followed by a reaction with hydrazine hydrate, ${ }^{103 c}$ and subsequently deprotection of the THP group by hydrochloric acid, gave 3,5-bis(aminoethyl) pyrazol dihydrochloride as a desired starting precursor. Final step is the condensation of 2 equivalent 3,5-di-tert-butyl-salicylaldehyde with 1 equivalent neutralised 3,5-bis(aminoethyl) pyrazole dihydrochloride in methanol afforded the ligand $\mathbf{H}_{3} \mathbf{L}^{\mathbf{1 2}}$ as yellowish solid in $98 \%$ yield. It was characterized by NMR $\left({ }^{1} \mathrm{H},{ }^{13} \mathrm{C}\right)$ spectroscopy, mass spectrometry and microanalysis. 


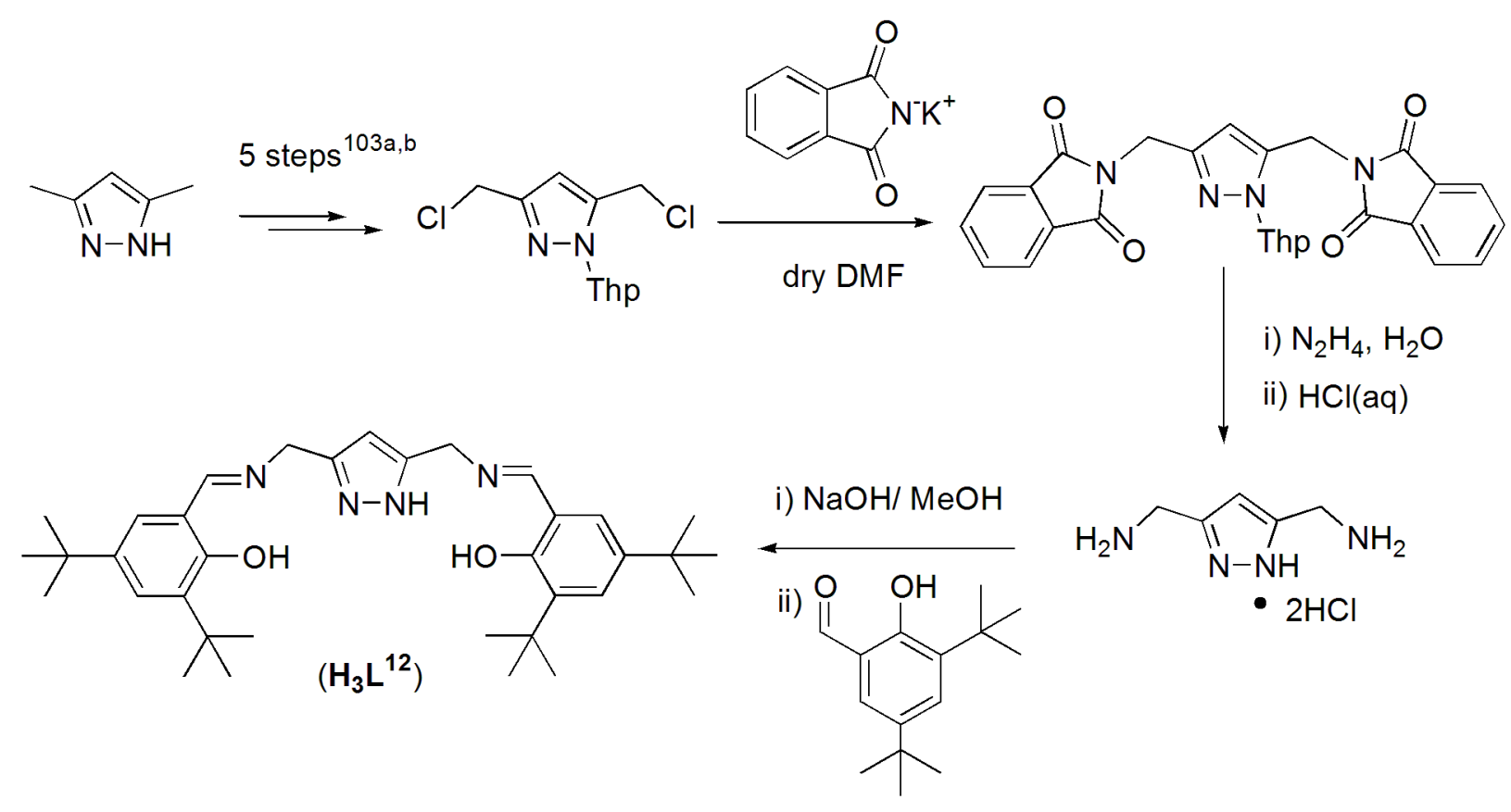

Scheme 8.1. General Synthesis of the Novel Ligand $\mathbf{H}_{3} \mathbf{L}^{\mathbf{1 2}}$

\subsection{Synthesis and Molecular Structure of Metal Complexes}

The reaction of $\mathbf{H}_{3} \mathbf{L}^{\mathbf{1 2}}$ with 2 equivalents of $\mathrm{Cu}\left(\mathrm{BF}_{4}\right)_{2} \cdot 4 \mathrm{H}_{2} \mathrm{O}$ and 3 equiv. of $\mathrm{NaOMe}$ in methanol yielded a deep green solution, and slow evaporation of the resulting solution afforded needle shaped green crystal of $\left[\left(\mathrm{L}^{12}\right) \mathrm{Cu}_{2}(\mu-\mathrm{MeOH} \cdots \mathrm{OH})\right] \cdot 2.5 \mathrm{MeOH} 21$, which was suitable for X-ray crystallographic analysis; Figure 8.2 shows the obtained molecular structure. In the resulting complex 21, two copper(II) ions are hosted in the two ligand compartments, ligated by the phenolato-O, the imine-N, and the adjacent pyrazole- $\mathrm{N}$, and both copper(II) ions are found in roughly square planar environment.

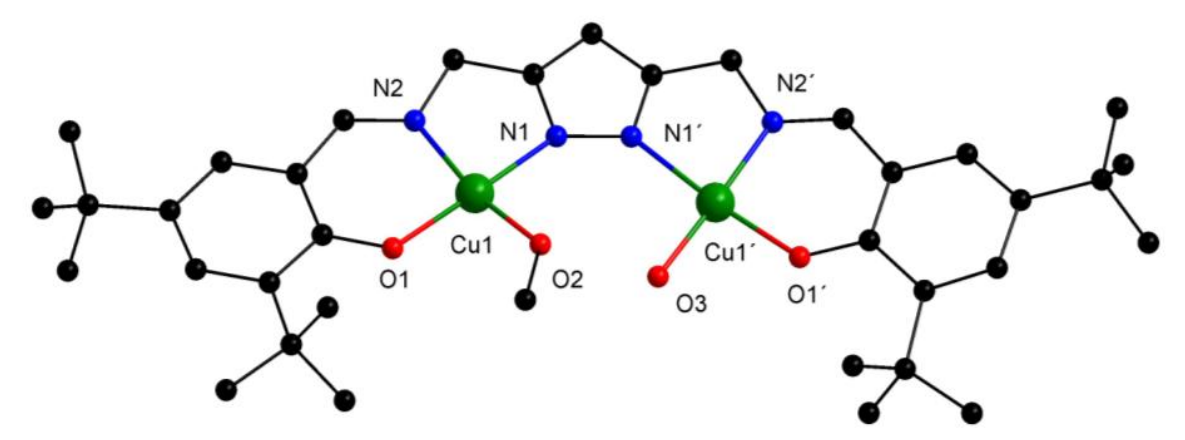

Figure 8.2. View of the molecular structure of $\left[\left(\mathrm{L}^{12}\right) \mathrm{Cu}_{2}\left(\mu-\mathrm{MeOH}{ }^{\cdots} \mathrm{OH}\right)\right] \cdot 2.5 \mathrm{MeOH}$ 21. Solvent molecules, hydrogen atoms are omitted for clarity. Colour code. $\mathrm{Cu}$ green, $\mathrm{O}$ red, $\mathrm{N}$ blue, $\mathrm{C}$ black. 
The methanol, and water solvent ligands are located in the copper(II) coordination planes and, form intramolecular hydrogen bond [O $\cdots \mathrm{O}$ distance 2.41 $\AA$ ] (Chart 8.2). The inter-atomic distance between the two $\mathrm{Cu}^{2+}$ ions is $4.43 \AA$.

The bond lengths Cu1-O1 (or Cu1'-O1'), Cu1-O2, Cu1'-O3 (1.9055(3), 1.9842(6), 1.8633(9) $\AA$ respectively) are shorter than Cu1-N1, Cu1-N2 (or Cu1'-N1', Cu1'-N2') [1.9978(4), 1.9262(4) Å respectively]. Bond angles O1-Cu1-N2, N1-Cu1-N2, N1-Cu1-O2, O1-Cu1-O2

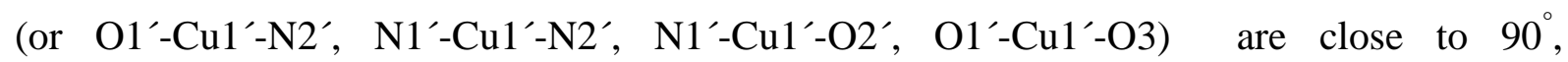
$\left(92.897(1)^{\circ}, 83.536(2)^{\circ}, 93.172(2)^{\circ}, 91.457(2)^{\circ}\right.$ respectivly), which suggesting that both ions are in roughly square planar environments. It should be noted that the two planes of $\mathrm{Cu} 1, \mathrm{O} 1$, $\mathrm{O} 2, \mathrm{~N} 1, \mathrm{~N} 2$ and $\mathrm{Cu}^{\prime}, \mathrm{O}^{\prime}, \mathrm{O} 3, \mathrm{~N}^{\prime}, \mathrm{N} 2^{\prime}$ are slightly twisted by $12.3^{\circ}$.

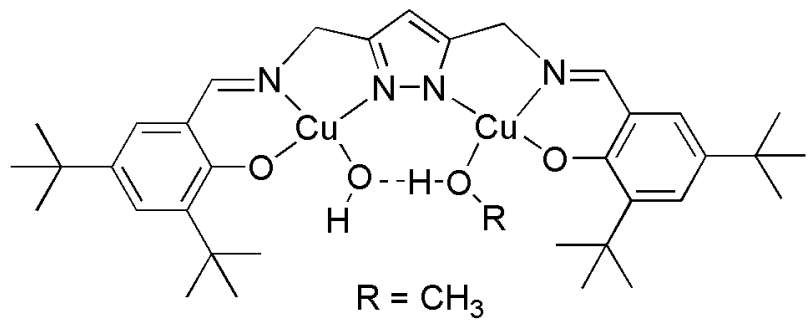

Chart 8.2. Schematic diagram of compound 21.

Upon reaction of $\mathbf{H}_{3} \mathbf{L}^{\mathbf{1 2}}$ with 2 equiv. of $\mathrm{Cu}(\mathrm{OAc})_{2} \cdot \mathrm{H}_{2} \mathrm{O}$ in methanol at room temperature afforded a deep green precipitate immediately, and then recrystallised by $\mathrm{CHCl}_{3}$-hexane mixture yielded needle shaped green crystals of $\left[\left(\mathrm{L}^{12}\right) \mathrm{Cu}_{2}(\mu-\mathrm{OAc})\right] \cdot 1.33 \mathrm{CHCl}_{3} \mathbf{2 2}$, and in similarway using $\mathrm{Ni}(\mathrm{OAc})_{2} \cdot 4 \mathrm{H}_{2} \mathrm{O}$ instead of $\mathrm{Cu}(\mathrm{OAc})_{2} \cdot \mathrm{H}_{2} \mathrm{O}$, yellow crystals of compound $\left[\left(\mathrm{L}^{12}\right) \mathrm{Ni}_{2}(\mu-\mathrm{OAc})\right] \cdot 1.33 \mathrm{CHCl}_{3} \mathbf{2 3}$, was isolated in quantitavie yield. A crystallographic analysis afforded the molecular structures as depicted in Figure 8.3, and 8.4 respectively. In both cases, two metal ions are resided in the two ligand compartments as like before and those are bridged by acetate form a square planar complex as anticipated. The inter-atomic distances between the two metal ions are $4.12 \AA$ for complex 22, and $4.17 \AA$ for complex 23. The bond lengths Cu1-O1, Cu1-N3, Cu1-O3 (1.8729(1), 1.9434(3), 1.9574(2) Å respectively) are slightly shorter than $\mathrm{Cu} 2-\mathrm{O} 2, \mathrm{Cu} 2-\mathrm{N} 4, \mathrm{Cu} 2-\mathrm{O} 4$ [1.893(2), 1.9450(2), 1.9629(1) $\AA$ respectively]. In contrast the bond length of Cu1-N1 (1.9321(2) $\AA$ ) is longer than Cu2-N2 (1.8633(9) $\AA$ ), clearly says that co-ordination environment of the both copper ions (Cu1 and $\mathrm{Cu} 2)$ are not identical in 22. The bond angles O1-Cu1-N3, O1-Cu1-O3, N1-Cu1-N3, N1-Cu1$\mathrm{O} 3\left(93.158(8)^{\circ}, 87.789(7)^{\circ}, 82.621(9)^{\circ}, 98.601(8)^{\circ}\right.$ respectively) or O2-Cu2-N4, O2-Cu2-O4, N2-Cu2-N4, N2-Cu2-O4 (93.326(8) $, 96.696(8)^{\circ}, 82.38(8)^{\circ}, 97.678(2)^{\circ}$ respectively), differes 
slightly from $90^{\circ}$, suggesting that both ions are in roughly square planar environments and the angles between the two planes of $\mathrm{Cu} 1, \mathrm{O} 1, \mathrm{~N} 1, \mathrm{~N} 3$ and $\mathrm{Cu} 2, \mathrm{O} 2, \mathrm{~N} 2, \mathrm{~N} 4$ is $18^{\circ}$ implies that both copper atoms are not coplanar, slightly twisted by bridging ligand acetate.

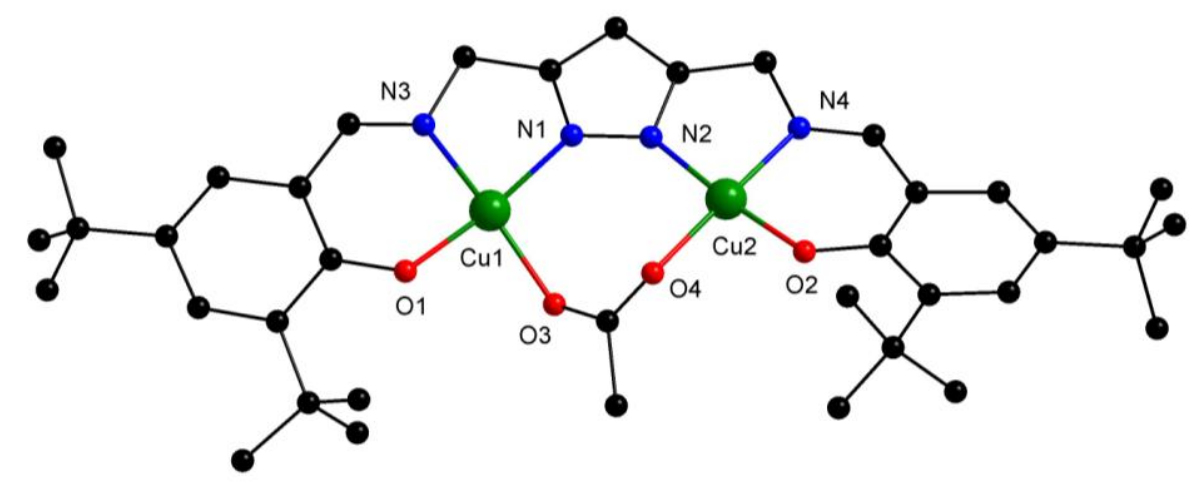

Figure 8.3. View of the molecular structure of $\left[\left(\mathrm{L}^{12}\right) \mathrm{Cu}_{2}(\mu-\mathrm{OAc})\right] \cdot 1.33 \mathrm{CHCl}_{3}$ 22. Solvent molecules, hydrogen atoms are omitted for clarity. Colour code. $\mathrm{Cu}$ green, $\mathrm{O}$ red, $\mathrm{N}$ blue, $\mathrm{C}$ black.

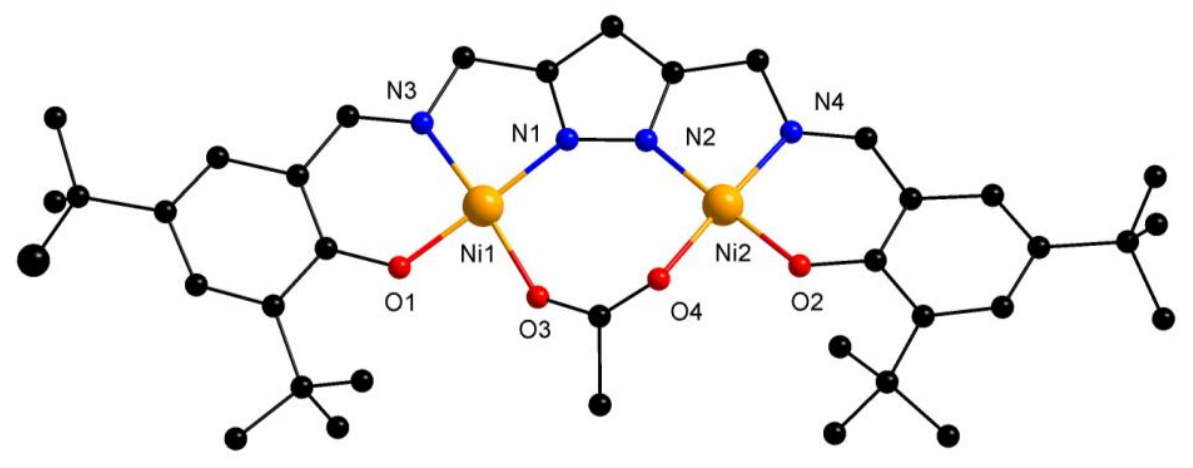

Figure 8.4. View of the molecular structure of $\left[\left(\mathrm{L}^{12}\right) \mathrm{Ni}_{2}(\mu-\mathrm{OAc})\right] \cdot 1.33 \mathrm{CHCl}_{3}$ 23. Solvent molecules, hydrogen atoms are omitted for clarity. Colour code. Ni yellow, $\mathrm{O}$ red, $\mathrm{N}$ blue, $\mathrm{C}$ black.

The geometry of the both nickel atoms is roughly square planar in 23: The bond lengths Ni1O1 (or Ni2-O2), Ni1-O3 (or Ni2-O4) (1.83(1), 1.87(1) ^ respectively) are slightly shorter than Ni1-N1 (or Ni2-N2), Ni1-N3 (or Ni2-N4) lengths (1.88(1), 1.84(1) ̊ respectively). The bond angles O1-Ni1-N3, O1-Ni1-O3, N1-Ni1-N3, and N1-Ni1-O3 or O2-Ni2-N4, O2-Ni2$\mathrm{O} 4, \mathrm{~N} 2-\mathrm{Ni} 2-\mathrm{N} 4$, and N2-Ni2-O4 are differ slightly from $90^{\circ}\left(93.04(5)^{\circ}, 82.585(5)^{\circ}, 85.173(6)^{\circ}\right.$, $99.294(5)^{\circ}$ respectively), or $\left(93.796(5)^{\circ}, 82.357(6)^{\circ}, 84.487(6)^{\circ}, 99.351(5)^{\circ}\right)$. Both nickel atoms are not coplanar, those are slightly twisted by bridging ligand acetate as like before (the angle between the two plane of Ni1, N1, N3, O1, and Ni2, N2, N4, O2 is $11.50^{\circ}$ ). The inter- 
atomic distance between the two nickel atoms is $4.12 \AA$. Selected interatomic distances, and bond angles of compounds $\mathbf{2 1 - 2 3}$ are given in Table 8.1.

Table 8.1. Selected bond lengths $(\AA)$ and angles $\left({ }^{\circ}\right)$ for 21-23.

\begin{tabular}{|c|c|}
\hline \multicolumn{2}{|c|}{21} \\
\hline Cu1-O1 1.9055(3) & $\mathrm{Cu}^{\prime}-\mathrm{O} 1^{\prime} 1.9055(3)$ \\
\hline $\mathrm{Cu} 1-\mathrm{O} 2$ 1.9842(6) & Cu1'-O3 1.8633(9) \\
\hline Cu1-N1 1.9978(4) & Cu1'-N1' 1.9978(4) \\
\hline Cu1-N2 1.9262(4) & $\mathrm{Cu}^{\prime}-\mathrm{N} 2^{\prime} 1.9262(4)$ \\
\hline O1-Cu1-O2 91.457(2) & $\mathrm{O}^{\prime}-\mathrm{Cu} 1^{\prime}-\mathrm{O} 2^{\prime} 91.457(2)$ \\
\hline O1-Cu1-N2 92.897(1) & O1'-Cu1'-N2' 92.897(1) \\
\hline N1-Cu1-N2 83.536(2) & $\mathrm{N} 1^{\prime}-\mathrm{Cu} 1^{\prime}-\mathrm{N} 2^{\prime} 83.536(2)$ \\
\hline N1-Cu1-O2 93.172(2) & $\mathrm{N}^{\prime \prime}-\mathrm{Cu} 1^{\prime}-\mathrm{O} 2^{\prime} 93.172(2)$ \\
\hline \multicolumn{2}{|r|}{22} \\
\hline Cu1-O1 1.8729(1) & $\mathrm{Cu} 2-\mathrm{O} 2$ 1.893(2) \\
\hline Cu1-O3 1.9574(2) & Cu2-O4 1.9629(1) \\
\hline Cu1-N1 1.9321(2) & Cu2-N2 1.8633(9) \\
\hline Cu1-N3 1.9434(3) & $\mathrm{Cu} 2-\mathrm{N} 4$ 1.9450(2) \\
\hline O1-Cu1-O3 87.789(7) & O2-Cu2-O4 96.696(8) \\
\hline O1-Cu1-N3 93.158(8) & O2-Cu2-N4 93.326(8) \\
\hline N1-Cu1-N3 82.621(9) & N2-Cu2-N4 82.38(8) \\
\hline N1-Cu1-O3 98.601(8) & N2-Cu2-O4 97.678(2) \\
\hline \multicolumn{2}{|r|}{23} \\
\hline Ni1-O1 1.8336(1) & Ni2-O2 1.8379(1) \\
\hline Ni1-O3 1.8708(1) & Ni2-O4 1.8794(1) \\
\hline Ni1-N1 1.8869(1) & Ni1- Ni2 4.1742 (2) \\
\hline Ni1-N3 1.8436(0) & Ni2-N4 1.8463(1) \\
\hline O1-Ni1-O3 82.585(5) & O2-Ni2-O4 82.357(6) \\
\hline O1-Ni1-N3 93.040(5) & O2-Ni2-N4 93.796(5) \\
\hline N1-Ni1-N3 85.173(6) & N2-Ni2-N4 84.487(6) \\
\hline N1-Ni1-O3 99.294(5) & N2-Ni2-O4 99.351(5) \\
\hline
\end{tabular}

The reaction of $\mathbf{H}_{\mathbf{3}} \mathbf{L}^{\mathbf{1 2}}$ with 2 equivalents of $\mathrm{Zn}\left(\mathrm{ClO}_{4}\right)_{2} \cdot 6 \mathrm{H}_{2} \mathrm{O}$, and 3 equivalents of $\mathrm{NaO}_{2} \mathrm{CPh}$ in methanol gave a light yellow precipitate immediately, and recrystallised by $\mathrm{CHCl}_{3}$-hexane mixture yielded yellow crystals of $\mathrm{Zn}_{4}\left(\mathrm{~L}^{12}\right)_{2}\left(\mathrm{H}_{2} \mathrm{~L}^{12}\right)_{2}$ 24. However due to low quality of the crystals the molecular structure of this compound could not be determined from X-ray crystallographic analysis. But based on elemental analysis, mass spectrometry, and NMR $\left({ }^{1} \mathrm{H}\right.$, ${ }^{13} \mathrm{C}$ ) spectroscopy proposed structure of compound $\mathbf{2 4}$ is [2×2]-grid structure composed of four metal ions and four ligand strands in which two fully deprotonated rigid ligands $\left(\mathrm{L}^{12}\right)^{3-}$ and two partially deprotonated flexible ligand $\left(\mathrm{H}_{2} \mathrm{~L}^{12}\right)^{-}$(see Chart 8.3). 


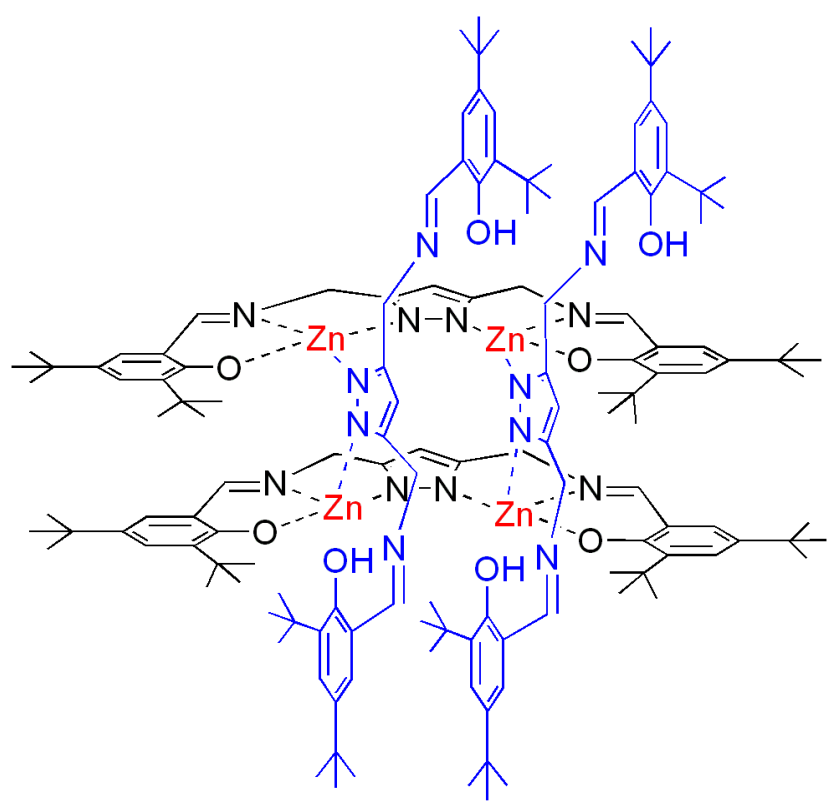

Chart 8.3. Proposed structure of compound $\mathrm{Zn}_{4}\left(\mathrm{~L}^{12}\right)_{2}\left(\mathrm{H}_{2} \mathrm{~L}^{12}\right)_{2} 24$.

\subsection{Magnetic Properties of $\mathrm{Cu}(\mathrm{II})$ complexes}

In order to gain the electronic structure of the copper(II) complexes with different secondary bridging ligands, compounds $\mathbf{2 1}$, and $\mathbf{2 2}$ were characterised by temperature dependent magnetic susceptibility measurement.

Magnetic susceptibility data were collected for two dinuclear copper (II) complex in the temperature range from 295 to $2.0 \mathrm{~K}$ in order to characterize the exchange coupling within the pyrazole core. No significant field dependence was observed when data were measured at applied fields of 0.2 and $0.5 \mathrm{~T}$. The temperature dependence of the molar magnetic susceptibility $\chi_{\mathrm{M}}$ and of the product $\chi_{\mathrm{M}} T$ is shown in Figure 8.5. The observed $\chi_{\mathrm{M}} T$ value at room temperature is $0.76 \mathrm{~cm}^{3} \mathrm{Kmol}^{-1}$ (corresponding $\mu_{\mathrm{eff}}=2.47 \mu_{B}$ ) for $\mathbf{2 1}$, and 0.94 $\mathrm{cm}^{3} \mathrm{Kmol}^{-1}$ (corresponding $\mu_{\text {eff }}=2.73 \mu_{\mathrm{B}}$ ) for 22, those are close to the theoretical value expected for two uncoupled copper (II) ions $\left(0.82 \mathrm{~cm}^{3} \mathrm{Kmol}^{-1}\right.$ or $\mu_{\mathrm{eff}}=2.57 \mu_{B}$ for $\left.g=2.1\right)$ for 21, and 22. In both cases, upon lowering the temperature, $\chi_{\mathrm{M}}$ goes through a broad maximum at $49 \mathrm{~K}$ for 21 and $52 \mathrm{~K}$ for $\mathbf{2 2}$, while $\chi_{\mathrm{M}} T$ drops and gradually tends to zero, which is indicative of significant antiferromagnetic coupling between the two copper(II) ions and an $S_{\mathrm{T}}$ $=0$ ground state. 

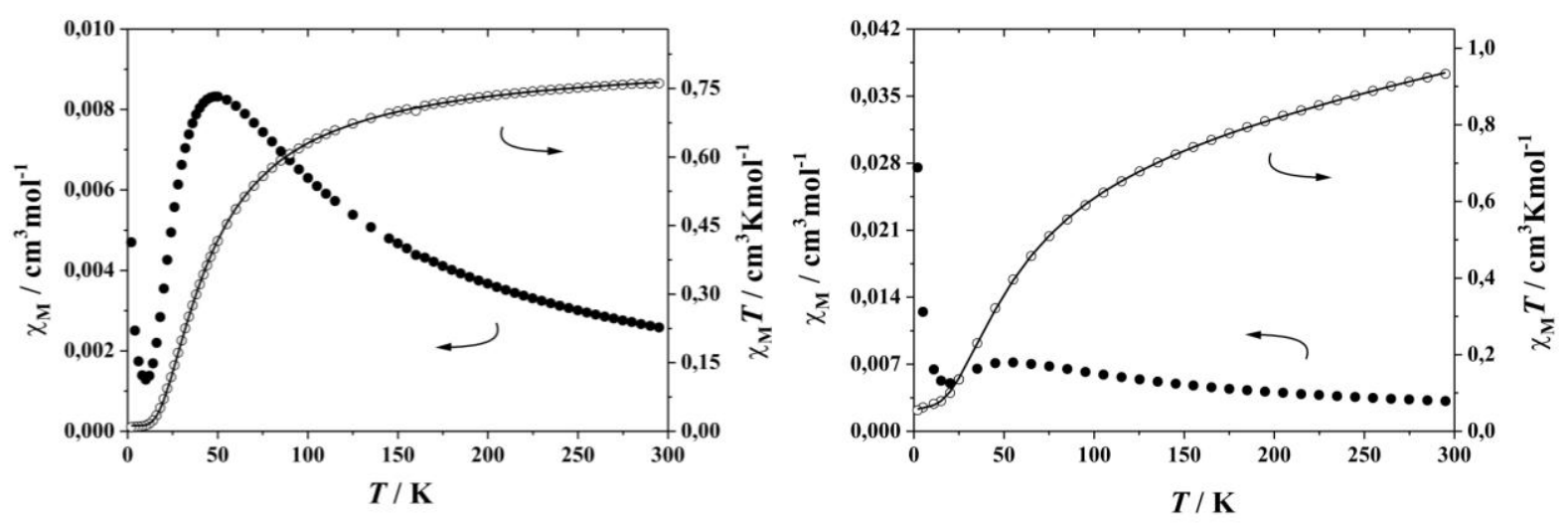

Figure 8.5. Plots of $\chi_{\mathrm{M}}$ (solid circles) and $\chi_{\mathrm{M}} T$ (open circles) versus temperature for 21 (left), and 22 (right) at $0.5 \mathrm{~T}$. The solid black lines represent the simulated curves.

Experimental data were simulated using a fitting procedure to the appropriate HeisenbergDirac-van-Vleck (HDvV) spin Hamiltonian for isotropic exchange coupling and Zeeman splitting (eq. 8.1) for both complexes.

$$
\hat{H}=-2 J \hat{S}_{1} \cdot \hat{S}_{2}+g \mu_{B}\left(\hat{S}_{1}+\hat{S}_{2}\right) B
$$

A Curie-Weiss-behaved paramagnetic impurity $(\rho)$ that presumably causes the increase of $\chi_{M}$ at very low temperatures and temperature-independent paramagnetism (TIP) were included according to $\chi_{\text {calc }}=(1-P I) \cdot \chi+P I \cdot \chi_{\text {mono }}+T I P$. Table 8.2. Summarizes the magnetic parameters obtained from best fit analyses.

Table 8.2: Best Fit Parameters of Magnetic Data Analysis for Complexes 21 and 22.

\begin{tabular}{|l|l|l|l|l|}
\hline complex & $g$ & $J\left[\mathrm{~cm}^{-1}\right]$ & $P I[\%]^{\mathrm{a}}$ & $T I P\left[10^{-6} \mathrm{~cm}^{3} \mathrm{~mol}^{-1}\right]$ \\
\hline $\mathbf{2 1}$ & 2.1 & -27.53 & 3.3 & 12.9 \\
\hline $\mathbf{2 2}$ & 2.1 & -36.88 & 15.0 & 868.9 \\
\hline
\end{tabular}

${ }^{\mathrm{a}} \mathrm{PI}$ indicate paramagnetic impurity.

The magnitude of the antiferromagnetic interaction of both compounds $\left(J=-27.53 \mathrm{~cm}^{-1}\right.$ for 21, and $J=-36.88 \mathrm{~cm}^{-1}$ for 22) implies that primary pathway for the magnetic excahnge intearaction is through the pyrazolate bridges. ${ }^{104 a}$ The coupling through the H-bonded $\mathrm{MeOH} \cdots \mathrm{OH}$ moeity in $\mathbf{2 1}$, and accetate moeity in $\mathbf{2 2}$ are negligable. The result for both complexes are lying well with reported $J$ values for many other known dicopper(II) complexes that are spanned by pyrazolate bridges feature square-planar or tetragonal metal 
ions where the magnetic $\mathrm{d}_{x 2-y 2}$ orbitals are located within the plane of the bridging heterocycles. ${ }^{104 \mathrm{~b}, \mathrm{c}}$

\subsection{Spectroscopy in Solution}

All new complexes were characterized by ESI mass spectrometry to clarify the stability of the complexes in solution. In order to elucidate the locous of the oxidised species of those complexes in solution, electrochemical, spectro-electrochemical experimets and EPRspectroscopy were performed.

8.5.1. Mass Spectrometry: ESI-MS spectra of $\mathrm{CHCl}_{3}$ solution of complexes 21 , and 22 shows a major peak at $\mathrm{m} / \mathrm{z} 682.9$, and 683.2 respectively, for the ion of $\left[\mathrm{L}^{12} \mathrm{Cu}_{2}\right]^{+}$, which implies that the binuclear entity is stable in solution. MALDI-MS spectrum of compound 24 in dithranol matrix displays prmoinent peak at $\mathrm{m} / \mathrm{z} 2488.3,1930.3,1305.7$, and 1242.6 characteristic for $\left[\mathrm{Zn}_{4}\left(\mathrm{~L}^{12}\right)_{2}\left(\mathrm{H}_{2} \mathrm{~L}^{12}\right)_{2}+\mathrm{H}^{+}\right], \quad\left[\mathrm{Zn}_{4}\left(\mathrm{~L}^{12}\right)_{2}\left(\mathrm{H}_{2} \mathrm{~L}^{12}\right)\right]^{+}, \quad\left[\mathrm{Zn}_{3}\left(\mathrm{~L}^{12}\right)_{2}-\mathrm{H}^{+}\right], \quad$ and $\left[\mathrm{Zn}_{2}\left(\mathrm{~L}^{12}\right)_{2}+\mathrm{H}^{+}\right]$respectively confirming that tetranuclear grid-type [2×2] complex stays intact in solution (Figure 8.6). These observations are important for analyzing the electrochemical and spectroelectrochemical experiments.

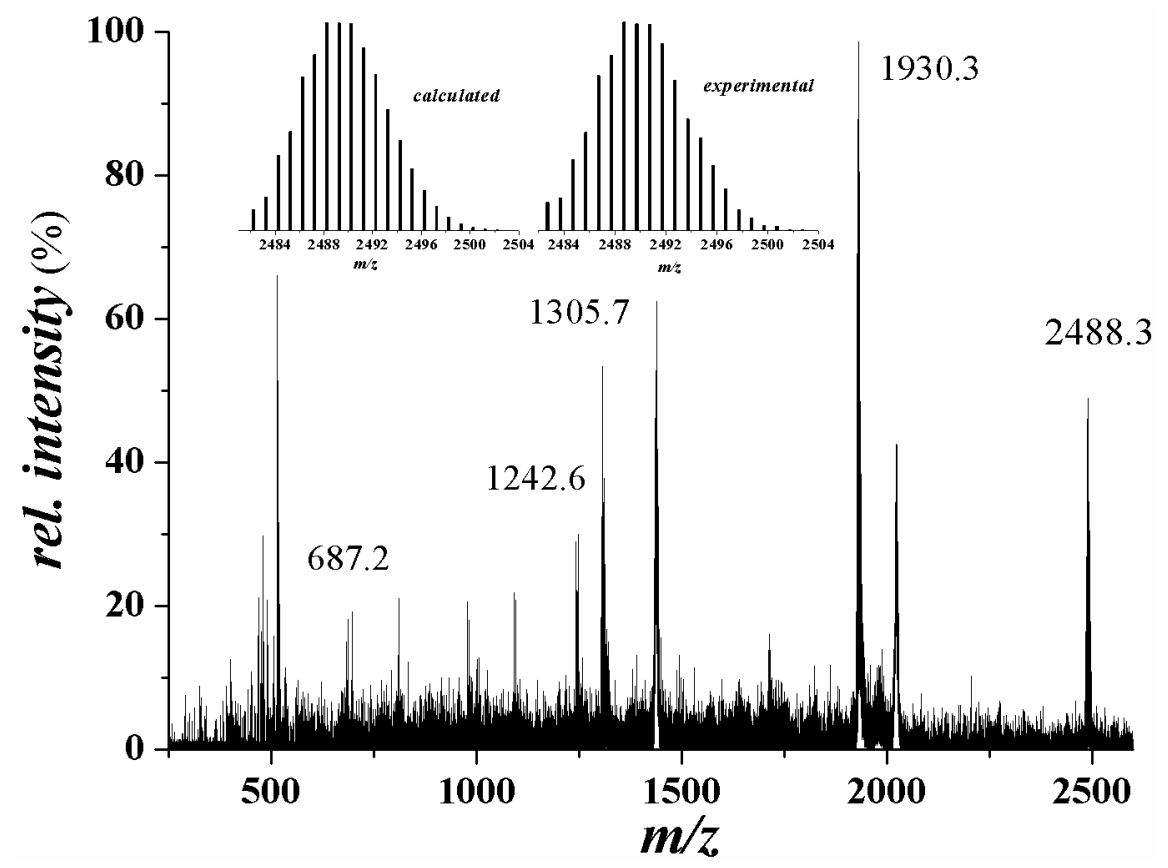

Figure 8.6. MALDI-MS spectrum of $\mathbf{2 4}$ in dirathanol (matrix). The inset shows the observed and calculated isotopic distribution pattern for the ions $\left[\mathrm{Zn}_{4}\left(\mathrm{~L}^{12}\right)_{2}\left(\mathrm{H}_{2} \mathrm{~L}^{12}\right)_{2}+\mathrm{H}^{+}\right]$. 
8.5.2. Electrochemistry: The redox properties of all complexes have been recorded in $\mathrm{CH}_{2} \mathrm{Cl}_{2}$, containing $0.10 \mathrm{M}\left[\mathrm{N}(n-\mathrm{Bu})_{4}\right] \mathrm{PF}_{6}$ as a supporting electrolyte. Small amount of ferrocene was added after the completion of each set of experiments as an internal standard, and all potentials are referenced versus the $\mathrm{Fc}^{+} / \mathrm{Fc}$ couple. Figure 8.7 (top) and Figure 8.8 displays cyclic voltammograms (CVs) of 22, 23, 21, and 24 respectively. Figure 8.7 (bottom) displays square-wave voltammograms (SWs) of $\mathbf{2 2}$ and $\mathbf{2 3}$ as an example for copper and nickel complexs. All redox potential obtained from the voltammograms is compiled in Table 8.2. The CV curves of $\mathbf{2 1 - 2 3}$ (Table 8.2) display two fully reversible close spaced oxidation waves $E_{1 / 2}{ }^{(0 /+)}=0.648$, and $E_{1 / 2}{ }^{(+/ 2+)}=0.787 \mathrm{~V}$ for 21, $E_{1 / 2}{ }^{(0 /+)}=0.570$, and $E_{1 / 2}{ }^{(+/ 2+)}=0.650 \mathrm{~V}$ for 22, $E_{1 / 2}^{(0 /+)}=0.572, E_{1 / 2}^{(+/ 2+)}=0.682 \mathrm{~V}$ for 23. Coulometric titrations showed that compound 21-23 displays two sequential one electron oxidation processes, and both waves are electrochemicall fully reversible. However compound $\mathbf{2 4}$ displays three sequential oneelectron transfer steps the first two of which are merged into a single broad wave at $E_{1 / 2}^{(0 / 2+)}=$ 0.68 and third third wave appear at $E_{1 / 2}{ }^{(2+/ 3+)}=0.90 \mathrm{~V}$.
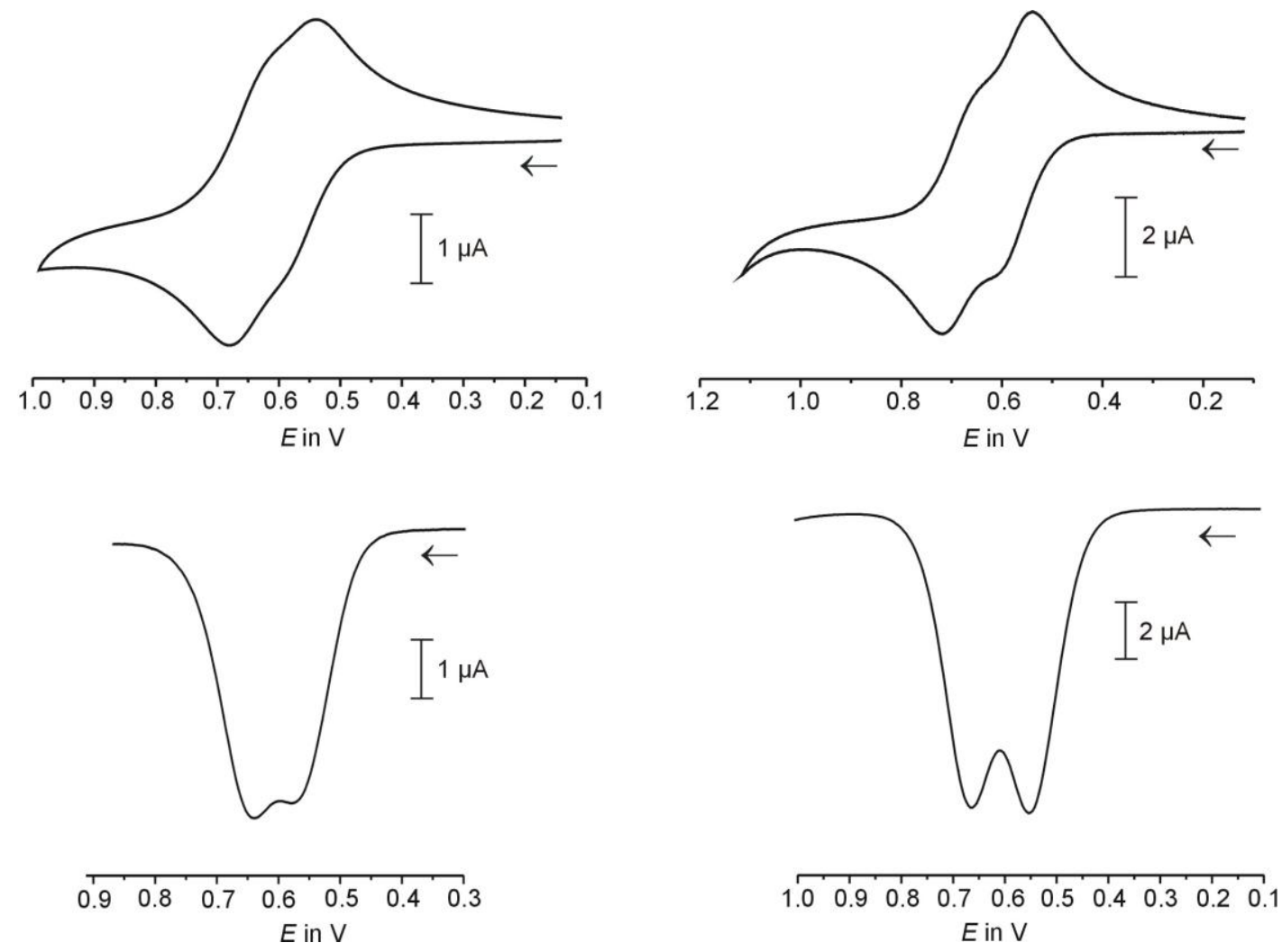

Figure 8.7. Cyclic voltammograms and square-wave voltammograms of complex 22 (in left-hand), and complex 23 (in right-hand) in dichloromethane $\left.(0.1 \mathrm{M})\left[(n-\mathrm{Bu})_{4} \mathrm{~N}\right] \mathrm{PF}_{6}\right)$ at $20^{\circ} \mathrm{C}$ recorded at a glassy carbon working electrode versus a $\mathrm{Ag} / \mathrm{AgNO}_{3}$ reference electrode. 
Both waves are electrochemically only quasireversible with a half-width of $120 \mathrm{mV}$ for the first wave and peak-to-peak separations of ca. 155 or $120 \mathrm{mV}$ for the first and the second wave respectively. In all cases no any reduction process was observed at potentials above $-1.5 \mathrm{~V}$.
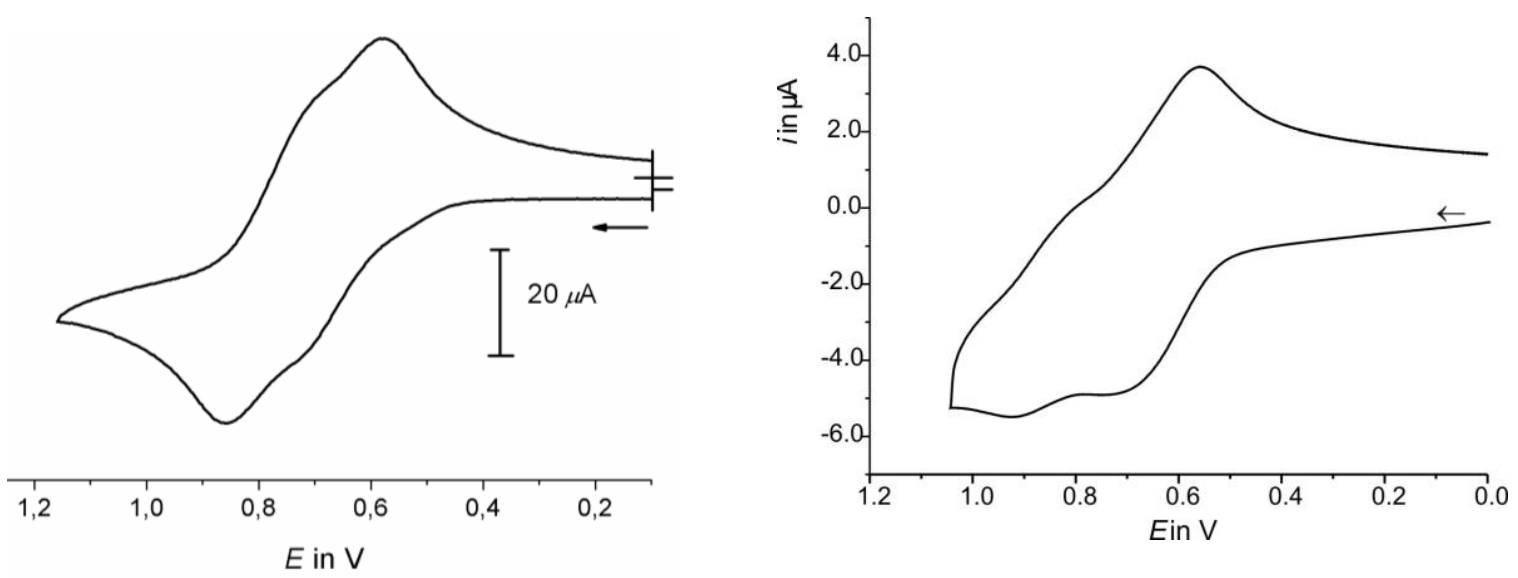

Figure 8.8. Cyclic voltammograms and square-wave voltammograms of complex 21 (left) and 24 (right) in dichloromethane $\left.(0.1 \mathrm{M})\left[(n-\mathrm{Bu})_{4} \mathrm{~N}\right] \mathrm{PF}_{6}\right)$ at $20^{\circ} \mathrm{C}$ recorded at a glassy carbon working electrode versus a $\mathrm{Ag} / \mathrm{AgNO}_{3}$ reference electrode.

As will be shown and discussed below, electrochemical oxidation of 21-24 is ligand centered and leads successively to the formation of mono and bisphenoxyl radical species for $\mathbf{2 1 - 2 3}$, and trisphenoxyl radical species for $\mathbf{2 4}$ as shown in Scheme 8.2a, and 8.2b respectively.

$\begin{aligned} & \text { (a) }\left[\mathrm{M}_{2}^{\mathrm{II}}\left(\mathrm{L}^{12}\right)(\mathrm{X})\right] \underset{+\mathrm{e}^{-}}{\rightleftharpoons} {\left[\mathrm{M}_{2}^{\mathrm{II}}\left(\mathrm{L}^{12} \bullet\right)(\mathrm{X})\right]^{+} \underset{+\mathrm{e}^{-}}{\rightleftharpoons}\left[\mathrm{M}^{\mathrm{II}}\left(\mathrm{L}^{12} \bullet \cdot\right)(\mathrm{X})\right]^{2+} } \\ & \text { (b) }\left[\mathrm{M}_{4}^{\mathrm{II}}\left(\mathrm{L}^{12}\right)_{2}\left(\mathrm{H}_{2} \mathrm{~L}^{12}\right)_{2}\right] \underset{+2 \mathrm{e}^{-}}{\stackrel{2 \mathrm{e}^{-}}{\rightleftharpoons}}\left[\mathrm{M}_{2}^{\mathrm{II}}{ }_{2}\left(\mathrm{~L}^{12} \bullet\right)_{2}\left(\mathrm{H}_{2} \mathrm{~L}^{12}\right)_{2}\right]^{2+} \underset{+\mathrm{e}^{-}}{\rightleftharpoons}\left[\mathrm{e}^{\mathrm{II}}{ }_{2}\left(\mathrm{~L}^{12} \bullet \bullet\right)_{2}\left(\mathrm{H}_{2} \mathrm{~L}^{12}\right)_{2}\right]^{3+}\end{aligned}$

Scheme 8.2: General redox properties scheme of $\left[\mathrm{M}_{2}{ }_{2}\left(\mathrm{~L}^{12}\right)(\mathrm{X})\right]\left(\mathrm{M}=\mathrm{Cu}^{2+}, \mathrm{Ni}^{2+} ; \mathrm{X}=\mathrm{OCOCH}_{3}, \mathrm{MeOH} \cdots \mathrm{OH}\right)$ 2123 (a) and $\left[\mathrm{M}_{4}^{\mathrm{II}}\left(\mathrm{L}^{12}\right)_{2}\left(\mathrm{H}_{2} \mathrm{~L}^{12}\right)_{2}\right]\left(\mathrm{M}=\mathrm{Zn}^{2+}\right) \mathbf{2 4}$ (b).

The difference between the half-wave potentials for both processes in compounds 21-24 indicates that oxidation of one phenolate moiety in the complex influences the second one, a result suggests a possible structural rearrangement of the coordination sphere upon oxidation 
and the existence of electrochemical communication through pyrazole bridged in these complexes with two equivalent phenolate groups.

Table 8.2. Redox properties of compound 21-24

\begin{tabular}{|l|l|l|l|l|l|l|}
\hline & \multicolumn{4}{|c|}{ CV data $^{[\mathrm{a}]}$} & \multicolumn{2}{c|}{ Square wave voltammetry ${ }^{[\mathrm{a}]}$} \\
\hline compound & $\begin{array}{c}\Delta E_{\mathrm{p}} \\
{[\mathrm{mV}]}\end{array}$ & $\begin{array}{c}E_{1 / 2}^{(0 /+)} \\
{[\mathrm{V}]}\end{array}$ & $\begin{array}{c}E_{1 / 2}^{(+/ 2+)} \\
{[\mathrm{V}]}\end{array}$ & $\begin{array}{c}E_{1 / 2}^{(2+/ 3+)} \\
{[\mathrm{V}]}\end{array}$ & $\begin{array}{c}E_{1 / 2}{ }^{(0 /+)} \\
{[\mathrm{V}]}\end{array}$ & $\begin{array}{c}E_{1 / 2}^{(+/ 2+)} \\
{[\mathrm{V}]}\end{array}$ \\
\hline $\mathbf{2 1}$ & 139 & 0.648 & 0.787 & & & \\
\hline $\mathbf{2 2}$ & 80 & 0.570 & 0.650 & & 0.562 & 0.654 \\
\hline $\mathbf{2 3}$ & 110 & 0.572 & 0.682 & & 0.570 & 0.685 \\
\hline $\mathbf{2 4}$ & \multicolumn{7}{|c|}{$0.680^{[\mathrm{b}]}$} & $0.900^{[\mathrm{c}]}$ & & \\
\hline
\end{tabular}

[a] Potential values given relative to the $\mathrm{Fc} / \mathrm{Fc}^{+}$reference electrode. One electron process $T=298 \mathrm{~K}$. [b] Potential corresponding to the quasireversible two-electron redox process. [c] Potential corresponding to the quasireversible one electron redox process.

\subsubsection{Spectroeletrochemistry}

8.5.3.1. Zinc(II) Complex 24: UV/Vis/NIR spectroelectrochemical measurements were recorded in $\mathrm{CH}_{2} \mathrm{Cl}_{2}$ in the range between 200 and $1000 \mathrm{~nm}$ for complex 24 (in Figure 8.9; left). The high energy band at 225, 268, and $395 \mathrm{~nm}$ is mainly associated with intraligand $\pi$ $\pi^{*}$ transition. During increase of the voltage, a new band comes at 230, 290, and $372 \mathrm{~nm}$, and some intensity at lower energy in the 425 to 450 region present at intermediate stages of the electrolysis might be due to the partially oxidized monocation which is linked to the neutral and the dictation via the comproportionation equilibrium.
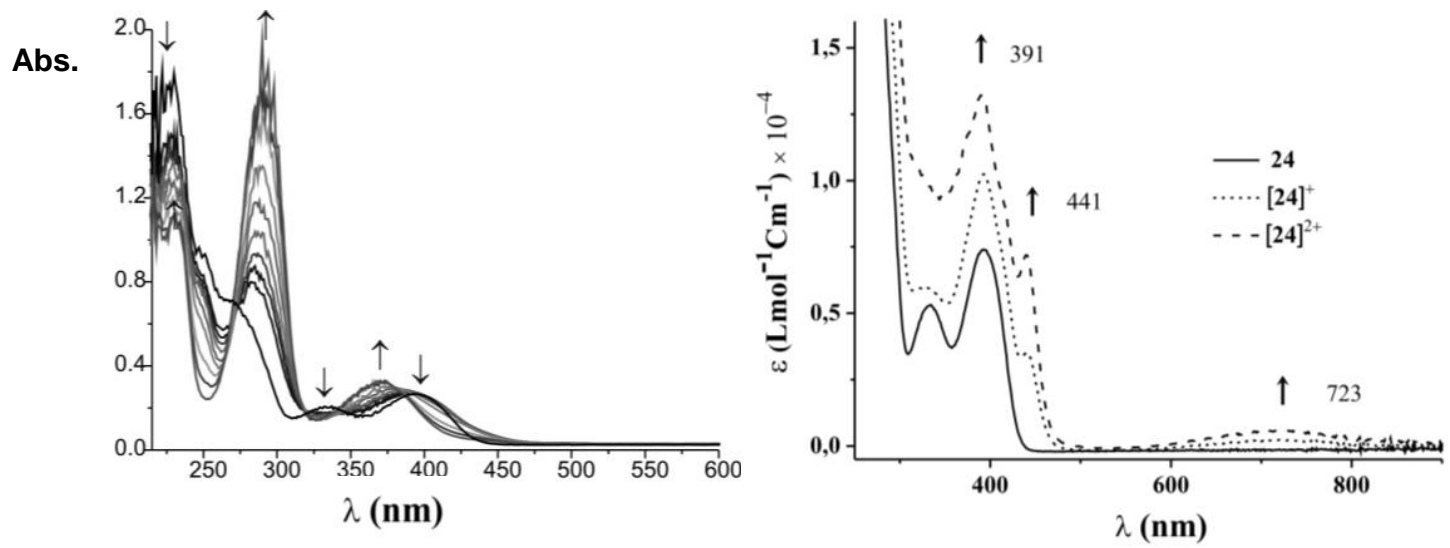

Figure 8.9. Spectroscopic change upon the stepwise oxidation of 24 in $\mathrm{CH}_{2} \mathrm{Cl}_{2} / n-\mathrm{Bu}_{4} \mathrm{PF}_{6}$ at room temperature (left), and chemical oxidation of $\mathbf{2 4}$ to $24^{2+}$ by ceric ammonium nitrare in $\mathrm{CH}_{2} \mathrm{Cl}_{2}-\mathrm{CH}_{3} \mathrm{CN}$ mixture at $-80^{\circ} \mathrm{C}$ (right). 
Further oxidation by increasing the potential to that of the third wave did not result in any noticeable changes, probably due to electrode passivation, and it might be for the same reason; partial reduction of the oxidized complex could only be achieved at large over potential.

Upon chemical oxidation of 24 in $\mathrm{CH}_{2} \mathrm{Cl}_{2}$ by $\left(\mathrm{NH}_{4}\right)_{2} \mathrm{Ce}\left(\mathrm{NO}_{3}\right)_{6}$ in actonitrile at lowtemperature caused a colour change from light yellow to green. The absorption spectrum of oxidized 24 (Figure 8.9; right) showed a characteristic band at $441 \mathrm{~nm}$, which is assigned to a $\pi \rightarrow \pi^{*}$ transition of the phenoxyl radical ligand, ${ }^{105 a}$ and a new peak at $723 \mathrm{~nm}$ is considered to arise from transitions of the phenoxyl radical ligand. ${ }^{105 b}$

8.5.3.2. Cu(II) Complexes 21 and 22: UV/Vis/NIR spectroelectrochemical measurements were recorded in $\mathrm{C}_{2} \mathrm{H}_{4} \mathrm{Cl}_{2}$ in the range between 300 and $2000 \mathrm{~nm}$ for both complexes. The electronic spectra recorded during the electrolysis are depicted in Figure 8.10 (top). The spectra of both copper (II) complex showed high energy band at $390 \mathrm{~nm}$ for phneolate-tocopper charge-transfer (CT) transition and a weak intense band at $474(\mathrm{sh}) \mathrm{nm}$, and $610 \mathrm{~nm}$ (for 21), and $605 \mathrm{~nm}$ (for 22) comes for d-d transition. During increase of the voltage, the spectra exhibited a band intensity increases at $610 \mathrm{~nm}$, and $835 \mathrm{~nm}$ and a new band comes at $440 \mathrm{~nm}$. The band at $440 \mathrm{~nm}$ corresponds to the typical transition of phenoxyl radicals expected to be found. ${ }^{36}$ The same spectral features at 441, $607 \mathrm{~nm}$ (for 21), and $438 \mathrm{~nm}$ and $603 \mathrm{~nm}$ (for 22) (Figure 8.10, bottom) were observed during chemical oxidation upon addition of 2 equivalents of ceric ammonium nitrate to the $\mathrm{CH}_{2} \mathrm{Cl}_{2}$ solution of compound 21 and 22 at $-80^{\circ} \mathrm{C}$. Thus it clearly indicates that electrochemical and chemical oxidation gave the same oxidised species in solution. 

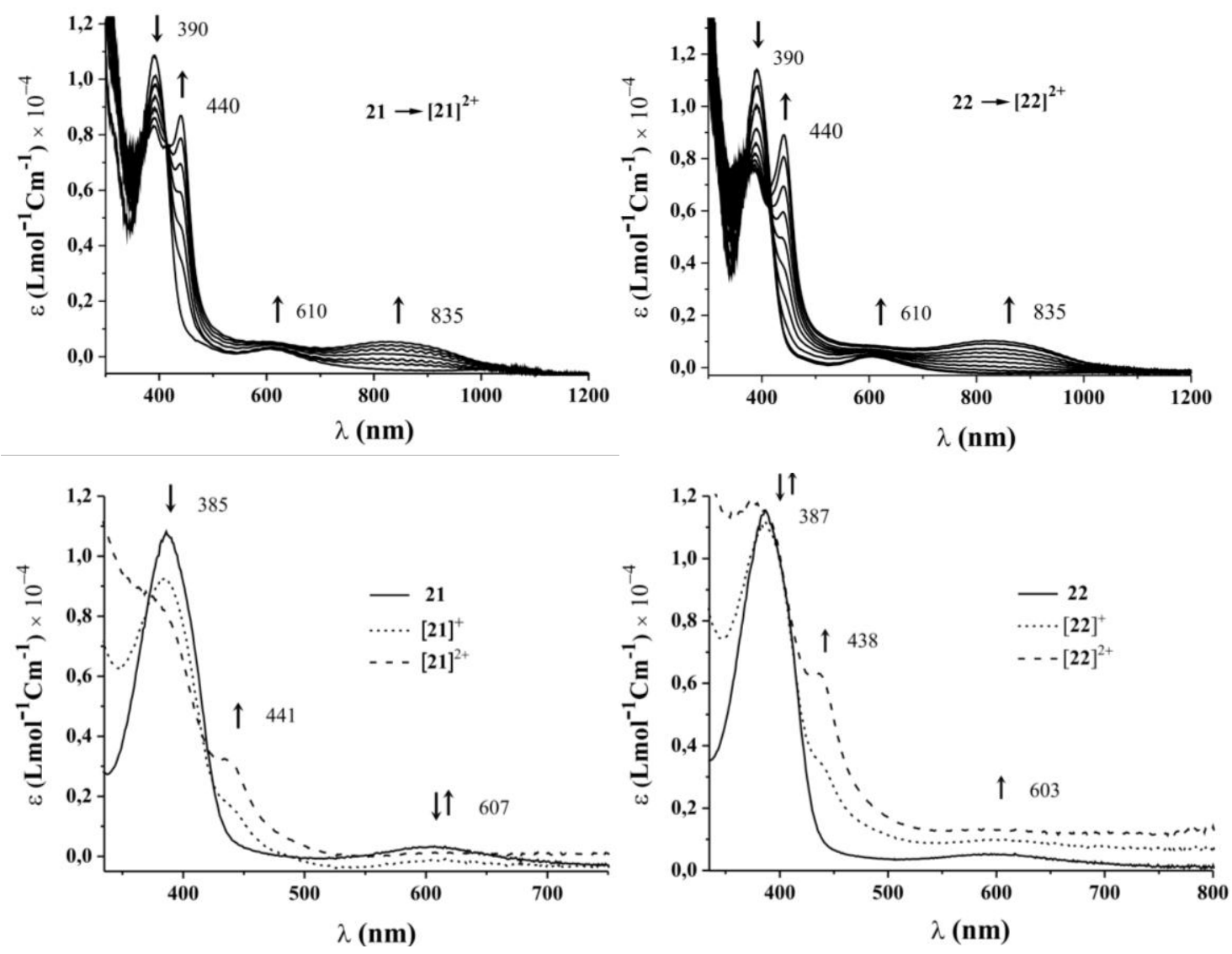

Figure 8.10. Top: Spectroscopic changes upon stepwise oxidation of $\mathbf{2 1}$ to $2 \mathbf{1}^{2+}$ (left), $\mathbf{2 2}$ to $\mathbf{2 2}^{2+}$ (right), in $\mathrm{C}_{2} \mathrm{H}_{4} \mathrm{Cl}_{2} / n-\mathrm{Bu}_{4} \mathrm{PF}_{6}$ at room temperature. Bottom: chemical oxidation of $\mathbf{2 1}$ (left), 22 (right) by ceric ammonium nitrare in $\mathrm{CH}_{2} \mathrm{Cl}_{2}-\mathrm{CH}_{3} \mathrm{CN}$ mixture at $--80^{\circ} \mathrm{C}$.

Table 8.3. Electronic Properties of compounds 21-24

\begin{tabular}{|l|l|}
\hline Compound & $\lambda_{\max }[\mathrm{nm}]\left(\varepsilon\left[10^{4} \mathrm{M}^{-1} \mathrm{~cm}^{-1}\right]\right)^{[a]}$ \\
\hline $\mathbf{2 1}$ & $390(1.09), 474 \mathrm{sh}(0.0509), 610(0.031)$ \\
\hline$[\mathbf{2 1}]^{2+}$ & $390(0.83), 440(0.871), 610 \mathrm{sh}(0.0558), 835(0.0581)$ \\
\hline $\mathbf{2 2}$ & $390(1.151), 474 \mathrm{sh}(0.0353), 605(0.0405)$ \\
\hline$[\mathbf{2 2}]^{2+}$ & $385(0.756), 440(0.8947), 610 \mathrm{sh}(0.0893), 835(0.1046)$ \\
\hline $\mathbf{2 3}$ & $328(0.950), 347(0.903), 426(0.871), 490 \mathrm{sh}(0.121)$ \\
\hline$[\mathbf{2 3}]^{2+}$ & $415(2.23), 485 \mathrm{sh}(0.6467), 910 \mathrm{sh}(0.625), 1030(0.789), 1935(0.437)$ \\
\hline$\left[\mathbf{2 3}-\mathrm{py}_{4}\right]^{2+[b]}$ & $403(1.87), 485 \mathrm{sh}(0.459)$ \\
\hline $\mathbf{2 4}]^{[b]}$ & $332(0.532), 393(0.744)$ \\
\hline$[\mathbf{2 4}]^{2+[b]}$ & $320 \mathrm{sh}(1.061), 391(1.325), 441(0.725), 723(0.0598)$ \\
\hline
\end{tabular}

[a] UV/Vis spectra recorded in $\mathrm{C}_{2} \mathrm{H}_{4} \mathrm{Cl}_{2}-1,2 / n-\mathrm{Bu}_{4} \mathrm{PF}_{6}(0.1 \mathrm{M})$ at $298 \mathrm{~K}$; sh = shoulder, br = broad [b] Spectrum recorded by chemical oxidation with ceric ammonium nitrate in $\mathrm{CH}_{2} \mathrm{Cl}_{2}-\mathrm{CH}_{3} \mathrm{CN}$ mixture at $-80^{\circ} \mathrm{C}$. 
8.5.3.3. Ni(II) Complex 23: UV/Vis/NIR spectroelectrochemical measurements were recorded in $\mathrm{C}_{2} \mathrm{H}_{4} \mathrm{Cl}_{2}$ in the range between 300 and $2400 \mathrm{~nm}$ for complex 23 in Figure 8.11. The spectrum of neutral nickel (II) complex $\mathbf{2 3}$ is dominated by intense absorptions at 328 , 347, 426, and a weak shoulder at $490 \mathrm{~nm}$. The $426 \mathrm{~nm}$ corresponds to the charge-transfer (CT) transition. ${ }^{99 \mathrm{c}}$ The shorter wavelength features are associated with $\pi-\pi^{*}$ transitions, while the longer wave legth bands are correspond to the $\mathrm{d}$ - $\mathrm{d}$ transitions of $\mathrm{a} \mathrm{d}^{8}$ low spin $\mathrm{Ni}^{\mathrm{II}}$ center in a square palanr geometry. During increase of the voltage, a shoulder comes at $485 \mathrm{~nm}$ and the band is shifted from 426 to $415 \mathrm{~nm}$ which is corresponds to the typical transition of phenoxyl radicals are expected to be found. ${ }^{99}$ In addition two new peak comes in the near-infrared (NIR) region at 1030, and $1935 \mathrm{~nm}$, such kind of low energy and high intensity absorptions features are the best assignments to phenolate-phenoxyl intramolecular charge transfer transition (IVCT). ${ }^{99 \mathrm{e}}$ These data thus suggests a significant ligand radical character for $\mathbf{2 3}^{2+}$. Upon chemical oxidation of 23 in $\mathrm{CH}_{2} \mathrm{Cl}_{2}$ by $\left(\mathrm{NH}_{4}\right)_{2} \mathrm{Ce}\left(\mathrm{NO}_{3}\right)_{6}$ in actonitrile at lowtemperature caused a colour change from yellow to brown. The absorption spectrum of oxidized 23 (Figure 8.12; left) exhibited characteristic new peak at $795 \mathrm{~nm}$, which corresponds to CT transition, and a band shifted from 423 to $411 \mathrm{~nm}$ indicates phenoxyl radicals, which are expected to be found in the electrochemical oxidation of the oxidised species. ${ }^{99}$ Interestingly, upon addition of pyridine to the oxidised species of $\mathbf{2 3}^{2+}$ at $-80^{\circ} \mathrm{C}$ caused a drastic colour change from brown to green. Hence the binding tendency of $\mathbf{2 3}^{2+}$ to pyridine was obtained from $\mathrm{UV} / \mathrm{V}$ is titration in $\mathrm{CH}_{2} \mathrm{Cl}_{2}-\mathrm{CH}_{3} \mathrm{CN}$ mixture (Figure 8.12; right).

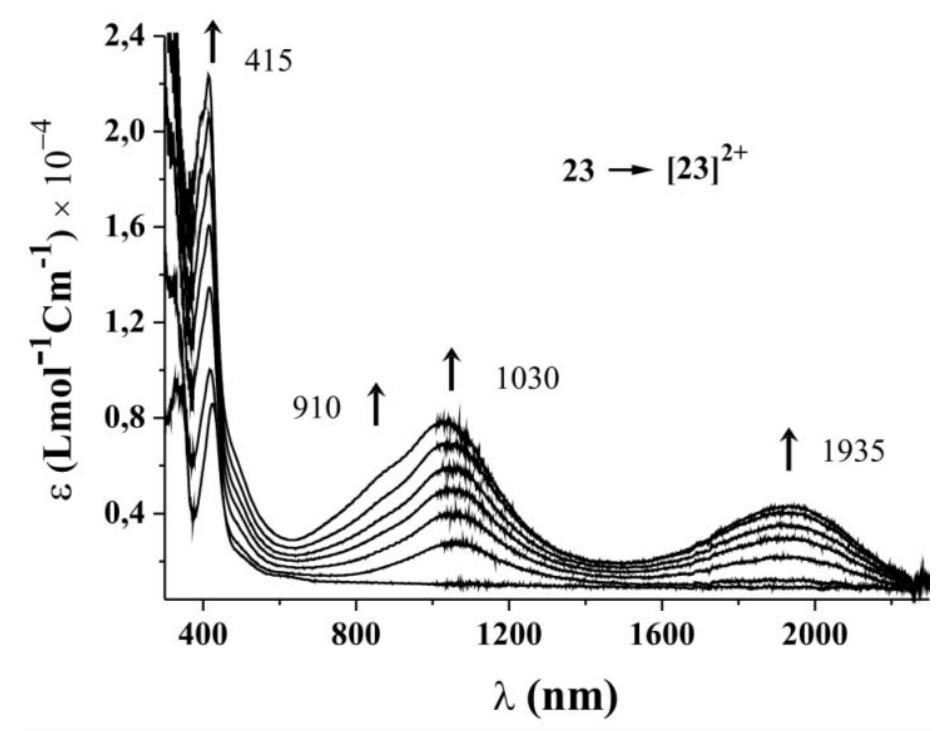

Figure 8.11. Spectroscopic changes upon the stepwise oxidation of 23 to $\mathbf{2 3}^{2+}$ in $\mathrm{C}_{2} \mathrm{H}_{4} \mathrm{Cl}_{2} / n-\mathrm{Bu}_{4} \mathrm{PF}_{6}$ at room temperature. 

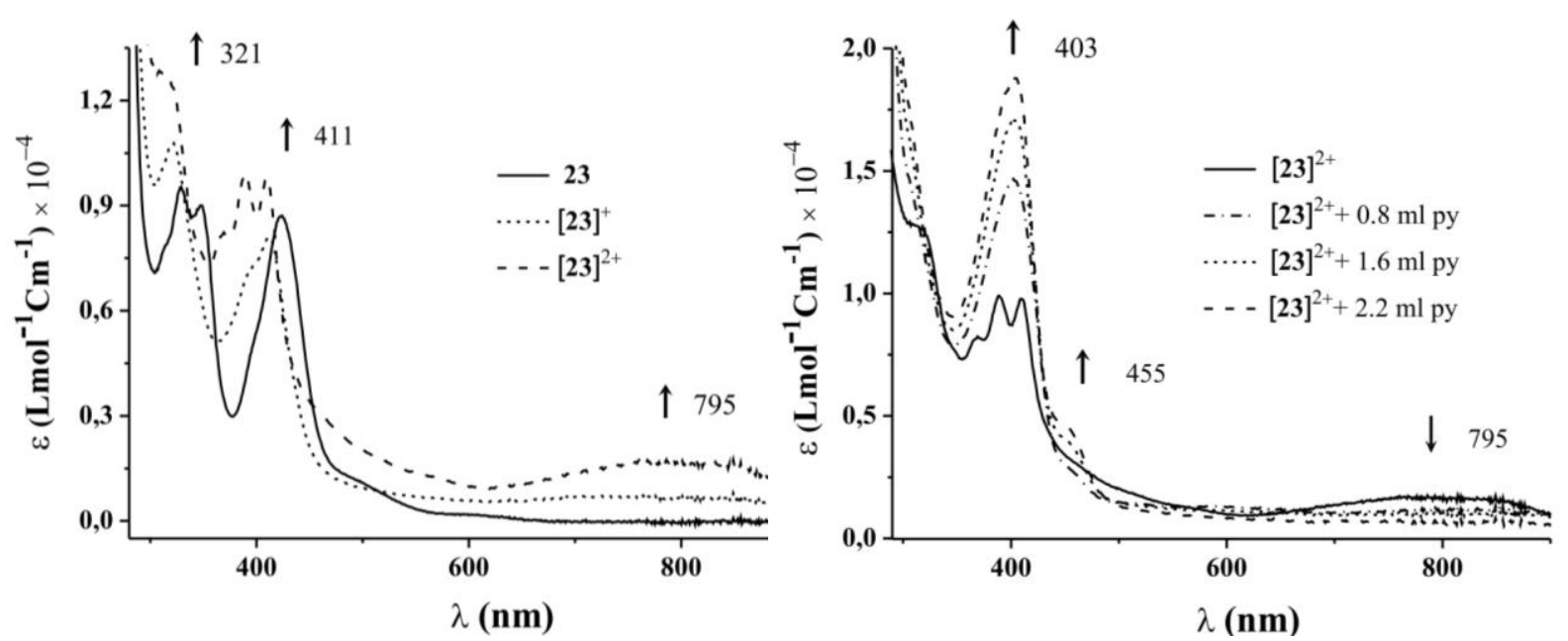

Figure 8.12. Spectroscopic changes upon chemical oxidation of $\mathbf{2 3}$ to $\mathbf{2 3}^{2+}$ by ceric ammonium nitrate (left) in $\mathrm{CH}_{2} \mathrm{Cl}_{2}-\mathrm{CH}_{3} \mathrm{CN}$ mixture at $-80^{\circ} \mathrm{C}$ and addition of pyridine to the oxidised species (right) in $\mathrm{CH}_{2} \mathrm{Cl}_{2}-\mathrm{CH}_{3} \mathrm{CN}$ mixture at $-80^{\circ} \mathrm{C}$.

The absorption spectrum of the oxidised species of $\mathbf{2 3}^{2+}$ with pyridine exhibits a new shoulder at $455 \mathrm{~nm}$, and the band intensity drastically increased at $403 \mathrm{~nm}$. And a high intense CT band at $795 \mathrm{~nm}$ is almost disappearing. Such kinds of absorption features are nicely fitted with $\mathrm{Ni}^{\mathrm{III}}$ species in soluion. ${ }^{99}$ Thus it predicts that weak donor pyridine molecule is axially bounded to the metal ions formed an octahedral geometry and stabilised the high oxidation $\mathrm{Ni}^{\mathrm{III}}$ species.

\subsubsection{EPR-Spectroscopy}

8.5.4.1. Zinc(II) Complex 24: X-band EPR spectra of $[24]^{2+}$ (generated chemically by the reaction of 24 with 2 equivalent ceric ammonium nitrate) in $\mathrm{CH}_{2} \mathrm{Cl}_{2}-\mathrm{CH}_{3} \mathrm{CN}$ mixture have been recorded at $147 \mathrm{~K}$. The spectrum is depicted in Figure 8.13. It exhibit typical isotropic signal at $g=2.006$, which is characteristic for phenoxyl radical ${ }^{105 a}$ by ligand centered oxidation. There is no any additional coupling observed between the two radicals. 


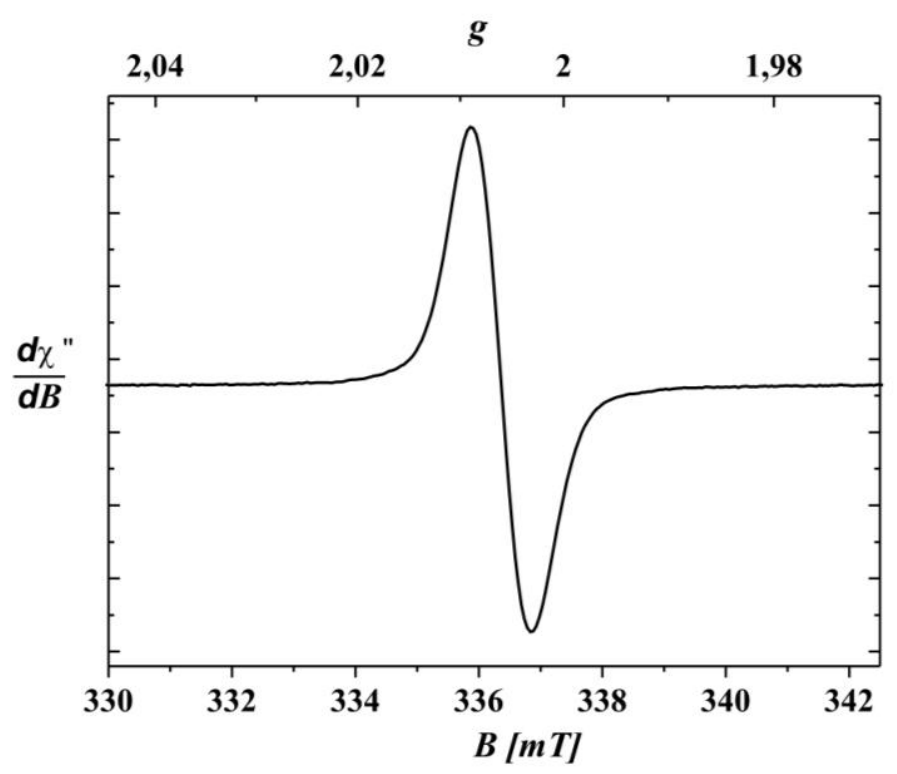

Figure 8.13. $\mathrm{X}$-band EPR spectrum of $[\mathbf{2 4}]^{2+}$ in $\mathrm{CH}_{2} \mathrm{Cl}_{2}-\mathrm{CH}_{3} \mathrm{CH}_{2} \mathrm{CN}$ mixture $(1 \mathrm{mM})$ at $147 \mathrm{~K}$.

8.5.4.2. Copper(II) Complex 21-22: Since the redox potential of the $E_{1 / 2}{ }^{0 / 2+}$ (Table 8.2) for the $\mathrm{Zn}^{\mathrm{II}}$ and $\mathrm{Cu}^{\mathrm{II}}$ complexes are similar, thus it may be anticipated that the redox chemistry of both $\mathrm{Cu}^{\mathrm{II}}$ complexes are also ligand-based oxidation and giving rise to bisphenoxyl radicals. The X-band EPR spectra of compound 21, and 22 in $\mathrm{CH}_{2} \mathrm{Cl}_{2}$ were recorded at $160 \mathrm{~K}$. It display a broad signal at $g=2.09$ which is characteristic for exchanged couple $\mathrm{Cu}^{\mathrm{II}}$ complex. The EPR spectra of compound $[\mathbf{2 1}]^{2+}$ and $[\mathbf{2 2}]^{2+}$ (generated chemically by the reaction of 21 and 22 with 2 equivalent ceric ammonium nitrate) in $\mathrm{CH}_{2} \mathrm{Cl}_{2}-\mathrm{CH}_{3} \mathrm{CH}_{2} \mathrm{CN}$ mixture were recorded at $170 \mathrm{~K}$. The spectra are showed in Figure 8.14. In both cases oxidised species leads to attenuation of their EPR signals to $<15 \%$ of their original intensity $(g=2.09 ; 170 \mathrm{~K})$, consistent with the formation of phenoxyl radical is antiferromagnetically coupled to $\mathrm{Cu}^{\text {II }}$ ion. ${ }^{36 \mathrm{a}}$ Thus from the EPR spectroscopy it is evident that the oxidation of $\mathrm{Cu}^{\mathrm{II}}$ is ligand based. 

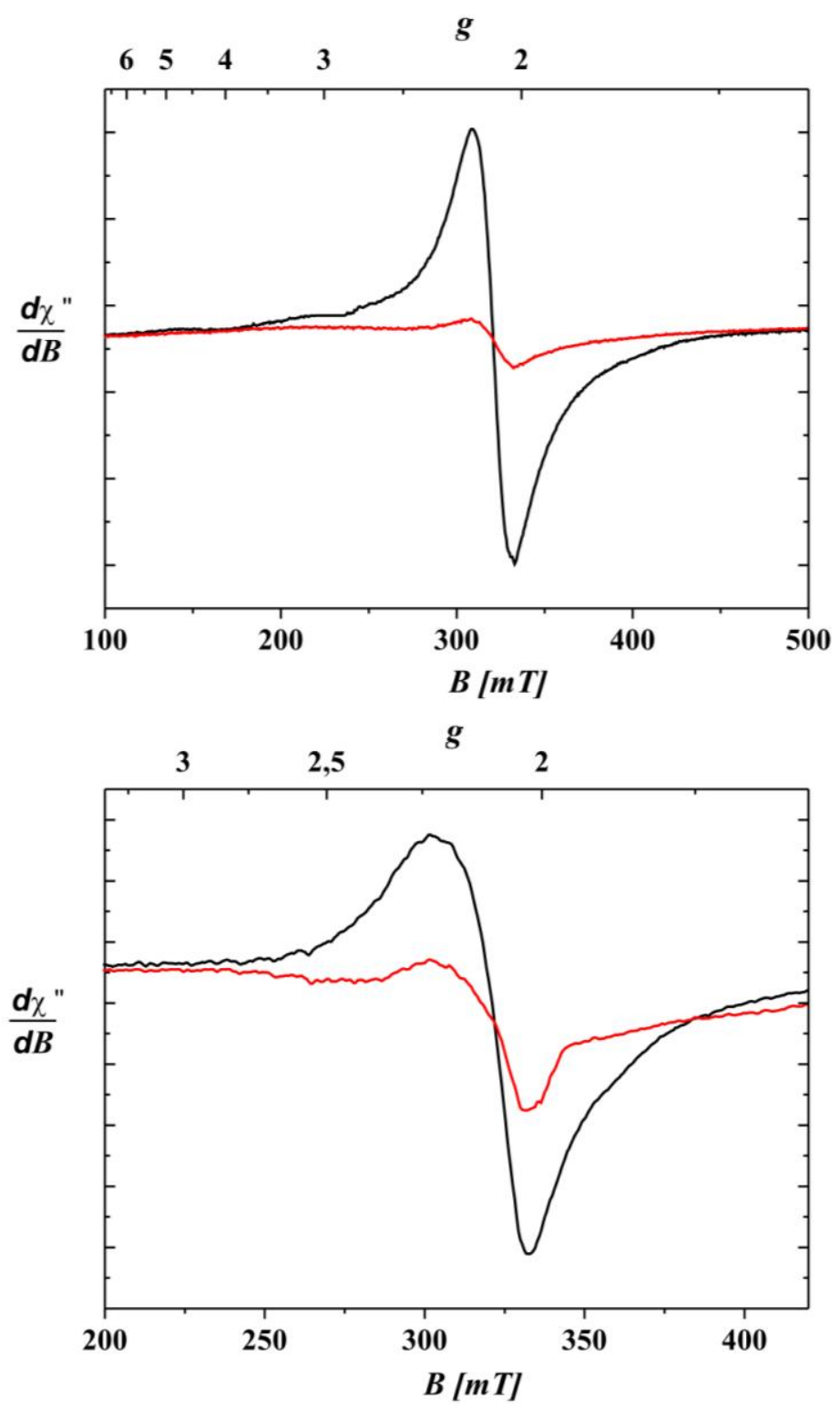

Figure 8.14. (Top): X-band EPR spectrum of 21 (black line), $[\mathbf{2 1}]^{2+}$ (red line) and (bottom): 22 (black line), $[22]^{2+}$ (red line) in $\mathrm{CH}_{2} \mathrm{Cl}_{2}-\mathrm{CH}_{3} \mathrm{CH}_{2} \mathrm{CN}$ mixture $(1 \mathrm{mM})$ at $170 \mathrm{~K}$.

8.5.4.2. Nickel(II) Complex 23: The X-band EPR spectrum of $[23]^{2+}$ (generated chemically by the reaction of 23 in $\mathrm{CH}_{2} \mathrm{Cl}_{2}$ with 2 equivalent ceric ammonium nitrate in $\mathrm{CH}_{3} \mathrm{CH}_{2} \mathrm{CN}$ ) have been recorded at $160 \mathrm{~K}$. The spectrum is depicted in Figure 8.15. It showed an unresolved signal at $g_{\text {iso }}=2.006$, which is characteristic for coordinated phenoxyl radicals ${ }^{99}$ by ligand centered oxidation. In contrast X-band EPR spectrum of [23-py $]^{2+}$ in $\mathrm{CH}_{2} \mathrm{Cl}_{2-}$ $\mathrm{CH}_{3} \mathrm{CH}_{2} \mathrm{CN}$ with coordinating ligand pyridine at $4.7 \mathrm{~K}$, displayed a rhombic signal (Figure 8.15), with a $g$ tensor anisotropy $\left(g_{z}=2.21, g_{\mathrm{y}}=2.04, g_{\mathrm{x}}=2.00\right.$, and $\left.g_{\mathrm{av}}=2.09\right)$, indicating a rhombic symmetry known for the nickel centered oxidised species. ${ }^{99}, 106$ 


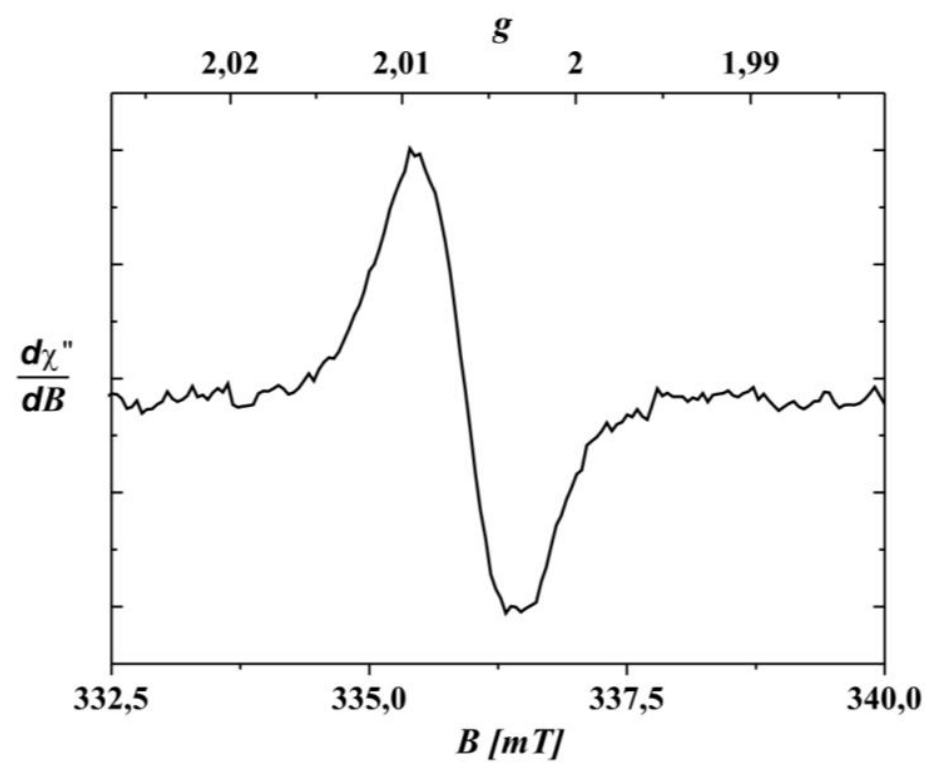

Figure 8.15. X-band EPR spectrum of $[23]^{2+}$ in $\mathrm{CH}_{2} \mathrm{Cl}_{2}-\mathrm{CH}_{3} \mathrm{CH}_{2} \mathrm{CN}$ mixture $(1 \mathrm{mM})$ at $160 \mathrm{~K}$.
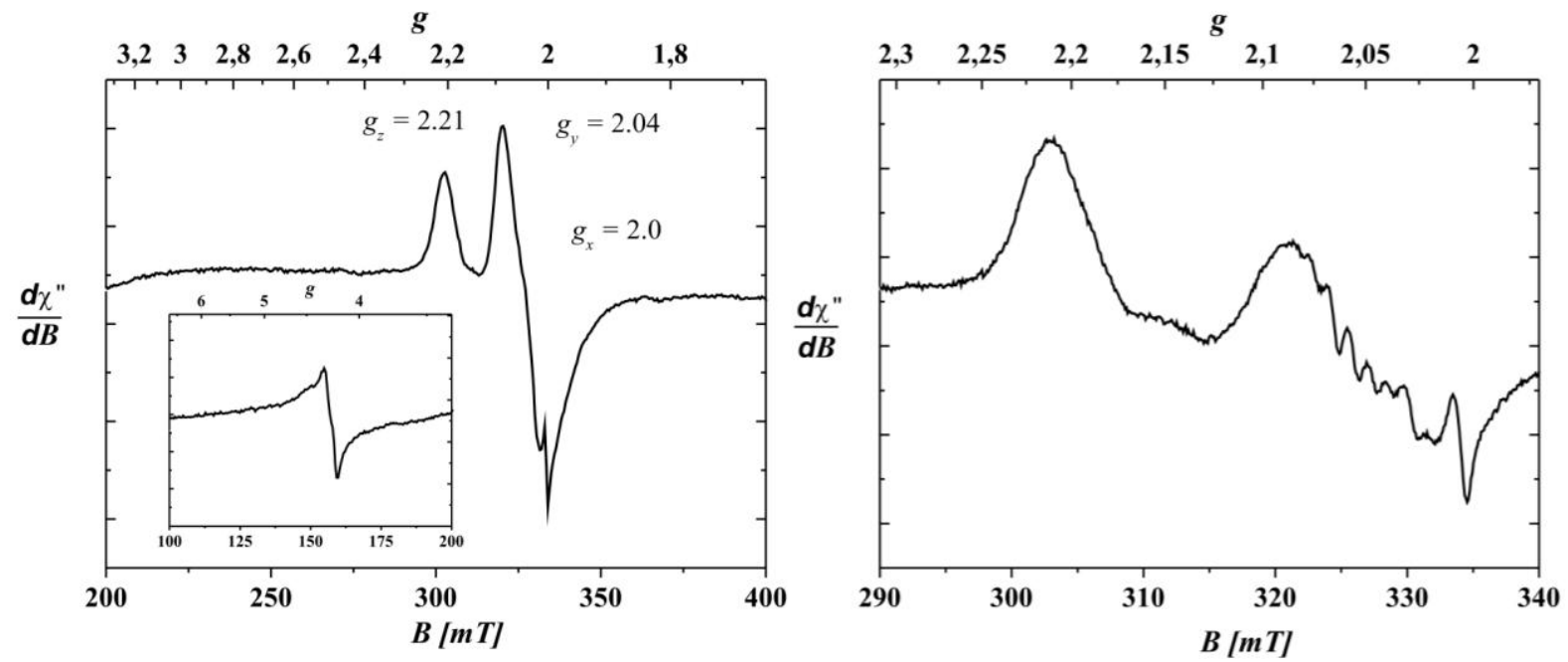

Figure 8.16. X-band EPR spectrum of $\left[23-\mathrm{py}_{4}\right]^{2+}$ (generated chemically by the reaction of $[23]^{2+}$ with excess pyridine) in $\mathrm{CH}_{2} \mathrm{Cl}_{2}-\mathrm{CH}_{3} \mathrm{CH}_{2} \mathrm{CN}$ mixture $(1 \mathrm{mM}$ ) at $4.7 \mathrm{~K}$ (left), and at $17 \mathrm{~K}$ (right). Inset displays half-filled splitting.

The $g_{\text {av }}$ value is lower than any reported for nickel(III) complexes ${ }^{106,99}$ or high spin nickel (II) magnetically coupled to radical. ${ }^{107}$ On the other hand it is higher than those of phenoxyl radical, suggesting $\left[\mathbf{2 3}-\mathrm{py}_{4}\right]^{2+}$ is ligand radical with contribution from the singly occupied $\mathrm{d}$ orbital of $\mathrm{Ni}^{\mathrm{iII}}$. The pattern of the $g$ values, $g_{\mathrm{z}}(2.21), g_{\mathrm{y}}(2.04)>g_{\mathrm{x}}(2.0) \approx g_{\mathrm{e}}(2.0)$ is indicative of a $d_{x 2-y 2}$ ground state where the $\mathrm{x}$ - and $\mathrm{y}$-axis is in the plane of the molecule, and $\mathrm{z}$-axis is the perpendicular to the molecule. In addition to that the presence of superfine 
pattern in the high field component at $17 \mathrm{~K}$ (Figure 8.16) implies that the $\mathrm{d}_{x 2-y 2}$ magnetic orbital of each low spin $\mathrm{Ni}^{\mathrm{III}}$ of $\left[\mathbf{2 3}-\mathrm{py}_{4}\right]^{2+}$ couples with both equatorially bonded imine $\mathrm{N}$, and pyrazole N. But probably due to the superimposition, instead of ten-line hyperfine spectrum an eight-line hyperfine spectrum is observed. To gets the idea about site of the oxidation of $\mathrm{Cu}^{\mathrm{II}}$ and $\mathrm{Ni}^{\mathrm{II}}$ complex, DFT calculation is also needed that is under progress.

\subsection{Conclusions}

In this chapter we have described the synthesis of novel phenol-pyrazole based compartmental ligand $\left(\mathbf{H}_{\mathbf{3}} \mathbf{L}^{\mathbf{1 2}}\right)$ which coordinates to the different transition metal ions to form a series of complexes having varied structural motif and nuclearity. It is particularly interesting to find that Schiff base type ligand can be redox noninocent. It is possible to oxidise these complexes electrochemically. Complexes 21-23 possesess two reversible one electron oxidations in the redox process, while compound $\mathbf{2 4}$ exhibit three quasireversible one-electron oxidations in the cyclic voltammogram. In all cases, two phenol side arms of the ligand is oxidised, and relatively stable bis-phenoxyl radical species formed, those were cahracterised thoroughly by UV-visible spectroscopy (electrochemically and chemically) and EPR spectroscopy (Scheme 8.3a, and 8.3b).

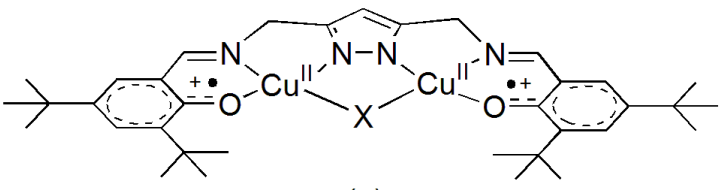

(a)

Antiferromagnetically coupling between the radical and copper(II)

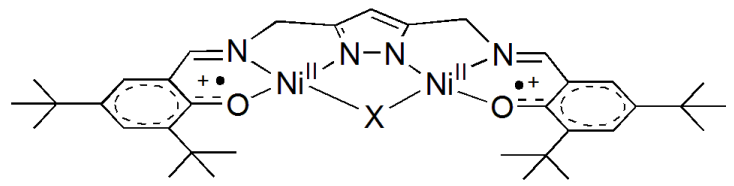

(b)

Delocalised radicals

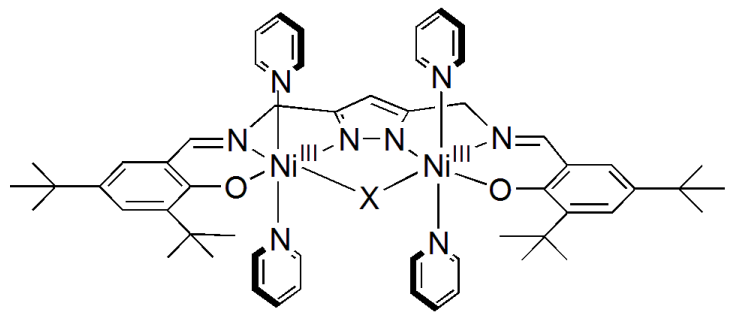

(c)

Phenolate-nickel(III)

Scheme 8.3. Modulation of the oxidation locus by ligand field and coordination of pyridine. (a) $\mathrm{X}=\mathrm{MeOH} \cdot \mathrm{OH}$, $\mathrm{OCOCH}_{3}$ (b) $\mathrm{X}=\mathrm{OCOCH}_{3}$ (c) $\mathrm{X}=\mathrm{OCOCH}_{3}$.

Interestingly here we found that oxidised species of $\mathbf{2 3}$ yielded corresponding six coordinated low-spin $\mathrm{Ni}(\mathrm{III})$ species in presence of pyridine. Thus locus of the oxidation of $\mathrm{Ni}^{\mathrm{II}}$ complex 
has been changed from $\mathrm{Ni}$ (II)-phenoxyl radical to $\mathrm{Ni}(\mathrm{III})$-phenoxide so called valence tautomerism occurs in presence of exogenous $\mathrm{N}$-donor ligand pyridine at low temperature (Scheme 8.3c). 


\section{Chapter 9}

\section{Experimental Section}

\subsection{General Considerations}

All manipulations were carried out under an anaerobic and anhydrous atmosphere of dry dinitrogen by employing standard Schlenk techniques or in a glovebox, unless mentioned otherwise. $\mathrm{Et}_{2} \mathrm{O}$ and pentane were dried over sodium benzophenone ketyl; THF, benzene, toluene and hexanes over potassium benzophenone ketyl; DMF, DMSO, $\mathrm{MeCN}, \mathrm{CH}_{2} \mathrm{Cl}_{2}$, and $\mathrm{CHCl}_{3}$ over $\mathrm{CaH}_{2} ; \mathrm{MeOH}$ and $\mathrm{EtOH}$ over $\mathrm{Mg}$, and distilled prior to use. Compounds 2bromo-3-(trimethylsilyloxy)pyridine, ${ }^{38}$ pyridine-2-carboxylic acid hydrazide, ${ }^{37}$ 1-(2hydroxyphenyl)-3-dimethylaminoprop-2-enone, ${ }^{59}$ 1-(2-hydroxyphenyl)-3-phenylpropane-1,3dione, ${ }^{60} 3$-(2-methoxyphenyl)pyrazole ${ }^{60}$ and 3,5-bis(aminomethyl) pyrazole dihydrochloride, ${ }^{103}$ were synthesized by previously reported procedures. All other chemicals were purchased from commercial sources and used as received. Microanalyses were performed by the Analytical Laboratory of the Institute for Inorganic Chemistry at Georg-August-University, Göttingen. IR spectra (as KBr pellets) were recorded with a Digilab Excalibur, and UV-vis spectra of solutions and solids (diffuse reflectance) with a Varian Cary 5000 spectrometer at room temperature and low temperature. Mass spectra were measured using a Finnigan MAT 8200 (EI), a Finnigan MAT 95 (FAB-MS), or a Finnigan MAT LCQ mass spectrometer (ESIMS). ${ }^{1} \mathrm{H}$ NMR and ${ }^{13} \mathrm{C}$ NMR spectra were recorded on a Bruker Avance $200 \mathrm{MHz}, 300 \mathrm{MHz}$ or $500 \mathrm{MHz}$ spectrometer and calibrated to the residual proton and carbon signals of the solvent $\left(\mathrm{CDCl}_{3} ; \delta \mathrm{H} 7.26\right.$ ppm, $\delta \mathrm{C} 77.0$ ppm, and DMSO- $d_{6} ; \delta \mathrm{H} 2.49$ ppm, $\delta \mathrm{C} 29.9$ ppm). Xband EPR derivative spectra were recorded on a Bruker ELEXSYS E500 spectrometer equipped with the Bruker standard cavity (ER4102ST) and a helium flow cryostat (Oxford Instruments ESR 910). Microwave frequencies were calibrated with a Hewlett-Packard frequency counter (HP5352B), and the field control was calibrated with a Bruker NMR field probe $(\mathrm{ER} 035 \mathrm{M})$. The spectra were simulated with the program GFIT for the calculation of powder spectra with effective g values and anisotropic line widths (Gaussian line shapes were used). Variable temperature, solid-state direct current (dc) and alternating current (ac) magnetic susceptibility data down to $1.8 \mathrm{~K}$ were collected on a Quantum Design MPMS-5S SQUID magnetometer equipped with a $5 \mathrm{~T}$ dc magnet (for these measurements the powdered samples were contained in a gel bucket and fixed in a non-magnetic sample holder). Diamagnetic corrections were applied to the observed paramagnetic susceptibilities using Pascal's constants. Magnetic studies below $1.8 \mathrm{~K}$ were carried out on single crystals using a 
magnetometer consisting of a micro-Hall bar. High-field/high-frequency electron spin resonance (HF-ESR) studies in a frequency range $50-400 \mathrm{GHz}$ in magnetic fields up to $15 \mathrm{~T}$ were performed using a home made spectrometer based on a Millimeterwave Vector Network Analyzer (AB Millimetré).

\subsection{Synthesis of Ligands and Ligand Precursors}

2,4,6-Tris( $N$-hydrazino-pyridyl-2-carboxylate)-1, 3, 5-triazine $\left(\mathrm{H}_{3} \mathrm{~L}^{\mathbf{1}}\right)$ : To a stirred solu tion of 2,4,6-trichloro-1,3,5-triazine (448 mg, $2.43 \mathrm{mmol}, 1$ eqv.) in $40 \mathrm{ml}$ acetonitrile, was added dropwise pyridine-2-carboxylic acid hydrazide (2 gm, $14.59 \mathrm{mmol}, 6 \mathrm{eqv}$.) in $20 \mathrm{ml}$ $\mathrm{CH}_{3} \mathrm{CN}$ at room temperature. After refluxing for $3 \mathrm{hrs}$, the resulting mixture was cooled to room temperature, and then the precipitate was filtered, washed with acetonitrile to reduce the contamination of pyridine-2-carboxylic acid hydrazide hydrochloride salt, and then dried in air. Yield: $30 \% .{ }^{1} \mathrm{H}$ NMR (200 MHz, DMSO- $d_{6}$ ): $\delta 11.01$ (br, 3H), 10.50 (br, $3 \mathrm{H}$ ), 8.73-8.65 $(\mathrm{m}, 3 \mathrm{H}), 8.29-8.22$ (br, 3H), 8.12-8.02 (m, 3H), 7.78-7.62 (m, 3H), 7.49-7.43 (br, 3H). ${ }^{13} \mathrm{C}\left\{{ }^{1} \mathrm{H}\right\}$ NMR (75.47 MHz, DMSO- $d_{6}$ ): $\delta 121.6$ (py-C), 126.10 (py-C), 137.50 (py-C), 148.38 (py-C), 149.78 (py-C), 162.50 (N-C). Mass spectra: MS (EI) m/z: 486.1 (100) [M] $]^{+}$ Elemental analysis (\%) calculated for $\mathrm{C}_{21} \mathrm{H}_{18} \mathrm{~N}_{12} \mathrm{O}_{3}$ (486.4) : C-51.85, H-3.73, N-34.55; found: C-50.89, H-3.41, N-33.30.

2,4,6-Tris(3-hydroxy-2-pyridyl)-1,3,5-triazine $\left(\mathbf{H}_{3} \mathbf{L}^{2}\right)$ : To a solution of 2-bromo-3-(trim ethyl silyloxy) pyridine (2 gm, $8.1 \mathrm{mmol}$, 1eqv.) in $35 \mathrm{ml}$ THF was added ${ }^{\mathrm{n}-} \mathrm{BuLi}(2.5 \mathrm{M}$ in hexane, $22 \mathrm{ml}, 8.5 \mathrm{mmol}, 1.05 \mathrm{eqv}$.) at $-78^{\circ} \mathrm{C}$ under argon. After 1 hour, cyanuric chloride (433 mg, $2.35 \mathrm{mmol}, 0.29 \mathrm{eqv}$.) in $10 \mathrm{ml}$ THF was added drop wise to the reaction mixture, stirred at $-78^{\circ} \mathrm{C}$ for 2 hours, and then stirred overnight at ambient temperature, after completion of the reaction, all the solvent was evaporated under reduced pressure gave a reddish yellow crude product, stored under nitrogen atmosphere, and then added dry diethyl ether to separate the inorganic salt, evaporate the ether soluble part under vacuum, and treated with dry methanol to deprotect the silyloxy group, yilded an oily reddish yellow crude product, and then washed with $n$-hexane, becomes a red solid. Finally it was purified by column chromatography under silica gel with 1:9 MeOH/ EtOAc gave a yellowish white solid. Yield. 11\%. ${ }^{1} \mathrm{H}$ NMR (500 MHz, $\mathrm{CDCl}_{3}$ ): $\delta 8.45$ (dd, 2H, OH, 6-Ar-H), 7.47 (dd, 1H, 4-Ar-H), 7.30 (dd, 1H, 5-Ar-H). ${ }^{13} \mathrm{C}\left\{{ }^{1} \mathrm{H}\right\}$ NMR (75.47 MHz, $\mathrm{CDCl}_{3}$ ): $\delta 123.99$ (py-C), 129.07 (py-C), 143.10 (py-C), 147.38 (py-C), 148.10 (py-C), 173.36 (N-C). Mass spectra: MS (EI) $m / z: 360.1$ (45) $[\mathrm{M}]^{+}$. Anal. Calcd for $\mathrm{C}_{18} \mathrm{H}_{12} \mathrm{~N}_{6} \mathrm{O}_{3}$ : C-60.00, H-3.36, N-23.32, O-13.32. Found: C-58.97, H-3.49, N-22.54. 
2,4,6-Tris( $N$-methylhydrazino)-1,3,5-triazine (1): 2,4,6-Trichloro-[1,3,5]-triazine (3 gm, $16.3 \mathrm{mmol}$, 1equiv) in $50 \mathrm{ml}$ dry acetonitrile was added drop wise to the solution of methylhydrazine $\left(5.15 \mathrm{ml}, 97.8 \mathrm{mmol}, 6\right.$ equiv) in $100 \mathrm{ml}$ acetonitrile at $0^{\circ} \mathrm{C}$, after completion of the addition, the reaction mixture was warmed to room temperature, stirring $2 \mathrm{hrs}$ at room temperature, and then refluxed for $3 \mathrm{hrs}$. The white solid hydochloride salt was precipitated on cooling and removed by filtration, and then resulting solution was concentrated in vacuum to give a white solid. Yield: $1.6 \mathrm{gm}(46 \%)$. Mp $150^{\circ} \mathrm{C}$. MS (ESI, MeOH): $\mathrm{m} / \mathrm{z}(\%)=214.2$ (100) $[\mathrm{M}+\mathrm{H}]^{+}, 199.2(25)\left[\mathrm{M}-\mathrm{CH}_{3}\right]^{+} .{ }^{1} \mathrm{H} \mathrm{NMR}\left(500 \mathrm{MHz}, \mathrm{CDCl}_{3}\right): \delta 5.00\left(\mathrm{br}, 6 \mathrm{H}, \mathrm{NH}_{2}\right), 3.15$ $\left(\mathrm{m}, 9 \mathrm{H}, \mathrm{CH}_{3}\right) \mathrm{ppm} .{ }^{13} \mathrm{C}$ NMR $\left(125.75 \mathrm{MHz}, \mathrm{CDCl}_{3}\right)$ : $\delta 164.418$ (triazine-C), $37.008\left(\mathrm{CH}_{3}\right)$. IR (KBr): ขึ 3321 (m), 3250 (br), 3150 (br), 2930 (w), 2196 (m), 1684 (s), 1624 (w), 1578 (s), 1492 (m), 1397 (m), 1369 (w), 1323 (w), 1225 (w), 1155 (w), 1070 (w), 1009 (br), 925 (m),

$841(\mathrm{~m}), 796(\mathrm{w}), 747(\mathrm{w}), 734(\mathrm{~m}), 643(\mathrm{w}) \mathrm{cm}^{-1}$. Elemental analysis (\%) calculated for $\mathrm{C}_{6} \mathrm{H}_{16} \mathrm{~N}_{9} \mathrm{Cl}$ (249.7) : C-28.86, H-6.46, N-50.48; found: C-28.89, H-6.41, N-50.04.

2,4,6-Tris $\{N$-(2-hydroxyethylhydrazino)\}-1,3,5-triazine (2): 2,4,6-Trichloro-[1,3,5]-triazine ( $3 \mathrm{gm}, 16.3 \mathrm{mmol}, 1$ equiv) in $50 \mathrm{ml}$ dry acetonitrile was added drop wise to the solution of 2 hydroxyethylhydrazine $\left(4.98 \mathrm{ml}, 97.8 \mathrm{mmol}, 6\right.$ equiv) in $100 \mathrm{ml}$ acetonitrile at $0^{\circ} \mathrm{C}$, after completion of the addition, the reaction mixture was warmed to room temperature, stirred 2 $\mathrm{h}$, and then refluxed for $3 \mathrm{~h}$. Upon cooling the white solid hydochloride salt was precipitated and was removed by filtration, and the resulting solution was concentrated in vacuum to give colourless oil, that was purified using column chromatography (silica, MeOH: $\mathrm{OEt}_{2}$ 60:40).

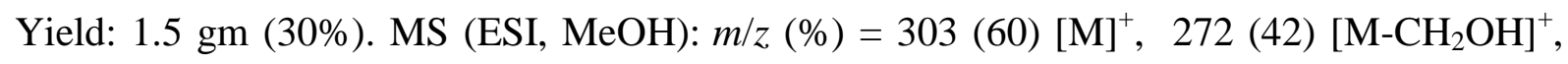
$257(100)\left[\mathrm{M}_{-} \mathrm{C}_{2} \mathrm{H}_{6} \mathrm{O}\right]^{+} .{ }^{1} \mathrm{H}$ NMR (200 MHz, DMSO): $\delta 5.1\left(\mathrm{br}, 6 \mathrm{H}, \mathrm{NH}_{2}\right), 3.66(\mathrm{~m}, 4 \mathrm{H}$, $\left.\underline{\mathrm{CH}}_{2} \underline{\mathrm{CH}}_{2}\right)$ ppm. IR (KBr): ขึ 3388 (br), 3193 (br), 2945 (w), 2129 (w), 1604 (br), 1397 (w), 1359 (w), 1231 (m), 1225 (w), 1063 (m), 994 (w), 948 (w), 864 (m), 801 (m), 730 (m), 704 (w), $614(\mathrm{w}) \mathrm{cm}^{-1}$.

2,4,6-Tris[( $N$-methyl- $N^{\prime}$-salicylaldimino)hydrazino]-1,3,5-triazine $\quad\left(\mathbf{H}_{3} \mathrm{~L}^{\mathbf{3}}\right):$ A stirred solution of 2,4,6-Tris( $N$-methylhydrazino)-1,3,5-triazine 1 (1.3 g, $6 \mathrm{mmol}, 1$ equiv) and salicylaldehyde (2.2 g, $18.0 \mathrm{mmol}, 3$ equiv) in methanol $(75 \mathrm{ml})$ was refluxed for $12 \mathrm{~h}$, during which a white precipitate formed. This was filtered off, washed with methanol, dried in air. Yield. $40 \%$. Mp > $360^{\circ} \mathrm{C}$. MS (EI): $m / z$ (\%) $525.2(60)[\mathrm{M}]^{+}$. This compound is sparingly soluble only in hot DMSO. Therefore NMR-spectroscopy is not done. 


\section{2,4,6-Tris[ $\left[N\right.$-(2-hydroxyethyl)- $N^{\prime}$-(3,5-di-tert-butylsalicylaldimino) $\}$ hydrazino]-1,3,5-}

triazine $\left(\mathbf{H}_{6} \mathbf{L}^{4}\right)$ : A stirred solution of 2,4,6-Tris $\{N$-(2-hydroxyethylhydrazino) $\}$-1,3,5-triazine 1 (1.82 g, 6 mmol, 1 equiv) and salicylaldehyde ( $2.2 \mathrm{~g}, 18.0 \mathrm{mmol}, 3$ equiv) in methanol (75 $\mathrm{ml}$ ) was refluxed for $12 \mathrm{~h}$, during which a white precipitate formed. This was filtered off, washed with methanol, and dried in air. Yield. 25\%. Mp > 300 C. MS (EI): $m / z(\%) 615$ (40) $[\mathrm{M}]^{+} .{ }^{1} \mathrm{H}$ NMR (500 MHz, DMSO- $d_{6}$ ): $\delta 12.50$ (br, 3H, Ar-OH), 8.42 (s, 3H, N=C-H), 7.32$7.28(\mathrm{~m}, 6 \mathrm{H}), 6.98-6.87(\mathrm{~m}, 6 \mathrm{H}), 4.85\left[\mathrm{br}, 3 \mathrm{H},\left(\mathrm{CH}_{2}\right)_{2} \mathrm{OH}\right], 4.43\left[\mathrm{t}, 6 \mathrm{H}, \mathrm{CH}_{2} \underline{\mathrm{CH}}_{2} \mathrm{OH}\right), 3.80$ (s, $\left.6 \mathrm{H}, \underline{\mathrm{CH}}_{2} \mathrm{CH}_{2} \mathrm{OH}\right), 1.46\left[\mathrm{~s}, 27 \mathrm{H}, \mathrm{C}\left(\mathrm{CH}_{3}\right)_{3}\right], 1.31\left[\mathrm{~s}, 27 \mathrm{H}, \mathrm{C}\left(\mathrm{CH}_{3}\right)_{3}\right] \mathrm{ppm} .{ }^{13} \mathrm{C} \mathrm{NMR}(125.75$ $\left.\mathrm{MHz}, \mathrm{DMSO}-d_{6}\right): \delta 164$ (triazine-C), $157.5(\mathrm{~N}=\mathrm{C}-\mathrm{H}), 142.7,130.6,130.4,118.9,118.8$, 116.5, 58.89, 44.3 ppm. Elemental analysis (\%) calculated for $\mathrm{C}_{30} \mathrm{H}_{33} \mathrm{~N}_{9} \mathrm{O}_{6}$ (615.6): C-58.53, H-5.40, N-20.48; found: C-57.43, H-4.67, N-19.14.

\section{2,4,6-Tris $\left[\left\{N\right.\right.$-methyl- $N^{\prime}$-(3,5-di-tert-butylsalicylaldimino) $\}$ hydrazino]-1,3,5-triazine}

$\left(\mathbf{H}_{3} \mathbf{L}^{\mathbf{5}}\right)$ : A stirred solution of 2,4,6-Tris( $N$-methylhydrazino)-1,3,5-triazine $\mathbf{1}$ (1.3 g, $6 \mathrm{mmol}, 1$ equiv) and 3,5-di-tert-butylsalicylaldehyde (4.22 g,18.0 mmol, 3 equiv) in methanol $(75 \mathrm{ml})$ was refluxed for $12 \mathrm{~h}$, during which a white precipitate formed. This was filtered off, washed with methanol, and dried in air. Yield: $1.6 \mathrm{~g}$ (28\%). Mp $360^{\circ} \mathrm{C}$. MS (EI): $\mathrm{m} / z$ (\%) 861.6 (100) $\left[{\left.\mathrm{M}-\mathrm{H}^{+}\right] .}^{1} \mathrm{H}\right.$ NMR (200 MHz, $\left.\mathrm{CDCl}_{3}\right): \delta 13.2$ (br, 3H,OH), 7.90 (s, 3H, N=C-H), 7.38 (d, 2H, C-6), 7.07 (d, 3H, C-4), 3.68 (s, 9H, Me), 1.51 [s, 27H, C( $\left.\left(\mathrm{CH}_{3}\right)_{3}\right], 1.35$ [s, 27H, C(CH $\left.\mathrm{CH}_{3}\right]$ ppm. ${ }^{13} \mathrm{C}\left\{{ }^{1} \mathrm{H}\right\}$ NMR (75.47 MHz, $\left.\mathrm{CDCl}_{3}\right): \delta 165$ (triazine-C), $155.6(\mathrm{~N}=\mathrm{C}-\mathrm{H}), 141.6(\mathrm{C} 2)$, 140.6 (C5), 136.9 (C6), $125.7(\mathrm{C} 4), 125.2$ (C3), $118.3(\mathrm{C} 1), 35.5\left[\mathrm{C}\left(\mathrm{CH}_{3}\right)_{3}\right], 34.4\left[\mathrm{C}\left(\mathrm{CH}_{3}\right)_{3}\right]$, $32\left(\mathrm{CH}_{3}\right), 29\left[\mathrm{C}\left(\mathrm{CH}_{3}\right)_{3}\right], 28.8\left[\mathrm{C}\left(\mathrm{CH}_{3}\right)_{3}\right]$ ppm. IR (KBr): ṽ $3428(\mathrm{br}), 2956(\mathrm{~m}), 2907(\mathrm{w})$, 2909 (w), 1555 (s), 1484 (s), 1426 (s), 1396 (s), 1347 (s), 1279 (m), 1232 (w), 1189 (w), 1155

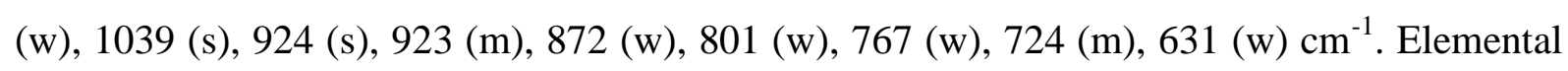
analysis (\%) calculated for $\mathrm{C}_{51} \mathrm{H}_{75} \mathrm{~N}_{9} \mathrm{O}_{3}$ (862.2): C-71.04, H-8.77, N-14.62; found: C-70.89, H-8.41, N-14.51. Single crystals were obtained on slow cooling of chlorobenzene solution. The solid state structure of the ligand $\mathbf{H}_{\mathbf{3}} \mathbf{L}^{\mathbf{5}}$ (Chapter 3.1) was determined by X-ray diffraction.

\section{2,4,6-Tris[ $\left\{N\right.$-(2-hydroxyethyl)- $N^{\prime}$-(3,5-di-tert-butylsalicylaldimino) $\}$ hydrazino]-1,3,5-}

triazine $\left(\mathbf{H}_{\mathbf{6}} \mathbf{L}^{\mathbf{6}}\right)$ : A stirred solution of 2,4,6-Tris $\{N$-(2-hydroxyethylhydrazino) $\}$-1,3,5-triazine 1 (1.82 g, 6 mmol, 1 equiv) and 3,5-di-tert-butylsalicylaldehyde (4.22 g,18.0 mmol, 3 equiv) in methanol $(75 \mathrm{ml})$ was refluxed for $12 \mathrm{~h}$, during which a white precipitate formed. This was filtered off, washed with methanol, dried in air. Yield: $1.45 \mathrm{~g}(25 \%)$. Mp $321^{\circ} \mathrm{C}$. MS (EI): $\mathrm{m} / \mathrm{z}$ (\%) $951.6(100)[\mathrm{M}]^{+} .{ }^{1} \mathrm{H}$ NMR (200 MHz, DMSO-d $)$ : $\delta 13.19$ (br, 3H, Ar-OH), 8.58 (s, 3H, 
$\mathrm{N}=\mathrm{C}-\mathrm{H}), 7.28(\mathrm{~m}, 6 \mathrm{H}, \mathrm{C} 2 \& \mathrm{C} 4), 4.89\left[\mathrm{t}, 3 \mathrm{H},\left(\mathrm{CH}_{2}\right)_{2} \underline{\mathrm{OH}}\right], 4.54\left[\mathrm{t}, 6 \mathrm{H}, \mathrm{CH}_{2} \underline{\mathrm{CH}}_{2} \mathrm{OH}\right), 3.90$ (t, $\left.6 \mathrm{H}, \underline{\mathrm{CH}_{2}} \mathrm{CH}_{2} \mathrm{OH}\right), 1.46\left[\mathrm{~s}, 27 \mathrm{H}, \mathrm{C}\left(\mathrm{CH}_{3}\right)_{3}\right], 1.31\left[\mathrm{~s}, 27 \mathrm{H}, \mathrm{C}\left(\mathrm{CH}_{3}\right)_{3}\right] \mathrm{ppm}$. This compound is sparingly soluble only in hot DMSO. Due to the solubility problem, ${ }^{13} \mathrm{C} \mathrm{NMR}$ did not measure for this compound, however upon cooling the hot DMSO solution, instantly white block shaped nice crystal was isolated in the NMR tube. IR (KBr): v 3583 (w), 3457 (br), 2952 (s), 2905 (w), 2909 (w), 1553 (s), 1448 (s), 1429 (s), 1386 (m), 1342 (s), 1267 (m), 1249 (w), 1231 (w), 1203 (m), 1172 (m), 1132 (w), 1042 (br), 986 (w), 923 (w), 867 (w), 819 (m), $802(\mathrm{w}), 768(\mathrm{w}), 722(\mathrm{w}), 640(\mathrm{w}) \mathrm{cm}^{-1}$. Elemental analysis (\%) calculated for $\mathrm{C}_{54} \mathrm{H}_{81} \mathrm{~N}_{9} \mathrm{O}_{6}$ (952.3): C-68.11, H-8.57, N-13.24; found: C-67.43, H-8.67, N-13.14.

\section{2,4,6-Tris $\left[\left\{N\right.\right.$-methyl- $N^{\prime}$-(pyridine-2-yl)methylidene $\}$ hydrazino $]-1,3,5$-triazine $\quad\left(\mathrm{L}^{7}\right)$ : $\mathrm{A}$} stirred solution of 2,4,6-Tris( $N$-methylhydrazino)-1,3,5-triazine 1 (1.3 gm, $6 \mathrm{mmol}, 1$ equiv) and pyridine- 2 carboxaldehyde $(1.73 \mathrm{ml}, 18.0 \mathrm{mmol}, 3$ equiv) in methanol was refluxed for 12 $\mathrm{h}$, during which a white precipitate formed. This was filtered off, washed with methanol, dried in air. Yield: $2.70 \mathrm{gm}(94 \%)$. MS (EI): $m / z(\%)=481(40)[\mathrm{M}+\mathrm{H}]^{+}, 480(100)[\mathrm{M}]^{+} .{ }^{1} \mathrm{H}$ NMR (500 MHz, DMSO- $\left.d_{6}\right): \delta 8.62(\mathrm{~s}, 3 \mathrm{H}), 8.13(\mathrm{~d}, 3 \mathrm{H}), 8.01(\mathrm{~d}, 3 \mathrm{H}), 7.93(\mathrm{~m}, 3 \mathrm{H}), 7.4(\mathrm{~m}, 3 \mathrm{H})$, $3.78(\mathrm{~s}, 3 \mathrm{H}) \mathrm{ppm} .{ }^{13} \mathrm{C}\left\{{ }^{1} \mathrm{H}\right\} \mathrm{NMR}\left(125.75 \mathrm{MHz}\right.$, DMSO-d $\left.d_{6}\right): \delta \quad 165.2,154.4,149.3,139.7$, 136.7, 123.6, 119.2, 30.9 ppm. IR (KBr): ṽ 1586 (w), 1545 (m), 1517(w), $1483(\mathrm{w}), 1462$ (m), 1417(m), 1389 (m), 1333 (m), 1289 (w), 1213 (w), 1167 (m), 1033 (s), 987 (w), 912 (w), 774 (w), $741(\mathrm{w}), 625(\mathrm{w}) \mathrm{cm}^{-1}$. Elemental analysis (\%) calculated for $\mathrm{C}_{24} \mathrm{H}_{24} \mathrm{~N}_{12}$ (480.2): C-59.99, H-5.03, N-34.98; found: C-58.92, H-5.44, N-35.05.

2-[3-(2-Hydroxyphenyl)-1H-pyrazol-1-yl]ethanol $\quad\left(\mathbf{H}_{2} \mathbf{L}^{9}\right):$ A mixture of 1-(2hydroxyphenyl)-3-dimethylaminoprop-2-enone (20 g, $0.104 \mathrm{~mol})$, and 2-hydroxyethyl hydrazine $(29.3 \mathrm{~g}, 0.384 \mathrm{~mol})$ in $100 \mathrm{ml}$ methanol was heated to reflux with stirring for $3 \mathrm{~h}$. Then the methanolic solution was poured on crushed ice, and the resulting brown precipitate was isolated by filtration and dried in air. The crude product was purified by soxhlet apparatus with petroleum ether over several days to give $\mathbf{H}_{2} \mathbf{L}^{\mathbf{9}}$ as needle-shaped white crystals. Yield: $9.3 \mathrm{~g}(45 \%) . \mathrm{Mp} 91^{\circ} \mathrm{C}$. MS (EI): $m / z(\%)=204(100)[\mathrm{M}]^{+}, 173(80)\left[\mathrm{M}-\mathrm{CH}_{2} \mathrm{OH}\right]^{+}, 160(40)$ $\left[\mathrm{M}-\mathrm{CH}_{2} \mathrm{CH}_{2} \mathrm{O}\right]^{+} .{ }^{1} \mathrm{H}$ NMR (500 MHz, DMSO-d $\left.)_{6}\right): \delta 10.68(\mathrm{~s}, \mathrm{Ar}-\mathrm{OH}), 7.84(\mathrm{~d}, 1 \mathrm{H}, J=2.5$ $\left.\mathrm{Hz}, 5-\mathrm{H}_{\mathrm{pz}}\right), 7.70(\mathrm{dd}, 1 \mathrm{H}, J=7.5,1.5 \mathrm{~Hz}, \mathrm{ArH}), 7.15$ (ddd, $1 \mathrm{H}, J=1.5,6.75$, and $8.5 \mathrm{~Hz}$, ArH), $6.89(\mathrm{dd}, 1 \mathrm{H}, J=1 \mathrm{~Hz}, 8 \mathrm{~Hz}, \mathrm{ArH}), 6.87$ (dd, 1H, J=1 Hz, $7.5 \mathrm{~Hz}, \mathrm{ArH}), 6.85$ (d, 1H, $\left.J=1 \mathrm{~Hz}, 4-\mathrm{H}_{\mathrm{pz}}\right), 4.93\left(\mathrm{t}, 1 \mathrm{H}, J=5 \mathrm{~Hz}, \mathrm{CH}_{2} \mathrm{CH}_{2} \underline{\mathrm{OH}}\right.$ ), 4.22 (t, $\left.2 \mathrm{H}, J=5.5 \mathrm{~Hz}, \mathrm{CH}_{2} \underline{\mathrm{CH}}_{2} \mathrm{OH}\right)$, 3.77 (q, 2H, $\left.J=5.5 \mathrm{~Hz}, \underline{\mathrm{CH}}_{2} \mathrm{CH}_{2} \mathrm{OH}\right) \mathrm{ppm} .{ }^{13} \mathrm{C}\left\{{ }^{1} \mathrm{H}\right\}$ NMR (125.75 MHz, DMSO- $\left.d_{6}\right): \delta 154.9$, 
149.7, 132.1, 128.6, 126.6, 119.2, 117.2, 116.4, 102.5, 59.8, 54.1 ppm. IR (KBr): ṽ 33478 (w), 3325 (br), 3015 (w), 2958 (w), 2926 (w), 2871 (w), 1620 (m), 1584 (m), 1508 (m), 1487 (m), 1466 (m), 1430 (w), 1415 (m), 1360 (w), 1350 (w), 1317 (w), 1291 (m), 1248 (s), 1211 (w), 1152 (w), 1080 (m), 1059 (s), 1045 (w), 935 (m), 861 (m), 825 (m), 782 (m), 758 (m), $702(\mathrm{~m}), 702(\mathrm{~m}), 675(\mathrm{~m}) \mathrm{cm}^{-1}$. Elemental analysis (\%) calculated for $\mathrm{C}_{11} \mathrm{H}_{12} \mathrm{~N}_{2} \mathrm{O}_{2}$ (204.2): C64.69, H-5.92, N-13.72; found: C-64.71, H-6.05, N-13.73.

2-[\{3-(2-Hydroxyphenyl)-5-phenyl\}-1H-pyrazol-1-yl]ethanol $\left(\mathbf{H}_{2} \mathbf{L}^{10}\right):$ It was obtained in similar manner as described for $\mathbf{H}_{2} \mathbf{L}^{9}$, but using starting precosour used 1-(2-hydroxy phenyl)-3-phenylpropane-1,3-dione instead of 1-(2-hydroxyphenyl)-3-dimethylaminoprop-2enone. Yield $\sim 5 \%$. MS (EI): $m / z(\%)=279.1(100)\left[\mathrm{M}-\mathrm{H}^{+}\right] .{ }^{1} \mathrm{H}$ NMR $\left(200 \mathrm{MHz}, \mathrm{CDCl}_{3}\right): \delta$ 10.70 (s, Ar-OH), 7.57 (dd, 1H, ArH), 7.52-7.47 (m, 5H, Ph), 7.20 (dd, 1H, ArH), 7.08 (dd, $1 \mathrm{H}, \mathrm{ArH}), 6.96(\mathrm{dt}, 1 \mathrm{H}, \mathrm{ArH}), 6.69\left(\mathrm{~s}, 1 \mathrm{H}, 4-\mathrm{H}_{\mathrm{pz}}\right), 4.30\left(\mathrm{t}, 2 \mathrm{H}, \mathrm{CH}_{2} \underline{\mathrm{CH}_{2}} \underline{\mathrm{OH}}\right), 4.10(\mathrm{t}, 2 \mathrm{H}, J=$ $\left.5.5 \mathrm{~Hz}, \underline{\mathrm{CH}}_{2} \mathrm{CH}_{2} \mathrm{OH}\right)$.

R-2-[1-(2-Hydroxy-2-phenylethyl)-1H-pyrazol-3-yl]-phenol ( $\left.R-\mathbf{H}_{2} \mathbf{L}^{11}\right)$ : It was synthesized by modifications of previously reported procedure. A solution of 3-(2methoxyphenyl)pyrazole $(1.0 \mathrm{mmol})$ in the mixture of toluene-DMF $(2: 0.5 \mathrm{ml} / \mathrm{ml})$ was added to the stirred suspension of sodium hydride $(1.1 \mathrm{mmol})$ in DMF $(0.5 \mathrm{ml})$ under $\mathrm{N}_{2}$ atmosphere at room temperature. The mixture was heated to $60^{\circ} \mathrm{C}$, stirred for the next $1.5 \mathrm{~h}$ and cooled to $25^{\circ} \mathrm{C}$. Reaction flask was placed in a water bath and the solution of $R-(+)$-styrene oxide (120 $\mathrm{mg}, 1.0 \mathrm{mmol}$ ) in DMF (2 ml) was added via syringe for $25 \mathrm{~min}$. After $1 \mathrm{~h}$ temperature was raised to $60^{\circ} \mathrm{C}$ and the reaction mixture was stirred under inert atmosphere for $24 \mathrm{hrs}$. The reaction was con trolled by TLC (silica gel, $\mathrm{CHCl}_{3} /$ hexane, 6:4, v/v). Then, solvents were evaporated in vacuo and the residue was diluted with water and $\mathrm{CH}_{2} \mathrm{Cl}_{2}$, the resulted phases were separated and water layer was washed with dichloromethane. Combined organic fractions were dried over $\mathrm{MgSO}_{4}$; solvent was removed in vacuo giving red oil.

To a solution of $\mathbf{M e}_{2} \mathbf{L}^{\mathbf{1 1}}$ (4.2 g, 1 equiv.) in dry dicholormethane $(200 \mathrm{ml})$ under $\mathrm{N}_{2}$ at $-80^{\circ} \mathrm{C}$ (dry ice/ethanol) was added $\mathrm{BBr}_{3}$ as $1 \mathrm{M}$ solution in dicholormethane (5 equiv.). The mixture was warmed to room temperature and stirred overnight. After this period aqueous $\mathrm{NaOH}(\mathrm{pH}$ 9) was carefully added until the evaluation of gas ceased. The mixture was extracted with dichloromethane, and the combined organic extracts were dried $\left(\mathrm{MgSO}_{4}\right)$. Removal of the solvent afforded a reddish yellow oily product formed, which was purified by column chromatography (silica, $\mathrm{CHCl}_{3} /$ hexane $6: 4$ ) to yield $\mathrm{R}-\mathrm{H}_{2} \mathrm{~L}$ as a white solid (70\%). MS (EI): 
$m / z(\%)=263.1(80),[\mathrm{M}-\mathrm{OH}]^{+} .{ }^{1} \mathrm{H} \mathrm{NMR}\left(500 \mathrm{MHz}, \mathrm{CDCl}_{3}\right): \delta 10.53(\mathrm{~s}, \mathrm{Ar}-\mathrm{OH}), 7.50(\mathrm{dd}$, 1H, 7.6 Hz, 1.7 Hz, ArH), 7.40-7.28 (m, 5H, Ph), 7.22 (d, 1H, 1.9 Hz, 5- $\left.\mathrm{H}_{\mathrm{pz}}\right), 7.18$ (d, 1H, $1.25 \mathrm{~Hz}, \operatorname{ArH}), 7.00$ (dd, 1H, 8.4 Hz, 1.2 Hz, ArH), 6.85 (dt, 1H, 8.1 Hz, 1.6 Hz, ArH), 6.48 (d, 1H, $\left.2.52 \mathrm{~Hz}, 4-\mathrm{H}_{\mathrm{pz}}\right), 5.31$ (t, 2H, $\left.7.56 \mathrm{~Hz}, \mathrm{CH}_{2} \underline{\mathrm{CH}}(\mathrm{Ph}) \mathrm{OH}\right), 4.7$ (m, 2H, $\left.\underline{\mathrm{CH}}_{2} \mathrm{CH}(\mathrm{Ph}) \mathrm{OH}\right)$, $1.40\left(\mathrm{~s}, 1 \mathrm{H}, \mathrm{CH}_{2} \mathrm{CH}(\mathrm{Ph}) \underline{\mathrm{OH}}\right) .{ }^{13} \mathrm{C}\left\{{ }^{1} \mathrm{H}\right\} \mathrm{NMR}\left(125.75 \mathrm{MHz}, \mathrm{CDCl}_{3}\right): \delta 155.7(\mathrm{C}-\mathrm{OH}), 152.3$ $\left(\mathrm{C}-3_{\mathrm{pz}}\right), 138.1$ (C-Ar), $131.4\left(\mathrm{C}-4_{\mathrm{Ar}}\right), 129.3\left(\mathrm{C}-5_{\mathrm{pz}}\right), 129.2\left(\mathrm{C}-5_{\mathrm{Ar}}\right), 129.0(\mathrm{C}-\mathrm{Ar}), 127.6(\mathrm{C}-$ $\left.3_{\mathrm{Ar}}\right), 126.4(\mathrm{C}-\mathrm{Ar}), 119.3\left(\mathrm{C}-4_{\mathrm{Ar}}\right), 117.0\left(\mathrm{C}-2_{\mathrm{Ar}}\right), 116.4\left(\mathrm{C}-6_{\mathrm{Ar}}\right), 102.2\left(\mathrm{C}-4_{\mathrm{pz}}\right), 59.3(\mathrm{C}-\mathrm{OH})$, $51.0\left(\mathrm{CH}_{2}\right)$.

3,5-Bis[(3,5-di-tert-butylsalicylaldimino)methyl]pyrazole $\quad\left(\mathbf{H}_{\mathbf{3}} \mathbf{L}^{\mathbf{1 2}}\right): \quad 3,5$-Bis(aminomethyl) pyrazole dihydrochloride $(1 \mathrm{gm}, 10 \mathrm{mmol})$ was added in methanol $(50 \mathrm{~mL})$ in $250 \mathrm{~mL}$ flask, and the suspension solution was neutralised by solid $\mathrm{NaOH}$ (881 mg, $22 \mathrm{mmol}$ ), stirred 10-15 mints at room temperature, and then filtered to remove some undesired inorganic salt. To this clear colourless solution was added drop wise 3,5-di-tert-butyl-2-hydroxy benzaldehyde (4.68 $\mathrm{gm}, 20 \mathrm{mmol}$ ) in $25 \mathrm{~mL}$ methanol at room temperature, and then refluxed $3 \mathrm{hrs}$. After completion of the reaction, removal of the solvent through the vacuo, yielded $\mathbf{H}_{\mathbf{3}} \mathbf{L}^{\mathbf{1 2}}$ as a yellow solid (5.50 gm, $9.85 \mathrm{mmol}, 98 \%)$. MS (EI): $m / z(\%)=559.3(42)[\mathrm{M}+\mathrm{H}]^{+}, 558.3(100)$ $[\mathrm{M}]^{+} .{ }^{1} \mathrm{H}$ NMR $\left(200 \mathrm{MHz}, \mathrm{CDCl}_{3}\right): \delta(\mathrm{ppm})=1.32\left[\mathrm{~s}, 18 \mathrm{H}, \mathrm{C}\left(\mathrm{CH}_{3}\right)_{3}\right], 1.47\left[\mathrm{~s}, 18 \mathrm{H}, \mathrm{C}\left(\mathrm{CH}_{3}\right)_{3}\right]$, $4.78\left(\mathrm{~s}, 4 \mathrm{H}, 2 \times \mathrm{CH}_{2}\right), 6.20\left(\mathrm{~s}, 1 \mathrm{H}, \mathrm{CH}^{\mathrm{pz}}\right), 7.10(\mathrm{~d}, 2 \mathrm{H}, \mathrm{C}-4), 7.45$ (d, 2H, C-6), $8.40(\mathrm{~s}, 2 \mathrm{H}$, $\mathrm{N}=\mathrm{C}-\mathrm{H}), 9.9$ (br, 1H, NH), 13.65 (br, 2H, OH). IR (KBr): ṽ 3412 (br), 2958 (s), 2907 (w), 2869 (w), 1629 (s), 1585 (m), 1468 (m), 1440 (m), 1361 (m), 1273 (m), 1251 (m), 1202 (w), 1173 (m),1045 (w), 927 (w), 875 (w), 804 (w), 771 (w), 725 (w), 648 (w) cm $\mathrm{cm}^{-1}$. Elemental analysis (\%) calculated for $\mathrm{C}_{35} \mathrm{H}_{50} \mathrm{~N}_{4} \mathrm{O}_{2}$ (558.8): C-75.23, H-9.02, N-10.03; found: C-74.89, H-8.91, N-9.04.

\subsection{Synthesis of metal complexes and cluster compounds:}

Synthesis of $\left[\left\{\mathrm{L}^{5 \mathrm{a}} \mathrm{Cu}^{\mathrm{II}}{ }_{2}\left(\mathrm{H}_{2} \mathrm{O}\right)_{2}(\mathrm{MeOH})_{2}\right\}\left\{\mathrm{L}^{5 \mathrm{a}} \mathrm{Cu}_{2}(\mathrm{MeOH})_{4}\right\}\right]\left(\mathrm{NO}_{3}\right)_{2} \cdot \mathrm{xCH}_{3} \mathrm{OH} \cdot \mathrm{yH}_{2} \mathrm{O}(3)$ : A solution of $\mathrm{Cu}\left(\mathrm{NO}_{3}\right)_{2} \cdot \mathrm{xH}_{2} \mathrm{O}(76 \mathrm{mg}, 0.315 \mathrm{mmol})$ in methanol $(15 \mathrm{ml})$ was added to a stirred solution of $\mathbf{H}_{\mathbf{3}} \mathbf{L}^{\mathbf{5}}$ (100 $\left.\mathrm{mg}, 0.105 \mathrm{mmol}\right)$ in chloroform $(50 \mathrm{ml})$ resulting in a green solution. A solution of $\mathrm{NEt}_{3}(64 \mathrm{mg}, 0.63 \mathrm{mmol})$ in methanol $(5 \mathrm{ml})$ was added drop wise at room temperature, stirred overnight, the resulting dark green solution evaporated to dryness under reduced pressure to give a green crystalline material, washed with acetonitrile $(10 \mathrm{ml})$ to remove the undesired product, and then dissolved in methanol $(15 \mathrm{ml})$, dark green crystals of 3 were obtained using slow evaporation of the resulting solution. Yield: $30 \mathrm{mg}$ (34\%). MS 


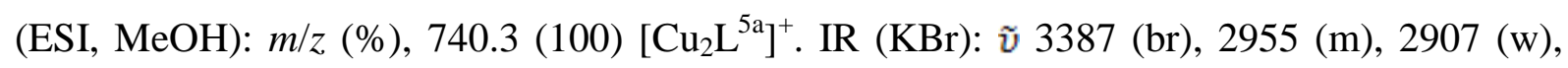
2869 (w), 1606 (w), 1545 (s), 1527 (m), 1437 (w), 1384 (s), 1255 (w), 1230 (w), 1200 (s), 1057 (s), 932 (w), 837 (w), $784(w), 746$ (w), 701 (w), 614 (w) $\mathrm{cm}^{-1}$. Elemental analysis (\%) calculated for $\mathrm{C}_{35} \mathrm{H}_{48} \mathrm{Cu}_{2} \mathrm{~N}_{8} \mathrm{O}_{6}$ (803.9): C-52.29, H-6.02, N-13.94; found: C-51.99, H-6.20, N13.54 .

Synthesis of $\left[\mathrm{Mn}^{\mathrm{III}}{ }_{4}\left(\mathrm{H}_{2} \mathrm{~L}^{6 \mathrm{a}}\right)_{4}\right] \cdot \mathbf{x C H}_{3} \mathbf{C N} \cdot \mathbf{y C H} \mathbf{C H}_{3} \mathrm{OH}$ (4): A solution of $\mathrm{Mn}\left(\mathrm{NO}_{3}\right)_{2} \cdot \mathrm{xH}_{2} \mathrm{O}(56$ $\mathrm{mg}, 0.315 \mathrm{mmol})$ in methanol $(20 \mathrm{ml})$ was added to a stirred solution of $\mathbf{H}_{\mathbf{6}} \mathbf{L}^{\mathbf{6}}(100 \mathrm{mg}, 0.105$ $\mathrm{mmol})$ in chloroform $(50 \mathrm{ml})$ resulting in an yellow solution. A solution of $\mathrm{NEt}_{3}(64 \mathrm{mg}, 0.63$ mmol) in methanol $(5 \mathrm{ml})$ was added drop wise at room temperature, refluxed overnight, the resulting solution was evaporated to dryness under reduced pressure to give a red solid, then dissolved in (2:1) acetonitrile-methanol $(20 \mathrm{ml})$, red crystals of 4 were obtained by slow evaporation of the resulting solution. Yield: $6 \mathrm{mg}(8 \%)$. Mp $240^{\circ} \mathrm{C}$. HRESI-MS $(\mathrm{MeOH}): \mathrm{m} / \mathrm{z}$ (\%) 2918.4 (34) $\left[\mathrm{Mn}_{4}\left(\mathrm{H}_{2} \mathrm{~L}^{6 \mathrm{a}}\right)_{4}-\mathrm{H}\right]^{+}, 1459.7$ (64) $\left[\mathrm{Mn}_{4}\left(\mathrm{H}_{2} \mathrm{~L}^{6 \mathrm{a}}\right)_{4}\right]^{2+}$. IR (KBr): vै 3430 (br), 2980 (w), 2842 (w), 1655 (w), 1610 (w), 1523 (m), 1503 (w), 1462 (w), 1383 (s), $1250(\mathrm{w}), 1171$ (w), $1052(\mathrm{~m}), 835(\mathrm{w}) \mathrm{cm}^{-1}$. Elemental analysis (\%) calculated for $\mathrm{C}_{148} \mathrm{H}_{208} \mathrm{Mn}_{4} \mathrm{~N}_{28} \mathrm{O}_{20}$ (2919.2): C-60.89, H-7.18, N-13.43; found: C-60.09, H-7.00, N-13.03.

Synthesis of $\left[\mathrm{Fe}^{\mathrm{III}}{ }_{4}\left(\mu_{2}-\mathrm{O}\right)\left(\mathrm{H}_{2} \mathrm{O}\right)_{4}\left(\mathrm{H}_{2} \mathrm{~L}^{6 \mathrm{a}}\right)_{2}\left(\mathrm{~L}^{\mathrm{t}-\mathrm{Bu}}{ }_{2}\right)_{2}\right]\left(\mathrm{NO}_{3}\right)_{2} \cdot 3 \mathrm{CH}_{3} \mathrm{OH} \cdot 6 \mathrm{CH}_{3} \mathrm{CN}$ (5): A solution of $\mathrm{Fe}\left(\mathrm{NO}_{3}\right)_{3} \cdot 9 \mathrm{H}_{2} \mathrm{O}(127 \mathrm{mg}, 0.315 \mathrm{mmol})$ in methanol $(20 \mathrm{ml})$ was added to a stirred solution of $\mathbf{H}_{\mathbf{6}} \mathbf{L}^{\mathbf{6}}$ (100 mg, $\left.0.105 \mathrm{mmol}\right)$ in chloroform $(50 \mathrm{ml})$ resulting in a black solution. A solution of $\mathrm{NEt}_{3}(64 \mathrm{mg}, 0.63 \mathrm{mmol})$ in methanol $(5 \mathrm{ml})$ was added dropwise at room temperature, refluxed overnight, the resulting solution was evaporated to dryness under reduced pressure to give a black crystalline material, dissolved in acetonitrile $(20 \mathrm{ml})$, filtered, black crystals of $\mathbf{5}$ were separated by slow evaporation of the filtrate. Yield: $5 \mathrm{mg}(7 \%)$. Mp $>300^{\circ} \mathrm{C}$. HRESI-MS

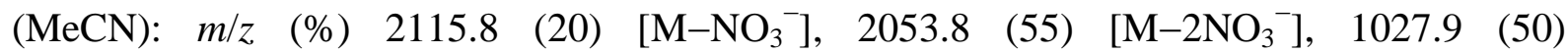
$\left[\mathrm{M}-2 \mathrm{NO}_{3}+\mathrm{H}\right]^{2+}$. IR (KBr): ขึ 3430 (br), 2957 (w), 2922 (w), $1620(\mathrm{w}), 1595$ (w), 1532 (m), 1491 (w), 1477 (w), 1383 (s), 1361 (w), 1273 (w), 1255 (w), 1171 (w), 1052 (m), 843 (w), $799(\mathrm{w}), 778(\mathrm{w}) \mathrm{cm}^{-1}$. Elemental analysis (\%) calculated for $\mathrm{C}_{121} \mathrm{H}_{186} \mathrm{Fe}_{4} \mathrm{~N}_{20} \mathrm{O}_{28}(\mathbf{5} ; 2592.3)$ : C-56.06, H-7.23, N-10.81; found: C-55.09, H-7.00, N-9.93.

Preparation of $\left[\mathrm{Mn}^{\mathrm{II}}\left(\mathrm{L}^{7}\right)\left(\mathrm{NO}_{3}\right)\left(\mathrm{H}_{2} \mathrm{O}\right)\right]\left(\mathrm{NO}_{3}\right) \cdot \mathbf{x C H}_{3} \mathrm{OH}(6)$ : A solution of ligand $\mathbf{L}^{7}$ (100 mg, $0.208 \mathrm{mmol})$ and $\mathrm{Mn}\left(\mathrm{NO}_{3}\right)_{2} \cdot 4 \mathrm{H}_{2} \mathrm{O}(52 \mathrm{mg}, 0.208 \mathrm{mmol})$ in methanol $(20 \mathrm{ml})$ were stirred for $1 \mathrm{~h}$ at room temperature. Light yellow crystals were obtained by slow evaporation of the 
resulting solution. Yield: $87 \mathrm{mg}$ (62\%). IR (KBr): ขึ 3420 (br), 1577 (w), 1564 (w), 1529 (w), 1490 (m), 1466 (m), 1421 (m), 1384 (s), [ข̃ $\left(\mathrm{NO}_{3}{ }^{-}\right)$], 1356 (w), 1278 (w), 1170 (w), 1033 (m), $905(\mathrm{w}), 801(\mathrm{w}), 778(\mathrm{w}) \mathrm{cm}^{-1}$. MS (ESI, MeOH): $m / z(\%)=597.1(36)\left[\mathrm{L}^{7} \mathrm{Mn}\left(\mathrm{NO}_{3}\right)\right]^{+}$. Elemental analysis (\%) calculated for $\mathrm{C}_{24} \mathrm{H}_{26} \mathrm{MnN}_{14} \mathrm{O}_{7}$ (677.5): C-42.55, H-3.87, N-28.94; found: C-42.13, H-4.16, N-28.51.

Preparation of $\left[\mathrm{Mn}^{\mathrm{II}}{ }_{2}\left(\mathrm{~L}^{7}\right)_{2}\left(\mathbf{N}_{3}\right)\left(\mathrm{NO}_{3}\right)\left(\mathrm{H}_{2} \mathrm{O}\right)\right]\left(\mathrm{NO}_{3}\right)_{2} \cdot \mathbf{x H}_{2} \mathrm{O}$ (7): 2.3 equivalent of solid $\mathrm{Mn}\left(\mathrm{NO}_{3}\right)_{2} \cdot 4 \mathrm{H}_{2} \mathrm{O}(60 \mathrm{mg}, 0.260 \mathrm{mmol})$ was added to 1 equivalent of ligand $\mathbf{L}^{7}$ (50 mg, .104 $\mathrm{mmol})$ in methanol $(50 \mathrm{ml})$.The reaction mixture was stirred for $1 \mathrm{~h}$ at room temperature, and then 1 equivalent of solid sodium azide $(7 \mathrm{mg}, 0.104 \mathrm{mmol})$ was added to the reaction mixture, the light yellow solution becomes deep yellow within 5 mints, stirred for further $2 \mathrm{~h}$ at room temperature. Plate shaped yellow crystal of 7 were obtained by slow evaporation of

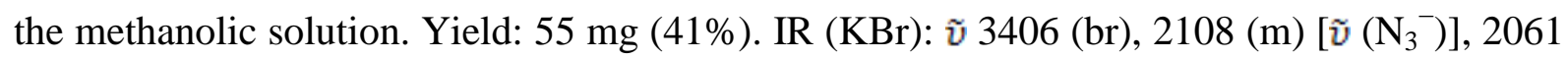

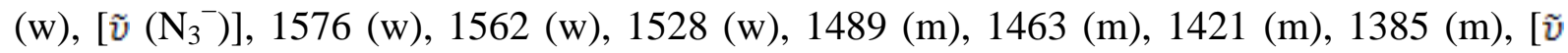
$\left(\mathrm{NO}_{3}{ }^{-}\right)$], $1355(\mathrm{~m}), 1277(\mathrm{w}), 1172(\mathrm{w}), 1034(\mathrm{~m}), 905(\mathrm{w}), 800(\mathrm{w}), 778(\mathrm{w}) \mathrm{cm}^{-1}$. Elemental analysis (\%) calculated for $\mathrm{C}_{48} \mathrm{H}_{48} \mathrm{Mn}_{2} \mathrm{~N}_{30} \mathrm{O}_{10}$ (1314.9): C-43.84, H-3.68, N-31.96; found: C43.17, H-4.04, N-32.29.

Preparation of $\left[\mathbf{M n}^{\mathrm{II}}\left(\mathbf{L}^{7}\right)\left(\mathbf{N}_{\mathbf{3}}\right)_{2}\right] \cdot \mathbf{C}_{\mathbf{2}} \mathbf{H}_{\mathbf{8}} \mathbf{O}_{\mathbf{2}}(\mathbf{8})$ : 1 equivalent of solid $\mathrm{Mn}\left(\mathrm{NO}_{3}\right)_{2} \cdot 4 \mathrm{H}_{2} \mathrm{O}(26 \mathrm{mg}$, $0.104 \mathrm{mmol})$ was added to 1 equivalent of ligand $\mathbf{L}^{7}(50 \mathrm{mg}, 0.104 \mathrm{mmol})$ in methanol (50 $\mathrm{ml})$. The reaction mixture was stirred for $1 \mathrm{~h}$ at room temperature, and then 2 equivalent of solid sodium azide (14 mg, $0208 \mathrm{mmol}$ ) was added to the reaction mixture, the light solution becomes deep yellow within 5 mints, stirred for further $2 \mathrm{~h}$ at room temperature. Plate shaped yellow crystals of $\mathbf{8}$ were obtained by slow evaporation of the methanolic solution. Yield: 55

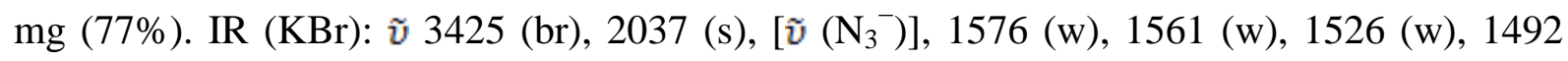
(m), 1466 (m), 1424 (m), 1398 (m), 1359 (m), 1345 (w), 1277 (w), 1168 (m), 1037 (m), 1005(w), $920(w), 906$ (w), $799(w), 782(w), 748$ (w), $694(w) \mathrm{cm}^{-1}$. Elemental analysis (\%) calculated for $\mathrm{C}_{24} \mathrm{H}_{24} \mathrm{MnN}_{18}$ (619.5): C-45.63, H-3.90, N-40.70; found: C-46.15, H-3.95, N41.14 .

Preparation of $\left[\mathbf{M n}^{\mathrm{II}}\left(\mathbf{L}^{7}\right)\left(\mathbf{N}_{3}\right)\right]_{\mathbf{n}}\left(\mathbf{N O}_{3}\right)_{\mathbf{n}} \cdot \mathbf{n C} \mathbf{C H}_{3} \mathbf{O H}(\mathbf{9})$ : 1 equivalent of solid $\mathrm{Mn}\left(\mathrm{NO}_{3}\right)_{2} \cdot 4 \mathrm{H}_{2} \mathrm{O}$ (26 mg, $0.104 \mathrm{mmol}$ ) was added to 1 equivalent of ligand $\mathbf{L}^{7}(50 \mathrm{mg}, 0.104 \mathrm{mmol})$ in methanol $(50 \mathrm{ml})$. The reaction mixture was stirred for $1 \mathrm{~h}$ at room temperature, and then 1 equivalent of solid sodium azide $(7 \mathrm{mg}, 0.107 \mathrm{mmol})$ was added to the reaction mixture, the 
light yellow solution becomes deep yellow within 5 mints, stirred for further $2 \mathrm{~h}$ at room temperature. Light yellow crystals were obtained by slow evaporation of the methanolic

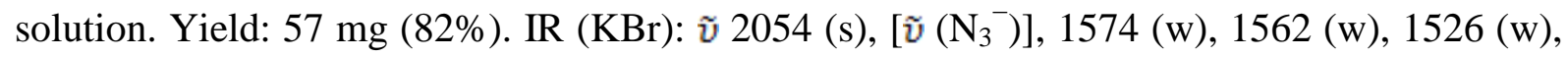
1491 (m), 1467 (m), 1422 (m), 1384 (s), [ $\widetilde{v}\left(\mathrm{NO}_{3}{ }^{-}\right)$], 1355 (m), 1342 (w), 1278 (w), $1174(\mathrm{~m})$, 1035 (m), 990 (w), 906 (w), 799 (w), 780 (w), 637 (w) $\mathrm{cm}^{-1}$. Elemental analysis (\%) calculated for $\mathrm{C}_{25} \mathrm{H}_{28} \mathrm{MnN}_{16} \mathrm{O}_{4}$ (671.6): C-44.71, H-4.20, N-33.37; found: C-43.99, H-4.12, $\mathrm{N}-34.15$.

Preparation of $\left[\mathrm{Mn}^{\mathrm{II}} \mathrm{L}^{7}(\mathbf{d c a})\left(\mathrm{H}_{2} \mathrm{O}\right)\right]\left(\mathrm{NO}_{3}\right) \cdot \mathbf{x C H}_{3} \mathrm{OH} \cdot \mathbf{x H}_{2} \mathrm{O}$ (10): 1 equivalent of solid $\mathrm{Mn}\left(\mathrm{NO}_{3}\right)_{2} \cdot 4 \mathrm{H}_{2} \mathrm{O}(26 \mathrm{mg}, 0.104 \mathrm{mmol})$ was added to 1 equivalent of ligand $\mathbf{L}^{7}$ (50 mg, 0.104 $\mathrm{mmol})$ in methanol $(50 \mathrm{ml})$. The reaction mixture was stirred for $1 \mathrm{~h}$ at room temperature, and then 1 equivalent of solid sodium dicanamide (9 $\mathrm{mg}, 0.107 \mathrm{mmol}$ ) was added to the reaction mixture, the light yellow solution becomes deep yellow within 5 mints, and then stirred for further $2 \mathrm{~h}$ at room temperature. Light yellow crystals were obtained by slow evaporation of the methanolic solution. Yield: $57 \mathrm{mg}$ (82\%). IR (KBr): ขै 3420 (br, OH), 2061 [w, v (dca)], 2215 [w, v (dca)], 2154 [(s, v (dca)], 2133 [(s, v (dca)], 1565 (m), 1563 (m), 1528

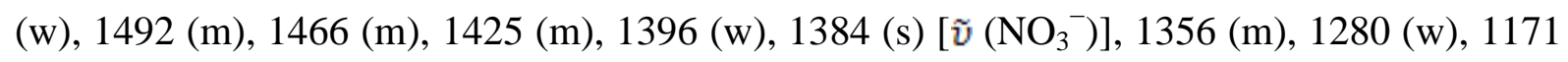
(m), $1041(\mathrm{~m}), 906(\mathrm{w}), 775(\mathrm{w}) \mathrm{cm}^{-1}$. Elemental analysis (\%) calculated for $\mathrm{C}_{26} \mathrm{H}_{26} \mathrm{MnN}_{16} \mathrm{O}_{4}$ (651.6): C-45.82, H-3.85, N-32.88; found: C-43.90, H-4.12, N-34.05.

Synthesis of $\left[\mathrm{Ni}^{\mathrm{II}}{ }_{4}(\mathbf{M e O H})_{4}\left(\mathbf{L}^{\mathbf{9}}\right)_{\mathbf{4}}\right] \cdot \mathbf{H}_{\mathbf{2}} \mathrm{O}(\mathbf{1 1}): \mathrm{Ni}(\mathrm{OAc})_{2} \cdot 4 \mathrm{H}_{2} \mathrm{O}(305 \mathrm{mg}, 1.22 \mathrm{mmol})$ was added to a solution of $\mathbf{H}_{2} \mathbf{L}^{9}$ (250 mg, $1.22 \mathrm{mmol}$ ) in $50 \mathrm{ml}$ methanol and then $\mathrm{NaOH}(98 \mathrm{mg}$, $2.44 \mathrm{mmol}$ ) in $10 \mathrm{ml}$ methanol was added drop wise to the reaction mixture, affording a green precipitate almost immediately. The green precipitate was collected by filtration, washed with methanol, and dried in air. Then resulting green powder was dissolved in $\mathrm{CH}_{2} \mathrm{Cl}_{2}(50 \mathrm{ml})$, and layered with $n$-hexane, green crystals of 11 were separated after several days.Yield: $80 \mathrm{mg}$ (22\%). MS (ESI, $\left.\mathrm{CH}_{2} \mathrm{Cl}_{2}\right): m / z(\%) 1042.9(30)\left[\left(\mathrm{L}^{9}\right)_{4} \mathrm{Ni}_{4}+\mathrm{H}\right]^{+}, 1065.9(20)\left[\left(\mathrm{L}^{9}\right)_{4} \mathrm{Ni}_{4}+\mathrm{Na}\right]^{+}$. IR (KBr): ṽ 3340 (br), 3045 (w), 2926 (w), 2866 (w), 2766 (w), 1597 (s), 1556 (s), 1518 (m), 1498 (m), 1466 (s), 1441 (m), 1415 (w), 1371 (w), 1317 (s), 1352 (m), 1211 (w), 1191 (w), 1130 (s), 1110 (w), 1070 (s), 1035 (w), 955 (w), 886 (m), 847 (m), 770 (w), 747 (s), 690 (w), 611 (w) $\mathrm{cm}^{-1}$. Elemental analysis (\%) calculated for $\mathrm{C}_{48} \mathrm{H}_{58} \mathrm{Ni}_{4} \mathrm{~N}_{8} \mathrm{O}_{13}$ (1189.8): C-48.45, H4.91, N-9.42; found: C-48.05, H-4.71, N-9.57. Block shaped green crystals also obtained in alternative way, by slow evaporation of the resulting solution in acetone, but that was not suitable for X-ray diffraction. 
Synthesis of $\left[\mathbf{C o}^{\mathrm{II}}{ }_{4}(\mathbf{M e O H})_{\mathbf{3}}\left(\mathbf{H}_{\mathbf{2}} \mathbf{O}\right)\left(\mathbf{L}^{\mathbf{9}}\right)_{\mathbf{4}}\right] \cdot \mathbf{C H}_{\mathbf{2}} \mathbf{C l}_{\mathbf{2}} \quad(\mathbf{1 2}): \quad \mathrm{Co}\left(\mathrm{ClO}_{4}\right)_{2} \cdot 6 \mathrm{H}_{2} \mathrm{O} \quad\left(\begin{array}{llll}180 & \mathrm{mg}, & 0.49\end{array}\right.$ mmol) was added to a solution of $\mathbf{H}_{2} \mathbf{L}^{9}(100 \mathrm{mg}, 0.49 \mathrm{mmol})$ in $15 \mathrm{ml}$ methanol under $\mathrm{N}_{2^{-}}$ atmosphere, and then $\mathrm{NaOH}(40 \mathrm{mg}, 0.98 \mathrm{mmol})$ in $5 \mathrm{ml}$ methanol was added drop wise to the reaction mixture, affording a reddish brown precipitate immediately. The brown precipitate was collected by filtration, washed with methanol, and then dried in air. It was recrystallised by layering a solution of $\mathrm{CH}_{2} \mathrm{Cl}_{2}$ with $n$-hexane. Yield: $25 \mathrm{mg}$ (17\%). MS (ESI, $\left.\mathrm{CH}_{2} \mathrm{Cl}_{2}\right): m / z(\%), 1046.0(98)\left[\left(\mathrm{L}^{9}\right)_{4} \mathrm{Co}_{4}+\mathrm{H}\right]^{+}$. IR (KBr): $\tilde{v} 3570(\mathrm{~s}), 3410(\mathrm{br}), 3115$ (w), 3045 (w), 2920 (w), 2863 (w), 1596 (s), 1555 (s), 1515 (m), 1498 (s), 1464 (s), 1439 (m), 1413 (w), 1350 (w), 1313 (s), 1254 (m), 1226 (w), 1201 (w), 1190 (w), 1130 (s), 1087 (m), 1065 (m), $951(\mathrm{w}), 881(\mathrm{~m}), 848(\mathrm{~m}), 767(\mathrm{~m}), 688(\mathrm{~m}), 614(\mathrm{w}) \mathrm{cm}^{-1}$. Elemental analysis (\%) calculated for $\mathrm{C}_{48} \mathrm{H}_{56} \mathrm{Cl}_{2} \mathrm{Co}_{4} \mathrm{~N}_{8} \mathrm{O}_{12}$ (1243.63): C-46.36, H-4.54, N-9.01; found: C-47.78, H-4.55, N-9.53.

Synthesis of $\mathbf{F e}^{\mathrm{II}}{ }_{\mathbf{4}}(\mathbf{M e O H})_{\mathbf{4}}\left(\mathbf{L}^{\mathbf{9}}\right)_{\mathbf{4}}(\mathbf{1 3}): \mathrm{FeCl}_{2} \cdot 4 \mathrm{H}_{2} \mathrm{O}(98 \mathrm{mg}, 0.49 \mathrm{mmol})$ was added to a solution of $\mathbf{H}_{2} \mathbf{L}^{\mathbf{9}}$ (100 mg, $\left.0.49 \mathrm{mmol}\right)$ in $20 \mathrm{ml}$ methanol under $\mathrm{N}_{2}$-atmosphere, and then $\mathrm{NEt}_{3}$ (100 mg, $0.98 \mathrm{mmol}$ ) in $2 \mathrm{ml}$ methanol was added drop wise to the reaction mixture, affording a yellowish green precipitate immediately. The yellowish green precipitate was collected by filtration, washed with methanol, and then dried in vacuum. The resulting greenish yellow powder was dissolved in $\mathrm{CH}_{2} \mathrm{Cl}_{2}(20 \mathrm{ml})$, layered with $n$-hexane under inert atmosphere, green crystals of $\mathbf{1 3}$ were separated after several days. Yield: $15 \mathrm{mg}$ (11\%). IR (KBr): ขึ 3107 (w), 2962 (w), 2907 (w), 2857 (w), 2806 (w), 2610 (w), 1597 (s), 1558 (s), 1514 (s), 1485 (m), 1466 (s), 1439 (m), 1414 (w), 1349 (w), 1310 (s), 1259 (m), 1225 (w), 1198 (w), 1170 (w), 1130 (s), 1084 (w), 1061 (w), 1028 (m), 957 (w), 878 (m), 849 (m), 801 (w), $769(\mathrm{w}), 744(\mathrm{~m}), 686(\mathrm{~m}), 612(\mathrm{w}) \mathrm{cm}^{-1}$. Elemental analysis (\%) calculated for $\mathrm{C}_{48} \mathrm{H}_{56} \mathrm{Fe}_{4} \mathrm{~N}_{8} \mathrm{O}_{12}$ (1160.41): C-49.68, H-4.86, N-9.66; found: C-49.78, H-4.80, N-9.53.

Synthesis of $\left[\mathrm{Mn}^{\mathrm{III}}{ }_{2} \mathrm{Ni}^{\mathrm{II}}{ }_{3}\left(\mathrm{~L}^{9}\right)_{4}\left(\mathrm{HL}^{9}\right)_{2} \mathrm{Cl}_{2}\left(\mathrm{H}_{2} \mathrm{O}\right)_{2}\right] \cdot 8 \mathrm{CH}_{2} \mathrm{Cl}_{2}\left(14 \cdot 8 \mathrm{CH}_{2} \mathrm{Cl}_{2}\right)$ : A solution of $\mathrm{NEt}_{3}$ (23 mg, $0.22 \mathrm{mmol}, 1.5$ equiv) in acetonitrile $(3 \mathrm{ml})$ was added dropwise to a solution of $\mathrm{NiCl}_{2} \cdot 6 \mathrm{H}_{2} \mathrm{O}$ (26 mg, $0.110 \mathrm{mmol}, 0.75$ equiv) and $\mathbf{H}_{2} \mathbf{L}^{9}$ (30mg, $0.147 \mathrm{mmol}, 1$ equiv) in acetonitrile $(15 \mathrm{ml})$ at room temperature. The resultant green solution was then added dropwise to a brown solution of $\mathrm{MnCl}_{2} \cdot 4 \mathrm{H}_{2} \mathrm{O}$ (29 $\mathrm{mg}, 0.147 \mathrm{mmol}, 1$ equiv), $\mathbf{H}_{2} \mathbf{L}^{\mathbf{9}}$ (30 mg, $0.147 \mathrm{mmol}, 1$ equiv), and $\mathrm{NEt}_{3}(23 \mathrm{mg}, 0.22 \mathrm{mmol}, 1.5$ equiv) in acetonitrile ( $15 \mathrm{ml})$, and the reaction mixture was stirred for $8 \mathrm{~h}$ at room temperature. Evaporation of all volatile material under reduced pressure gave a brown solid. This crude product was dissolved in 
dichloromethane $(25 \mathrm{ml})$, and plate-shaped brown crystals of $14 \cdot 8 \mathrm{CH}_{2} \mathrm{Cl}_{2}$ were obtained by slow evaporation of the solution. Due to the loss of solvent molecules the crystals crumble after separation from the mother liquor. Yield: $40 \mathrm{mg}$ (48\%). IR (KBr): ṽ 3582 (w), 3458 (br), 3131 (w), 3111 (w), 3052 (w), 2928 (m), 2841 (m), 1627 (m), 1598 (s), 1563 (m), 1502 (w), 1506 (s), 1470 (s), 1443 (s), 1412 (m), 1357 (w), 1308 (s), 1256 (w), 1234 (w), 1204 (w), 1132 (s), 1084 (s), 948 (w), 857 (m), 850 (w), 805 (w), 766 (m), 743 (w), 701 (m), 642 (w), $609(\mathrm{~m}) \mathrm{cm}^{-1}$. Elemental analysis $(\%)$ calculated for $\mathrm{C}_{67.5} \mathrm{H}_{69} \mathrm{~N}_{12} \mathrm{O}_{14} \mathrm{Mn}_{2} \mathrm{Ni}_{3} \mathrm{Cl}_{5}\left(\mathbf{1 4} \cdot 1.5 \mathrm{CH}_{2} \mathrm{Cl}_{2}\right.$; 1735.6): C-46.71, H-4.01, N-9.68; found: C-46.46, H-4.18, N-9.67.

Synthesis of $\left[\mathrm{Mn}^{\mathrm{III}}{ }_{2} \mathrm{Ni}^{\mathrm{II}}{ }_{3}\left(\mathrm{~L}^{9}\right)_{4}\left(\mathrm{HL}^{9}\right)_{2} \mathrm{Cl}_{2}\left(\mathrm{H}_{2} \mathrm{O}\right)_{2}\right] \cdot 6 \mathrm{C}_{2} \mathrm{H}_{4} \mathrm{Cl}_{2}\left(14 \cdot 6 \mathrm{C}_{2} \mathrm{H}_{4} \mathrm{Cl}_{2}\right)$ : It was synthesized in similar way as like compound $\mathbf{1 4} \cdot 8 \mathrm{CH}_{2} \mathrm{Cl}_{2}$, here the crude product was recrystallised from 1,2-dichloroethane instead of dichloromethane, brown crystals of $14 \cdot 6 \mathrm{C}_{2} \mathrm{H}_{4} \mathrm{Cl}_{2}$ were obtained by slow evaporation of the resulting solution. The crystals crumble after separation from the mother liquor, due to loss of solvent molecules. Yield: 50\%. IR (KBr): ṽ 3585 (w), 3433 (br), 3134 (w), 3106 (w), 3053 (w), 2924 (m), 2846 (m), 1627 (m), 1598 (s), 1563 (s), 1501 (w), 1506 (s), 1470 (s), 1443 (s), 1412 (m), 1358 (w), 1306 (s), 1258 (w), 1232 (w), 1200 (w), 1156 (w), 1132 (s), 1082 (s), 1050 (m), 1038 (w), 947 (m), 871 (w), 856 (m), 841 (m), 790 (w), $772(\mathrm{~m}), 755(\mathrm{w}), 746(\mathrm{~m}), 702(\mathrm{~m}), 664(\mathrm{w}), 653(\mathrm{w}), 643(\mathrm{~m}), 612(\mathrm{w}) \mathrm{cm}^{-1}$. Elemental analysis (\%) calculated for $\mathrm{C}_{72} \mathrm{H}_{78} \mathrm{~N}_{12} \mathrm{O}_{14} \mathrm{Mn}_{2} \mathrm{Ni}_{3} \mathrm{Cl}_{8}\left(\mathbf{1 4} \cdot 3 \mathrm{C}_{2} \mathrm{H}_{4} \mathrm{Cl}_{2}\right.$, 1905.0): C-45.39, H-4.13, N-8.82; found: C-45.20, H-4.23, N-9.12.

Synthesis of $\left[\mathrm{Mn}^{\mathrm{III}}{ }_{2} \mathrm{Ni}^{\mathrm{II}}{ }_{3}\left(\mathrm{~L}^{9}\right)_{4}\left(\mathrm{HL}^{9}\right)_{2} \mathrm{Br}_{2}\left(\mathrm{H}_{2} \mathrm{O}\right)_{2}\right] \cdot 8 \mathrm{CH}_{2} \mathrm{Cl}_{2}\left(15 \cdot 8 \mathrm{CH}_{2} \mathrm{Cl}_{2}\right)$ : Compound 15 was obtained in similar manner as described for 14, but using $\mathrm{MnBr}_{2} \cdot 4 \mathrm{H}_{2} \mathrm{O}$ instead of $\mathrm{MnCl}_{2} \cdot 4 \mathrm{H}_{2} \mathrm{O}$. Brown crystals of $\mathbf{1 5} \cdot 8 \mathrm{CH}_{2} \mathrm{Cl}_{2}$ were obtained by slow evaporation of a solution of the crude product in dichlormethane. Yield: $50 \mathrm{mg}$ (54\%). IR (KBr): ṽ 3588 (w), 3437 (br), 3113 (w), 3052 (w), 2928 (m), 2842 (m), 2363 (w), 1629 (w), 1598 (s), 1561 (m), 1507 (s), 1469 (s), 1443 (s), 1412 (m), 1361 (m), 1325 (w), 1306 (s), 1269 (w), 1256 (w), 1233 (w), 1202 (w), 1156 (w), 1134 (s), 1084 (s), 1050 (w), 948 (w), 856 (m), 841 (w), 769 (w), 756 (m), $742(\mathrm{w}), 700(\mathrm{~m}), 664(\mathrm{w}), 635(\mathrm{w}), 638(\mathrm{w}), 610(\mathrm{~m}) \mathrm{cm}^{-1}$. Elemental analysis (\%) calculated for $\mathrm{C}_{66.5} \mathrm{H}_{67} \mathrm{~N}_{12} \mathrm{O}_{14} \mathrm{Mn}_{2} \mathrm{Ni}_{3} \mathrm{ClBr}_{2}\left(\mathbf{1 6} \cdot 0.5 \mathrm{CH}_{2} \mathrm{Cl}_{2}\right.$; 1731.5): C-45.92, H-3.88, N-9.66; found: C-46.29, H-4.16, N-9.15.

Synthesis of $\left[\mathrm{Mn}^{\mathrm{III}}{ }_{2} \mathrm{Mn}^{\mathrm{II}}\left(\mathrm{L}^{9}\right)_{2}(\mathrm{OAc})_{2}(\mathrm{OMe})_{2}(\mathrm{MeOH})_{2}\right] \cdot \mathrm{MeOH},(16) . \mathrm{Mn}(\mathrm{OAc})_{2} \cdot 4 \mathrm{H}_{2} \mathrm{O}(90$ $\mathrm{mg}, 0.36 \mathrm{mmol})$ was added to a solution of $\mathbf{H}_{\mathbf{2}} \mathbf{L}^{\mathbf{9}}(50 \mathrm{mg}, 0.2448 \mathrm{mmol})$ in $20 \mathrm{ml}$ methanol and then ${ }^{n} \mathrm{Bu}_{4} \mathrm{NOH}(127 \mathrm{mg}, 0.4896 \mathrm{mmol})$ in $5 \mathrm{ml}$ methanol was added drop wise to the 
reaction mixture, turned deep brown solution by air oxidation, and then without any stir reaction mixture was kept at room temperature, after few days dark red crystals were obtained by slow evaporation of the resulting solution. Yield: $15 \mathrm{mg}$ (11\%). IR (KBr): v 3419 (br), 2924 (w), 2858 (w), 2770 (w), 2358 (w), 2346 (w), 1601 (w), 1558 (s), 1516 (m), 1471 (s), 1446 (m), 1416 (s), 1363 (w), 1310 (s), 1252 (m), 1204 (w), 1134 (m), 1093 (m), 1035 (w), 1070 (s), 1035 (w), 897 (m), 858 (m), 757 (m), $701(\mathrm{w}), 656(\mathrm{w}), 640(\mathrm{w}) \mathrm{cm}^{-1}$. Elemental analysis (\%) calculated for $\mathrm{C}_{31} \mathrm{H}_{44} \mathrm{Mn}_{3} \mathrm{~N}_{4} \mathrm{O}_{13}$ (1189.8): C-44.04, H-5.25, N-6.63; found: C43.60, H-4.91, N-6.42.

Synthesis of $\left[\mathrm{Mn}^{\mathrm{III}}{ }_{4} \mathrm{Mn}^{\mathrm{II}}{ }_{2}\left(\mathrm{~L}^{\mathbf{9}}\right)_{\mathbf{4}} \mathrm{Cl}_{4}\left(\mathrm{H}_{2} \mathrm{O}\right)_{4}\left(\boldsymbol{\mu}_{4}-\mathrm{O}\right)_{2}\right] \cdot \mathbf{4 C H}_{3} \mathrm{CN}$, 17: A solution of $\mathrm{NEt}_{3}(25 \mathrm{mg}$, 0.2448 mmol, 2 equiv) in acetonitrile $(3 \mathrm{ml})$ was added drop wise to a solution of $\mathrm{MnCl}_{2} \cdot 4 \mathrm{H}_{2} \mathrm{O}$ (25 mg, $0.1224 \mathrm{mmol}, 1$ equiv) and $\mathbf{H}_{2} \mathbf{L}^{9}$ (25 mg, $0.1224 \mathrm{mmol}, 1$ equiv) in acetonitrile $(25 \mathrm{ml})$ at room temperature, the solution turned deep brown by air oxidation, and stirred for 10 mints. The resulting solution was bubbled with $\mathrm{N}_{2}$ for few mints, and then it was added drop wise to a colourless solution of $\mathrm{Mn}\left(\mathrm{ClO}_{4}\right)_{2} \cdot 6 \mathrm{H}_{2} \mathrm{O}$ (44 mg, $.092 \mathrm{mmol}, 0.75$ equiv), $\mathbf{H}_{2} \mathbf{L}^{9}$ (25 mg, $0.1224 \mathrm{mmol}, 1$ equiv), and $\mathrm{NEt}_{3}$ (19 mg, $0.1836 \mathrm{mmol}, 1.5$ equiv) in deoxygenated acetonitrile $(25 \mathrm{ml})$ under nitrogen atmosphere, and the reaction mixture was stirred for 2 mints at room temperature, and then resulting deep brown solution was exposed in air, block shaped dark red crystals were obtained by slow evaporation of the resulting solution. Yield: 15 mg (16\%). IR (KBr): ṽ 3376 (br), 3136 (w), 2927 (w), 2855 (w), 2360 (s), 2358 (m), 1601 (s), 1567 (m), 1512 (s), 1470 (s), 1445 (m), 1418 (m), 1362 (m), 1308 (s), 1244 (m), 1205 (w), 1136 (m), 1092 (m), 1072 (s), 1041 (w), 891 (w), 856 (m), 784 (w), 757 (m), $701 \quad(\mathrm{~m}), 655(\mathrm{w}), 604 \quad(\mathrm{w}) \mathrm{cm}^{-1}$. Elemental analysis (\%) calculated for $\mathrm{C}_{52} \mathrm{H}_{60} \mathrm{Cl}_{4} \mathrm{Mn}_{6} \mathrm{~N}_{12} \mathrm{O}_{14}$ (1548.56): C-40.33, H-3.91, N-10.85; found: C-40.07, H-3.17, N-9.93.

\section{Preparation of the Tetranuclear Metal Complexes $\left[\mathrm{NHEt}_{3}\right]\left[\left(\mathrm{L}^{9}\right)_{2} \mathrm{Mn}^{\mathrm{III}}{ }_{2}\left\{\left(\mathrm{NO}_{3}\right)_{2} \mathrm{Ln}^{\mathrm{III}}\right\}_{2}\right.$} $\left.\left(\mathrm{O}_{2} \mathrm{CPh}\right)_{3}\left(\mu_{4}-\mathrm{O}\right)\right] \cdot \mathbf{x C H} \mathrm{CH}_{3} \mathbf{C N} \cdot \mathbf{y} \mathrm{CH}_{2} \mathrm{Cl}_{2}$ 18-20: The general procedure for the preparation of the metal complexes 18-20 is as follows. $\mathrm{Mn}\left(\mathrm{O}_{2} \mathrm{CPh}\right)_{2} \cdot 2 \mathrm{H}_{2} \mathrm{O}(41 \mathrm{mg}, 0.1224 \mathrm{mmol})$ was added to a solution of $\mathbf{H}_{2} \mathbf{L}^{9}$ (25 mg, $0.1224 \mathrm{mmol}$ ) in $20 \mathrm{ml} \mathrm{CH}{ }_{3} \mathrm{CN}$ and then $\mathrm{NEt}_{3}$ (25 mg, 0.2448 mmol) in $2 \mathrm{ml} \mathrm{CH}{ }_{3} \mathrm{CN}$ was added drop wise to the reaction mixture, the solution become turned deep brown by air oxidation, and then solid $\mathrm{Ln}\left(\mathrm{NO}_{3}\right)_{2} \cdot 6 \mathrm{H}_{2} \mathrm{O}(55 \mathrm{mg}, 0.1224 \mathrm{mmol})$ $\left[\mathrm{Ln}=\mathrm{Gd}^{\mathrm{III}}(\mathbf{1 8}), \mathrm{Tb}^{\mathrm{III}}(\mathbf{1 9}), \mathrm{Dy}^{\mathrm{III}}(\mathbf{2 0})\right]$ was added to the reaction mixture, and stirred for 12 hrs at room temperature. Evaporation of all volatile material under reduced pressure gave a green solid. This crude product was dissolved in dichloromethane $(25 \mathrm{ml})$, and plate-shaped 
brown crystals were obtained by slow evaporation of the solution. The characterisation data for these complexes are given below.

$\left[\mathrm{NHEt}_{3}\right]\left[\left(\mathrm{L}^{9}\right)_{2} \mathrm{Mn}^{\mathrm{III}}{ }_{2}\left\{\left(\mathrm{NO}_{3}\right)_{2} \mathrm{Gd}^{\mathrm{III}}\right\}_{2}\left(\mathrm{O}_{2} \mathrm{CPh}\right)_{3}\left(\mu_{4}-\mathrm{O}\right)\right] \cdot \mathbf{x C H}_{3} \mathrm{CN} \cdot \mathbf{y C H}_{2} \mathrm{Cl}_{2}(18)$. Yield: $50 \mathrm{mg}$ (52\%). IR (KBr): ขึ 3433 (br), 3125 (w), 3064 (w), 2936 (w), 2868 (s), 1601 (s), 1539 (w), 1492 (w), 1471 (w), 1445 (m), 1401 (s), 1384 (m), 1307 (s), 1236 (m), 1209 (w), 1175 (m), 1138 (w), 1091 (w), 1067 (m), 1028 (m), 893 (w), 852 (m), 816 (w), 762 (w), 723 (s), 688 $(\mathrm{w}), 664(\mathrm{~m}), 624(\mathrm{~m}) \mathrm{cm}^{-1}$. Elemental analysis (\%) calculated for $\mathrm{C}_{49} \mathrm{H}_{51} \mathrm{Gd}_{2} \mathrm{Mn}_{2} \mathrm{~N}_{9} \mathrm{O}_{23}$ (1558.4): C-37.77, H-3.30, N-8.09; found: C-37.07, H-3.17, N-7.63

[NHEt $\left.{ }_{3}\right]\left[\left(\mathrm{L}^{9}\right)_{2} \mathrm{Mn}^{\mathrm{III}}{ }_{2}\left\{\left(\mathrm{NO}_{3}\right)_{2} \mathrm{~Tb}^{\mathrm{III}}\right\}_{2}\left(\mathrm{O}_{2} \mathrm{CPh}\right)_{3}\left(\mu_{4}-\mathrm{O}\right)\right] \cdot \mathbf{x C H}_{3} \mathrm{CN} \cdot \mathbf{y C H}_{2} \mathrm{Cl}_{2}(19)$. Yield: $40 \mathrm{mg}$ (42\%). IR (KBr): ข̃ 3397 (br), 3142 (w), 3060 (w), 2931 (w), 2865 (s), 1595 (s), 1547 (s), 1511 (m), 1472 (w), 1445 (m), 1404 (s), 1364 (w), 1309 (s), 1251 (m), 1239 (w), 1204 (w), 1175 (m), 1135 (m), 1088 (w), 1067 (m), 1043 (w), 1024 (w), 895 (m), 856 (m), 817 (w), 781 (w), 753 (s), 721 (s), 701 (s), 689 (w), 675 (m), 628 (w) $\mathrm{cm}^{-1}$. Elemental analysis (\%) calculated for $\mathrm{C}_{49} \mathrm{H}_{51} \mathrm{~Tb}_{2} \mathrm{Mn}_{2} \mathrm{~N}_{9} \mathrm{O}_{23}$ (1561.7): C-37.68, H-3.29, N-8.07; found: C-37.27, H3.17, N-7.83.

$\left[\mathrm{NHEt}_{3}\right]\left[\left(\mathrm{L}^{9}\right)_{2} \mathrm{Mn}^{\mathrm{III}}{ }_{2}\left\{\left(\mathrm{NO}_{3}\right)_{2} \mathrm{Dy}{ }^{\mathrm{III}}\right\}_{2}\left(\mathrm{O}_{2} \mathrm{CPh}\right)_{3}\left(\mu_{4}-\mathrm{O}\right)\right] \cdot \mathbf{x C H}_{3} \mathrm{CN} \cdot \mathbf{y C H}_{2} \mathrm{Cl}_{2}(20)$. Yield: $38 \mathrm{mg}$ (39\%). IR (KBr): ขี 3429 (br), 3123 (w), 3054 (w), 2931 (w), 2848 (s), 1601 (s), 1541 (m), 1508 (w), 1492 (w), 1471 (m), 1451 (m), 1402 (s), 1374 (w), 1311 (s), 1236 (m), 1210 (w), 1175 (m), 1136 (w), 1089 (w), 1067 (m), 1029 (m), 895 (w), 852 (m), $815(\mathrm{w}), 759$ (w), 723 (s), $688(\mathrm{w}), \quad 666(\mathrm{~m}), 627 \quad(\mathrm{~m}) \quad \mathrm{cm}^{-1}$. Elemental analysis (\%) calculated for $\mathrm{C}_{49} \mathrm{H}_{51} \mathrm{Dy}_{2} \mathrm{Mn}_{2} \mathrm{~N}_{9} \mathrm{O}_{23}$ (1568.8): C-37.51, H-3.28, N-8.04; found: C-37.17, H-3.20, N-7.73.

Synthesis of $\left[\mathrm{L}^{12} \mathrm{Cu}_{2}{ }_{2}^{\mathrm{II}}(\boldsymbol{\mu}-\mathrm{MeOH} \cdots \mathrm{OH})\right] \cdot 2.5 \mathrm{MeOH}(\mathbf{2 1})$ : A solution of $\mathrm{Cu}\left(\mathrm{BF}_{4}\right)_{2}, 4 \mathrm{H}_{2} \mathrm{O}(110$ $\mathrm{mg}, 0.36 \mathrm{mmol})$ in methanol $(20 \mathrm{ml})$ was added to a stirred solution of $\mathbf{H}_{\mathbf{3}} \mathbf{L}^{\mathbf{1 2}}(100 \mathrm{mg}, 0.18$ $\mathrm{mmol})$ in methanol $(50 \mathrm{ml})$ resulting in a green solution. A solution of $\mathrm{NaOMe}(29 \mathrm{mg}, 0.54$ mmol) in methanol $(5 \mathrm{ml})$ was added dropwise at room temperature, stirred overnight, and then filtered. Needle shaped dark green crystals of $\mathbf{2 1}$ were separated using slow evaporation of the resulting solution. Yield: $44 \mathrm{mg}(30 \%)$. MS (ESI, MeOH): $\mathrm{m} / \mathrm{z}(\%)=682.9$ (52) $\left[\mathrm{L}^{12} \mathrm{Cu}_{2}\right]^{+}$. IR (KBr): $\widetilde{v} 3650$ (w), 3434 (br), 2954 (s), 2904 (w), 2879 (w), 1929 (w), 1624 (s), 1532 (m), 14460 (w), 1433 (m), 1389 (m), 1358 (w), 1326 (w), 1281 (w), 1255 (m), 1241 (w), $1199(\mathrm{w}), 1167$ (m), $1061(\mathrm{w}), 914(\mathrm{w}), 875(\mathrm{w}), 840(\mathrm{~m}), 781(\mathrm{w}), 728(\mathrm{w}), 650(\mathrm{w}) \mathrm{cm}^{-1}$. 
Elemental analysis (\%) calculated for $\mathrm{C}_{38.5} \mathrm{H}_{61} \mathrm{Cu}_{2} \mathrm{~N}_{4} \mathrm{O}_{6.5}$ (811.0): C-57.02, H-7.58, N-6.91; found: C-56.53, H-6.77, N-7.63.

Synthesis of $\left[\mathbf{L}^{12} \mathbf{C u}^{\text {II }}{ }_{2}(\boldsymbol{\mu}\right.$-OAc) $] \cdot \mathbf{1 . 3 3} \mathbf{C H C l}_{3}(\mathbf{2 2}): 2$ equivalent of $\mathrm{Cu}(\mathrm{OAc})_{2} \cdot \mathrm{H}_{2} \mathrm{O}(72 \mathrm{mg}$, $0.36 \mathbf{m m o l})$ in methanol $(10 \mathrm{ml})$ was added to a solution of $\mathbf{H}_{\mathbf{3}} \mathbf{L}^{\mathbf{1 2}}(100 \mathrm{mg}, 0.18 \mathrm{mmol})$ in methanol $(30 \mathrm{ml})$, stirred overnight, during which a green precipitate formed. This was filtered off, washed with methanol, dried in air. The resulting green powder was dissolved in chloroform $(10 \mathrm{ml})$, and then layered with $n$-hexane; green crystals of 22 were separated after several days. Yield: $30 \mathrm{mg}(19 \%)$. MS (ESI, $\left.\mathrm{CHCl}_{3}\right): \mathrm{m} / z(\%)=683.2(45)\left[\mathrm{L}^{12} \mathrm{Cu}_{2}\right]^{+}$. IR (KBr): ข̃ 2954 (s), 2905 (w), 2870 (w), 1621 (s), 1572 (m), 1529 (m), 1458 (w), 1431 (m),

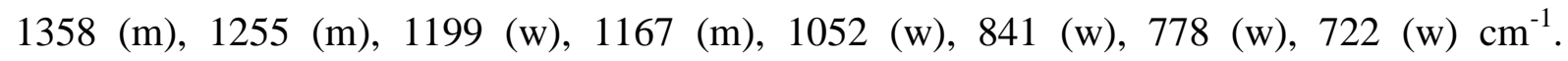
Elemental analysis (\%) calculated for $\mathrm{C}_{37.33} \mathrm{H}_{50.33} \mathrm{Cl}_{0.99} \mathrm{Cu}_{2} \mathrm{~N}_{4} \mathrm{O}_{4}(781.3)$ : C-57.39, H-6.49, N7.17; found: C-57.97, H-7.05, N-7.63.

Synthesis of $\left[\mathrm{L}^{12} \mathrm{Ni}^{\mathrm{II}}{ }_{2}(\mu-\mathrm{OAc})\right] \cdot \mathbf{1 . 3 3 C H C l}_{3}(\mathbf{2 3})$ : It was obtained in similar manner as described for 22, but using $\mathrm{Ni}(\mathrm{OAc})_{2} \cdot 4 \mathrm{H}_{2} \mathrm{O}$ used instead of $\mathrm{Cu}(\mathrm{OAc})_{2} \cdot \mathrm{H}_{2} \mathrm{O}$. Yield: $25 \mathrm{mg}$ (16\%). IR (KBr): ข̃ 2954 (s), 2906 (w), 2871 (w), 1615 (s), 1590 (m), 1531 (m), 1451 (w), 1435 (m), 1329 (m), 1256 (m), 1202 (w), 1172 (w), 1160 (m), 1026 (w), 929 (w), 754 (w) cm 1. Elemental analysis (\%) calculated for $\mathrm{C}_{37} \mathrm{H}_{50} \mathrm{Ni}_{2} \mathrm{~N}_{4} \mathrm{O}_{4}(732.2)$ : C-60.69, H-6.88, N-7.65; found: C-60.57, H-7.05, N-7.63.

Synthesis of $\left[\mathbf{Z n}_{\mathbf{4}}\left(\mathbf{L}^{\mathbf{1 2}}\right)_{\mathbf{2}}\left(\mathbf{H}_{\mathbf{2}} \mathbf{L}^{\mathbf{1 2}}\right)_{\mathbf{2}}\right]$ (24): 2 equivalent of $\mathrm{Zn}\left(\mathrm{ClO}_{4}\right)_{2} \cdot 6 \mathrm{H}_{2} \mathrm{O}$ (134 mg, $\left.0.36 \mathrm{mmol}\right)$ in methanol (5 ml) was added drop wise to a stirred solution of $\mathbf{H}_{\mathbf{3}} \mathbf{L}^{\mathbf{1 2}}$ (100 $\left.\mathrm{mg}, 0.18 \mathrm{mmol}\right)$ in methanol $(30 \mathrm{ml})$ resulting in a yellow solution. A solution of $\mathrm{PhCO}_{2} \mathrm{Na}(78 \mathrm{mg}, 0.54 \mathrm{mmol})$, in methanol (20 ml) was added drowise, stirred overnight, yellow precipitate was collected by filtration, washed with methanol, dried in air. The resulting yellow powder was dissolved in (1:1) dichloromethane-hexane $(20 \mathrm{ml})$ yellow crystals were obtained by slow evaporation of the resulting solution. Yield: $40 \mathrm{mg}(36 \%)$. MS (MALDI, dithranol): $\mathrm{m} / \mathrm{z}(\%)=2488.3(50)$ $\left[\mathrm{Zn}_{4}\left(\mathrm{~L}^{12}\right)_{2}\left(\mathrm{H}_{2} \mathrm{~L}^{12}\right)_{2}+\mathrm{H}^{+}\right], 1930.3(100)\left[\mathrm{Zn}_{4}\left(\mathrm{~L}^{12}\right)_{2}\left(\mathrm{H}_{2} \mathrm{~L}^{12}\right)\right]^{+}, 1305.7(53)\left[\mathrm{Zn}_{3}\left(\mathrm{~L}^{12}\right)_{2}-\mathrm{H}^{+}\right], 1242.6$ (28) $\left[\mathrm{Zn}_{2}\left(\mathrm{~L}^{12}\right)_{2}+\mathrm{H}^{+}\right], 687.2(10)\left[\mathrm{Zn}_{2} \mathrm{~L}^{12}\right]^{+}$. IR (KBr): $\widetilde{v} 3435(\mathrm{w}), 2956(\mathrm{~s}), 2908(\mathrm{w}), 2882$ (w), 1611 (s), 1532 (m), 14468 (m), 1437 (m), 1362 (m), 1320 (m), 1273 (w), 1254 (m), 1201 (w), $1164(\mathrm{~m}), 1135$ (w), 1047 (m), 914 (w), $874(\mathrm{w}), 831$ (m), $783(\mathrm{w}), 745(\mathrm{w}), 640(\mathrm{w})$ $\mathrm{cm}^{-1}$. Elemental analysis (\%) calculated for: $\mathrm{C}_{140} \mathrm{H}_{192} \mathrm{Zn}_{4} \mathrm{~N}_{16} \mathrm{O}_{8}(2487.3)$ : C-67.57, H-7.78, N9.01; found: C-66.62, H-7.88, N-8.98. ${ }^{1} \mathrm{H}$ NMR (500 MHz, $\left.\mathrm{CDCl}_{3}, 298 \mathrm{~K}\right): \delta(\mathrm{ppm})=1.21[\mathrm{~s}$, 
$\left.18 \mathrm{H} ; 3^{\prime}-\mathrm{C}\left(\mathrm{CH}_{3}\right)_{3}\right], 1.25\left[\mathrm{~s}, 18 \mathrm{H} ; 3-\mathrm{C}\left(\mathrm{CH}_{3}\right)_{3}\right], 1.32\left[\mathrm{~s}, 18 \mathrm{H} ; 5^{\prime}-\mathrm{C}\left(\mathrm{CH}_{3}\right)_{3}\right], 1.37$ [s, 18H; 5$\left.\mathrm{C}\left(\mathrm{CH}_{3}\right)_{3}\right], 4.27\left[\mathrm{~s}, 2 \mathrm{H} ; \mathrm{CH}_{2}\right], 4.41\left[\mathrm{~d}, 1 \mathrm{H} ; \mathrm{CH}_{2}\right], 4.55\left[\mathrm{~d}, 1 \mathrm{H} ; \mathrm{CH}_{2}\right], 5.77\left[\mathrm{~s}, 1 \mathrm{H} ; \mathrm{CH}^{\mathrm{pz}}\right], 6.25$ $\left[\mathrm{s}, 1 \mathrm{H} ; \mathrm{CH}^{\prime \mathrm{pz}}\right], 6.49$ [d, 1H; C2], 6.82 [d, 1H; C2'], 7.29 [d, 1H; C-4'], 7.41 [s, 1H; NH], 7.45 [d, 1H; C-4], 7.87 [s, 1H; NH'], 13.5 (br, 1H; $\left.\mathrm{OH}^{\prime}\right) .{ }^{13} \mathrm{C}\left\{{ }^{1} \mathrm{H}\right\} \mathrm{NMR}\left(125.75 \mathrm{MHz}, \mathrm{CDCl}_{3}, 298\right.$ $\mathrm{K}): \delta(\mathrm{ppm})=29.36,29.53,31.48,31.51,33.94,34.06,34.92,35.42,51.80,54.43,105.16$, $108.14,116.16,117.57,125.70,126.91,128.61,130.27,135.43,136.69,139.99,141.58$, $149.2,151.79,157.82,166.45,167.96,171.09$. 


\section{Chapter 10}

\section{Crystallography}

The crystal data and details of the data collections are collected in Tables 10.1 - 10.9. X-ray data were collected on a STOE IPDS II diffractometer (graphite monochromated Mo-K $\mathrm{K}_{\alpha}$ radiation, $\lambda=0.71073 \AA$ Á, $\omega$ scans) at $-140^{\circ} \mathrm{C}$. The structures were solved by direct methods and refined on $F^{2}$ using all reflections with SHELX-97 or SHELXL-97. ${ }^{108,109,110}$ 
Table 10.1. Crystal data and refinement details

\begin{tabular}{|c|c|c|c|}
\hline compound & 1 & $\mathbf{H}_{3} \mathbf{L}^{5}$ & 3 \\
\hline formula & $\mathrm{C}_{6} \mathrm{H}_{16} \mathrm{ClN}_{9}$ & $\mathrm{C}_{57} \mathrm{H}_{80} \mathrm{ClN}_{9} \mathrm{O}_{3}$ & $\mathrm{C}_{76} \mathrm{H}_{124} \mathrm{Cu}_{4} \mathrm{~N}_{14} \mathrm{O}_{14}$ \\
\hline $\mathrm{M}_{\mathrm{r}}$ & 249.73 & 974.75 & 1712.05 \\
\hline crystal size [mm] & $0.36 \times 0.18 \times 0.09$ & $0.39 \times 0.25 \times 0.12$ & $0.50 \times 0.23 \times 0.21$ \\
\hline crystal system & monoclinic & monoclinic & monoclinic \\
\hline space group & $P 2{ }_{1} / c$ & $P 2_{1} / n$ & $P 2_{1} / c$ \\
\hline$a[\AA]$ & $10.2759(7)$ & $18.3309(6)$ & $23.5629(4)$ \\
\hline$b[\AA]$ & $11.1481(5)$ & $10.2696(6)$ & $10.4317(2)$ \\
\hline$c[\AA]$ & $10.9016(8)$ & $29.9748(10)$ & $40.5566(7)$ \\
\hline$\alpha\left[^{\circ}\right]$ & 90 & 90 & 90 \\
\hline$\beta\left[^{\circ}\right]$ & $114.779(5)$ & $91.10(3)$ & $105.2240(10)$ \\
\hline$\gamma\left[^{\circ}\right]$ & 90 & 90 & 90 \\
\hline$V\left[\AA^{3}\right]$ & $1133.87(12)$ & $5641.7(4)$ & $9619.0(3)$ \\
\hline$Z$ & 4 & 4 & 4 \\
\hline$\rho_{\text {calcd. }}\left[\mathrm{g} \mathrm{cm}^{-3}\right]$ & 1.463 & 1.148 & 1.182 \\
\hline$F(000)$ & 528 & 2104 & 3624 \\
\hline$\mu\left[\mathrm{mm}^{-1}\right]$ & 0.329 & 0.118 & 0.932 \\
\hline$T_{\max } / T_{\min }$ & $0.9310 / 0.7377$ & $0.9335 / 0.7418$ & \\
\hline$h k l$ range & $\pm 13,+14,-12, \pm 13$ & $\pm 22, \pm 12,+31,-36$ & $+26,-27, \pm 12, \pm 47$ \\
\hline$\theta$ range $\left[{ }^{\circ}\right]$ & $2.18-27.00$ & $1.29-25.71$ & $1.18-24.61$ \\
\hline measured refl. & 10758 & 42350 & 58259 \\
\hline unique refl. $\left[R_{\text {int }}\right]$ & $2459(.0424)$ & $10658(.0822)$ & $15791(.0471)$ \\
\hline ref. param. / restraints & $176 / 0$ & $618 / 30$ & $1008 / 0$ \\
\hline goodness-of-fit & 1.035 & 1.059 & 1.040 \\
\hline$R 1, w R 2(I>2 \sigma(I))$ & $0.0373,0.0840$ & $0.1110,0.3005$ & $0.0539,0.1522$ \\
\hline$R 1, w R 2$ (all data) & $0.0517,0.0880$ & $0.1617,0.3231$ & $0.0647,0.1580$ \\
\hline resid. el. dens. $\left[\mathrm{e} \AA^{-3}\right]$ & $0.308 /-0.224$ & $0.678 /-0.453$ & $1.089 /-0.697$ \\
\hline
\end{tabular}


Table 10.2. Crystal data and refinement details

\begin{tabular}{|c|c|c|c|}
\hline compound & 4 & 5 & 6 \\
\hline formula & $\mathrm{C}_{148} \mathrm{H}_{212} \mathrm{Mn}_{4} \mathrm{~N}_{28} \mathrm{O}_{20}$ & $\mathrm{C}_{104} \mathrm{H}_{146} \mathrm{Fe}_{4} \mathrm{~N}_{16} \mathrm{O}_{17}$ & $\mathrm{C}_{24} \mathrm{H}_{26} \mathrm{MnN}_{14} \mathrm{O}_{7}$ \\
\hline $\mathrm{M}_{\mathrm{r}}$ & 2919.22 & 2115.77 & 677.53 \\
\hline crystal size $[\mathrm{mm}]$ & $0.43 \times 0.29 \times 0.19$ & $0.39 \times 0.29 \times 0.18$ & $0.50 \times 0.48 \times 0.43$ \\
\hline crystal system & monoclinic & othrorhombic & triclinic \\
\hline space group & $C 2 / c$ & $A b a 2$ & $P-1$ \\
\hline$a[\AA]$ & $36.9055(10)$ & $38.2483(11)$ & $7.6447(6)$ \\
\hline$b[\AA]$ & $18.7803(9)$ & $19.8080(9)$ & $14.338(10)$ \\
\hline$c[\AA]$ & $29.1657(9)$ & $17.4100(5)$ & $14.4515(10)$ \\
\hline$\alpha\left[^{\circ}\right]$ & 90 & 90 & $84.139(6)$ \\
\hline$\beta\left[^{\circ}\right]$ & $94.432(2)$ & 90 & $86.797(6)$ \\
\hline$\gamma\left[^{\circ}\right]$ & 90 & 90 & $82.866(6)$ \\
\hline$V\left[\AA^{3}\right]$ & $20154.2(13)$ & $13190.2(8)$ & $1562.2(2)$ \\
\hline$Z$ & 4 & 4 & 2 \\
\hline$\rho_{\text {calcd. }}\left[\mathrm{g} \mathrm{cm}^{-3}\right]$ & 0.963 & 1.065 & 1.44 \\
\hline$F(000)$ & 6224 & 4488 & 698 \\
\hline$\mu\left[\mathrm{mm}^{-1}\right]$ & 0.300 & 0.489 & 0.488 \\
\hline$h k l$ range & $\pm 43,+18,-21,+33,-34$ & $-44,+39, \pm 23,-18,+20$ & $\pm 9,+18,-16, \pm 18$ \\
\hline$\theta$ range $\left[{ }^{\circ}\right]$ & $1.11-24.59$ & $1.65-24.62$ & $1.42-26.79$ \\
\hline measured refl. & 41345 & 29287 & 19573 \\
\hline unique refl. [ $\left.R_{\mathrm{int}}\right]$ & $16543(.0942)$ & $10141(0.1268)$ & $6626[0.0327]$ \\
\hline ref. param. / restraints & $891 / 73$ & $638 / 1$ & $417 / 26$ \\
\hline goodness-of-fit & 1.010 & 1.041 & 1.024 \\
\hline$R 1, w R 2(I>2 \sigma(I))$ & $0.0774,0.1757$ & $0.1058,0.2652$ & $0.0811,0.2172$ \\
\hline$R 1, w R 2$ (all data) & $0.1320,0.1923$ & $0.1182,0.2741$ & $0.0916,0.2251$ \\
\hline resid. el. dens. [e $\left.\AA^{-3}\right]$ & $0.468 /-0.610$ & $1.142 /-0.600$ & $1.184 /-1.031$ \\
\hline
\end{tabular}


Table 10.3. Crystal data and refinement details

\begin{tabular}{|c|c|c|c|}
\hline compound & 7 & 8 & 9 \\
\hline formula & $\mathrm{C}_{48} \mathrm{H}_{48} \mathrm{Mn}_{2} \mathrm{~N}_{30} \mathrm{O}_{10}$ & $\mathrm{C}_{26} \mathrm{H}_{32} \mathrm{MnN}_{18} \mathrm{O}_{2}$ & $\mathrm{C}_{25} \mathrm{H}_{28} \mathrm{MnN}_{16} \mathrm{O}_{4}$ \\
\hline $\mathrm{M}_{\mathrm{r}}$ & 1315.04 & 683.64 & 651.57 \\
\hline crystal size [mm] & $0.24 \times 0.18 \times 0.06$ & $0.41 \times 0.22 \times 0.16$ & $0.50 \times 0.34 \times 0.13$ \\
\hline crystal system & triclinic & triclinic & triclinic \\
\hline space group & $P-1$ & $P-1$ & $P-1$ \\
\hline$a[\AA]$ & $14.2328(12)$ & $11.2636(5)$ & $6.7116(4)$ \\
\hline$b[\AA]$ & $14.4951(10)$ & $12.7851(6)$ & $14.4431(10)$ \\
\hline$c[\AA]$ & $15.2225(11)$ & $13.4006(6)$ & $15.0108(10)$ \\
\hline$\alpha\left[^{\circ}\right]$ & $93.583(6)$ & $115.026(3)$ & $92.697(6)$ \\
\hline$\beta\left[^{\circ}\right]$ & $103.199(6)$ & $108.553(4)$ & $98.606(5)$ \\
\hline$\gamma\left[{ }^{\circ}\right]$ & $94.415(6)$ & $99.712(4)$ & $92.980(5)$ \\
\hline$V\left[\AA^{3}\right]$ & $3032.4(4)$ & $1550.96(12)$ & $1434.55(16)$ \\
\hline$Z$ & 2 & 2 & 2 \\
\hline$\rho_{\text {calcd. }}\left[\mathrm{g} \mathrm{cm}^{-3}\right]$ & 1.440 & 1.464 & 1.555 \\
\hline$F(000)$ & 1352 & 710 & 694 \\
\hline$\mu\left[\mathrm{mm}^{-1}\right]$ & 0.497 & 0.484 & 0.525 \\
\hline$T_{\max } / T_{\min }$ & & $0.9460 / 0.8059$ & $0.9032 / 0.7314$ \\
\hline$h k l$ range & $-18,+14, \pm 18, \pm 19$ & $-14,+12, \pm 16,-16,+17$ & $-8,+7, \pm 18, \pm 18$ \\
\hline$\theta$ range $\left[{ }^{\circ}\right]$ & $1.77-27.06$ & $1.85-26.97$ & $1.37-26.78$ \\
\hline measured refl. & 25953 & 17581 & 16376 \\
\hline unique refl. $\left[R_{\text {int }}\right]$ & $12993[0.1641]$ & $6728[0.0657]$ & $6091[0.0499]$ \\
\hline ref. param. / restraints & $803 / 28$ & $423 / 3$ & $421 / 0$ \\
\hline goodness-of-fit & 1.009 & 1.041 & 1.041 \\
\hline$R 1, w R 2(I>2 \sigma(I))$ & $0.0922,0.2264$ & $0.0409,0.1066$ & $0.0531,0.1391$ \\
\hline$R 1, w R 2$ (all data) & $0.1458,0.2554$ & $0.0487,0.1108$ & $0.0705,0.1501$ \\
\hline resid. el. dens. $\left[\mathrm{e} \AA^{-3}\right]$ & $1.093 /-1.138$ & $0.640 /-0.724$ & $0.574 /-1.064$ \\
\hline
\end{tabular}


Table 10.4. Crystal data and refinement details

\begin{tabular}{|c|c|c|c|}
\hline compound & 10 & $\mathbf{H}_{2} \mathbf{L}^{9}$ & 11 \\
\hline formula & $\mathrm{C}_{26} \mathrm{H}_{26} \mathrm{MnN}_{16} \mathrm{O}_{4}$ & $\mathrm{C}_{11} \mathrm{H}_{22} \mathrm{O}_{2} \mathrm{~N}_{2}$ & $\mathrm{C}_{48} \mathrm{H}_{58} \mathrm{Ni}_{4} \mathrm{~N}_{8} \mathrm{O}_{13}$ \\
\hline $\mathrm{M}_{\mathrm{r}}$ & 681.57 & 204.23 & 1189.86 \\
\hline crystal size [mm] & $0.50 \times 0.28 \times 0.24$ & $0.50 \times 0.10 \times 0.10$ & $0.42 \times 0.21 \times 0.07$ \\
\hline crystal system & triclinic & tetragonal & tetragonal \\
\hline space group & $P-1$ & $I-4$ & $I 4_{1} / a$ \\
\hline$a[\AA]$ & $10.4827(4)$ & $18.723(3)$ & $16.8120(7)$ \\
\hline$b[\AA]$ & $12.6223(4)$ & $18.723(3)$ & $16.8120(7)$ \\
\hline$c[\AA]$ & $14.2137(5)$ & $5.708(12)$ & $17.5449(9)$ \\
\hline$\alpha\left[^{\circ}\right]$ & $77.851(3)$ & 90 & 90 \\
\hline$\beta\left[^{\circ}\right]$ & $72.139(3)$ & 90 & 90 \\
\hline$\gamma\left[{ }^{\circ}\right]$ & $69.838(3)$ & 90 & 90 \\
\hline$V\left[\AA^{3}\right]$ & $16668.78(10)$ & $2026.6(6)$ & 4958.9(4) \\
\hline$Z$ & 2 & 8 & 4 \\
\hline$\rho_{\text {calcd. }}\left[\mathrm{g} \mathrm{cm}^{-3}\right]$ & 1.356 & 1.339 & 1.594 \\
\hline$F(000)$ & 702 & 1920 & 2472 \\
\hline$\mu\left[\mathrm{mm}^{-1}\right]$ & 0.453 & 0.094 & 1.569 \\
\hline$T_{\max } / T_{\min }$ & & 0.9568 / 0.7731 & $0.8420 / 0.7030$ \\
\hline$h k l$ range & $-13,+12, \pm 15, \pm 17$ & $-22,+23, \pm 23,-6,+7$ & $\pm 21, \pm 21, \pm 22$ \\
\hline$\theta$ range $\left[{ }^{\circ}\right]$ & $1.52-26.73$ & $1.54-26.89$ & $1.68-27.15$ \\
\hline measured refl. & 21976 & 6161 & 44432 \\
\hline unique refl. [ $\left.R_{\mathrm{int}}\right]$ & $7065[0.0485]$ & $1207[0.1048]$ & $44432[0.000]$ \\
\hline ref. param. / restraints & $433 / 2$ & $144 / 0$ & $140 / 6$ \\
\hline goodness-of-fit & 1.064 & 1.046 & 1.043 \\
\hline$R 1, w R 2(I>2 \sigma(I))$ & $0.0430,0.1169$ & $0.0365,0.0905$ & $0.0707,0.1630$ \\
\hline$R 1, w R 2$ (all data) & $0.0466,0.1187$ & $0.0383,0.0916$ & $0.1268,0.1818$ \\
\hline resid. el. dens. [e $\left.\AA^{-3}\right]$ & $0.682 /-0.662$ & $0.160 /-0.169$ & $0.778 /-0.454$ \\
\hline
\end{tabular}


Table 10.5. Crystal data and refinement details

\begin{tabular}{|c|c|c|c|}
\hline compound & 12 & 13 & $14 \cdot 8 \mathrm{CH}_{2} \mathrm{Cl}_{2}$ \\
\hline formula & $\mathrm{C}_{48} \mathrm{H}_{56} \mathrm{Cl}_{2} \mathrm{Co}_{4} \mathrm{~N}_{8} \mathrm{O}_{12}$ & $\mathrm{C}_{48} \mathrm{H}_{56} \mathrm{Fe}_{4} \mathrm{~N}_{8} \mathrm{O}_{12}$ & $\mathrm{C}_{74} \mathrm{H}_{76} \mathrm{Cl}_{18} \mathrm{Mn}_{2} \mathrm{~N}_{12} \mathrm{Ni}_{3} \mathrm{O}_{14}$ \\
\hline $\mathrm{M}_{\mathrm{r}}$ & 1243.63 & 1160.41 & 2281.58 \\
\hline crystal size $[\mathrm{mm}]$ & $0.49 \times 0.29 \times 0.23$ & $0.50 \times 0.28 \times 0.26$ & $0.37 \times 0.26 \times 0.06$ \\
\hline crystal system & triclinic & tetragonal & triclinic \\
\hline space group & $P-1$ & $I 4_{1} / a$ & $P-1$ \\
\hline$a[\AA]$ & $14.1325(4)$ & $17.0749(7)$ & $13.3948(10)$ \\
\hline$b[\AA]$ & $14.4262(4)$ & $17.0749(7)$ & $13.8821(10)$ \\
\hline$c[\AA]$ & $14.9207(4)$ & $17.7535(8)$ & $14.6735(12)$ \\
\hline$\alpha\left[^{\circ}\right]$ & $114.765(2)$ & 90 & $90.078(6)$ \\
\hline$\beta\left[^{\circ}\right]$ & $110.576(2)$ & 90 & $115.446(6)$ \\
\hline$\gamma\left[^{\circ}\right]$ & $93.007(2)$ & 90 & $108.250(6)$ \\
\hline$V\left[\AA^{3}\right]$ & $2513.68(12)$ & $5176.1(4)$ & $2310.3(3)$ \\
\hline$Z$ & 2 & 4 & 1 \\
\hline$\rho_{\text {calcd. }}\left[\mathrm{g} \mathrm{cm}^{-3}\right]$ & 1.643 & 1.489 & 1.640 \\
\hline$F(000)$ & 1276 & 2400 & 1156 \\
\hline$\mu\left[\mathrm{mm}^{-1}\right]$ & 1.474 & 1.166 & 1.452 \\
\hline$T_{\max } / T_{\min }$ & $0.7297 / 0.5300$ & $0.6543 / 0.4527$ & 0.8624 / 0.5796 \\
\hline$h k l$ range & $\pm 17, \pm 18, \pm 17$ & $\pm 21, \pm 21, \pm 22$ & $\pm 16, \pm 16, \pm 17$ \\
\hline$\theta$ range $\left[{ }^{\circ}\right]$ & $1.58-26.74$ & $1.65-26.72^{\circ}$ & $1.56-25.70$ \\
\hline measured refl. & 33359 & 17300 & 25369 \\
\hline unique refl. $\left[R_{\text {int }}\right]$ & $10634[0.0360]$ & $2735[0.0696]$ & $8716[0.0802]$ \\
\hline ref. param. / restraints & $563 / 38$ & $627 / 58$ & $556 / 0$ \\
\hline goodness-of-fit & 1.046 & 1.016 & 1.122 \\
\hline$R 1, w R 2(I>2 \sigma(I))$ & $0.0312,0.0836$ & $0.0481,0.1100$ & $0.0905,0.2571$ \\
\hline$R 1, w R 2$ (all data) & $0.0364,0.0860$ & $0.0806,0.1211$ & $0.1116,0.2672$ \\
\hline resid. el. dens. [e $\left.\AA^{-3}\right]$ & $1.536 /-1.424$ & $0.379 /-0.342$ & $2.252 /-1.328$ \\
\hline
\end{tabular}


Table 10.6. Crystal data and refinement details

\begin{tabular}{|c|c|c|c|}
\hline compound & $14 \cdot 6 \mathrm{C}_{2} \mathrm{H}_{4} \mathrm{Cl}_{2}$ & $15 \cdot 8 \mathrm{CH}_{2} \mathrm{Cl}_{2}$ & 16 \\
\hline formula & $\begin{array}{l}\mathrm{C}_{78} \mathrm{H}_{90} \mathrm{Cl}_{14} \mathrm{Mn}_{2} \\
\mathrm{~N}_{12} \mathrm{Ni}_{3} \mathrm{O}_{14}\end{array}$ & $\begin{array}{l}\mathrm{C}_{74} \mathrm{H}_{82} \mathrm{Br}_{2} \mathrm{Cl}_{16} \mathrm{Mn}_{2} \\
\mathrm{~N}_{12} \mathrm{Ni}_{3} \mathrm{O}_{14}\end{array}$ & $\mathrm{C}_{31} \mathrm{H}_{44} \mathrm{Mn}_{3} \mathrm{~N}_{4} \mathrm{O}_{13}$ \\
\hline $\mathrm{M}_{\mathrm{r}}$ & 2201.93 & 2376.55 & 845.52 \\
\hline Crystal size [mm] & $0.50 \times 0.45 \times 0.18$ & $0.50 \times 0.41 \times 0.22$ & $0.41 \times 0.27 \times 0.08$ \\
\hline crystal system & triclinic & triclinic & monoclinic \\
\hline space group & $P-1$ & $P-1$ & $P 2_{1} / c$ \\
\hline$a[\AA]$ & $12.1096(4)$ & $13.2372(8)$ & $13.3883(5)$ \\
\hline$b[\AA]$ & $13.0805(4)$ & $13.2997(9)$ & $13.6790(4)$ \\
\hline$c[\AA]$ & $16.1502(5)$ & $14.8782(9)$ & $19.3600(8)$ \\
\hline$\alpha\left[^{\circ}\right]$ & $102.557(3)$ & $91.803(5)$ & 90 \\
\hline$\beta\left[^{\circ}\right]$ & $101.893(3)$ & $112.666(6)$ & $90.268(3)$ \\
\hline$\gamma\left[^{\circ}\right]$ & $105.462(3)$ & $105.470(5)$ & 90 \\
\hline$V\left[\AA^{3}\right]$ & $2309.88(13)$ & $2271.8(2)$ & $3545.5(2)$ \\
\hline$Z$ & 1 & 1 & 4 \\
\hline$\rho_{\text {calcd. }}\left[\mathrm{g} \mathrm{cm}^{-3}\right]$ & 1.583 & 1.737 & 1.584 \\
\hline$F(000)$ & 1126 & 1198 & 1748 \\
\hline$\mu\left[\mathrm{mm}^{-1}\right]$ & 1.337 & 2.297 & 1.122 \\
\hline$T_{\max } / T_{\min }$ & $0.8090 / 0.4728$ & $0.6293 / 0.2962$ & $0.0871 / 0.6235$ \\
\hline$h k l$ range & $\pm 15, \pm 16, \pm 20$ & $\pm 16, \pm 16,-17,+16$ & $\pm 16,-15,+17, \pm 24$ \\
\hline$\theta$ range $\left[{ }^{\circ}\right]$ & $1.35-26.72$ & $1.75-25.71^{\circ}$ & $1.52-26.78$ \\
\hline measured refl. & 27334 & 24303 & 39496 \\
\hline unique refl. $\left[R_{\text {int }}\right]$ & $9786[0.0590]$ & 8589 [0.0465] & $7516[0.0454]$ \\
\hline ref. param. / re straints & $593 / 15$ & $568 / 3$ & $479 / 0$ \\
\hline goodness-of-fit & 1.063 & 1.038 & 1.033 \\
\hline$R 1, w R 2(I>2 \sigma(I))$ & $0.0462,0.1251$ & $0.0550,0.1479$ & $0.0289,0.0683$ \\
\hline$R 1, w R 2$ (all data) & $0.0554,0.1295$ & $0.0700,0.1560$ & $0.0393,0.0711$ \\
\hline resid. el. dens. $\left[\mathrm{e} \AA^{-3}\right]$ & $1.684 /-0.914$ & $0.937 /-2.030$ & $0.584 /-0.354$ \\
\hline
\end{tabular}


Table 10.7. Crystal data and refinement details

\begin{tabular}{|c|c|c|}
\hline compound & 17 & 18 \\
\hline formula & $\mathrm{C}_{52} \mathrm{H}_{60} \mathrm{Cl}_{4} \mathrm{Mn}_{6} \mathrm{~N}_{12} \mathrm{O}_{14}$ & $\mathrm{C}_{43} \mathrm{H}_{35} \mathrm{Mn}_{2} \mathrm{Gd}_{2} \mathrm{~N}_{8} \mathrm{O}_{23}^{-}, \mathrm{C}_{6} \mathrm{H}_{16} \mathrm{~N}^{+}$ \\
\hline $\mathrm{M}_{\mathrm{r}}$ & 1548.56 & 1558.37 \\
\hline crystal size [mm] & $0.26 \times 0.13 \times 0.04$ & $0.50 \times 0.32 \times 0.30$ \\
\hline crystal system & monoclinic & monoclinic \\
\hline space group & $P 2_{1} / n$ & $P 2_{1} / c$ \\
\hline$a[\AA]$ & $12.5459(9)$ & $15.8277(5)$ \\
\hline$b[\AA]$ & $11.5237(5)$ & 17.4185() \\
\hline$c[\AA]$ & $21.5862(15)$ & $25.0884(11)$ \\
\hline$\alpha\left[^{\circ}\right]$ & 90 & 90 \\
\hline$\beta\left[^{\circ}\right]$ & $90.700(6)$ & $105.971(3)$ \\
\hline$\gamma\left[{ }^{\circ}\right]$ & 90 & 90 \\
\hline$V\left[\AA^{3}\right]$ & $3120.6(3)$ & $6649.8(4)$ \\
\hline$Z$ & 2 & 4 \\
\hline$\rho_{\text {calcd. }}\left[\mathrm{g} \mathrm{cm}^{-3}\right]$ & 1.648 & 1.557 \\
\hline$F(000)$ & 1572 & 3080 \\
\hline$\mu\left[\mathrm{mm}^{-1}\right]$ & 1.420 & 2.416 \\
\hline$T_{\max } / T_{\min }$ & $0.9268 / 0.7492$ & $0.5510 / 0.3838$ \\
\hline$h k l$ range & $\pm 15,-14,+13, \pm 27$ & $-18,+19, \pm 21, \pm 30$ \\
\hline$\theta$ range $\left[{ }^{\circ}\right]$ & $1.87-26.79$ & $1.34-25.63$ \\
\hline measu1red refl. & 39585 & 74071 \\
\hline unique refl. $\left[R_{\text {int }}\right]$ & $6621[0.0960]$ & $12549[0.0613]$ \\
\hline ref. param. / restraints & $430 / 4$ & $736 / 31$ \\
\hline goodness-of-fit & 1.082 & 1.058 \\
\hline$R 1, w R 2(I>2 \sigma(I))$ & $0.0570,0.1149$ & $0.0384,0.0937$ \\
\hline$R 1, w R 2$ (all data) & $0.0818,0.1241$ & $0.0456,0.0969$ \\
\hline resid. el. dens. $\left[\mathrm{e} \AA^{-3}\right]$ & $0.846 /-0.608$ & $0.755 /-1.313$ \\
\hline
\end{tabular}


Table 10.8. Crystal data and refinement details

\begin{tabular}{|c|c|c|}
\hline compound & 20 & 21 \\
\hline formula & $\begin{array}{l}\mathrm{C}_{43} \mathrm{H}_{35} \mathrm{Mn}_{2} \mathrm{Dy}_{2} \mathrm{~N}_{8} \mathrm{O}_{23}{ }^{-} \\
\mathrm{C}_{6} \mathrm{H}_{16} \mathrm{~N}^{+}\end{array}$ & $\mathrm{C}_{38.5} \mathrm{H}_{61} \mathrm{Cu}_{2} \mathrm{~N}_{4} \mathrm{O}_{6.5}$ \\
\hline $\mathrm{M}_{\mathrm{r}}$ & 1568.87 & 809.98 \\
\hline crystal size $[\mathrm{mm}]$ & $0.13 \times 0.08 \times 0.08$ & $0.48 \times 0.06 \times 0.06$ \\
\hline crystal system & monoclinic & monoclinic \\
\hline space group & $P 2_{1} / c$ & Pbcn \\
\hline$a[\AA]$ & $15.7037(8)$ & $31.3573(12)$ \\
\hline$b[\AA]$ & $17.4425(8)$ & $13.9485(7)$ \\
\hline$c[\AA]$ & $25.2451(16)$ & $9.4210(4)$ \\
\hline$\alpha\left[^{\circ}\right]$ & 90 & 90 \\
\hline$\beta\left[^{\circ}\right]$ & $106.406(5)$ & 90 \\
\hline$\gamma\left[^{\circ}\right]$ & 90 & 90 \\
\hline$V\left[\AA^{3}\right]$ & $6633.4(6)$ & $4120.6(3)$ \\
\hline$Z$ & 4 & 4 \\
\hline$\rho_{\text {calcd. }}\left[\mathrm{g} \mathrm{cm}^{-3}\right]$ & 1.571 & 1.306 \\
\hline$F(000)$ & 3096 & 1716 \\
\hline$\mu\left[\mathrm{mm}^{-1}\right]$ & 2.675 & 1.080 \\
\hline$T_{\max } / T_{\min }$ & $0.8419 / 0.5388$ & 0.9617 / 0.7845 \\
\hline$h k l$ range & $-19,+18,-18,+21, \pm 30$ & $\pm 38, \pm 16,+11,-10$ \\
\hline$\theta$ range $\left[{ }^{\circ}\right]$ & $1.35-25.69$ & $1.30-25.62$ \\
\hline measured refl. & 52199 & 20020 \\
\hline unique refl. [ $\left.R_{\text {int }}\right]$ & $12523[0.1037]$ & $3657[0.0739]$ \\
\hline ref. param. / restraints & $707 / 7$ & $245 / 5$ \\
\hline goodness-of-fit & 1.013 & 1.032 \\
\hline$R 1, w R 2(I>2 \sigma(I))$ & $0.0507,0.0919$ & $0.0596,0.1363$ \\
\hline$R 1, w R 2$ (all data) & $0.0843,0.0993$ & $0.0920,0.1507$ \\
\hline resid. el. dens. $\left[\mathrm{e} \AA^{-3}\right]$ & $1.090 /-1.527$ & $0.686 /-0.583$ \\
\hline
\end{tabular}


Table 10.9. Crystal data and refinement details

\begin{tabular}{|c|c|c|}
\hline compound & 22 & 23 \\
\hline formula & $\mathrm{C}_{38.33} \mathrm{H}_{51.33} \mathrm{Cu}_{2} \mathrm{~N}_{4} \mathrm{O}_{4} \mathrm{Cl}_{4}$ & $\mathrm{C}_{38.33} \mathrm{H}_{51.33} \mathrm{Ni}_{2} \mathrm{~N}_{4} \mathrm{O}_{4} \mathrm{Cl}_{4}$ \\
\hline $\mathrm{M}_{\mathrm{r}}$ & 901.05 & 891.39 \\
\hline crystal size [mm] & $0.50 \times 0.45 \times 0.34$ & $0.40 \times 0.16 \times 0.10$ \\
\hline crystal system & triclinic & triclinic \\
\hline space group & $P-1$ & $P-1$ \\
\hline$a[\AA]$ & $15.1659(4)$ & $15.1453(5)$ \\
\hline$b[\AA]$ & $18.8380(5)$ & $18.8054(7)$ \\
\hline$c[\AA]$ & $25.8852(6)$ & $25.7598(8)$ \\
\hline$\alpha\left[^{\circ}\right]$ & $71.849(2)$ & $71.991(3)$ \\
\hline$\beta\left[^{\circ}\right]$ & $73.848(2)$ & $74.363(3)$ \\
\hline$\gamma\left[^{\circ}\right]$ & $68.838(2)$ & $68.159(3)$ \\
\hline$V\left[\AA^{3}\right]$ & $6437.8(3)$ & $6376.5(4)$ \\
\hline$Z$ & 6 & 6 \\
\hline$\rho_{\text {calcd. }}\left[\mathrm{g} \mathrm{cm}^{-3}\right]$ & 1.394 & 1.393 \\
\hline$F(000)$ & 2804 & 2792 \\
\hline$\mu\left[\mathrm{mm}^{-1}\right]$ & 1.282 & 1.179 \\
\hline$T_{\max } / T_{\min }$ & $0.7197 / 0.4592$ & $0.8843 / 0.7387$ \\
\hline$h k l$ range & $\pm 19, \pm 23,+29,-32$ & $-19,+15, \pm 23,-31,+32$ \\
\hline$\theta$ range $\left[{ }^{\circ}\right]$ & $1.47-26.79$ & $1.30-26.75$ \\
\hline measured refl. & 73144 & 53181 \\
\hline unique refl. $\left[R_{\text {int }}\right]$ & $27153[0.0370]$ & $26515[0.0567]$ \\
\hline ref. param. / restraints & $1442 / 23$ & $1441 / 25$ \\
\hline goodness-of-fit & 1.037 & 1.023 \\
\hline$R 1, w R 2(I>2 \sigma(I))$ & $0.0391,0.1019$ & $0.0621,0.1047$ \\
\hline$R 1, w R 2$ (all data) & $0.0469,0.1054$ & $0.1179,0.1160$ \\
\hline resid. el. dens. [e $\left.\AA^{-3}\right]$ & $1.233 /-1.092$ & $1.999 /-1.043$ \\
\hline
\end{tabular}




\section{Bibliography}

${ }^{1}$ a) B. G. Morin, C. Hahm, A. J. Epstein, J. S. Miller, J. Appl. Phys. 1994, 75, 5782; b) J. S. Miller. Inorg. Chem. 2000, 39 , 4392.

2 a) J. Larionova, O. Kahn, S. Golhen, L. Ouahab, R. Clérac, J. Am. Chem. Soc. 1999, 121, 3349; b) M. Ohba, N. Usuki, N. Fukita, H. Okawa, Angew.Chem. Int. Ed. 1999, 38, 1795; c) J. S. Miller, Adv. Mater. 2002, 14, 1105; d) J. R. Galán-Mascarós, K. R. Dunbar, Angew. Chem. Int. Ed. 2003, 42, 2289; e) H. Miyasaka, H. Ieda, N. Matsumoto, K. Sugiura, M. Yamashita, Inorg. Chem. 2003, 42, 3509.

${ }^{3}$ a) J. K. Kim; J. M. Lim, Y.-K. Choi, Y. Do, Angew. Chem. Int.Ed. Engl. 1996, 35, 998; b) F. A. Mantner, R. Cortes, L. Lezama, T. Rojo, Angew. Chem. Int. Ed. Engl. 1996, 35, 78; c) H. Miyasaka, N. Matsumoto, H. Okawa, N. Re, E. Gallo, C. Floriani, J. Am.Chem. Soc. 1996, 118, 981; (d) W. R. Entley, G. S. Girolami, Science 1995, 268, 397.

${ }^{4}$ a) G. S. Papaefstathiou, R. Vicente, C. P. Raptopoulou, A. Terzis, A. Escuer, S. P. Perlepes, Eur. J. Inorg. Chem, 2002, 2488; b) Y. Kim, D. P. Jung, Inorg. Chem. 2000, 39, 1470; c) E. Lee, Y. Kim, D. Y. Jung, Inorg. Chem. 2002, 41, 501; d) T. F Liu, H. L. Sun, S. Gao, S.W. Zhang, T. C. Lau, Inorg. Chem. 2003, 42, 4792; d) M.-L. Tong, S. Kitagawa, H.-C. Chang, M. Obha, Chem. Comm. 2004, 418; e) W.-W. Ni, Z.- H. Ni, A.-L. Cui, X. Liang, H.-Z. Kou, Inorg. Chem. 2007, 46, 22.

5 a) T. Lis, Acta Crystallogr. Sect. B 1980, 36, 2042; b) R. Sessoli, H.L. Tsai, A.R. Schake, S. Wang, J.B. Vincent, K. Folting, D. Gatteschi, G. Christou, D.N. Hendrickson, J. Am. Chem. Soc. 1993, 115, 1804; c) R. Sessoli, D. Gatteschi, M.A. Novak, A. Caneschi, Nature 1993, 356, 141; d) L. Thomas, L. Lionti, R. Ballou, D. Gatteschi, R. Sessoli, B. Barbara, Nature 1996, 383, 145 .

${ }^{6}$ J. S. Miller, A. J. Epstein, Angew. Chem., Int. Ed. 1994, 33, 385.

7 a) J. C. Longuet-Higgins, J. Chem. Phys. 1950, 18, 265; b) H. Iwamura, Adv. Phys. Org. Chem. 1990, 26, 179; c) A. Rajca, Chem. Rev. 1994, 94, 871; d) S. Rajca, A. Rajca, J. Am. Chem. Soc. 1995, 117, 9172; e) A. Rajca, S. Rajca, J. Am. Chem. Soc. 1996, 118, 8121.

${ }^{8}$ Selected examples: a) V. A. Ung, A. M. W. Cargill Thompson, D. A. Bardwell, D. Gatteschi, J. C. Jeffery, J. A. McCleverty, F. Totti, M. D. Ward, Inorg. Chem. 1997, 36, 3447; 
b) T. Ishida, S. Mitsubori, T. Nogami, N. Takeda, M. Ishikawa, H. Iwamura, Inorg. Chem. 2001, 40, 7059; c) X. Ottenwaelder, J. Cano, Y. Journax, E. Riviére, C. Brennan, M. Nielrich, R. Ruiz-García, Angew. Chem. Int. Ed. 2004, 43, 850; d) M. Pascu, F. Lloret, N. Avarvari, M. Julve, M. Andruh, Inorg. Chem. 2004, 43, 5189; e) T. Glaser, M. Heidemeier, S. Grimme, E. Bill, Inorg. Chem. 2004, 43, 5192; f) F. Lloret, G. De Munno, J. Cano, R. Ruiz, A. Caneshi, Angew. Chem. Int. Ed. 1998, 37, 135; g) E. Pardo, K. Bernot, M. Julve, F. Lloret, J. Cano, R. Ruiz-García, F. S. Delgado, C. Ruiz-Perez, X. Ottenwaelder, Y. Journaux, Angew. Chem. Int. Ed. 2004, 43, 2768; h) E. Pardo, J. Faus, M. Julve, F. Lloret, M. C. Munoz, J. Cano, X. Ottenwaelder, Y. Journaux, R. Carrasco, G. Blay, I. Fernandez, R. Ruiz-Garcia, J. Am. Chem. Soc. 2003, 125, 10770; i) A. R. Paital, T. Mitra, D. Ray, W. T. Wong, J. Ribas-Arino, J. J. Nova, J. Ribas, G. Aromí, Chem. Commun. 2005, 5172; j) I. Fernandez, R. Ruiz, J. Faus, M. Julve, F. Lloret, J. Cano, X. Ottenwaelder, Y. Journaux, M. Carmen Munoz, Angew. Chem. Int. Ed. 2001, 40, 3039.

${ }^{9}$ a) E. F. Hasty, L. J. Wilson, D. N. Hendrickson, Inorg. Chem. 1978, 17, 1834; b) T. Ishida, T. Kawakami, S. Mitsubori, T. Nogami, K. Yamaguchi, H. Iwamura, J. Chem. Soc. Dalton Trans. 2002, 3177; c) M. Matsushita, T. Yasuda, R. Kawano, T. Kawai, T. Iyoda, Chem. Lett. 2000, 812; d) H. Torayama, T. Nishida, H. Asada, M. Fujiwara, T. Matushita, Polyhedron, 1997, 16, 3787; e) A. Dei, D. Gatteschi, C. Sangregorio, L. Sorace, M. G. F. Vaz, Inorg. Chem. 2003, 42, 1701; f) A. Mederos, S. Dominguez, R. Hernádez-Molina, J. Sanchiz, F. Brito, Coord. Chem. Rev. 1999, 193-195, 857 and references therein.

${ }^{10}$ S. Mitsubori, T. Ishida, T. Nogami, H. Iwamura, Chem. Lett. 1994, 94, 285-288.

11 a) T. Glaser, M. Gerenkamp, R. Fröhlich, Angew.Chem. Int. Ed. 2002, 41, 3823; b) T. Glaser, M. Heidemeier, T. Lügger, Dalton Trans. 2003, 2381; (c) T. Glaser, M. Heidemeier, J. B. H. Strautmann, H. Bögge, A. Stammler, E. Krickemeyer, R. Huenerbein, S. Grimme, E. Bothe, E. Bill, Chem. Eur. J. 2007, 13, 9191.

12 (a) B. F. Fieselmann, D. N. Hendrickson, G. D. Stucky, Inorg. Chem. 1978, 17, 1841; (b) D. R. Corbin, L. B. Francesconi, D. H. Hendrickson, G. D. Stucky, Inorg. Chem. 1979, 18, 3069; (c) L. B. Francesconi, D. R. Corbin, D. H. Hendrickson, G. D. Stucky, Inorg. Chem. 1979, 18, 3074; (d) G. Glatz, Diplomarbeit, Göttingen, 2005. 
13 a) R. B. Stuart, R. Robson, Angew. Chem. Int. Ed. 1998, 37, 1460; b) M. Bertelli, L. Carlucci, G. Ciani, D. M. Proserpio, M. Davide and A. Sironi, J. Mater. Chem. 1997, 7, 1271; c) B. F. Abrahams, S. R. Batten, M. J. Grannas, H. Hamit, B. F. Hoskins, R. Robson, Angew. Chem. Int. Ed. 1999, 38, 1475.

14 a) A. M. Garcia, D. M. Bassani, J.-M. Lehn, G. Baum, D. Fenske, Chem. Eur. J. 1999, 5, 1234; b) S. Furukawa, M. Ohba, S. Kitagawa, Chem. Commun. 2005, 865; c) E. Bosch, C. L. Barnes, Inorg. Chem. 2002, 41, 2543; d) R. D. Pike, B. D. Borne, J. T. Maeyer, A. L. Rheingold, Inorg. Chem. 2002, 41, 631; e) A. J. Blake, N. R. Brooks, N. R. Champness, P.A. Cooke, A. M. Deveson, D. Fenske, P. Hubberstey, W.-S. Li, M. Schröder, J. Chem. Soc., Dalton Trans. 1999, 2103; f) T. Glaser, T. Lügger, R. Fröhlich, Eur. J. Inorg. Chem. 2004, 394; g) M. Trivedi, D. S. Pandey, R.-Q. Zou, Q. Xu, Inorg. Chem. Commun. 2008, 11, 526; h) Y.-F. Han, Y.-B. Huang, Y.-J. Lin, G.-X. Jin, Organometallics 2008, 27, 961; i) S. Kar, B. Pradhan, R. K. Sinha, T. Kundu, P. Kodgire, K. K. Rao, V. G. Puranik, G. K. Lahiri, Dalton Trans. 2004, 1752; j) S. Aoki, M. Zulkefeli, M. Shiro, E. Kimura, Proc. Natl. Acad. Sci. 2002, 99, 4894; k) C.-K. Chan, K.-K. Cheung, C.-M. Che, Chem. Commun. 1996, 227; 1) Z. Trávníček, J. Marek, S. A Čermáková, Acta Cryst. 2007, E63, m1742.

15 a) J. Klingele, S. Dechert, F. Meyer, Coord. Chem. Rev. 2009, 253, 2698-2741.

${ }^{16}$ R. Mukherjee, Coord. Chem. Rev. 2000, 203, 151.

${ }^{17}$ a) M. Viciano-Chumillas, S. Tanase, G. Aromí, J. M. M. Smits, R. de Gelder, X. Solans, E. Bouwman, J. Reedijk, Eur. J. Inorg. Chem. 2007, 2635; b) M. Viciano-Chumillas, S. Tanase, I. Mutikainen, M. Turpeinen, L. J. de Jongh, J. Reedijk, Inorg. Chem. 2008, 47, 5919; c) S. Tanase, G. Aromí, E. Bouwman, H. Kooijman, A. L. Spek, J. Reedijk, Chem. Commun. 2005, 3147; d) Y.-L. Bai, J. Tao, W. Wernsdorfer, O. Sato, R.-B. Huang, L.-S. Zheng, J. Am. Chem. Soc. 2006, 128, 16428; e) C.-M. Liu, D.-Q. Zhang, D.-B. Zhu, Chem. Commun. 2008, 368.

18 a) R. L. Carlin, Magnetochemistry, Springer-Verlag, 1986; b) O. Kahn, Molecular Manetism, VCH Publ. 1993; c) C. Kittel, Introduction to solid state physics, 2nd Ed. J. Wiley and Sons, New York, 1953.

${ }^{19}$ J.-M. Lehn, Supramolecular Chemistry: Concepts and Perspectives, VCH, publ, 1995.

20 a) R. Winpenny (ed), Str. Bonding 2006, 122; b) D. Gatteschi, R. Sessoli, J. Villain, Molecular Nanomagnets, Oxford University Press, 2006; c) Magnetism: Molecules to 
Materials. Molecule Based Materials, Vol. 2 (Eds.: J. S. Miller, M. Drillon), Wiley-VCH, Weinheim, 2001.

${ }^{21}$ a) G. Christou, D. Gatteschi, and D. N. Hendrickson, MRS Bulletin, 2000, 25, 66; b) J. R. Long, In Chemistry of Nanostructured Materials; World Scientific: Hong Kong, 2003, pp 291-315 and references therein.

${ }^{22}$ R. Bagai, G. Christou, Chem. Soc. Rev. 2009, 38, 1011.

${ }^{23}$ Gatteschi, D.; Sessoli, R. Angew. Chem. Int. Ed. 2003, 42, 268.

${ }^{24}$ a) V. Marvaud, C. Decroix, A. Scuiller, F. Tuyeras, C. Guyardduhayon, J. Vaissermann, M. Marrot, F. Gonnet, M. Verdaguer, Chem. Eur. J. 2003, 9, 1692; b) T. Mallah, C. Auberger, M. Verdaguer, P. Veillet, J. Chem. Soc. Chem. Commun. 1995, 61.

${ }^{25}$ a) E. Breuning, M. Ruben, J.-M. Lehn, F. Renz, Y. Garcia, V. Ksenofontov, P. Gutlich, E. Wegelius, K. Rissanen, Angew. Chem. Int. Ed. 2000, 39, 2504; b) L. Zhao, Z. Q. Xu, H. Grove, V. A. Milway, L. N. Dawe, T. S. M. Abedin, L. K. Thompson, T. L. Kelly, R. G. Harvey, D. O. Miller, L. Weeks, J. G. Shapter, K. J. Pope, Inorg. Chem. 2004, 43, 3812; c) T. Glaser, M. Heidemeier, T. Weyhermüller, R.-D. Hoffmann, H. Rupp, P. Müller, Angew. Chem. Int. Ed. 2006, 45, 6033.

${ }^{26}$ (a) A. J. Tasiopoulos, A. Vinslava, W. Wernsdorfer, K. A. Abboud, G. Christou, Angew. Chem. Int. Ed. 2004, 43, 2117; (b) A. M. Ako, I. J. Hewitt, V. Mereacre, R. Clérac, W. Wernsdorfer, C. E. Anson, A. K. Powell, Angew.Chem. Int. Ed. 2006, 45, 4926; (c) T. Glaser, I. Liratzis, A. M. Ako, A. K. Powell, Coord. Chem. Rev. 2009, 253, 2296; (d) G. E. Kostakis, A. K. Powell, Coord. Chem. Rev. 2009, 253, 2686; (e) A. M. Ako, V. Mereacre, R. Clérac, W. Wernsdorfer, I. J. Hewitt, C. E. Anson, A. K. Powell, Chem. Commun. 2009, 544; (f) A. M. Ako, Valeriu Mereacre, R. Clérac, I. J. Hewitt, Y. Lan, G. Buth, C. E. Anson, A. K. Powel, Inorg. Chem. 2009, 48, 6713.

${ }^{27}$ R. Sessoli, A. K. Powell, Coord. Chem. Rev. 2009, 253, 2328.

28 a) P. Gamez, J. Reedijk, Eur. J. Inorg. Chem. 2006, 29; b) T. J. Mooibroek, P. Gamez, Inorg. Chim. Acta 2007, 360, 381.

${ }^{29}$ A. Ranganathan, B. C. Heisen, I. Dix. F. Meyer, Chem. Commun. 2007, 3637. 
${ }^{30}$ a) S. Demeshko, S. Dechert, F. Meyer, J. Am. Chem. Soc., 2004, 126, 4508; b) T. J. Mooibroek, C. A. Black, P. Gamez, J. Reedijk, Cryst. Growth Des. 2008, 8, 1082; c) B. L. Schottel, H. T. Chifotides, K. R. Dunbar, Chem. Soc. Rev. 2008, 37, 68.

${ }^{31}$ J. A. Zerkowski, C. T. Seto, G. M. Whitesides, J. Am. Chem. Soc. 1992, 114, 5473.

32 a) L. Cronin, Angew. Chem. Int. Ed. 2006, 45, 3576; b) M. Yoshizawa, M. Fujita, Pure Appl. Chem. 2005, 77, 1107.

33 a) I. E. Lerner, J. S. Lippard, Inorg. Chem., 1977, 16, 1546; b) D. C. de Castro Gomes, H. O. Stumpf, F. Lloret, M. Julve, V. Gonzales, H. Adams, J. A. Thomas, Inorg. Chim. Acta 2005, 358, 1113; c) X.-P. Zhou, D. Li, S.-L. Zheng, X. Zhang, T. Wu, Inorg. Chem. 2006, 45, 7119.

${ }^{34}$ R. Fink, Y. Heischkel, M. Thelakkat, H.-W. Schmidt, C. Jonda, M. Hüppauff, Chem. Mater. 1998, 10,3620 .

${ }^{35}$ P. Chaudhuri, K. Wieghardt, Prog. Inorg. Chem. 2001, 50, 151.

${ }^{36}$ a) R. C. Pratt, T. D. P. Stack, J. Am. Chem. Soc. 2003, 125, 8716; b) R. C. Pratt, T. D. P. Stack, Inorg. Chem. 2005, 44, 2367; c) T. Storr, P. Verma, C. R. Pratt, C. E. Wasinger, Y. Shimazaki, T. D. P. Stack, J. Am. Chem. Soc. 2008, 130, 15448; d) L. M. Mirica, M. Vance, D. J. Rudd, B. Hedman, K. O. Hodgson, E. I. Solomon, T. D. P. Stack, Science 2005, 308, 1890; e) B. T. Op't Holt, M. A. Vance, L. M. Mirica, D. E. Heppner, T. D. P. Stack, E. I. Solomon, J. Am. Chem. Soc. 2009, 131, 6421.

${ }^{37}$ X. Zhao, X.-Z. Wang, X.-K. Jiang, Y.-Q. Chen, Z.-T. Li, G.-J. Chen, J. Am. Chem. Soc. 2003, 125,15128 .

${ }^{38}$ M. Diaz, A. Cobas, E. Guitian, L. Castedo, Eu. J. Org. Chem. 2001, 4543.

${ }^{39}$ a) G. R. Desiraju, T. Steiner, The Weak Hydrogen Bond; Oxford University Press: Oxford, 1999 and refs therein; b) G. R. Desiraju, Acc. Chem. Res. 2002, 35, 565 and refs therein.

${ }^{40}$ J. Ramirezirez, A.-M. Stadler, N. Kyritskas, J.-M. Lehn, Chem. Commun. 2007, 237.

${ }^{41}$ a) M. Rudolph, S. Feldberg, DigiSim3, Version 3.03, DigiSim3, Version 3.03, Bioanalytical Systems, Inc.: 1994: 
${ }^{42}$ A. S. Baranski, W. R. Fawcett, C. M. Gilbert, Anal. Chem. 1985, 57, 166.

${ }^{43}$ a) T. Kurahashi, A. Kikuchi, T. Tosha, Y. Shiro, T. Kitagawa, H. Fujii, Inorg. Chem. 2008, $47,1674$.

${ }^{44}$ a) J.A. Halfen, B. A. Jazdzewski, S. Mahapatra, L. M. Berreau, E. C. Wilkinson, L. Que, Jr., W. B. Tolman, J. Am. Chem. Soc. 1997, 35, 8217; b) J. Hockertz, S. Steenken, K. Wieghardt, P. Hildebrandt, J. Am. Chem. Soc. 1993, 115, 11222; c) A. Sokolowski, H. Leutbecher, T. Weyhermüller, R. Schnepf, E. Bothe, E. Bill, P. Hildebrandt, K. Wieghardt, J. Biol. Inorg. Chem. 1997, 2, 444.

${ }^{45}$ T. Ishida, T. Kawakami, S.-I. Mitsubori, T. Nogami, K. Yamaguchi, H. Iwamura, J. Chem. Soc. Dalton Trans. 2002, 3177.

${ }^{46}$ K. S. Murray, Coord. Chem. Rev. 1974, 12, 1-35.

${ }^{47}$ A. X. Trautwein, E. Bill, E. L. Bominaar, H. Winkler, Structure and Bonding 1991, 78, 1.

${ }^{48}$ A. Escuer, G. Aromí, Eur. J. Inorg. Chem. 2006, 4721.

${ }^{49}$ R. D. Willet, D. Gatteschi, O. Kahn (Eds.), Magneto-Structural Correlations in Exchange Coupled Systems, NATO ASI Series, Reidel, Dordrecht 1985.

50 a) M. M. Turnbull, T. Sugimoto, L. K. Thompson (Eds.), Molecular-Based Magnetic Materials: Theory, Techniques and Applications, ACS Symposium Series, 644, ACS, Washington 1996; b) A. Caneschi, D. Gatteschi, L. Pardi, R. Sessoli, Clusters, Chains and Layered Molecules: the Chemist's Way to Magnetic Materials, in: A. F. Williams (Ed.), Perspectives in Coordination Chemistry, VCH, Weinheim 1992.

${ }^{51}$ J. Ribas, A. Escuer, M. Monfort, R. Vicente, R. Cortes, L. Lezama, T. Rojo, Coord. Chem. Rev. 1999, 193-195, 1027.

52 a) Z.-H. Ni, H.-Z. Kou, L. Zheng, Y.-H. Zhao, L.-F. Zhang, R.-J. Wang, A.-L. Cui, O. Sato, Inorg. Chem. 2005, 44, 4728; b) A. Escuer, R. Vicente, J. Ribas, X. Solans, Inorg. Chem. 1995, 34, 1793; c) M. A. Halcrow, J. C. Huffman, G. Christou, Angew. Chem. Int. Ed. Engl. 1995, 34, 889; d) F. Meyer, S. Demeshko, G. Leibeling, B. Kersting, E. Kaifer, Chem. Eur. J. 2005, 11, 1518; e) G. S. Papaefstathiou, S. P. Perlepes, A. Escuer, R. Vicente, M. FontBardia, X. Solans, Angew. Chem. Int. Ed. 2001, 40, 884; f) F. Meyer, P. Kircher, H. Pritzkow, Chem. Commun. 2003, 774; g) S. Demeshko, G. Leibeling, W. Maringgele, F. Meyer, C. 
Mennerich, H.-H. Klauss, H. Pritzkow, Inorg. Chem. 2005, 44, 519; h) G. Leibeling, S. Demeshko, S.Dechert, F. Meyer, Angew. Chem. Int. Ed. 2005, 44, 7273.

${ }^{53}$ M. A. S. Goher, J. Cano, Y. Journaux, M. A. M. Abu-Youssef, F. A. Mautner, A. Escuer, R. Vicente, Chem. Eur. J. 2000, 6, 778 and references therein.

54 a) T. K. Maji, W. Kaneko, M. Ohba, S. Kitagawa, Chem. Commun. 2005, 4613; b) A. Das, G. M. Rosair, M. S. E. Fallah, J. Ribas, S. Mitra, Inorg. Chem. 2006, 45, 3301.

55 a) J. Ribas, M. Monfort, X. Solans, Inorg. Chem. 1993, 32, 3557; b) Z.-H. Zhang, X.-H. Bu, Z.-H. Ma, W.-M. Bu, Y. Tang, Q.-H. Zhao, Polyhedron. 2000, 19, 1959.

${ }^{56}$ a) O. Kahn, Struct. Bonding 1987, 68, 89; b) B. J. Goodenough, Phys. Rev. 1955, 100, 564; c) J. B. Goodenouh, J. Phys. Chem. Solids 1958, 6, 287; d) J. Kanamori, J. Phys. Chem. Solids 1959, 10, 87; e) P. A. Ginsberg, Inorg. Chim. Acta Rev. 1971, 5, 45.

57 a) P. Chaudhuri, Coord. Chem. Rev. 2003, 243, 143; b) H. Okawa, H. Furutachi, D. E. Fenton, Coord. Chem. Rev. 1998, 174, 51; c) P. A. Figato, S. Tamburini, Coord. Chem. Rev. 2004, 248, 1717.

58 a) T. Shiga, H. Oshio, Sci. Technol. Adv. Mater. 2005, 6, 565, and reference therein; b) K. Mitsumoto, S. Koizumi, T. Shiga, H. Nishikawa, Y. Chi, H. Oshio, Chem. Lett. 2007, 1154; c) Y. M. Chumakov, V. N. Biyushkin, T. I. Malinovskii, S. Kulemu, V. I. Tsapkov, M. S. Popov, N. M. Samus, Koord. Khim. 1990, 16, 945.

${ }^{59}$ A.-K. Pleier, H. Glas, M. Grosche, P. Sirsch, W. R. Thiel, Synthesis 2001, 1, 55.

${ }^{60}$ B. D. M. Cunningham, P. R. Lowe, M. D. Threadgill, J. Chem. Soc. Perkin Trans. 2, 1989, 1275 .

${ }^{61}$ R. Kowalczyk, J. Skarzewski, Pol. J. Chem. 2007, 81, 1987.

${ }^{62}$ T. K. Ronson, H. Adams, M. D. Ward, Inorg. Chim. Acta 2005, 358, 1943.

${ }^{63}$ a) K. S. Murray, Adv. Inorg. Chem. 1995, 43, 261; b) M. A. Halcrow, J. S. Sun, J. C. Huffman, G. Christou, Inorg. Chem. 1995, 34, 4167; c) M. S. El Fallah, E. Rentschler, A. Caneschi, D. Gatteschi, Inorg. Chim. Acta 1996, 247, 231; d) S. Mukherjee, T. Weyhermüller, 
E. Bothe, K. Wieghardt, P. Chaudhuri, Eur. J. Inorg. Chem. 2003, 863; e) J. M. ClementeJuan, B. Chansou, B. Donnadieu, J. P. Tuchagues, Inorg. Chem. 2000, 39, 5515.

64 a) H. Oshio, N. Hoshino, T. Ito, M. Nakano, J. Am. Chem. Soc. 2006, 126, 8805; b) A. Sieber, C. Boskovic, R. Bircher, O. Waldmann, S. T. Ochsenbein, G. Chaboussant, H. U. Güdel, N. Kirchner, J. van Slageren, W. Wernsdorfer, A. Neels, H. Stoeckli-Evans, S. Janssen, F. Juranyi, H. Mutka, Inorg. Chem. 2005, 44, 4315.

${ }^{65}$ O. Waldmann, M. Ruben, U. Ziener, P. Müller, J.-M. Lehn, Inorg. Chem. 2006, 45, 6535.

${ }^{66}$ a) P. Lainé, A. Gourdon, J.-P. Launay, Inorg. Chem. 1995, 34, 5129; b) D. Dobrzyńska, M. Duczmal, L. B. Jerzykiewicz, J. Warchulska, K. Drabent, Eur. J. Inorg. Chem. 2004, 110.

${ }^{67}$ C. J. Milios, A. Vinslava, W. Wernsdorfer, S. Moggach, S. Parsons, S. P. Perlepes, G. Christou, E. K. Brechin, J. Am. Chem. Soc. 2007, 129, 2754.

${ }^{68}$ a) E. Cremades, J. Cano, E. Ruiz, G. Rajaraman, C. J. Milios, E. K. Brechin, Inorg. Chem. 2009, 48, 8012; b) R. Inglis, L. F. Jones, C. J. Milios, S. Datta, A. Collins, S. Parsons, W. Wernsdorfer, S. Hill, S. P. Perlepes, S. Piligkos, E. K. Brechin, Dalton Trans. 2009, 3403.

${ }^{69}$ W. Wernsdorfer, N. Aliaga-Alcalde, D. N. Hendrickson, G. Christou, Nature 2002, 416, 406.

70 a) A. Yamaguchi, N. Kusumi, H. Ishimoto, H. Mitamura, T. Goto, N. Mori, M. Nakano, K. Awaga, J. Yoo, D. N. Hendrickson, G. Christou, J. Phys. Soc. Jpn. 2002, 71, 414 ; b) M. Affronte, J. C. Lasjaunias, W. Wernsdorfer, R. Sessoli, D. Gatteschi, S. L. Heath, A. Fort, A. Rettori, Phys. Rev. B 2002, 66, 064408; c) M. Dey, C. P. Rao, P. K. Saarenketo, K. Rissanen, Inorg. Chem. Commun. 2002, 5, 924.

71 C. Boskovic, R. Bircher, P. L. W. Tregenna-Piggott, H. U. Güdel, C. Paulsen, W. Wernsdorfer, A.-L. Barra, E. Khatsko, A. Neels, H. Stoeckli-Evans, J. Am. Chem. Soc. 2003, $125,14046$.

${ }^{72}$ H. Miyasaka, M. Yamashita, Dalton Trans. 2007, 399.

73 a) R. Clérac, H. Miyasaka, M. Yamashita, C. Coulon, J. Am. Chem. Soc.2002, 124, 12837; b) P. L. Feng, C. C. Beedle, W. Wernsdorfer, C. Koo, M. Nakano, S. Hill, D. N. Hendrickson, Inorg. Chem. 2007, 46, 8126; c) H. Oshio, M. Nihei, A. Yoshida, H. Nojiri, M. Nakano, A. 
Yamaguchi, Y. Karaki, H. Ishimoto, Chem. Eur. J. 2004, 11, 843; d) D. Li, S. Parkin, G. Wang, T. G. Yee, R. Clérac, W. Wernsdorfer, M. S. Holmes, J. Am. Chem. Soc. 2006, 128, 4214; e) H. Oshio, M. Nihei, S. Koizumi, T. Shiga, H. Nojiri, M. Nakano, N. Shirakawa, M. Akatsu, J. Am. Chem. Soc. 2005, 127, 4568.

${ }^{74}$ W. Liu, H. H. Thorp, Inorg. Chem. 1993, 32, 4102.

${ }^{75}$ Although hydrogen atoms could not be located in $\mathbf{1 4} \cdot 8 \mathrm{CH}_{2} \mathrm{Cl}_{2}$, an $\mathrm{H}$-bonding pattern similar to the situation in $\mathbf{1 4} \cdot 6 \mathrm{C}_{2} \mathrm{H}_{4} \mathrm{Cl}_{2}$ and $\mathbf{1 5} \cdot 8 \mathrm{CH}_{2} \mathrm{Cl}_{2}$ is likely.

${ }^{76}$ C. F. Macrae, I. J. Bruno, J. A. Chisholm, P. R. Edgington, P. McCabe, E. Pidcock, L. Rodriguez-Monge, R. Taylor, J. van de Streek, P. A. Wood, J. Appl. Cryst. 2008, 41, 466.

${ }^{77}$ A. K. Geim, S. V. Dubonos, J. G. S. Lok, I. V. Grigorieva, J. C. Maan, L. T. Hansen, P. E. Lindelof, Appl. Phys. Lett. 1997, 71, 2379.

${ }^{78}$ N. E. Chakov, W. Wernsdorfer, K. A. Abboud, G. Christou, Inorg. Chem. 2004, 43, 5919, and references therein.

${ }^{79}$ A. Abragam, B. Bleaney, Electron Paramagnetic Resonance of Transition Ions, Oxford University Press, London, 1970.

${ }^{80}$ A. Bencini, D. Gatteschi, EPR of Exchange Coupled Systems, Springer, 1990.

${ }^{81}$ P. Chaudhuri, V. Kataev, B. Büchner, H.-H. Klauss, B. Kersting, F. Meyer, Coord. Chem. Rev. 2009, 253, 2261.

${ }^{82}$ S. Stoll, A. Schweiger, J. Magn. Reson. 2006, 178(1), 42.

${ }^{83}$ a) M. Murugesu, R. Clérac, C. E. Anson, A. K. Powell, Inorg. Chem. 2004, 43, 7269; b) M. Murugesu, R. Clérac, C. E. Anson, A. K. Powell, Chem. Commun. 2004, 1598.

${ }^{84}$ a) M. Murugesu, M. Habrych, W. Wernsdorfer, K. A. Abboud, G. Christou, J. Am. Chem. Soc. 2004, 126, 4766; b) M. Soler, W. Wernsdorfer, K. Folting, M. Pink, G. Christou, J. Am. Chem. Soc. 2004, 126, 2156; c) A. J. Tasiopoulos, A. Vinslava, W. Wernsdorfer, K. A. Abboud, G. Christou, Angew. Chem. Int. Ed. 2004, 43, 2117; d) Y. Li, W. Wernsdorfer, R. Clérac, I. J. Hewitt, C. E. Anson, A. K. Powell, Inorg. Chem. 2006, 45, 2376.

${ }^{85}$ O. Waldmann, Inorg. Chem. 2007, 46, 10035. 
${ }^{86}$ T. C. Stamatatos, K. A. Abboud, W. Wernsdorfer, G. Christou, Angew. Chem. Int. Ed. 2006, 45, 4134.

${ }^{87}$ A. M. Ako, I. J. Hewitt, V. Mereacre, R. Clérac, W. Wernsdorfer, C. E. Anson, A. K. Powell, Angew. Chem. Int. Ed. 2006, 45, 4926.

88 a) A. Mishra, W. Wernsdorfer, K. A. Abboud, G. Christou, J. Am. Chem. Soc. 2004, 126, 15648; b) A. Mishra, W. Wernsdorfer, S. Parson, G. Christou, E. Brechin, Chem. Commun. 2005, 2086; c) T. C. Stamatatos, S. J. Teat, W. Wernsdorfer, G. Christou, Angew. Chem. Int. Ed. 2009, 48, 521.

${ }^{89}$ a) V. M. Mereacre, A. M. Ako, R. Clérac, W. Wernsdorfer, G. Filoti, J. Bartolome, C. E. Anson, A. K. Powell, J. Am. Chem. Soc. 2007, 129, 9248; b) V. Mereacre, A. M. Ako, R. Clérac, W. Wernsdorfer, I. J. Hewitt, C. E. Anson, A. K. Powell, Chem. Eur. J. 2008, 14, 3577.

${ }^{90}$ C. M. Zaleski, E. C. Depperman, J. W. Kampf, M. L. Kirk, V. L. Pecoraro, Angew. Chem. Int. Ed. 2004, 43, 3912.

${ }^{91}$ P.-H. Lin, T. J. Burchell, R. Clérac, M. Murugesu, Angew. Chem. Int. Ed. 2008, 47, 8848.

${ }^{92}$ X. S. Tan, J. Chen, Z. N. Chen, P. J. Zheng, W. X. Tang, Inorg. Chim. Acta 1995, 234, 27.

93 a) J. Stubbe, W. A. van der Donk, Chem. Rev. 1998, 98, 705; b) R. H. Holm, P. Kennepohl, E. I. Solomon, Chem. Rev. 1996, 96, 2239; c) J. W. Whittaker, Chem. Rev. 2003, 103, 2347; d) R. Banerjee, Chem. Rev.(Editorial); 2003, 103, 2081.

${ }^{94}$ a) R. H. Holm, E. I. Solomon, Chem. Rev. 2004, 104, 347; b) B. A. Jazdz ewski, W. B. Tolman, Coord. Chem. Rev. 2000, 200-202, 633; c) V. Mahadevan, R. J. M. Klein Gebbink, T. D. P. Stack, Curr. Opin. Chem. Biol. 2000, 4, 228. d) Y. Wang, J. L. Dubois, B. Hedman, K. O. Hodgson, T. D. P. Stack, Science 1998, 279, 537; e) F. Thomas, G. Gellon, I. GautierLuneau, E. Saint-Aman, J.-L. Pierre, Angew. Chem., Int. Ed. 2002, 41, 3047; f) Y. Wang, T. D. P. Stack, J. Am. Chem. Soc. 1996, 118, 13097. 
95 a) N. Ito, S. E. V. Phillips, C. Stevens, Z. B. Ogel, M. J. McPherson, J. N. Keen, K. D. S. Yadav, P. F. Knowles, Nature 1991, 350, 87; b) N. Ito, S. E. V. Phillips, K. D. S. Yadav, P. F. J. Knowles, Mol. Biol. 1994, 238, 799; c) R. M. Wachter, B. P. Branchaud, J. Am. Chem. Soc. 1996, 118, 2782; d) C. Wright, A. G. Sykes, J. Inorg. Biochem. 2001, 85, 237.

96 (a) A. Ghosh, T. Wondimagegn, E. Gonzalez, I. Halvorsen, D. Dolphin, T. Niem, R. H. Felton, I. Fujita, J. Am. Chem. Soc. 1975, 97, 5288; (b) D. Chang, T. Malinski, A. Ulman, K. M. Kadish, Inorg. Chem. 1984, 23, 817; (c) J. Seth, V. Palaniappan, D. F. Bocian, Inorg. Chem. 1995, 34, 2201; (d) M. W. Renner, J. Fajer, J. Biol. Inorg. Chem. 2001, 6, 823.

97 a) H. Steinhagen, G. Helmchen, Angew. Chem. Int. Ed. Engl. 1996, 35, 2339; b) E.K. Van den Beuken, B.L. Feringa, Tetrahedron 1998, 54, 12985; c) B. Bosnich, Inorg. Chem. 1999, 38, 2554 ; (d) C. Belle, J.-L. Pierre, Eur. J. Inorg. Chem. 2003, 4137.

98 a) P. Chaudhuri, M. Hess, J. Müller, K. Hildenbrand, E. Bill, T. Weyhermüller, K. Wieghardt, J. Am. Chem. Soc. 1999, 121, 9599; b) Y. Wang, J. L. DuBois, B. Hedman, K. O. Hodgson, T. D. P. Stack, Science 1998, 279, 537; c) E. Saint-Aman, S. Menage, J.-L. Pierre, E. Degrancq, G. Gellou, New J. Chem. 1998, 393; d) O. Rotthaus, O. Jarjayes, F. Thomas, C. Philouze, E. S. Aman, J.-L. Pierre, Dalton Trans. 2007, 889.

99 a) Y. Shimazaki, F. Tani, K. Fukui, Y. Naruta, O. Yamauchi, J. Am. Chem. Soc. 2003, 125, 10512; b) Y. Shimazaki, T. Yajima, F. Tani, S. Karasawa, K. Fukui, Y. Naruta, O. Yamauchi, J. Am. Chem. Soc. 2007, 129, 2559; c) O. Rotthaus, F. Thomas, O. Jarjayes, C. Philouze, E. Saint-Aman, J.-L. Pierre, Chem. Eur. J. 2006, 12, 6953; d) O. Rotthaus, O. Jarjayes, F. Thomas, C. Philouze, C. Perez Del Valle, E. Saint-Aman, J.-L. Pierre, Chem. Eur. J. 2006, 12, 2293; e) T. Storr, E. C. Wasinger, R. C. Pratt, T. D. P. Stack, Angew. Chem. Int. Ed. 2007, $46,5198$.

100 a) S.V. Kryatov, E.V. Rybak-Akimova, F. Meyer, H. Pritzkow, Eur. J. Inorg. Chem. 2003, 1581; b) B. Bauer-Siebenlist, F. Meyer, E. Farkas, D. Vidovic, J.A.C. Seijo, R. Herbst-Irmer, H. Pritzkow, Inorg. Chem. 2004, 43, 4189; c) B. Bauer-Siebenlist, F. Meyer, E. Farkas, D. Vidovic, S. Dechert, Chem. Eur. J. 2005, 11, 4349; d) B. Bauer-Siebenlist, S. Dechert, F. Meyer, Chem. Eur. J. 2005, 11, 5343. 
101 a) C. Vichard, T.A. Kaden, Inorg. Chim. Acta 2002, 337, 173; b) C. Vichard, T. A. Kaden, Inorg. Chim. Acta 2004, 357, 2285.

102 a) A. Prokofieva, S. Dechert, C. Große, G. M. Sheldrick, F. Meyer, Chem. Eur. J. 2009, 15, 4994; (b) A. Prokofieva, A. I. Prikhod'ko, S. Dechert, F. Meyer, Chem.Commun. 2008, 1005.

${ }^{103}$ a) T. G. Schenck, J. M. Downes, C. R. C. Milne, P. B. Mackenzie, H. Boucher, J. Whelan, B. Bosnich, Inorg. Chem. 1985, 24, 2334; b) J. C. Roeder, F. Meyer, H. Pritzkow, Organometallics 2001, 20, 811; c) T. Kamiusuki, H. Okawa, K. Inoue, N. Matsumoto, M. Kodera, S. Kida, J. Coord. Chem.1991, 23, 201.

104 (a) A. Prokofieva, A. I. Prikhod'ko, E. A. Enyedy, E. Farkas, W. Maringgele, S. Demeshko, S. Dechert, F. Meyer, Inorg. Chem. 2007, 46, 4298, (b) F. Meyer, A. Jacobi, L. Zsolnai, Chem. Ber./Recl. 1997, 130, 1441; (c) J. Teichgräber, G. Leibeling, S. Dechert, F. Meyer, Z. Anorg. Allg. Chem. 2005, 631, 2613.

105 a) A. Sokolowski, J. Müller, T. Weyhermüller, R. Schnepf, P. Hildebrandt, K. Hildenbrand, E. Bothe, K. Wieghardt, J. Am. Chem. Soc. 1997, 119, 8889; b) L. Benisvy, E. Bill, A. J. Blake, D. Collison, E. S. Davies, C. D. Garner, G. McArdle, E. J. L. McInnes, J. McMaster, S. H. K. Rossa, Claire Wilsona, Dalton Trans. 2006, 258.

106 a) P. A. Connick, K.A. Macor, Inorg. Chem. 1991, 30, 4654; b) T. J. Collins, T. R. Nichols, E. S. Uffelman, J. Am. Chem. Soc. 1991, 113, 4708; c) F. Azevedo, M.A. Carrondo, B. Castro, M. Convery, D. Domingues, C. Freire, M. T. Duarte, K. Nielsen, I. C. Santos, Inorg. Chim. Acta 1994, 219, 43; d) D. Pinho, P. Gomes, C. Freire, B. De Castro, Eur. J. Inorg. Chem. 2001, 1483; e) Z. Xiao, B. O. Patrick, D. Dolphin, Inorg. Chem. 2003, 42, 8125.

107 a) J. Müller, A. Kikuchi, E. Bill, T. Weyhermüller, P. Hildebrandt, L. Ould-Moussa, K. Wieghardt, Inorg. Chim. Acta 2000, 297, 265; b) Y. Shimazaki, S. Huth, S. Karasawa, S. Hirota, Y. Naruta, O. Yamauchi, Inorg. Chem. 2004, 43, 7816.

${ }^{108}$ G. M. Sheldrick, SHELXL-97, Program for Crystal Structure Renement, University of Göttingen, Göttingen, Germany, 1997. 
${ }^{109}$ G. M. Sheldrick, SHELXS-97, Program for Crystal Structure Solution, University of Göttingen, Göttingen, Germany, 1997.

${ }^{110}$ G. M. Sheldrick, Acta Cryst. 2008, A64, 112. 


\section{Appendix A: List of Free Ligands}<smiles>O=C(NNc1nc(NNC(=O)c2ccccn2)nc(NNC(=O)c2ccccn2)n1)c1ccccn1</smiles><smiles>[R]N(N)c1nc(N([R])N)nc(N(N)[18OH])n1</smiles>

1; $\mathrm{R}=\mathrm{CH}_{3}$

2; $\mathrm{R}=\left(\mathrm{CH}_{2}\right)_{2} \mathrm{OH}$

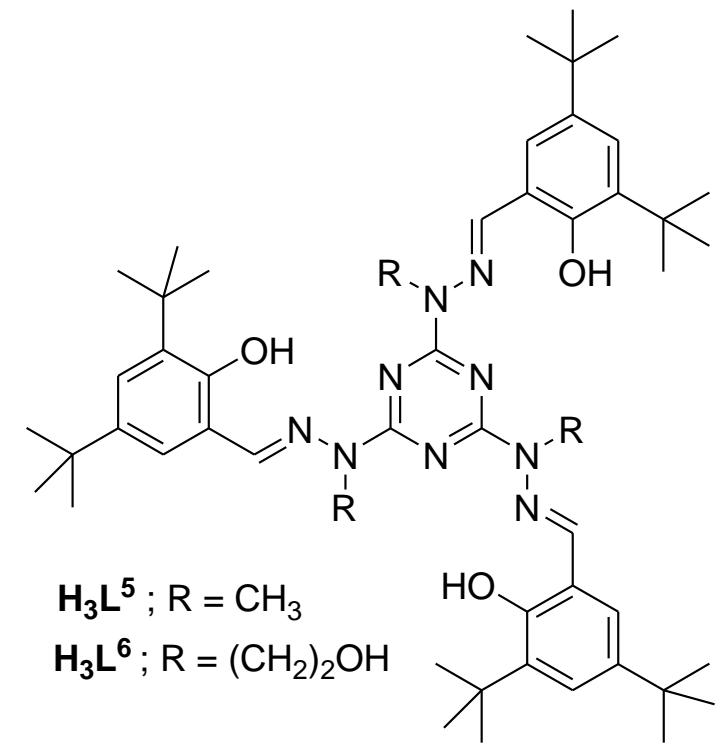<smiles>Oc1cccnc1-c1nc(-c2ncccc2O)nc(-c2ncccc2O)n1</smiles>

$\mathrm{H}_{3} \mathrm{~L}^{2}$

$\mathrm{H}_{3} \mathrm{~L}^{3} ; \mathrm{R}=\mathrm{CH}_{3}$

$\mathrm{H}_{3} \mathrm{~L}^{4} ; \mathrm{R}=\left(\mathrm{CH}_{2}\right)_{2} \mathrm{OH}$

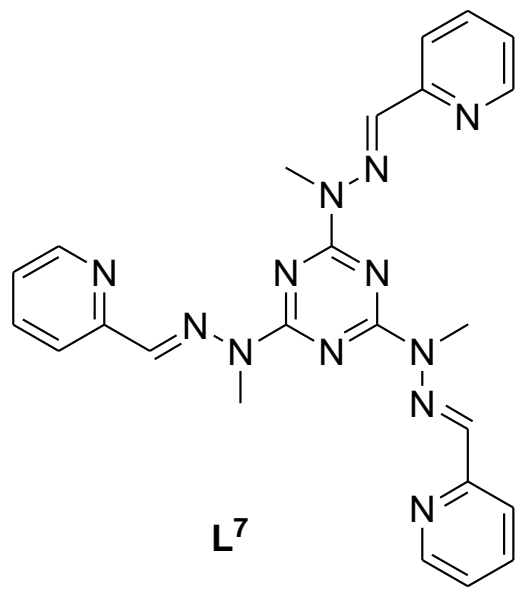


<smiles>Cc1cc(-c2ccccc2O)n[nH]1</smiles>

$H_{2} L^{8}$<smiles>OCCn1nc(-c2ccccc2O)cc1-c1ccccc1</smiles>

$H_{2} L^{10}$<smiles>OCCn1ccc(-c2ccccc2O)n1</smiles>

$\mathrm{H}_{2} \mathrm{~L}^{9}$

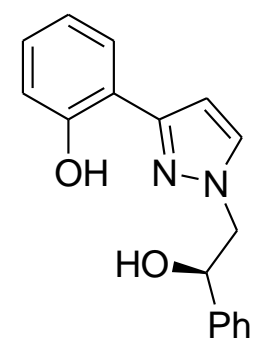

(R)- $\mathrm{H}_{2} \mathrm{~L}^{11}$

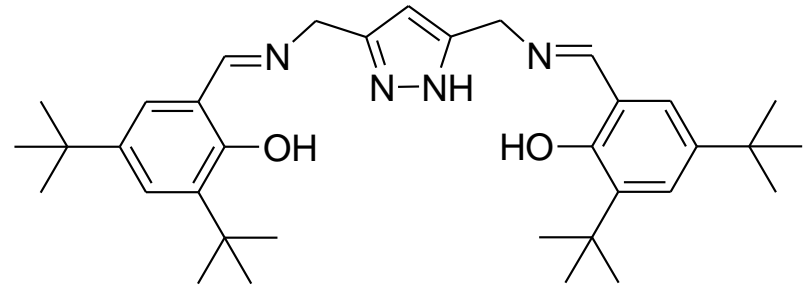

$\mathrm{H}_{3} \mathrm{~L}^{12}$ 


\section{Appendix B: List of crystalline compounds}

3. $\quad\left[\left\{\mathrm{L}^{5 \mathrm{a}} \mathrm{Cu}_{2}{ }_{2}\left(\mathrm{H}_{2} \mathrm{O}\right)_{2}(\mathrm{MeOH})_{2}\right\}\left\{\mathrm{L}^{5 \mathrm{a}} \mathrm{Cu}_{2}{ }_{2}(\mathrm{MeOH})_{4}\right\}\right] 2\left(\mathrm{NO}_{3}\right) \cdot \mathrm{xCH}_{3} \mathrm{OH} \cdot \mathrm{yH}_{2} \mathrm{O}$

4. $\quad\left[\mathrm{Mn}_{4}^{\mathrm{IIII}}\left(\mathrm{H}_{2} \mathrm{~L}^{6 \mathrm{a}}\right)_{4}\right] \cdot \mathrm{xCH}_{3} \mathrm{OH} \cdot \mathrm{yCH}_{3} \mathrm{CN}$

5. $\quad\left[\mathrm{Fe}^{\mathrm{III}}{ }_{4}\left(\mu_{2}-\mathrm{O}\right)\left(4 \mathrm{H}_{2} \mathrm{O}\right)\left(\mathrm{H}_{2} \mathrm{~L}^{6 \mathrm{a}}\right)_{2}\left(\mathrm{~L}^{\mathrm{t}-\mathrm{Bu}}{ }_{2}\right)_{2}\right]\left(\mathrm{NO}_{3}\right)_{2} \cdot 3 \mathrm{CH}_{3} \mathrm{OH} \cdot 6 \mathrm{CH}_{3} \mathrm{CN}$

6. $\left[\mathrm{Mn}^{\mathrm{II}}\left(\mathrm{L}^{7}\right)\left(\mathrm{NO}_{3}\right)\left(\mathrm{H}_{2} \mathrm{O}\right)\right]\left(\mathrm{NO}_{3}\right) \cdot \mathrm{xCH}_{3} \mathrm{OH}$

7. $\quad\left[\mathrm{Mn}_{2}{ }_{2}\left(\mathrm{~L}^{7}\right)_{2}\left(\mathrm{~N}_{3}\right)\left(\mathrm{NO}_{3}\right)\left(\mathrm{H}_{2} \mathrm{O}\right)\right]\left(\mathrm{NO}_{3}\right)_{2} \cdot \mathrm{yH}_{2} \mathrm{O}$

8. $\quad\left[\mathrm{Mn}^{\mathrm{II}}\left(\mathrm{L}^{7}\right)\left(\mathrm{N}_{3}\right)_{2}\right] \cdot \mathrm{C}_{2} \mathrm{H}_{8} \mathrm{O}_{2}$

9. $\quad\left[\mathrm{Mn}^{\mathrm{II}}\left(\mathrm{L}^{7}\right)\left(\mathrm{N}_{3}\right)\right]_{\mathrm{n}}\left(\mathrm{NO}_{3}\right)_{\mathrm{n}} \cdot \mathrm{nCH}_{3} \mathrm{OH}$

10. $\left[\mathrm{Mn}^{\mathrm{II}} \mathrm{L}^{7}(\mathrm{dca})\left(\mathrm{H}_{2} \mathrm{O}\right)\right]\left(\mathrm{NO}_{3}\right) \cdot \mathrm{xCH}_{3} \mathrm{OH} \cdot \mathrm{yH}_{2} \mathrm{O}$

11. $\left[\mathrm{Ni}^{\mathrm{II}}{ }_{4}(\mathrm{MeOH})_{4}\left(\mathrm{~L}^{9}\right)_{4}\right] \cdot \mathrm{H}_{2} \mathrm{O}$

12. $\left[\mathrm{Co}_{4}{ }_{4}(\mathrm{MeOH})_{3}\left(\mathrm{H}_{2} \mathrm{O}\right)\left(\mathrm{L}^{9}\right)_{4}\right] \cdot \mathrm{CH}_{2} \mathrm{Cl}_{2}$

13. $\quad\left[\mathrm{Fe}_{4}{ }_{4}(\mathrm{MeOH})_{4}\left(\mathrm{~L}^{9}\right)_{4}\right]$

14. $8 \mathrm{CH}_{2} \mathrm{Cl}_{2} \quad\left[\mathrm{Mn}_{2}{ }_{2} \mathrm{Ni}{ }_{3}{ }_{3}\left(\mathrm{~L}^{9}\right)_{4}\left(\mathrm{HL}^{9}\right)_{2} \mathrm{Cl}_{2}\left(\mathrm{H}_{2} \mathrm{O}\right)_{2}\right] \cdot 8 \mathrm{CH}_{2} \mathrm{Cl}_{2}$

14· $6 \mathrm{C}_{2} \mathrm{H}_{4} \mathrm{Cl}_{2} \quad\left[\mathrm{Mn}_{2}{ }_{2} \mathrm{Ni}^{\mathrm{II}}{ }_{3}\left(\mathrm{~L}^{9}\right)_{4}\left(\mathrm{HL}^{9}\right)_{2} \mathrm{Cl}_{2}\left(\mathrm{H}_{2} \mathrm{O}\right)_{2}\right] \cdot 6 \mathrm{C}_{2} \mathrm{H}_{4} \mathrm{Cl}_{2}$

15. $8 \mathrm{CH}_{2} \mathrm{Cl}_{2} \quad\left[\mathrm{Mn}_{2}{ }_{2} \mathrm{Ni}^{\mathrm{II}}{ }_{3}\left(\mathrm{~L}^{9}\right)_{4}\left(\mathrm{HL}^{9}\right)_{2} \mathrm{Br}_{2}\left(\mathrm{H}_{2} \mathrm{O}\right)_{2}\right] \cdot 8 \mathrm{CH}_{2} \mathrm{Cl}_{2}$

16. $\left[\mathrm{Mn}^{\mathrm{III}}{ }_{2} \mathrm{Mn}^{\mathrm{II}}\left(\mathrm{L}^{9}\right)_{2}(\mathrm{OAc})_{2}(\mathrm{OMe})_{2}(\mathrm{MeOH})_{2}\right] \cdot \mathrm{MeOH}$

17. $\left.\left[\mathrm{Mn}^{\mathrm{III}}{ }_{4} \mathrm{Mn}_{2}{ }_{2} \mathrm{~L}^{\mathrm{II}}\right)_{4} \mathrm{Cl}_{4}\left(\mathrm{H}_{2} \mathrm{O}\right)_{4}\left(\mu_{4}-\mathrm{O}\right)_{2}\right] \cdot 4 \mathrm{CH}_{3} \mathrm{CN}$ 
18.

$\left[\mathrm{NHEt}_{3}\right]\left[\left(\mathrm{L}^{9}\right)_{2} \mathrm{Mn}_{2}^{\mathrm{III}}\left\{\left(\mathrm{NO}_{3}\right)_{2} \mathrm{Gd}^{\mathrm{III}}\right\}_{2}\left(\mathrm{O}_{2} \mathrm{CPh}\right)_{3}\left(\mu_{4}-\mathrm{O}\right)\right] \cdot \mathrm{xCH}_{3} \mathrm{CN} \cdot \mathrm{yCH}_{2} \mathrm{Cl}_{2}$

19.

$\left.\left[\mathrm{NHEt}_{3}\right]\left[\left(\mathrm{L}^{9}\right)_{2} \mathrm{Mn}_{2}^{\mathrm{III}} 2\left(\mathrm{NO}_{3}\right)_{2} \mathrm{~Tb}^{\mathrm{III}}\right\}_{2}\left(\mathrm{O}_{2} \mathrm{CPh}\right)_{3}\left(\mu_{4}-\mathrm{O}\right)\right] \cdot \mathrm{xCH}_{3} \mathrm{CN} \cdot \mathrm{yCH}_{2} \mathrm{Cl}_{2}$

20.

$\left[\mathrm{NHEt}_{3}\right]\left[\left(\mathrm{L}^{9}\right)_{2} \mathrm{Mn}_{2}^{\mathrm{III}}{ }_{2}\left\{\left(\mathrm{NO}_{3}\right)_{2} \mathrm{Dy}{ }^{\mathrm{III}}\right\}_{2}\left(\mathrm{O}_{2} \mathrm{CPh}\right)_{3}\left(\mu_{4}-\mathrm{O}\right)\right] \cdot \mathrm{xCH}_{3} \mathrm{CN} \cdot \mathrm{yCH}_{2} \mathrm{Cl}_{2}$

21.

$\left[\mathrm{L}^{12} \mathrm{Cu}_{2}\left(\mu-\mathrm{MeOH}^{\cdots} \mathrm{OH}\right)\right] \cdot 2.5 \mathrm{MeOH}$

22.

$\left[\mathrm{L}^{12} \mathrm{Cu}_{2}(\mu-\mathrm{OAc})\right] \cdot 1.33 \mathrm{CHCl}_{3}$

23.

$\left[\mathrm{L}^{12} \mathrm{Ni}_{2}(\mu-\mathrm{OAc})\right] \cdot 1.33 \mathrm{CHCl}_{3}$

24.

$\left[\mathrm{Zn}_{4}\left(\mathrm{~L}^{12}\right)_{2}\left(\mathrm{H}_{2} \mathrm{~L}^{12}\right)_{2}\right]$ 


\title{
Appendix C: List of Chemical Terms
}

\author{
$\mathbf{H}_{3} \mathbf{L}^{\mathbf{1}} \quad$ 2,4,6-Tris( $N$-hydrazino-pyridyl-2-carboxylate)-1,3,5-triazine \\ $\mathbf{H}_{3} \mathbf{L}^{2} \quad$ 2,4,6-Tris(3-hydroxy-2-pyridyl)-1,3,5-triazine \\ $1 \quad 2,4,6$-Tris $(N$-methylhydrazino)-1,3,5-triazine \\ 2 2,4,6-Tris $\{N$-(2-hydroxyethylhydrazino) $\}$-1,3,5-triazine \\ $\mathbf{H}_{3} \mathbf{L}^{\mathbf{3}} \quad$ 2,4,6-Tris[ $\left(N\right.$-methyl- $N^{\prime}$-salicylaldimino)hydrazino]-1,3,5-triazine \\ $\mathbf{H}_{6} \mathbf{L}^{4} \quad$ 2,4,6-Tris[ $\left\{N\right.$-(2-hydroxyethyl)- $N^{\prime}$-(3,5-di-tert- butylsalicylaldimino) $\}$ \\ hydrazino]-1,3,5-triazine \\ $\mathbf{H}_{3} \mathbf{L}^{\mathbf{5}} \quad$ 2,4,6-Tris[ $\left\{N\right.$-methyl- $N^{\prime}$-(3,5-di-tert-butylsalicylaldimino) $\}$ hydrazino]- \\ $1,3,5$-triazine \\ $\mathbf{H}_{6} \mathbf{L}^{\mathbf{6}} \quad$ 2,4,6-Tris[ $\left\{N\right.$-(2-hydroxyethyl)- $N^{\prime}$-(3,5-di-tert- butylsalicylaldimino) $\}$ \\ hydrazino]-1,3,5-triazine \\ $\mathbf{L}^{7} \quad 2,4,6$-Tris $\left[\left\{N\right.\right.$-methyl- $N^{\prime}$-(pyridine-2-yl)methylidene $\}$ hydrazino $]-1,3,5$ - \\ triazine \\ $\mathbf{H}_{2} \mathbf{L}^{\mathbf{8}} \quad$ 3(5)-methyl-5(3)-(2-hydroxyphenyl)pyrazole pyrazole \\ $\mathbf{H}_{2} \mathbf{L}^{9} \quad$ 2-[3-(2-Hydroxyphenyl)-1H-pyrazol-1-yl] ethanol \\ $\mathbf{H}_{2} \mathbf{L}^{10} \quad$ 2-[\{3-(2-Hydroxyphenyl)-5-phenyl $\}$-1H-pyrazol-1-yl]ethanol \\ $\mathbf{R}-\mathbf{H}_{2} \mathbf{L}^{11} \quad$ R-2-[1-(2-Hydroxy-2-phenylethyl)-1H-pyrazol-3-yl]-phenol \\ $\mathbf{H}_{3} \mathbf{L}^{\mathbf{1 2}} \quad$ 3,5-Bis[(3,5-di-tert-butylsalicylaldimino)methyl]pyrazole
}




\section{Appendix D: Overview of Magnetic Parameters}

\begin{tabular}{|c|c|c|c|c|c|}
\hline compound & $g$ & $J_{1}, \mathrm{~cm}^{-1}$ & $J_{2}, \mathrm{~cm}^{-1}$ & $\begin{array}{l}|D|_{\text {ion }}, \\
\mathrm{cm}^{-1}\end{array}$ & $\begin{array}{l}\text { TIP } \\
10^{-6} \mathrm{~cm}^{3} \mathrm{~mol}^{-1}\end{array}$ \\
\hline 3 & 2.03 & -30 & & - & 1.7 \\
\hline 4 & 1.99 & -0.07 & & 0.3 & 0 \\
\hline 5 & 2.12 & -1.00 & \begin{tabular}{|l|}
-14.7 \\
\end{tabular} & - & 834.2 \\
\hline 6 & 1.96 & & & - & 248.9 \\
\hline 7 & 1.97 & -1.97 & & - & 472.3 \\
\hline 8 & 2.05 & & & - & 0 \\
\hline 9 & 2.02 & -1.23 & & & 0 \\
\hline 10 & 1.94 & & & - & 353.8 \\
\hline 11 & 2.22 & +6.86 & -4.86 & 10 & 227.3 \\
\hline 12 & 2.50 & +1.57 & -3.94 & - & 400.0 \\
\hline 13 & 2.19 & +7.04 & -4.90 & 7.5 & 1907.9 \\
\hline $\mathbf{1 4} \cdot 8 \mathrm{CH}_{2} \mathrm{Cl}_{2}$ & 2.1 & 12.6 & 8.4 & $-0.35^{\lfloor a\rfloor}$ & \\
\hline $\mathbf{1 5} \cdot 8 \mathrm{CH}_{2} \mathrm{Cl}_{2}$ & 2.1 & 29.4 & 12.6 & $-0.32^{\lfloor a\rfloor}$ & \\
\hline 16 & $2.16 \& 1.87$ & -2.63 & & 5.9 & 150 \\
\hline 17 & - & - & & - & - \\
\hline 18 & - & - & & - & - \\
\hline \multicolumn{6}{|l|}{19} \\
\hline \multicolumn{6}{|l|}{20} \\
\hline 21 & 2.1 & -27.53 & & - & 12.9 \\
\hline 22 & 2.1 & -36.88 & & - & 868.9 \\
\hline
\end{tabular}

[a] Easy-axis magentoanisotropy $\left(D_{\mathrm{mol}}\right)$. 


\section{Appendix E: Overview of Electrochemical Measurements ${ }^{[a]}$}

\begin{tabular}{|l|l|l|l|l|}
\hline compound & solvent & $E_{1 / 2}{ }^{1}[\mathrm{~V}]$ & $E_{1 / 2^{2}}[\mathrm{~V}]$ & $E_{\mathrm{p}, \text { ox }}^{3}[\mathrm{~V}]^{[\mathrm{c}]}$ \\
\hline $\mathbf{3}$ & $\mathrm{CH}_{3} \mathrm{CN}$ & -0.84 & $0.583,{ }^{[\mathrm{b}]} 0.637^{[\mathrm{b}]}$ & 1.19 \\
\hline $\mathbf{4}$ & THF & $-1.24^{[\mathrm{a}]}$ & $\begin{array}{l}0.10,^{[\mathrm{b}]} 0.187,,^{[\mathrm{b}]} \\
0.292^{[\mathrm{b}]} 0.366^{[\mathrm{b}]}\end{array}$ & 0.78 \\
\hline $\mathbf{1 2}$ & $\mathrm{CH}_{2} \mathrm{Cl}_{2}$ & & $0.14^{[\mathrm{c}]}$ & \\
\hline $\mathbf{1 3}$ & $\mathrm{CH}_{2} \mathrm{Cl}_{2}$ & & $0.546^{[\mathrm{c}]}$ & $0.778^{[\mathrm{c}]}$ \\
\hline $\mathbf{2 2}$ & $\mathrm{CH}_{2} \mathrm{Cl}_{2}$ & & $0.648,0.787$ & \\
\hline $\mathbf{2 3}$ & $\mathrm{CH}_{2} \mathrm{Cl}_{2}$ & & $0.570,0.650$ & \\
\hline $\mathbf{2 4}$ & $\mathrm{CH}_{2} \mathrm{Cl}_{2}$ & & $0.572,0.682$ & 0.900 \\
\hline
\end{tabular}

[a] Recorded at RT in presence of $(0.1 \mathrm{M})\left[(n-\mathrm{Bu})_{4} \mathrm{~N}\right]\left(\mathrm{PF}_{6}\right)$, and $\mathrm{Fc} / \mathrm{Fc}^{+}$is the reference electrode. [b] Data obtained from digital simulation of experimental CVs or from deconvolution of the experimental SWVs, respectively. [c] Peak potential for an irreversible redox process. [d] Quasi-reversible two electron redox process.

\section{Appendix F: Overview of Mössbauer Measurements}

\begin{tabular}{|l|l|l|l|}
\hline Compound & $\delta\left(\mathrm{nm} \mathrm{s}^{-1}\right)$ & $\Delta E_{\mathrm{Q}}\left(\mathrm{nm} \mathrm{s}^{-1}\right)$ & $\Gamma_{1 / 2}\left[\mathrm{~mm} \mathrm{~s}^{-1}\right]$ \\
\hline $\mathbf{5}^{[\mathrm{a}]}$ & 0.54 & 1.08 & 0.36 \\
\hline $\mathbf{7}^{[\mathrm{b}]}$ & 1.21 & 2.73 & 0.36 \\
\hline
\end{tabular}

[a] Recorded at 7 K. [b] Recorded at $80 \mathrm{~K}$. 


\begin{tabular}{ll} 
Abbreviation \\
FT & Fourier-transform \\
$H$ & Magnetic field \\
$H \mathrm{c}$ & Coercive field \\
$\mathrm{IR}$ & Infra red \\
$M$ & Magnetization \\
$\mathrm{NMR}$ & Nuclear magnetic resonance \\
$S$ & Spin state \\
$\mathrm{SMM}$ & Single-molecule magnet \\
$\mathrm{SQUID}$ & Superconducting Quantum Interference Devices \\
$T$ & Temperature \\
$T_{\mathrm{B}}$ & Blocking temperature \\
$T_{\mathrm{c}}$ & Curie temperature \\
$T_{\mathrm{N}}$ & Néel temperature \\
ZFS & Zero-field splitting \\
$\Delta E_{\mathrm{Q}}$ & Quadripole splitting \\
$\delta$ & Isomer shift (Mössbauer), Chemical shift (NMR) \\
$\varepsilon$ & Extinction coefficient \\
$\Gamma$ & Line widh parameter \\
$\mathrm{m}$ & Multiplet (NMR), Medium (IR) \\
$\mathrm{s}$ & Singlet (NMR), Strong (IR) \\
$S$ & Spin ground state \\
\hline & weak (IR) \\
\hline w &
\end{tabular}




\section{List of Scientific Contributions}

\section{Publications}

1. M. K. Ghorai, K. Das, A. Kumar, A. Das "A convenient synthetic route to 2-aryl- $N$ tosylazetidines and their $\mathrm{ZnX}_{2}(\mathrm{X}=\mathrm{I}$, OTf) mediated regioselective nucleophilic ring opening reactions: synthesis of $\gamma$-iodoamines and tetrahydropyrimidines" Tetrahedron Lett . 2006, 47, 5393.

2. A. Das, M. Linseis, S. Demeshko, S. Dechert, R. F. Winter, F. Meyer, “Triazine-Based Compartmental Schiff-Base Ligands and their Binuclear $\mathrm{Cu}(\mathrm{II})$ and Tetranuclear $\mathrm{Mn}(\mathrm{III})$ Complexes: Syntheses, Structures, Magnetic Properties and Spectro-Electrochemistry' in preparation for Inorg. Chem.

3. A. Das, K. Gieb, Y. Krupskaya, S. Demeshko, S. Dechert, R. Klingeler,V. Kataev, B. Büchner, P. Müller, F. Meyer, “A New Family of 1D Exchange Biased Heterometal Single Molecule Magnets: Observation of Quantum Tunneling Steps in the Hysteresis Loops of Linear $\left\{\mathrm{Mn}_{2} \mathrm{Ni}_{3}\right\}$ Clusters"' in preparation for J. Am. Chem. Soc.

4. A. Das, S. Demeshko, S. Dechert, V. Kataev, F. Meyer, "Pyrazole Based Homometallic Complexes: $\mathrm{M}_{4} \mathrm{O}_{4}\left(\mathrm{M}=\mathrm{Ni}^{\mathrm{II}}, \mathrm{Co}^{\mathrm{II}}, \mathrm{Fe}^{\mathrm{II}}\right)$ Cubes with High-Spin Ground States"' in preparation for Inorg. Chem.

5. A. Das, M. Pichlmaier, S. Demeshko, S. Dechert, R. F. Winter, F. Meyer, "New Binucleating Pyrazolate Ligands with Phenol Side arms- Potential Scaffolds for Bimetallic Multiradical Chemistry', in preparation for Chem. Eur. J.

6. A. Das, K. Gieb, S. Demeshko, S. Dechert, P. Müller, F. Meyer, "A New Family of Heterometallic [ $\left.\mathrm{Mn}_{2} \mathrm{Ln}_{2}\right]$ Single Molecule Magnets'' in preparation for Inorg. Chem.

7. A. Das, S. Demeshko, S. Dechert, F. Meyer, “Triazine Based Mononuclear, Dinuclear, and 1-D Chain Complexes of Manganese (II) with Some Auxiliary Ligands: Synthesis, Structures, and Magnetic Properties', in preparation for Eur. J. Inorg. Chem.

8. A. Das, K. Gieb, S. Demeshko, S. Dechert, P. Müller, F. Meyer, "High Nuclearity Aggregate of Mixed Valent Manganese Clusters" in preparation for Inorg. Chem. 


\section{Presentations at International Conferences and Workshops}

1. A. Das, S. Demeshko, S. Dechert, F. Meyer, "New binucleating pyrazolate ligands with phenol side arms- potential scaffolds for bimetallic multiradical chemistry" Göttinger Chemieforum, Göttingen, Germany, July 2008.

2. A. Das, S. Demeshko, S. Dechert, K. Gieb, P. Müller, F. Meyer "Homo- and Heterometallic Complexes of a Pyrazolate based $\{\mathrm{ONO}\}$ ligand: $\mathrm{M}_{4} \mathrm{O}_{4}$ Cubes with High-Spin Ground State and a New $\mathrm{Mn}^{\mathrm{III}} \mathrm{Ni}^{\mathrm{II}}{ }_{3} \mathrm{Mn}^{\mathrm{III}}$ Single Molecule Magnet', Colloquium of SPP 1137, Bad Dürkheim, Germany, February 2009.

3. A. Das, S. Demeshko, S. Dechert, K. Gieb, P. Müller, F. Meyer "Homo- and Heterometallic Complexes of a Pyrazolate based $\{\mathrm{ONO}\}$ ligand: $\mathrm{M}_{4} \mathrm{O}_{4}$ Cubes with High-Spin Ground State and a New $\mathrm{Mn}^{\mathrm{III}} \mathrm{Ni}^{\mathrm{II}}{ }_{3} \mathrm{Mn}^{\mathrm{III}}$ Single Molecule Magnet" Göttinger Chemieforum, Göttingen, Germany, July 2009.

4. A. Das, K. Gieb, Y. Krupskaya, S. Demeshko, S. Dechert, V. Kataev, P. Müller, F. Meyer, “A New Family of Pentanuclear Single Molecule Magnets: Observation of Quantum Tunneling Steps in the Hysteresis Loops of $\left\{\mathrm{Mn}^{\mathrm{III}} \mathrm{Ni}_{3}{ }_{3} \mathrm{Mn}^{\mathrm{III}}\right\}$ Clusters"' European Conference on Molecular Magnetism ECMM, Wroclaw, Poland, October 2009. 


\section{Acknowledgments}

The research presented in this thesis would not have been possible without the generous help and support of many people. In this section I would like to thank and acknowledge all of them.

Firstly, I would like to thank my advisor Prof. Dr. Franc Meyer, for his intellectual and professional guidance, for the opportunity to work in an exciting research group, for involving me in the preparation of many manuscripts, and especially for allowing me a large degree of independence and creative freedom to explore a wide range of synthetic aspirations.

Furthermore, I would like to address my sincere thanks to all colleagues and external collabor ators that added a great deal to the completion of this thesis. I am truly grateful to Prof. Dr. Paul Müller and Klaus Gieb for their sincere help in low temperature SQUID measurement and analyses, and I would like to thank Prof. Dr. Rainer Winter, Markus Pichlmaier, and Michael Linseis for electrochemistry, and spectroelcetrochemistry measurement. Many thanks to Dr. Vladislav Kataev, Rüdiger Klingeler, and Bernd Büchner for their help in HF-EPR measuremnts. For the X-ray analyses, I would like to acknowledge Dr. Sebastian Dechert who was very patient and showed endurance with all my "unexpected" structures also thanks for the resonance Raman measurements. I am especially thankful to Dr. Serhiy Demeshko for Mössbauer, and magnetic measurements. I am also grateful to him for his help in learning SQUID measurements, and analyses. Without his sincere help, this thesis would not be in its present form. Special thanks to Dr. Andrea Stückl for her enormous help and EPRmeasurements. I woul like to thank Jörg Teichgräber for cyclic voltammetry measurement at our institiute. I would like to thank Dr. Holm Frauendorf for performing all HRMS experiments and for his instructions regarding the use of the ESI spectrometers. I want to thank my labmate Dr. Daniel Olsson, Dr. Serhiy Demeshko, Iris Klawitter and Thomas Bayer for the excellent laboratory atmosphere (including useful discussions). Thanks to my other groupmates, Dr. Joachim Ballmann, Dr. Akhilesh Kumar Singh, Boris Burger, Michael Fuchs, Benjamin Schneider, Simone Wöckel, Dr. Joanna Galezowska, and friends Dr. Anukul Jana, Sakya S. Sen, Sarish P. Sankaranarayana, Alexander Döring, Prinson P. Samuel, Vimal Nair for valuable discussion and providing a friendly environment throughout my stay in Göttingen. The whole working group will remain in my heart.

I would also like to acknowledge all employees of the analytical laboratory, of the NMR and MS facilities, of the library, of the electrical and machine crafts shop and of the glassblowing crafts shop (especially Hans Joachim Schlette). Deep thanks furthermore to all house stewards 
and the cleaning service for maintaining the laboratory, to Matthias Hesse, Jörg Teichgräber and Andreas Schwarz for the distribution of chemicals and laboratory equipment, to Petra Unger and Dr. Andrea Stückl for handling all administrational issues and to Dr. Sebastian Dechert for assistance in all kinds of software problems.

A financial support from the DFG (Priority Program 1137 "Molecular Magnetism" and SFB 602) is gratefully acknowledged.

Last, but not least my deepest gratitude goes to my mother, brother, maternal uncles, grand father, and grand mother for their support during all these years. It is not easy to express in words, but I will always be there for you too. 


\section{Lebenslauf}

$\begin{array}{ll}\text { Name } & \text { Animesh Das } \\ \text { Geboren } & 29^{\text {th }} \text { May } 1983 \text { in Bakcha }\end{array}$

Staatsangehörigkeit Indisch

Schulbesuch

1988 - 1997 Primary School (Class I-IX)

$1997-1998 \quad$ Secondary School Examinations (Class X)

Studium

$1998-2000 \quad$ Higher Secondary School Examinations (Class XII)

$2000-2004 \quad$ B. Sc. (Hons.) in Chemie, Calcutta University, Indien

2004 - 2006 M. Sc. in Chemie, Indian Institute of Technology Kanpur, Indien

Promotion

Seit 2006 Promotion im Institut für Anorganische Chemie Göttingen unter der Leitung von Prof. Dr. Franc Meyer

Ph.D. Thesis "New Pyrazole- and Triazine-Based Ligands as Scaffolds for Oligonuclear Complexes: From Multiredox Stability to Hetereonuclear Single Molecule Magnets" 
